\title{
Features of Evolved Adaptive Responses (FEAR): an evolutionary-developmental model of psychological trauma and common mental illnesses
}

\author{
Dihan Wijewickrama
}

\begin{abstract}
The global burden of mental illness is massive and escalating. Depression alone has become the leading cause of disability worldwide. Current evidence-based treatments for the common forms of mental illness including depression, addiction, anxiety, psychosis and psychological trauma, have low effectiveness measured at the population level. The theoretical models upon which current treatments are based presume brokenness (meaning disease, dysfunction, disorder or dysregulation) somewhere in the human system and treatment seeks to target and correct or compensate for what is presumed to be broken. Yet, from autopsies to molecular and genetic studies, decades of biomedical research involving billions of dollars of funding have failed to validate a biological cause for any of the common forms of mental illness. Furthermore, the biomedical evidence-base that policymakers, funders, clinicians and the public rely upon for critical medical and public health decisionmaking has been shown to be systematically compromised and there is evidence both of skewing of the evidence-base to promote treatments such as Cognitive Behavioural Therapy and of widespread harm caused by prescribed psychotropic medications. Fixated on the individual and their presumed brokenness, current interventions and policy foci largely fail to address the validated social determinants of mental illness. Consequently, new approaches involving new models and new interventions are required to address the escalating burden of mental illness. A broad corpus of evidence, amassing across research disciplines, is presaging an ontological shift from a pathological to an evolutionary-developmental modelling of mental illness - opening pathways for a radical differentiation in recovery approaches, policy and cultural framing. This monograph surveys the cross-disciplinary evidence-base and presents an evolutionary-developmental model of trauma that may have broad applicability to other common forms of mental illness. In contrast to the putative disease-disorder-dysfunction-dysregulation model of mental illness, the evolutionarydevelopmental model describes common psychological conditions not as forms of biological failure but as forms of biological function - the psychological features of biological adaptations that have been conserved in evolution by providing survival advantages to the species even whilst sacrificing individual wellbeing. The model helps to explain the underlying biological mechanisms that connect the social environment to the development of mental illness. The permeability and plasticity of the neurophysiological and epigenetic regulatory systems involved in coordinating the evolved adaptive responses that underly common psychological conditions, present new foci for interventions to initiate and support recovery, healing and development. ${ }^{\dagger}$
\end{abstract}

\footnotetext{
† Many of us know people (or are ourselves the people) who have received significant benefit or indeed whose lives have been saved by biomedical healthcare. Advances in medicine and the commitment in healthcare to research and innovation make an undeniably vital contribution to human progress. Nevertheless, seeing the trees around us does not mean that we accurately perceive the state of the forest. The health of the population is the forest and is revealed through historical evidence and epidemiological data, which are classically impersonal and statistical. The story that's constructed through these sources differs considerably from orthodox narratives which model the historical improvement of public health as a function of biomedical healthcare. The intention for this monograph is not to disparage modern healthcare, but rather to use the tools of systemic inquiry to describe crucial limitations of the current orthodox biomedical and psychological approaches to mental illness - to establish why change is needed - and to pave the way for emerging scientific ideas, perspectives and models of the nature and treatment of common mental disorders.
} 


\section{Table of Contents}

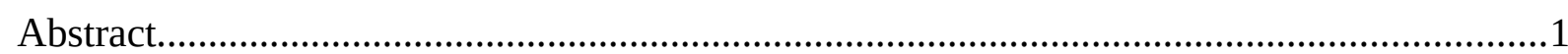

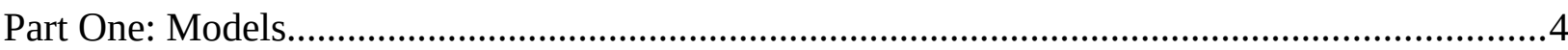

Models behaving badly..................................................................................................

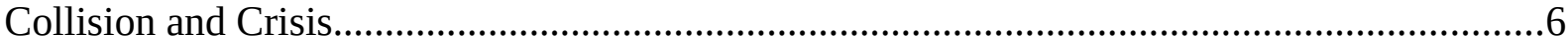

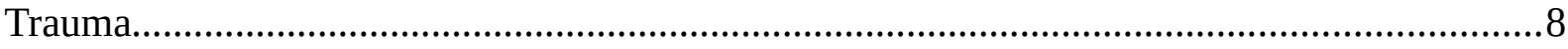

Part Two: Brokenness

Time doesn't heal all wounds...........................................................................................12

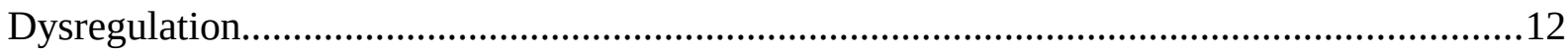

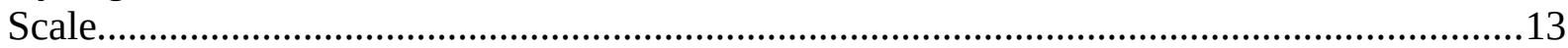

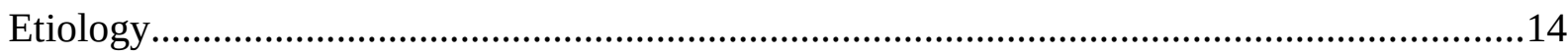

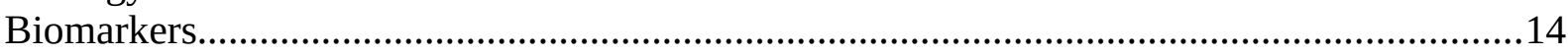

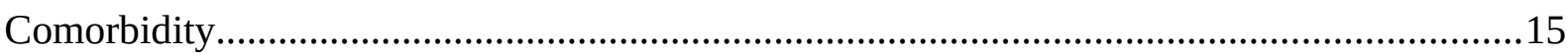

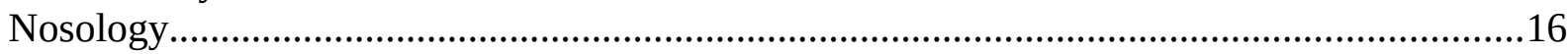

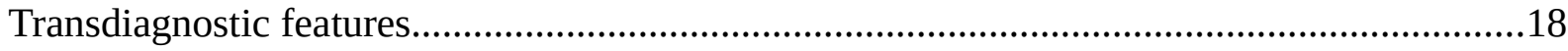

Treatment Effectiveness......................................................................................................20

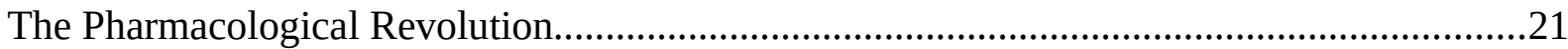

Insulin vs Alcohol Models of Psychoactive Drug Action..........................................................22

Doctoring the Science and Manufacturing Ignorance................................................................23

Psychotherapies and Cognitive Behavioural Therapy..............................................................27

Part Three: Complexity............................................................................................................32

Wicked Problems.......................................................................................................

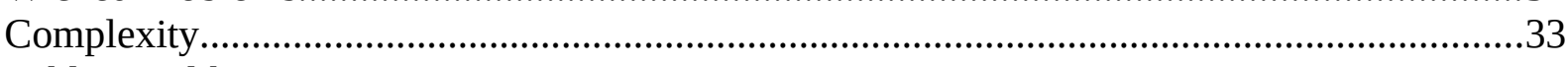

Public Health..................................................................................................................

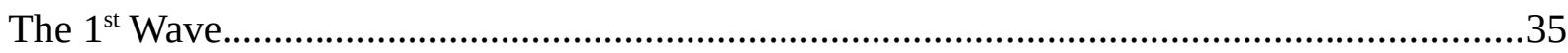

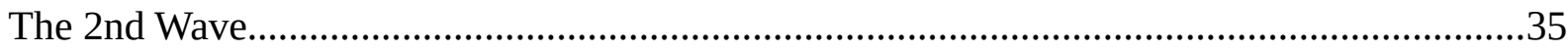

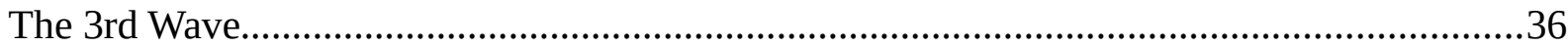

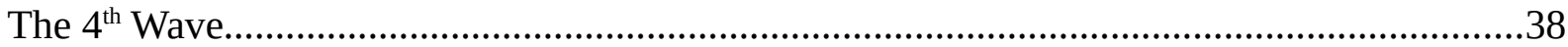

The Era of Individual Risk.............................................................................................39

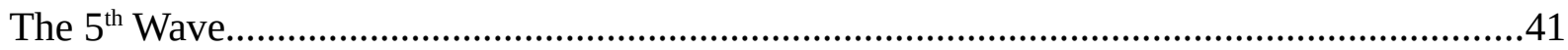

The Environmental Carriers of Disease............................................................................42

The Contribution of Medicine to Public Health...........................................................................43

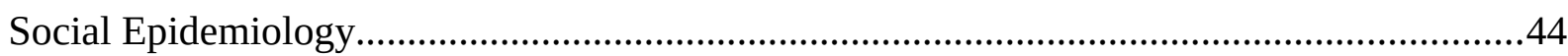

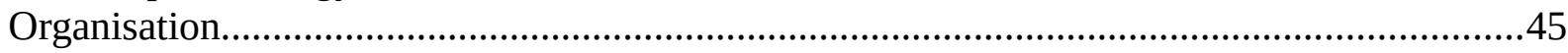

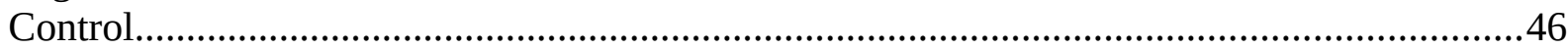

Community-led Public Health............................................................................................48

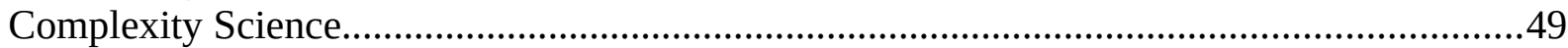

Investigating Health Disparities........................................................................................5

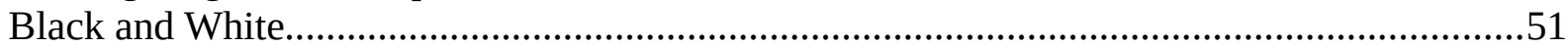

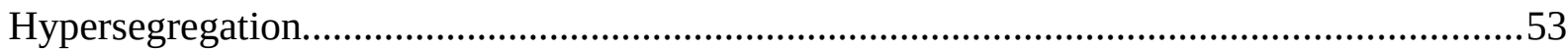

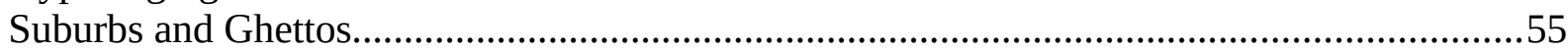

The Social Engineering of Inequality..................................................................................57

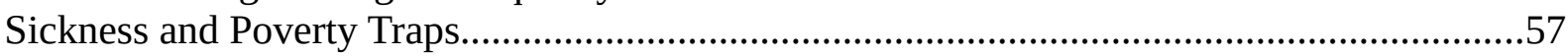

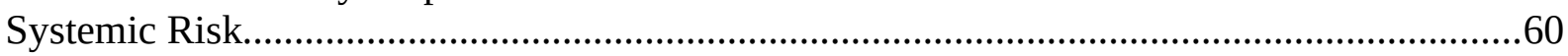

Racism as a Systemic Risk Factor....................................................................................60

How Systemic Risks Become Biological Processes..................................................................62

Catastrophising Teen Pregnancy.........................................................................................63

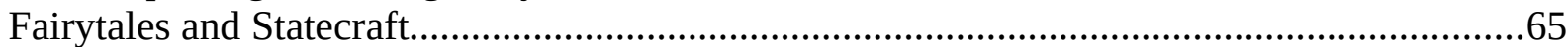

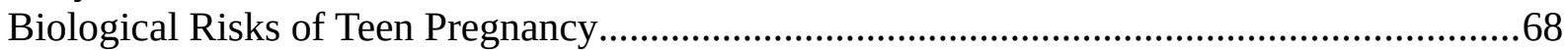

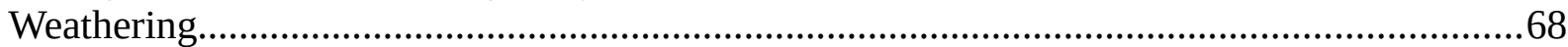

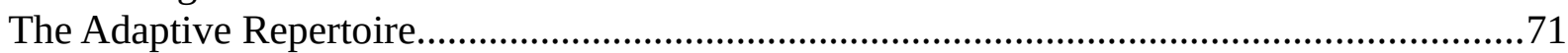




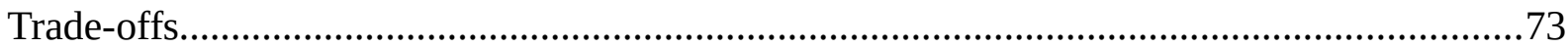

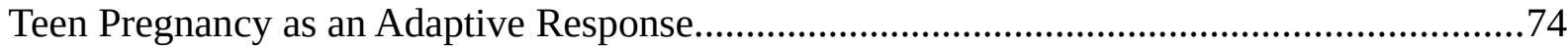

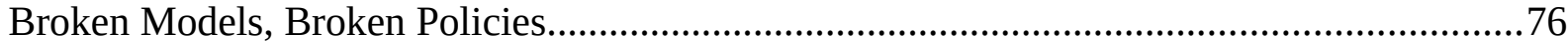

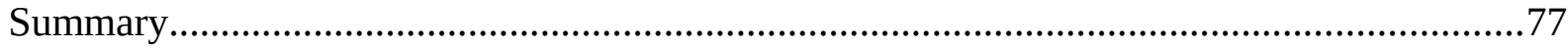

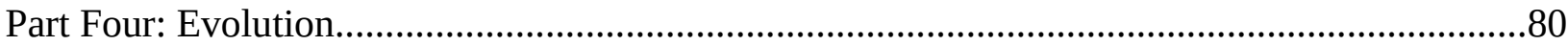

Evolved Mechanisms underlying Common Psychological Conditions...................................80

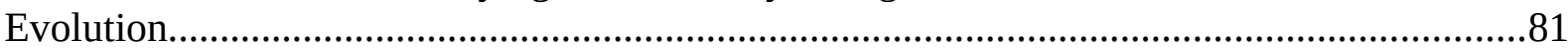

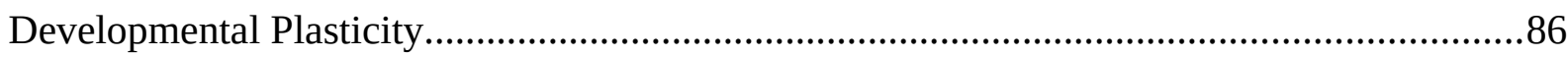

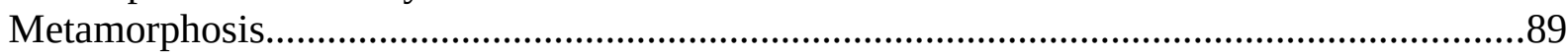

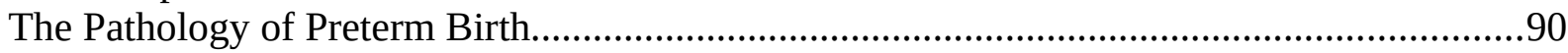

Idiopathic Preterm Birth as Evolved Adaptive Response......................................................93

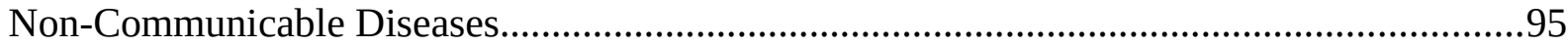

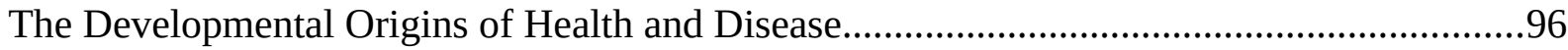

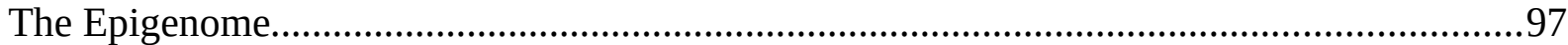

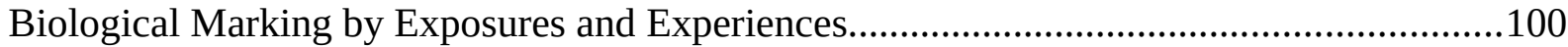

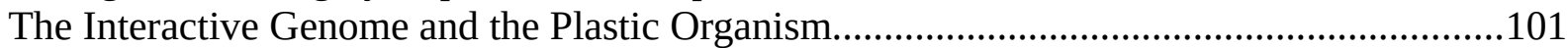

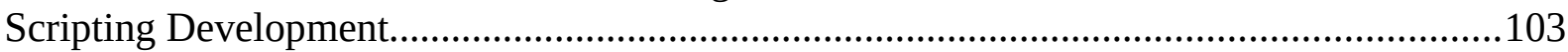

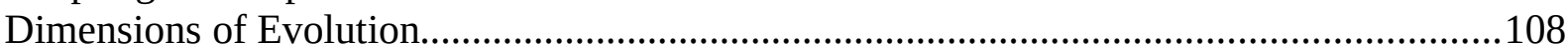

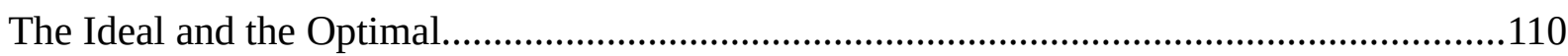

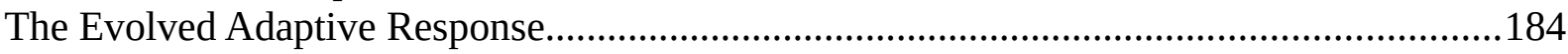




\section{Part One: Models}

Australians are seeing their doctor to discuss mental health ailments more than any other issue, according to a new [2018] report by the Royal Australian College of General Practitioners...A survey of more than 1,500 GPs ranked psychological ailments as the most common cause for a patient visit (62\%) respiratory conditions (45\%), musculoskeletal issues (43\%) and endocrine and metabolic problems (36\%). Half of the GPs surveyed said mental health was the issue causing them the most concern for the future, which ranked ahead of obesity (45\%), diabetes (18\%) aged care and the ageing population (14\%), drug addiction (12\%) and chronic pain and palliative care (8\%). The report said 87.8\% of Australians visited their GP at least once each year, while the more disadvantaged a patient was in socioeconomic terms, the more often they were likely to see a doctor. ${ }^{1,2}$

\section{Models behaving badly}

Mental and substance use disorders are now the leading cause of disability worldwide. This documented global burden of disease associated with mental disorders is compounded by the widening "mental health treatment gap" where, worldwide, more than $70 \%$ of persons who need mental health services lack access to care. ${ }^{3}$

Australia is one of the world's wealthiest nations and has comparatively high standards of healthcare ${ }^{4}$. And yet, in Australia, the need for comprehensive mental healthcare reform is both urgent and extensively documented ${ }^{5}$. There is broad and persistent demand for meaningful and systemic change - meaning new understandings, new strategies and new tools to address seemingly pervasive psychological maladies and to promote wellbeing 5 . Furthermore, the call for wholeof-system change is occurring in a global context of unprecedented socioeconomic disruption and institutional crises ${ }^{6,7}$. How can substantive and transformative change happen in a complex sociotechnical system such as the healthcare system? An historical case-study, from outside the field of healthcare, reveals how sociotechnical systems are conserved and constrained by realpolitik (pragmatism over idealism) and yet can be compelled to change through the escalation of environmental feedbacks signalling a crisis in how we imagine and model the world.

In northern Europe in the late 1700's, elements within the German political elite were beginning to complain vociferously that the poor were pillaging the forests. At the time, old-growth forests were a central pillar of state economies in central Europe ${ }^{8}$. The German elite argued that uncontrolled use of local forests by the peasantry was precipitating a crisis of critical resources of timber and fuel. To forestall the imminent

ـ This figure increased to 65\% in the General Practice: Health of the Nation 2019 report, while other leading concerns such as musculoskeletal, respiratory and endocrine and metabolic conditions declined. 
crisis, elite scholars introduced the idea of 'sustainability' as a principle of simultaneously exploiting and conserving natural resources to feed the growing appetites of the emerging industrial state and called for the creation of a state-based 'Scientific Forestry' to manage this nation building task'

However the supposed crisis was likely an exercise in alarmism; modern historians have been unable to identify any large-scale resource crisis in Germany between 1750-1850, and instead point to fluctuations in the supply of timber and fuel as periodic occurrences driven by market and political forces ${ }^{9}$.

Thus Scientific Forestry and the notion of sustainability originated and co-developed as instruments serving the political and economic needs of the early modern European states of Prussia and Saxony and have gone on to become the basis of intensive forest (plantation) management practices in France, England, the United States and throughout the European-colonised world for the last 200 years ${ }^{10,11}$.

The first step of the new Scientific Forestry was calculation; measuring the inventory, growth and yield of the forest according to the demands of commercial exploitation. A complicated calculus was produced to establish the volume of saleable wood from a 'standardised tree' (normalbaum) from data about size, age and conditions for normal growth and maturation.

The next step was to impose order over chaotic nature. Through precise seeding, planting, pruning, felling and clearing - the forest was repurposed for commercial production; remodelled according to a managerial ideal and redesigned with geometric exactitude. Accordingly, the understory of the forests was cleared, the diversity of chaotic old-growth was reduced often to a single preferred species (monoculture) and new plantings were made in straight orderly rows across large tracts of land.

The principles of Scientific Forestry were applied rigorously to most large forests in Germany throughout much of the nineteenth century. The project was hugely successful - initial commercial profits were stunning. The productivity of the new forests reversed a declining domestic wood supply and raised commercial returns from the forests. Subsequently, the German model of intensive commercial forestry became the world-standard - replicated in Europe, the US and in the British Colonies of India and Burma ${ }^{10,12}$.

Because of crop species that take about 80 years to mature, the unintended consequences of Scientific Forestry's 'sustainable management' practices took about a century to become obvious. The first crop of monocultured trees had grown so well because they were using up the long-accumulated 'soil-capital' of the diverse oldgrowth forest that they had replaced. Once this store of soil-wealth was depleted it was not replaced because the complex ecological cycle that created it had been 
destroyed $^{13}$. Production losses of $20-30 \%$ were observed in subsequent crops with steep declines in tree growth, yield and revenue. The wholesale clearing of the underbrush disrupted the food-webs of fungi, insects, mammals and birds critical for soil fertility. Monocultured forest plantations are vulnerable to storm felling and also produce epidemics of pests specialised to exploit the dominant species. Large outlays of fertilisers, insecticides and pesticides are generally required to combat escalating losses. A new word entered the German vocabulary to describe the extreme environmental consequences of Scientific Forestry - 'waldsterben' - meaning the death of the forest. The destruction of forest ecosystems under Scientific Management, drove German foresters to become pioneers of forest restoration and rehabilitation - for example by reintroducing bird and insect species into the environment and reconstituting their habitats. ${ }^{10,11}$

\section{Collision and Crisis}

we seldom really know the things we think we know

\section{Mark Twain ${ }^{14}$}

Scientific Forestry can be described as a model of reality. A model is a collection or set of ways for thinking about and believing in how the world (or some part of it) works ${ }^{15}$. Models serve to simplify reality, enable us to make sense of a complex world and to organise human activity at scale. Models consist of 'working ideas' and assumptions about reality that over time become axiomatic - part of 'received wisdom' or dogma. Generating models is also a core activity in science - where physical, conceptual and mathematical representations (abstractions) are used to explain and predict real-world phenomena such as climate models used for weather forecasting or wave-particle models for describing quantum phenomena ${ }^{16}$.

Models become more implicit and hypnotic as they become more pervasive and culturally normalised. Shared models become a basis for normative group behaviour, for example 'Clinical Guidelines' promoted as based on 'best evidence' or 'expert consensus' together with forces of professional regulation, compliance and auditing imposed on healthcare practitioners in western developed nations including Australia, which exert subtle and unsubtle pressures to conform and accept uncritically what may actually be untested axioms - simply in order to go about everyday professional life - and conduct business as usual ${ }^{17}$. Our models of the world shape what we pay attention to and what we ignore, what we perceive and how we act ${ }^{18}$. Thus models drive real impacts and consequences in the world.

Words and their definitions are the tip of the spear of models of reality, or put another way - words carry payloads of models and worldviews that deliver material impacts. A model of nation-building and commercial exploitation defines the forest as a natural resource - to be bureaucratically controlled and managed for the sustained 
benefit of the state and its ambitions. In contrast, a model of planetary ecology defines a forest differently - as a critically poised self-organising adaptive system and information-sharing network ${ }^{19}$, embedded in a web of coregulatory relationships (complex coupled cycles) with other systems in the world including the oceans and the atmosphere. In this systems model, forests can be depicted as vital components of a web of life - including being the supplier of critical 'ecosystem services' that human life depends on such as purification of water and air, carbon sequestration and climate regulation, predators that regulate pest species, pollination of plants and crop species, nutrient recycling and waste decomposition ${ }^{20}$. A recent World Health Organization report identified mechanisms connecting ecosystem biodiversity to human health, including:

Reduced contact of people with the natural environment and biodiversity, and biodiversity loss in the wider environment, leads to reduced diversity in the human microbiota [the massive bacterial colonisation of the human body which is now understood to be vital for human health and development], which itself can lead to immune dysfunction and disease. Considering microbial diversity as an ecosystem service provider may contribute to bridging the chasm between ecology and medicine/immunology, by considering microbial diversity in public health and conservation strategies aimed at maximising services obtained from ecosystems. $^{21}$

The 'wildness' or chaos of old-growth forests represents a complex entwined biodiverse system that supports and coevolves with planetary ecosystems and human life - and thus, commercial versus ecological models of the forest drive very different relationships and outcomes between people and environment.

The dying forests of nineteenth century Germany marked the point of collision between a model of the forest as an inert natural resource that could be appropriated, refashioned and commercialised 'sustainably' with the reality of the forest as a complex self-organising living system, governed by its own intrinsic dynamics and with a unique trajectory of development and evolution ${ }^{22}$. The failure of an entrusted model to predict and be congruent with salient features of the environment can compel a search for a new model and consequently, induce constructive change. But the failure of a dominant cultural model is non-trivial - producing a crisis of legitimacy, because such a model is not merely a theory or set of assumptions to be empirically validated or invalidated but a representation of reality (a worldview) that serves to legitimate a set of relationships between people and world - and if such a model is shown to be inaccurate or false, then not just ways of thinking but ways of being are threatened with collapse. The disruption and fall of cultural models can provoke an existential crisis.

In this paper, I contend that such an existential crisis is currently unfolding in the domain of mental health - as a dominant model which positions psychological trauma and common mental illnesses as forms of biological breakdown or failure (the 
disease-disorder-dysfunction-dysregulation model of mental illness) collides with a growing body of radically divergent evidence emerging from a broad array of fields including neurophysiology, evolutionary and developmental biology, social genomics and epidemiology.

Accumulating evidence ${ }^{23,24}$ from these diverse fields is reframing the scientific understanding of psychological trauma and other common forms of mental illness, from forms of biological failure to forms of biological function - features of evolved adaptive responses to developmental and environmental conditions. According to an emerging evolutionary-developmental paradigm - the common forms of mental illness including depression, anxiety, addiction, psychological trauma, bipolar disorder and psychosis ${ }^{25-27}$ represent the psychological features of underlying biological adaptations to the environment which may be initiated by developmental or life-course events which may span generations and which are broadly conserved in evolution because they confer survival advantages to the species despite incurring significant costs to the individual and their kin and community.

The reframing of common mental disorders such as trauma from forms of biological breakdown to adaptive changes of a (typically) functionally intact biological system profoundly challenges our understanding of human psychology and its vulnerabilities. Mental health and mental illness are increasingly public and contested global issues - and it's in such context that new scientific perspectives of trauma and common mental illnesses are emerging - consistent with new models that place the social environment and evolutionary-developmental biology at the centre of trauma, common forms of mental illness and recovery ${ }^{28}$.

\section{Trauma}

\section{Etymology}

Trauma is an old word. Its origins can be traced back to the Proto-Indo-European (PIE) language and dialects spoken in the Neolithic Age; some 6000 years ago by inhabitants of the Ponto-Caspian steppeland that stretches over nearly a million square kilometres between the Caspian and Black Seas in what is now called Eastern Europe $^{29,30}$.

The PIE root word is trau associated with piercing, from which came the Greek word trauma meaning 'a wound, hurt or defeat' ${ }^{29}$.

\section{Dictionary Definitions}

The meaning of trauma in western thought has split into 3 categories: 
1. physical trauma - a body wound or shock produced by sudden physical injury, as from violence or accident ${ }^{31}$

2. psychological trauma - a disordered psychic or behavioural state resulting from severe mental or emotional stress or physical injury ${ }^{32}$

3. an event, force or agent that causes physical and/or psychological damage - for example 'war-trauma', 'sexual-abuse trauma' or 'bushfire trauma' ${ }^{13}$

Essentially then, the 3 meanings of trauma are:

1. severe bodily harm

2. severe psychological harm

3. the thing that causes severe harm to bodies and minds (or the self as a whole)

\section{Clinical Definitions}

In Medicine trauma is defined as:

1. physical injury

2. psychological or emotional damage

In practice, trauma is used interchangeably with injury - which is defined:

injury [L. injuria] harm or hurt; usually applied to damage inflicted on the body

by an external force. Called also trauma and wound ${ }^{34}$

\section{Psychology and Psychiatry}

The Diagnostic and Statistical Manual of Mental Disorders Fifth Edition (DSM-5), published by the American Psychiatric Association, describes a range of conditions which are associated with exposure to traumatic or stressful events including reactive attachment disorder, post-traumatic stress disorder (PTSD) and adjustment disorders ${ }^{35}$.

Trauma is not explicitly defined in the DSM-5, but rather various forms of trauma are used as criteria to define different categories of mental disorder. Thus the DSM-5 defines PTSD by:

Exposure to actual or threatened death, serious injury, or sexual violence in one (or more) of the following ways:

1. Directly experiencing the traumatic event(s)

2. Witnessing, in person, the event(s) as it occurred to others 
3. Learning that the traumatic event(s) occurred to a close family member or close friend. In cases of actual or threatened death of a family member or friend, the event(s) must have been violent or accidental to qualify

4. Experiencing repeated or extreme exposure to aversive details of the traumatic event(s) (e.g., first responders collecting human remains: police officers repeatedly exposed to details of child abuse)

5. The DSM-5 criteria specifically excludes exposure through electronic media, television, movies, or pictures, unless this exposure is work related ${ }^{35}$

There are significant problems associated with carving up trauma into diagnostic criteria in this way. Other forms of demonstrable trauma - such as racism, gender discrimination, family abuse and bullying supposedly do not cause PTSD, even though they may reliably produce many of its features ${ }^{36-38}$. Furthermore, it is known that prolonged failure of caregiving experienced as a child (in contrast to catastrophic events) produce some of the most severe (persistent and profound) ${ }^{\text {ie }}$ post-traumatic mental illnesses that manifest in adults ${ }^{39-41}$.

The International Classification of Diseases (ICD-10) produced by the World Health Organisation (WHO) also defines trauma in the context of post-traumatic stress disorder as a stressful event or situation (of either brief or long duration) of an exceptionally threatening or catastrophic nature, which is likely to cause pervasive distress in almost anyone ${ }^{42}$.

The American Psychological Association's Dictionary of Psychology $2^{\text {nd }}$ ed. Defines trauma as:

trauma n. 1. any disturbing experience that results in significant fear, helplessness, dissociation, confusion, or other disruptive feelings intense enough to have a long-lasting negative effect on a person's attitudes, behaviour, and other aspects of functioning. Traumatic events include those caused by human behaviour (e.g., rape, war, industrial accidents) as well as by nature (e.g., earthquakes) and often challenge an individual's view of the world as a just, safe, and predictable place. 2. any serious physical injury, such as a widespread burn or a blow to the head. ${ }^{43}$

The Dictionary goes on to define a traumatic disorder as any disorder that results from physical or psychological trauma ${ }^{43}$. But there is no specific entry nor definition for the term 'psychological trauma'. ${ }^{43}$

In summary, the orthodox model of 'psychological trauma' (as distinguished from physical injury) is, essentially - exposure to severe adverse event(s) that drive persistent and debilitating consequences in mind and body. 
The Features of Evolved Adaptive Responses (FEAR) model proposes that 'psychological trauma' is primarily a neurophysiological rather than a psychological phenomenon - that is - trauma and other common forms of mental illness originate in evolved adaptive functions of the human nervous system and not primarily in dysfunctions of neural wiring, neurochemistry or cognitive and emotional processing. According to this framework, the term 'psychological trauma' is problematic because of the implication that the origin and effective treatment of the condition of trauma lie in the psychological domain. In order to redress this, the term 'Functional trauma' is proposed which serves to

- necessarily distinguish the condition from physical trauma

- situate the condition within the domain of neurophysiology and developmental biology rather than as a primarily psychological phenomenon

- signal a theoretical and clinical reorientation from targeting brokenness towards working with evolved adaptive functions 


\title{
Part Two: Brokenness
}

Mental disorders are pervasive in the population; they do not breed true across generations in families; they show little causal specificity; and they do not simply go away, instead often morphing with time into other, different conditions. ${ }^{44}$

\section{Time doesn't heal all wounds}

\begin{abstract}
When post-traumatic stress disorder (PTSD) first made it into the diagnostic manuals, we only focused on dramatic incidents like rapes, assaults, or accidents to explain the origins of the emotional breakdowns in our patients. Gradually, we came to understand that the most severe dysregulation occurred in people who, as children, lacked a consistent caregiver. Emotional abuse, loss of caregivers, inconsistency, and chronic misattunement showed up as the principal contributors to a large variety of psychiatric problems. ${ }^{39}$
\end{abstract}

The scientific and clinical understanding of trauma has been transformed by research into childhood experiences of adversity. Robust evidence demonstrates that early-life adversity strongly predicts the leading causes of adult disease and death. Adverse Childhood Experiences (ACEs) including maltreatment, domestic violence, parental separation or divorce, parental mental illness, parental substance abuse, and parental incarceration have been linked to $>40$ negative outcomes in adult life, including chronic diseases such as heart disease and cancer, addictions, mental illness, reduced life opportunities, and decreased life expectancy ${ }^{45}$. Because they drive persistent and profound negative life consequences, ACE's can arguably be classed within the general category of traumas.

Importantly, research into early childhood adversity began with a landmark study of a large population of people with high socio-economic status and whose negative health and life outcomes manifested even if they'd never engaged in risky behaviours such as smoking, drinking or overeating. Consequently, two basic mechanisms have been proposed to explain how early adversity drives the incidence of adult diseases and all-cause mortality - the dysregulation model and the evolved adaptation model.

\section{Dysregulation}

Certain specific bodily processes such as body temperature and blood $\mathrm{pH}$ are maintained within tight limits. This process of tight control to maintain a set-point is called homeostasis. But in general, organisms demonstrate variability in their states rather than defending a particular physiological state. This is because organisms must continuously adapt to the demands of dynamic environments in order to survive, 
grow and reproduce. Development and evolution represent trajectories of adaptation. The process of continuously coordinating and changing physiological processes to adapt to environmental change is called allostasis. The physiological flexibility and changeability of allostasis enables the organism to adapt to environmental challenges such as noise, crowding, isolation, hunger, extremes of temperature, danger, and microbial or parasitic infection ${ }^{46}$.

The dysregulation model proposes that cumulative stress and adversity erode the organism's basic adaptive capacity, by creating a burden - an 'allostatic load' - that it cannot coordinate physiological recovery from ${ }^{46}$. Consequently, runaway stress mechanisms such as inflammation provoke a host of pathophysiological changes in the body and brain that produce disease and increase the risks for death from all causes $^{47}$.

It's estimated that some $95 \%$ of research about stress in psychology, neuroscience and medicine follows an implicit dysregulation model ${ }^{48}$. Dysregulation research has been tremendously successful in mapping the systemic and pathological sequelae of stress, adversity and traumatic experiences. The model of stress-related dysregulation builds on an underlying model that defines and frames mental illness in terms of disorder, dysfunction and disease. But there are significant problems and explanatory gaps that confront the dysregulation-disorder-dysfunction-disease or 'breakdown' model of trauma and mental illness.

\title{
Scale
}

In societal context, mental illness is an immense and escalating problem. In 2018, the World Health Organization determined that depression alone has become the leading cause of illness and disability worldwide. ${ }^{49}$

\begin{abstract}
"As an economist, I want to emphasise that mental health is a significant problem for our economy- as significant as, often more significant than, tax or microeconomic reform... Many people do not get the support they need and governments get poor returns on substantial investment... The economic or GDP gains from better mental health would dwarf most of the gains - often modest ones - being talked about in current economic reform debates."
\end{abstract}

$$
\text { Professor Allan Fels }{ }^{50}
$$

In Australia, the direct and indirect costs of 6 of the most common severe mental illnesses (depressive disorders, anxiety disorders, drug and alcohol-use disorders, schizophrenia and bipolar disorder) is estimated at \$98.8 AUD billion per annum, which is $\sim 6 \%$ of the national GDP ${ }^{51}$. In the United States, the direct costs of mental disorders (public and private spending) exceeded \$201 USD billion per annum, greater than all other conditions (such as heart disease, physical trauma and cancer) ${ }^{52}$ 


\section{Etiology}

etiology noun

1. cause, origin specifically: the cause of a disease or abnormal condition

2. a branch of knowledge concerned with causes specifically: a branch of medical science concerned with the causes and origins of diseases ${ }^{53}$

Despite decades of research and billions of dollars in funding, no biological causes have been identified for any of the common forms of mental illness ${ }^{54-59}$. Current expert opinion ${ }^{60}$ asserts that the etiology of mental illness is exceedingly complex and that unravelling causality is perhaps "one of the most difficult tasks that medical scientists will ever face" because

1. mental illnesses are the product of multiple convergent factors

2. they are multigenic - in which any contributing genetic predisposition exerts only a small effect

3. the environment exerts a heavy influence

\section{Biomarkers}

In the future, we hope to be able to identify [psychological] disorders using biological and genetic markers that provide precise diagnoses that can be delivered with complete reliability and validity. Yet this promise, which we have anticipated since the 1970s, remains disappointingly distant. We've been telling patients for several decades that we are waiting for biomarkers. We're still waiting. ${ }^{61}$

Since the latter half of the nineteenth century, objective clinical tests have become central to the practise of western medicine. Currently, there are over 3000 standardised laboratory tests and hundreds of clinical devices (such as the electrocardiogram - EKG and electroencephalogram - EEG) available for the diagnosis and monitoring of disease ${ }^{62}$. Clinical tests rely on detecting specific biological signatures of the state of the organism - of its health, disease or response to therapy - called biomarkers. A biomarker is defined as a biological measure that provides information about the state of a normal biologic process, pathogenic process, or pharmacologic response to an intervention ${ }^{63,64}$. Example of biomarkers in current clinical use include C- Reactive Protein (CRP) for detecting inflammation and Brain Natriuretic Peptide (BNP) for detecting acute heart failure. Yet, not withstanding massive research investment, no validated clinical biomarkers have been demonstrated for any mental illness ${ }^{64-67}$. 


\section{Comorbidity}

Comorbidity is the occurrence of 2 or more disorders or diseases in the same person, either simultaneously or over the lifespan ${ }^{68}$. In adults, comorbidity is the norm rather than the exception ${ }^{69}$ - with $32 \%$ of working-age adults in high-income countries, 38\% of people in developing countries and $62 \%$ of people in underdeveloped countries experiencing 5 or more chronic health conditions ${ }^{70}$.

Comorbidity can produce multiplicative effects - leading to increasing severity and complications, iatrogenic effects (where treatment for one condition worsens the other) and worsening prognosis ${ }^{71}$ and is trending towards increasing prevalence likely due to factors such as increasing lifespan and increasing social inequality ${ }^{72}$.

Psychiatric disorders are characterised by high rates of comorbidity, both with physical diseases and with other mental illnesses ${ }^{73}$. In the US, data from the National Comorbidity Study of 65,244 adults aged between 15-54, revealed that 50\% of respondents reported receiving a diagnosis of mental illness during their lifetime, and of those $79.4 \%$ reported the diagnosis of 2 or more mental disorders ${ }^{74}$. Data from the subsequent 2001-2003 National Comorbidity Survey Replication (NCS-R) collected from a nationally representative sample of adults aged 18 and over, revealed that nearly $75 \%$ of adults reported experiencing 1 or more chronic physical or mental conditions in the previous 12 months and of the people with mental disorders, between $84-98 \%$ also met the criteria for additional physical and mental conditions ${ }^{25}$.

Psychiatric comorbidities are strongly correlated ${ }^{75,76}$ - that is common mental illnesses cluster together, for example Major Depressive Episode and General Anxiety Disorder or Alcohol Dependence and Drug Dependence ${ }^{77}$. 'Pure cases' where a person fits the criteria for a single mental disorder are in fact atypical ${ }^{78}$. A massive study of 5.9 million people in Denmark, spanning 83.9 million person-years of amassed health data, examined comorbidity between the 10 major classes of mental disorders defined in the ICD-10 such as early-onset behavioural disorders, substance use disorders, personality, anxiety disorders, psychotic disorders and mood disorders and revealed that comorbidity between the different kinds of mental disorders is pervasive and that all general classes of mental disorder increase the risk of developing any other class of mental disorder ${ }^{61}$.

Increasingly, it appears that the pervasive comorbidity of mental disorders is due not to the prevalence of multiple psychiatric conditions, but rather, is an artefact of inaccurate systems of classification (DSM-5 and ICD-10) producing apparent rather than actual comorbidity - mental disorders that typically cluster together may not actually be separate conditions but different clinical manifestations of a common underlying condition ${ }^{77,79-81}$. 


\section{Nosology}

"No sharp distinction can be drawn between mental health and mental disease; a large collection of material shows a gradual and not an abrupt transition from the normal state to pathological states." ${ }^{195}$

A Study of Association in Insanity, Kent GH \& Rosanoff AJ, 1910

"depressive symptoms are present in almost all psychiatric disorders [and] every sign or symptom seen in schizophrenia occurs in other psychiatric and neurological disorders"

\section{Kaplan \& Sadock's Concise Textbook of Clinical Psychiatry ${ }^{82}$}

nosology, noun

1. a classification or list of diseases

2. a branch of medical science that deals with classification of diseases ${ }^{83}$

The currently accepted systems for classifying mental disorders suffer from critical problems when applied in clinical and research settings ${ }^{84-86}$ :

1. Diagnostic categories such as schizophrenia or post-traumatic stress disorder are internally heterogenous

2. Low reliability of the diagnoses in clinical settings

3. Excessively high rates of comorbidity

4. Lack of treatment and prognostic specificity

5. Failure to scientifically validate diagnostic categories

There are two major diagnostic systems for mental disorders in current clinical use the DSM-5 (published by the American Psychological Association) and the ICD-10 (published by the World Health Organisation). Both are, in actuality, quite similar, being minor modifications of the previously published DSM-III ${ }^{58}$.

At its core, the DSM-5 is a categorical system of classification, wherein mental disorders are presented as separate and discrete entities such as Major Depressive Disorder and Schizophrenia. However, accumulating evidence points towards common genetic, environmental, developmental and physiological factors that cut across the DSM-5's diagnostic categories ${ }^{87}$.

As a consequence, the editors of the DSM-5 have been compelled to acknowledge that the boundaries between many supposedly different disorders are more fluid than previously recognised, and that

"many symptoms assigned to a single disorder may occur, at varying levels of severity, in many other disorders...Although some mental disorders may have 
well-defined boundaries around symptom clusters, scientific evidence now places many, if not most, disorders on a spectrum with closely related disorders that have shared symptoms, shared genetic and environmental risk factors, and possibly shared neural substrates..."

Furthermore, concerted research efforts and billions of dollars of funding have failed to validate the model of any mental disorder as a discrete disease with a unitary (single and simple) pathological cause such as a single gene or 'chemical imbalance ${ }^{158,82}$.

"...the goal of validating these syndromes and discovering common etiologies has remained elusive. Despite many proposed candidates, not one laboratory marker has been found to be specific in identifying any of the DSM-defined syndromes. Epidemiological and clinical studies have shown extremely high rates of comorbidities among the disorders, undermining the hypothesis that the syndromes represent distinct etiologies. Furthermore, epidemiological studies have shown a high degree of short-term diagnostic instability for many disorders. With regard to treatment, lack of treatment specificity is the rule rather than the exception." 85

and furthermore:

"The boundaries of these categories have not been predictive of treatment response." ${ }^{88}$

The categorical system of mental illness classification has led to exponentiating diagnostic complication. For example, the classification of Borderline Personality Disorder consists of nine diagnostic criteria of which a minimum of five need to be identified for the diagnosis of BPD. Consequently, there are 256 possible different ways that BPD may present. Unfortunately, this is a small number of variants compared to other disorders - for example there are 636,120 ways to have posttraumatic stress disorder ${ }^{89}$.

The disorders identified in the DSM have been defined by committees using expert opinion and consensus which is rated as the lowest grade of scientific evidence ${ }^{90,91}$.

"The mental disorders included in DSM-5 have not gained their official status through any rational process of elimination. They made it into the system and have survived because of practical necessity, historical accident, gradual accretion, precedent, and inertia — not because they met some independent set of abstract and universal definitional criteria. No surprise then that the DSM disorders are something of a hodgepodge, not internally consistent or mutually exclusive." 58

and 
"More than once, important decisions that affected the final outcome of a DSM categorization system were made at the level of the Board of Trustees of the APA. The claim that the DSMs represent the best science possible is not credible when the final arbitrator of decision making is not a scientific body but the leaders of an organization with profit motives." 92

As a consequence of such problems, the peak federal agency funding mental health research in the United States, the National Institute of Mental Health (NIMH) has effectively abandoned the DSM nosology and embarked on an ambitious quest to create a new psychiatric nosology by translating neuroscience research into protocols for the clinical treatment of mental disorders and the promotion of mental health ${ }^{93}$.

NIMH's proto-nosology, called the Research Domain Criteria (RDoC) rests on the assumptions that:

1. Mental illnesses are disorders of yet to be identified brain circuits (wiring disorders) rather than neurological lesions

2. Disordered brain circuits will be identified with currently existing and emerging technologies such as fMRI

3. Big Data from sources such as genomics and connectonomics will yield biomarkers or biosignatures for mental disorders ${ }^{88}$

The RDoC represents a radical reformulation of the psychiatric model of mental illness. The prevailing biopsychosocial model frames mental illness holistically as an outcome of phenomena occurring in the biological, behavioural, psychological and social dimensions of human existence ${ }^{94}$. In contrast, the RDoC model posits that mental disorders are fundamentally disorders of brain structure and function that affect cognitive, emotional and behavioural domains ${ }^{95}$. NIMH's parent organisation the National Institutes of Health has bet big on this premise, investing over \$USD 30 billion in the Human Connectome Project to study patterns of neural connectivity in health and sickness in the belief that mapping the functional anatomy of the brain's networks will reveal the roots of psychopathology ${ }^{86}$. However, a decade after its introduction, the RDoC model remains a set of assumptions and a conceptual framework that have yet to be validated ${ }^{96,97}$.

\section{Transdiagnostic features}

Classically, psychiatric nosology has focused on the features which distinguish supposedly different mental disorders. However, the failure to scientifically validate the diagnostic categories of the DSM-5 and related ICD-10, coupled with the ubiquitous comorbidity of mental disorders observed in clinical practice, is driving increasing attention towards the shared features of mental disorders as potentially critical factors in their etiology and resolution ${ }^{98}$. 
'Transdiagnostic features' are common processes observed across many different categories of mental illness ${ }^{99}$. For example, for many years, the orthodox psychiatric perspective was that a panic attack is a unique and specific feature of panic disorder, however, it's now recognised that panic attacks are pervasive and can occur in any form of mental disorder ${ }^{99}$.

Transdiagnostic features have been described in the scientific literature since around the turn of the $21^{\text {st }}$ century ${ }^{99,74}$, however there are yet no definitions of the terms 'transdiagnostic' or 'transdiagnosis' in current editions of the APA Dictionary of Psychology ${ }^{43}$, nor in Oxford, Merriam-Webster or Taber online medical dictionaries. ${ }^{100-102}$

Transdiagnostic processes may be causal, consequential or both (through positive feedback loops) $)^{74,99}$ and have been identified in many of the dimensions of mental illness - including neurological, physiological, behavioural and social. In the cognitive domain - transdiagnostic processes observed across the range of common adult mental disorders include ${ }^{74}$ :

1.Attentional processes - such as selective attention towards perceived threats

2.Memory processes - such as recurrent memories

3.Reasoning processes - such as distorted, disordered or biased analysis, hypothesis and interpretation

4.Thought processes - such as recurrent or perseverating thinking

5.Behavioural processes - such as exaggerated threat mitigation and avoidance

Other transdiagnostic processes and phenomena include:

1.Insomnia - conservatively identified in $41-53 \%$ of mental disorders ${ }^{103}$

2.Systemic Inflammation - inflammatory (cytokine) processes are pervasive in mental disorders and have been described in depressive disorders, bipolar disorder, anxiety disorders, autism, schizophrenia, cognitive decline, and neurodegenerative diseases ${ }^{104}$

3.The Gut-Brain-Microbiome axis - high rates of comorbidity are reported between gastrointestinal and psychiatric disorders ${ }^{105}$. Multidirectional communication between the central and peripheral nervous systems, the gastrointestinal tract and the microbiome - the bacterial colonisation of the human body (in a healthy body, bacteria outnumber human cells by 10 to 1$)^{106}$ is an evolved and vital component of human physiology and health. Animal models reveal that the health of the microbiome influences behaviour, neurochemistry, immunity and development ${ }^{107}$. Dysbiosis (meaning adverse change in the constituency and functions of the microbiome) is described in a broad range of mental disorders including depression, schizophrenia, bipolar disorder, autism spectrum and attention-deficit hyperactivity disorder ${ }^{108}$

4.Heart Rate Variability (HRV) - healthy physiological processes such as heartbeat demonstrate complexity and chaos (unpredictable non-random variability) rather than metronomic invariability ${ }^{109}$. High tonic HRV is robustly associated with positive physical and psychological health, while low resting and/or over-reactive HRV is 
observed in a broad range of psychological and physical disorders - including schizophrenia, depression, PTSD, heart disease, diabetes, physical trauma, epilepsy and cancer ${ }^{110-113}$, indicating that HRV is likely a biomarker of critical systemic regulatory functions

5.Negative symptoms - meaning attenuation of goal-creation and goal-pursuit and reductions in prosocial behaviour, pleasure and enjoyment and emotional and verbal expressivity are observed in schizophrenic-type, personality, depressive, anxiety, bipolar, autism and post-traumatic stress disorders ${ }^{114}$

\section{Treatment Effectiveness}

Current evidence-based treatments - including Drug therapy, Cognitive Behavioural Therapy, Counselling and GP visits - when added together avert just 13\% of the disability burden (years lived with disability - YLD's) of common mental illnesses. However, it's also been shown that only a fraction of people with mental disorders ever receive treatment - yet even when researchers modelled the improbable scenario of every affected person in the population getting unrestricted access to evidencebased treatment, $60 \%$ of the burden of common mental illnesses would nevertheless remain unaverted - indicating the significant gap between the social magnitude of mental illness and the state of scientific knowledge and current evidence-based treatments ${ }^{115}$.

Pharmacological treatment for mental illnesses has increased globally over the previous 2 decades driven by increasing use of antidepressant and antipsychotic medication $^{116}$. In the US, $86 \%$ of outpatient visits to a psychiatrist resulted in prescription of psychotropic medications (such as antidepressants, antipsychotics, mood stabilisers, and sedative-hypnotics), in $60 \%$ of visits the patient received a prescription for 2 or more psychotropic drugs and in 33\% of visits the patient received a prescription for 3 or more psychotropics ${ }^{117}$.

Schizophrenia is a relatively common mental disorder and is typically characterised as a severe and lifelong illness ${ }^{118,119}$. Beginning in 1973, the World Health Organization initiated large-scale longitudinal multi-country studies investigating the presentation, diagnosis and outcomes of schizophrenia. The unanticipated finding was that people with schizophrenia in poorer 'developing' countries such as India, Colombia and Nigeria, had significantly better outcomes (such as course of illness, current functioning, clinical status and life-course) than people in richer 'developed' countries such as the UK, US, Denmark and Russia, despite only 16\% of the patients in developing countries being maintained on antipsychotic medication compared to $61 \%$ of patients in developed countries. The best outcomes were recorded in India, where only $3 \%$ of patients received antipsychotics and the worst outcomes were recorded in Moscow with the highest rates of antipsychotic use ${ }^{118}$ and these outcome differences were shown to persist over the short (2-5 yr), medium (15yr) and long terms (26 yr) ${ }^{120}$. Nor are such outcomes confined to 'developing' countries. During the 
1970's-1980's Western Lapland in Finland had one of the highest rates of schizophrenia in Europe - 2-3 times more than the rest of Finland and Europe. An innovative therapy called Open-Dialog was developed to meet the need- a form of combined family therapy and sociotherapy involving the patient, their family, therapists, case-workers and even members of the community including employers and police. In a longitudinal study of 75 people with first-episode psychosis treated with the Open-Dialog method, $67 \%$ were never exposed to treatment with antipsychotic drugs and had experienced no relapses at 5 year follow-up, 79\% were asymptomatic and $73 \%$ were working or in school. Long-term outcomes for psychosis in Western Lapland have become the best in the western world ${ }^{118}$.

The use of antidepressants has become prolific. In Australia, antidepressants are the most commonly used medications, taken by $10 \%$ of the adult population daily and at a rate that has doubled since 2000 , to be amongst the highest in the world ${ }^{121}$. In the USA, between 2011-2014, the CDC estimated 12.7\% of people age 12 and older took antidepressants. Over $60 \%$ of these people had taken antidepressants for 2 years or longer, and $25 \%$ had taken for $>10 \mathrm{yrs}^{122}$. However meta-reviews of clinical research reveal that antidepressant medications have low to zero clinical effectiveness compared to placebo ${ }^{123-126}$ and systematic reviews reveal that long-term users of antidepressants have worse or no better outcomes than non-users ${ }^{127,128}$.

More than 4 decades of psychiatric drug development has not reduced the morbidity or mortality of mental disorders ${ }^{125,129}$ and there is significant evidence of severe and widespread harm caused by the major classes of psychiatric medications including antidepressants ${ }^{130}$, antipsychotics ${ }^{131}$ and sedatives ${ }^{132}$ including chronic brain impairment, dementia, self-harm, suicidality and violence, birth defects, impaired child development and metabolic and sexual dysfunctions ${ }^{133,125,134}$. It is conservatively estimated that adverse consequences ('side effects') of these 3 classes of psychotropic drugs cause the preventable deaths of more than half a million people aged 65 and over every year in the western world ${ }^{125}$.

\section{The Pharmacological Revolution}

The discovery of psychiatric drugs, beginning with the use of chlorpromazine (marketed under the tradenames of Thorazine and Largactil) as an antipsychotic in $1952^{135}$, was heralded as a 'Psychopharmacological Revolution' ${ }^{134}$ in the treatment of mental illness. Akin to the discovery of broad-spectrum antibiotics such as streptomycin which had led to the successful treatment of infectious diseases that had plagued humankind for centuries such as pneumonia, diphtheria and tuberculosis, the new psychiatric medications were proclaimed as the 'magic bullets' that would finally cure mental disorders. An entire academic research industry was created to study drug action and the emerging field of 'biological psychiatry', backed by funding from the 
US Congress and the National Institute for Mental Health ${ }^{134}$. By 1967, 1 in 3 adults in the US had filled a prescription for a psychoactive drug ${ }^{118}$.

A story of scientific progress in the understanding and treatment of mental illness was extolled to the public and in medical education. According to the US Surgeon General's first ever report on mental health in 1999 - prior to the drug revolution, "Treatments were not effective. Early treatment was no more successful in preventing patients from becoming chronically ill in the early 20th century than it was in the early years of the previous century." ${ }^{\text {"136 }}$. The discovery and development of antipsychotic, antidepressant and anti-anxiety drugs created "a variety of treatments of well-documented efficacy for the array of clearly defined mental and behavioural disorders that occur across the life span.”"136,118.

\section{Insulin vs Alcohol Models of Psychoactive Drug Action}

The 'disease model' of psychiatric drug action claims that the psychiatric drugs work by reversing a deficit or imbalance in brain chemistry ${ }^{118,134}$. The chemical imbalance model of mental disorders has become the prevalent cultural model of mental disorders in western countries ${ }^{137,138}$ and reinforced by official information disseminated to the public - for example, The British Royal College of Psychiatrists' public information sheet entitled 'Depression' suggests that "two... neurotransmitters (serotonin and noradrenalin) are particularly affected" in depression and claims that "antidepressants increase the concentrations of these two chemicals at nerve endings, and so seem to boost the function of those parts of the brain that use serotonin and noradrenalin” (Royal College of Psychiatrists 2006). The American Psychiatric Association (APA) says that "antidepressants may be prescribed to correct imbalances in the levels of chemicals in the brain" (American Psychiatric Association 2005). On psychosis or schizophrenia the Royal College of Psychiatrists (RCP) claims that there are "abnormalities in the biochemistry of the brain" and "an imbalance in brain chemistry” (Royal College of Psychiatrists 2004). The APA suggests that antipsychotic medications "help bring biochemical imbalances closer to normal” (American Psychiatric Association 1996).”134. In the United States and New Zealand, medications including antidepressants are advertised directly to the public, with television viewers in the US exposed to up to 9 ads for medication per day ${ }^{139}$. Ads for the most prescribed antidepressants such as Zoloft and Paxil emphasise the notion that the drugs correct chemical imbalance ${ }^{140,141}$.

In fact, despite massive academic and commercial research efforts, there is no validated evidence that any common mental disorder is caused by neurochemical imbalance. Instead, it appears that all current psychotropic drugs act on the brain in essentially the same way - not by targeting and correcting specific chemical imbalances but by disrupting molecular pathways and profoundly altering brain function ${ }^{118,141}$. Consequently, psychotropic medications produce similar effects in 
'normal' people as in people diagnosed with a mental disorder - because the therapeutic mechanism of current psychiatric drugs is more akin to the way alcohol disrupts brain function in both normal and abnormal subjects rather than the way therapeutic insulin produces an effect by correcting a metabolic deficiency in people with diabetes but produces no such effect in non-diabetics. According to the 'drugmodel' of psychiatric drug action - it is the global disruption of brain function not the correction of neurochemistry, which is the principle therapeutic effect of psychiatric medications - that is, when doctors and patients preference the altered functioning of a drug-disrupted brain to the functioning of a 'mentally disordered' brain - the treatment is deemed successful ${ }^{118,134}$.

Speaking about the mechanism of action of the commonly prescribed SSRI antidepressants, one neuroscience researcher observed:

These drugs alter the level of synaptic transmission beyond the physiologic range achieved under [normal] environmental/biological conditions. Thus, any behavioural or physiologic change produced under these conditions might more appropriately be considered pathologic, rather than reflective of the normal biological role of 5-HT [serotonin.] ${ }^{118}$.

And according to another medical researcher:

Psychotropic drugs don't fix a chemical imbalance, they cause it, which is why it is so difficult to come off the drugs again. If taken for more than a few weeks, these drugs create the disease they were intended to cure. We have turned schizophrenia, ADHD and depression, which were often self-limited diseases in the past, into chronic disorders because of the drugs we use. ${ }^{131}$

\section{Doctoring the Science and Manufacturing Ignorance}

In 1953, the giants of the tobacco industry (American Tobacco, Benson and Hedges, Philip Morris and U.S. Tobacco) in coordination with the public relations firm Hill \& Knowlton, initiated a campaign against the mounting scientific evidence of harms caused by tobacco. Dubbed by investigators as 'the tobacco strategy', its endgame was to obstruct regulation of smoking by manufacturing ignorance on a grand scale systematically and strategically engineering widespread disinformation, confusion and doubt about the evidence of tobacco harms ${ }^{142}$.

"Doubt is our product, since it is the best means of competing with the 'body of fact' that exists in the mind of the general public"

Brown \& Williamson Tobacco Corporation (B\&W) internal memo, $1969^{143}$

The tobacco strategy consisted of 5 mechanisms: 
1. Exploit the inherent uncertainty of all scientific knowledge to create an impression in the minds of public and policy-makers that, despite increasingly robust evidence of significant harm, definitive proof had not been established and 'more research needs to be done'

2. Finance friendly research

3. Recruit distinguished scientists

4. Create echo chambers in the media and in the academic sphere - for example through advertising campaigns and sponsored events

5. Attack the credibility of unfavourable research

The tobacco strategy was highly successful - the industry did not lose any court cases until the 1990s and tobacco was not regulated as an addictive drug in the US until $2009 .{ }^{142}$

In the 1990s evidence-based medicine began as a movement to transform clinical decision-making and the practice of medicine away from reliance on tradition, anecdote, opinion and theory, towards reliance on evidence from high-quality research (such as randomised controlled trials and observational studies) combined with clinical expertise and the needs and wishes of patients ${ }^{144}$. Evidence based medicine can be defined operationally as an architecture of information, that is designed to get the right evidence to the right doctor at the right time ${ }^{145}$ in order to create positive medical outcomes. But, more than 2 decades after its introduction, evidence-based medicine is confounded by significant issues including:

- Systematic corruption of the biomedical evidence-base by vested interests

- An increasingly unmanageable volume of clinical information

- Research that is of increasingly marginal clinical benefit

- Facilitation and formation of orthodoxy and dogmatism that mediate against inquiry and innovation

- Inflexible evidence-base rules and clinical guidelines that produce management-based care not patient-based care

- Evidence based clinical guidelines that fail to encompass the multimorbidity especially common in older people ${ }^{144}$

Medical information and knowledge is a product of a complex system - the healthcare system - which can be described as an infrastructure that produces basic research, products and services that are used to create medical outcomes. But what is created by healthcare systems is not simply an outcome of the needs and actions of patients, clinicians and communities but also of the private financial motivations of industry, political interests ( ${ }^{\mathrm{eg}}$ from lobby groups), academia ( ${ }^{\mathrm{eg}}$ career and publication incentives) and the shifting priorities of funding agencies. Competing and divergent interests coupled with ineffective or compromised regulation, produce perverse incentives that make the production and dissemination of objective scientific evidence a highly problematic endeavour ${ }^{146,145}$. 
In the US, currently $64 \%$ of all biomedical research and development funding comes from private industry, with just $22 \%$ coming from government. In contrast, China is committing large governmental resources to developing biomedical research and development capabilities ${ }^{147}$.

Worldwide, over $\$ 240$ billion USD is invested in health research and development per year, resulting in an estimated 1 million research publications per year ${ }^{148}$. The production of the biomedical evidence base is hugely inefficient - generating $>85 \%$ 'waste' meaning research that doesn't add to but detracts from the quality of scientific evidence ${ }^{149}$, in other words producing useless information by:

- studying unimportant or low priority issues

- research in a vacuum - failure to contextualise and demonstrate how the study adds value to the evidence-base

- poor experimental design and high risk of bias

- selective publication - publishing in part or failure to publish; particularly of problematic results

Although the problems driving the massive inefficiency of biomedical research are deemed surmountable and correctable, the system as a whole is resilient against reform due to perverse incentives that prioritise profit over ideals about human welfare and the integrity of scientific evidence. ${ }^{145}$

The practical implications of the deluge of wasteful and polluted evidence are considerable - to keep up with published articles relevant to the field of general practice, for example, a doctor would be required to spend more than 600 hours per month merely to skim through the latest literature ${ }^{150}$.

Medicine today is practised using drugs that have come onto the market over several decades, supported by evidence that has been gathered since at least the 1970s. We now know that this entire evidence base has been systematically distorted by the pharmaceutical industry, which has deliberately and selectively withheld the results of trials whose results it didn't like, while publishing the ones with good results. ${ }^{145}$

Biased reporting is a widespread phenomena in the medical literature and is not confined to drug research, but has also been identified in research studies of surgical, diagnostic and preventative medical interventions ${ }^{151}$.

The tobacco strategy became the de facto model for manufacturing widespread ignorance by manipulating the scientific evidence-base and influencing public perception ${ }^{142}$. The preponderance of evidence points to the systematic 'gamification' 
of the biomedical evidence-base - meaning that every stage of the production and dissemination of biomedical research has become a strategic 'attack-surface' ${ }^{152}$ for the competitive manipulation of information and perception at the expense of the integrity of scientific evidence ${ }^{153,145}$.

\section{Known Vectors of Attack against the Biomedical Evidence Base ${ }^{145,154,155}$}

Outright fraud

Financial ties to researchers, academics, and clinicians

Withholding publication of trials that failed to show benefits or showed harms

Using unlikely test subjects

Testing interventions against rubbish comparisons

Changing trial lengths to disguise negative outcomes

Trials using too few subjects

Trials that ignore subjects that drop out

Trials that change the main outcome after they've finished

Subdividing subjects into special groups after the trial ends

Disguising marketing as clinical trials

Pretend it's all positive in the published article

Advertising directly to patients

Celebrity endorsement

Misleading official factsheets

Biased Clinical Practice Guidelines

Widening disease categories

Astroturfing (using compromised or fictitious grassroots organisations to articulate and advocate messages that advantage industry)

Advertising to doctors

Marketing 'off-label' - outside of approved use

Drug reps

Ghostwriters

Publication planning (strategic communication and marketing of industry-sponsored research by private communications agencies)

Industry sponsored events

Approximately $75 \%$ of the articles published in leading medical journals including The Lancet, the New England Journal of Medicine (NEJM) and the Journal of the American Medical Association (JAMA) are industry funded. 2/3rds of this research is conducted by private contracted research organisations - which allow the sponsor explicit control over study design, data analysis methods and publication ${ }^{156}$.

Financial relationships between industry, researchers, and academic institutions are pervasive. A 2002 survey revealed that $81 \%$ of the authors of clinical practice guidelines (which purport to support best practice and best evidence in clinical decision-making) had financial links to pharmaceutical companies ${ }^{155}$ and that too 
often clinical guidelines had become marketing tools for industry ${ }^{157}$. Unsurprisingly, systematic review reveals that research funded by industry is systematically biased to produce results favourable to industry - commercially funded research is 5.3 times more likely to support the sponsor's products than independent studies ${ }^{158}$.

Psychiatry has been at the epicentre of concerns about financial conflicts of interest in medicine due to media revelations of ghostwriting of published articles, failures to disclose financial ties and vested interests in the promotion of psychotropic drugs. Conflicts of interest in psychiatry are problematic because clinical decision-making, guided by the DSM-5 nosology, has global public impacts in areas such as insurance, health and social welfare policy and law. Research reveals a clear connection between industry funding and the production of outcomes favourable to the funder ${ }^{156,159}$.

The DSM-IV was published in 1994, and after receiving criticism that it contained no disclosure information about the financial ties of its authors and contributors, the American Psychiatric Association instituted a mandatory disclosure policy, revealing that 57\% of DSM-IV task force members had financial ties to industry. By 2013 and the publication of DSM-5, this had increased to $69 \%$ of the DSM-5 task force members reporting financial ties to pharmaceutical companies ${ }^{160}$.

A critical consequence of a compromised and corrupted biomedical evidence-base is widespread public health illiteracy - that is - manufactured social ignorance which is not merely the absence of validated knowledge (ignorance) but the systematic production of false knowledge (disinformation) ${ }^{142}$ by mechanisms ${ }^{146}$ including:

- Biased funding of research

- Biased reporting in medical journals

- Biased reporting in health pamphlets

- Biased reporting in the media

- Financial conflicts of interest

- Defensive medicine (the practice of medicine to avoid the risk of litigation rather than to promote patient welfare)

- Clinician's failure to critically evaluate information sources

\section{Psychotherapies and Cognitive Behavioural Therapy}

There are more than 600 brands (different modalities) of psychotherapy ${ }^{161}$. Historically, many therapists subscribe to a 'pure form' or brand of therapy (therapeutic allegiance) and eschew integration of different modalities (eclecticism). Rivalry between therapeutic camps is pervasive and the different modalities do battle in a 'dogma eat dogma' competition for practitioner's attention and affection and access to continuing professional development revenue ${ }^{162}$. Many interventions have 
never been validated in controlled clinical trials and the field is rife with unvalidated constructs and theoretical edifices ${ }^{161}$.

Nevertheless, the absolute efficacy (meaning positive effects of intervention compared to no treatment measured in clinical studies) of psychotherapeutic interventions is considerable and has been robustly replicated since the 1970 's ${ }^{163}$.

The investigation of relative efficacy - that is, the superiority or inferiority of one psychotherapeutic modality compared to another in clinical trials, began earlier, in 1936. Researchers have described a 'Dodo bird verdict', from the book Alice in Wonderland, in which the Dodo, presiding over a race where contestants start and end when they want, declares "Everybody has won and all must have prizes". In psychotherapy, the Dodo bird verdict is perhaps best defined as "All methods of therapy when competently used are equally successful"163. Although highly contested, over the following decades, high-quality studies and meta-analyses (a form of statistical analyses that seeks to increase explanatory power by aggregating the results from many pooled studies) have validated the Dodo bird verdict, demonstrating close to zero or statistically insignificant differences between psychotherapeutic brands in the treatment of a broad range of mental illnesses ${ }^{163,164}$.

Modern medicine has made great advances in treating conditions such as infectious disease and physical trauma and in the development of medical technologies for imaging, surgery and pharmacotherapy. Consequently, a 'Medical model' of healthcare has come to emphasise the primacy of technology, knowledge and technical competence in driving clinical outcomes ${ }^{163,165}$. However, evidence indicates that common human factors including empathy, understanding, shared goals, positive regard, genuineness and forming a therapeutic alliance with the client can produce large clinical benefits, and in the psychotherapies - common human factors have been shown to produce greater therapeutic benefit than the particular intervention used. Therapist effects have also been demonstrated in pharmacotherapy where a clinician with high 'human factors' competency delivering a placebo drug produces a significantly better patient outcome than a clinician of lesser human factors competency delivering a drug of proven efficacy ${ }^{163}$.

One psychotherapeutic brand - Cognitive Behavioural Therapy (CBT) - has achieved hegemonic status - meaning that it has come to dominate state-funded education, training and service-delivery in western developed nations including the United Kingdom, Australia, New Zealand, Sweden and the United States ${ }^{166}$. CBT is enthusiastically promoted by governments to address a broad gamut of complex social problems including mental illness ${ }^{167}$, rehabilitation of criminal offenders ${ }^{168,169}$ and return to work following psychological or physical injury ${ }^{170}$.

Sweden provides a prototypical example of the mechanism by which CBT arose to its current dominance in the so-called 'therapy-wars' ${ }^{171,161}$. In the early 2000's, seeking to 
address the massive economic and social costs of people falling out of work or not ever entering the workforce, most commonly due to mental illness - the Swedish government was swept up in the fervour of western governments to embrace 'evidence-based' mental health policy. Clinical guidelines were drawn-up to standardise mental health practice. Topping the list of evidence-based psychotherapies at the time was $\mathrm{CBT}$ and consequently the state restricted all funding for training of clinicians and treatment of clients to CBT, excluding all other forms of psychotherapy. 7 years later, after spending the equivalent of more than $\$ 700$ million USD, the Swedish National Audit Office audited the program and reported that it had had no significant effect on the return-to-work outcomes of people disabled by depression or anxiety, and concluded:

"Steering towards specific treatment methods has been ineffective in achieving the objective."

Subsequently, the Socialstyrelsen, Sweden's National Board of Health and Welfare, determined to end the CBT monopoly and broadened it's support of validated psychotherapies $^{175}$.

Cognitive Behavioural Therapy is extensively promoted as the 'gold standard' of psychotherapies for reasons of the sheer volume of studies that make up its evidencebase and the numbers of academic programs and professionals devoted to its practice ${ }^{176}$. The American Psychological Association's Division 12 Task Force on Psychological Interventions lists CBT as the only treatment with "strong research support" in almost $80 \%$ of all the mental disorders included ${ }^{177}$. The oft-cited claim that CBT is 'the most researched form of psychotherapy ${ }^{176}$ makes especially salient the observation that quantity of evidence does not equate to quality of evidence. The widespread adoption of CBT into international clinical guidelines and subsequently government mental healthcare policies in many western developed nations for a broad range of common mental illnesses, was based on what is increasingly recognised to be low quality evidence - including many studies with significant design flaws and high-probability of bias ${ }^{177-181}$.

The replicability crisis in psychological research ${ }^{182,183}$, further unravels CBT's claim to evidence-based superiority. Replicability in science essentially means that independent researchers, repeating the methodology of a prior study, can reproduce its results and conclusions. The reproducibility of published research is a basic determinant of the validity of scientific knowledge. In 2015, researchers reported that of a sample of 100 of the most cited studies published in high-ranking psychology journals, of which $97 \%$ reported positive findings, the findings of only $36 \%$ could be replicated. Furthermore, of the studies and their results that were successfully reproduced, the effect size (essentially a measure of the power of the phenomenon or intervention under observation) was about half that reported in the original study ${ }^{184}$. 
The issue of research replicability is not confined to psychology but is endemic in all fields of scientific research where the majority or even the vast majority of published research claims may be false due to factors including research design flaws, perverse incentives and researcher and publication biases ${ }^{185,186}$. Increasing awareness of such systemic threats is driving initiatives such as Open Science to not only safeguard the integrity of scientific research but to 'evolve' science itself ${ }^{187-189}$.

If the evidence-based merit cited by its proponents does not convincingly validate as the basis for the hegemony of CBT, what then is the mechanism for its dominance in research, regulations, guidelines and practice? Using historical comparison, there are a number of striking parallels between the ascension of CBT in the $21^{\text {st }}$ century and the trajectory of Scientific Forestry in the $18^{\text {th }}$ century as international 'gold standards' of 'sustainable management' and 'evidence-based best practice' in their respective domains: 


\begin{tabular}{|c|c|c|}
\hline & CBT & Scientific Forestry \\
\hline $\begin{array}{l}\text { Origins in Economic } \\
\text { Ambitions of the State }\end{array}$ & $\begin{array}{l}\text { Reduce productivity losses due } \\
\text { to mental incapacitation }\end{array}$ & $\begin{array}{l}\text { Appropriation of forests for } \\
\text { industrialisation }\end{array}$ \\
\hline Elite Lobbying and Alarmism & $\begin{array}{l}\text { 'The Depression Report' issued } \\
\text { by the London School of } \\
\text { Economics declares that the } \\
\text { mentally incapacitated are a } \\
\text { bigger drain on the economy } \\
\text { than the unemployed, and that } \\
\text { evidence-based psychotherapy } \\
\text { - primarily CBT - will reduce } \\
\text { the burden by half }\end{array}$ & $\begin{array}{l}\text { German Academics claim that } \\
\text { the poor are ransacking the } \\
\text { forests and introduce } \\
\text { 'sustainable' Scientific Forestry } \\
\text { as the solution }\end{array}$ \\
\hline $\begin{array}{l}\text { Unvalidated Claims of } \\
\text { Effectiveness }\end{array}$ & $\begin{array}{l}\text { CBT would pay for itself by } \\
\text { improving return-to-work } \\
\text { outcomes }\end{array}$ & $\begin{array}{l}\text { Forestry Science would } \\
\text { preserve the forests for nation- } \\
\text { building }\end{array}$ \\
\hline $\begin{array}{l}\text { Convergence with vested } \\
\text { interests }\end{array}$ & Therapy Wars & Class conflict \\
\hline Hegemony & $\begin{array}{l}\text { Monoculture replaces plurality } \\
\text { of psychotherapy research, } \\
\text { education and practice }\end{array}$ & $\begin{array}{l}\text { Monoculture replaces forest } \\
\text { biodiversity }\end{array}$ \\
\hline $\begin{array}{l}\text { Promotion as the 'Gold } \\
\text { Standard' }\end{array}$ & $\begin{array}{l}\text { CBT widely adopted by } \\
\text { governments of western } \\
\text { developed countries }\end{array}$ & $\begin{array}{l}\text { Scientific Forestry widely } \\
\text { adopted throughout western and } \\
\text { colonised nations }\end{array}$ \\
\hline $\begin{array}{l}\text { Failure to Deliver Primary } \\
\text { Outcomes }\end{array}$ & $\begin{array}{l}\text { National Audit Office of } \\
\text { Sweden concludes that despite } \\
\text { heavy state investment, CBT is } \\
\text { ineffective at improving return- } \\
\text { to-work outcomes }\end{array}$ & $\begin{array}{l}\text { Waldsterben - the death of the } \\
\text { forests as a consequence of the } \\
\text { 'sustainable management' } \\
\text { practices of Scientific Forestry }\end{array}$ \\
\hline Restoration & $\begin{array}{l}\text { Sweden returns to supporting } \\
\text { plurality of psychotherapies }\end{array}$ & $\begin{array}{l}\text { German forestry pioneers } \\
\text { restoring forest biodiversity }\end{array}$ \\
\hline
\end{tabular}

Thus, in comparative historical perspective, the hegemony of CBT appears to be driven not by the preeminence of its scientific evidence but by the intersecting ambitions of a coterie of technical, political and economic stakeholders. A clinical 'gold-standard' which is thus compromised by private vested interests and which drives therapeutic guidelines and regulatory policies to restrict funding, research and access to the constellation of effective psychotherapeutic approaches, fails to serve the public good.

\section{The Nature of Mental IIIness}

Psychiatric disorders remain historical-clinical syndromes of unknown etiology. ${ }^{190}$ 
The essential nature of mental illness remains mysterious ${ }^{191,192}$. In 1883, the German physician Emil Kraepelin, credited as the founder of modern scientific psychiatry, published his 'Compendium der Psychiatrie: Zum Gebrauche für Studirende und Aertze' (Compendium of Psychiatry: For the Use of Students and Physicians), proposing that psychiatric conditions originate in biological and genetic malfunctions. Yet, more than 130 years later, the science of mental illness is described by leading researchers as being in a 'still-nascent state' ${ }^{193}$. Consequently, even formulating a scientific definition of mental illness continues to be problematic ${ }^{194}$. The current biomedical definition of mental illness given in the DSM5 attempts to traverse multiple terrains of definition, description and differentiation from the historical misuse ${ }^{82}$ of psychiatric diagnosis:

A mental disorder is a syndrome characterized by clinically significant disturbance in an individual's cognition, emotion regulation, or behavior that reflects a dysfunction in the psychological, biological, or developmental processes underlying mental functioning. Mental disorders are usually associated with significant distress or disability in social, occupational, or other important activities. An expectable or culturally approved response to a common stressor or loss, such as the death of a loved one, is not a mental disorder. Socially deviant behavior (e.g., political, religious, or sexual) and conflicts that are primarily between the individual and society are not mental disorders unless the deviance or conflict results from a dysfunction in the individual, as described above. ${ }^{35}$

However, the putative (presumed) dysfunctions in the domains underlying mental functioning remain unidentified and unvalidated. ${ }^{35,54}$

The 'new kid on the block' - the National Institutes of Mental Health's Research Domain Criteria (RDoC) abstains from attempting to define mental disorder, claiming that it is as 'agnostic to defining mental disorder' as it is agnostic to the unvalidated diagnostic categories of the DSM-5 and ICD $10^{193}$, which it was created to supercede. This would appear to be an enlightened position for an institution tasked with the scientific investigation of mental disorders. However the RDoC is neither atheoretical nor truly pluralistic in its reconsideration of the nature of mental illness, instead reiterating a biomedical model of mental illness and its basic axioms of biological brokenness, the primacy of the individual and focus on downstream causes over the social and economic determinants of health and disease.

The historical quest to uncover the aetiology of mental illness - its ultimate pathological cause - that traces its origins to Germany in the late $19^{\text {th }}$ Century ${ }^{195}$ (around the time that the adverse consequences of German Scientific Forest Management were becoming apparent ${ }^{9}$ ), that mobilises research programs throughout the world, involving leading academics and laboratories and using ever more sophisticated analytical technologies - has for about 150 years - failed to validate biological lesions, disorders, dysfunctions or dysregulations in the aetiology of any of the common forms of mental illness. 
The biomedical model is thus both poorly explanatory and poorly effective as a framework for understanding and treating mental illnesses. Critical ontological problems confront the biomedical modelling of mental illness:

1. The failure to validate biological brokenness in the aetiology of any of the common forms of mental illness.

2. The low effectiveness of currently prescribed evidence-based treatments for mental disorders, predominantly based on models that presume brokenness, when measured at the population level ${ }^{115}$.

3. The tremendous human variability in kinds and presentations of mental illness ${ }^{35}$ leading to an ever-expanding catalog of mental illnesses - with 541 categories of mental disorder described in the DSM-5, an increase from the 383 mental disorders described in the DSM-IV ${ }^{92}$.

4. For the fraction of mental illness categories for which there are defined diagnostic criteria (151 of the 541 disorders in DSM-5) - the phenomenological method ${ }^{196}$ of matching a patient's clinical presentation to authoritative collections of signs and symptoms (the DSM's 'syndromes') leads to a combinatorial explosion of diagnostic possibilities ( ${ }^{\text {eg }} 256$ ways to have Bipolar Disorder and 636,120 ways to have PTSD) $)^{89}$.

5. Yet for all the phenomenal variability and diagnostic complication, comorbidity (cooccurrence) between mental disorders is ubiquitous ${ }^{44}$ and bidirectional (meaning that the occurrence of any kind of mental disorder can lead to the co-occurrence of any other kind ${ }^{76}$. Arguably, current classifications of mental disorders bear only a weak relationship to clinical and epidemiological reality.

6. Robust and accumulating evidence indicates that there is a common structure or 'risk architecture' of shared factors underlying supposedly different kinds of mental illnesses ${ }^{197}$ - so that what appear as many different forms of mental disorders may actually be points along the spectra of a relatively few underlying conditions ${ }^{80}$.

7. A growing plethora of transdiagnostic features described across common mental disorders, indicating the presence of common pathways in the etiology and/or continuity of supposedly different forms of mental illness and the potential for transtherapeutic mechanisms for effective interventions across diagnostic categories.

8. Clear evidence of the principal role of social factors such as sense-of-control as the fundamental causes of population health and disease outcomes.

As a consequence of the significant unresolved and unaddressed problems in biomedical modelling and clinical interventions, a radically different 'complex systems' modelling of common psychological conditions is arising from the intersections of diverse fields including social science, evolutionary-developmental biology, neurophysiology, social genomics and social epidemiology. 


\section{Part Three: Complexity}

\section{Wicked Problems}

Many people [with psychological issues] think they need treatment ... when what they really need most is support ${ }^{198}$

In 2006, the number of obese and overweight people in the world overtook the number of people who are underweight and malnourished. In the industrialised western nations including Australia, the UK and the USA, obesity has become a population-level problem. In Australia, government data reveals that about $1 / 4$ of children and adolescents and about $2 / 3$ of all adults are overweight or obese ${ }^{199}$. A focus on treating individuals, behavioural change and preventing adverse clinical outcomes has failed to stem exponentiating rates of obesity. ${ }^{200}$

Massive and escalating problems such as obesity and mental illness are increasingly characterised as 'complex' and 'wicked' problems - meaning problems that are highly resistant to resolution using current approaches ${ }^{201}$. Wicked problems may be colloquially described as problems where the current solutions are killing us ( ${ }^{\text {ie }}$ producing static or worsening outcomes) and where improving the efficiency of existing solutions is killing us more comprehensively, by misdirecting our attention, resources and actions away from critical and disruptive thinking, engaging diverse perspectives, developing new models and driving creativity and innovation ${ }^{202}$. Technically, wicked problems have been characterised ${ }^{201}$ as problems that:

- are difficult to define

- have many causes and dependencies

- are unstable

- attempts at solution produce unpredictable consequences

- have no clear and permanent solution

- may be socially complex

- don't sit within the remit or scope of any one organisation

- require change-agents to change their mental models, culture and behaviour

It is becoming increasingly apparent that the current models and predominant frameworks for understanding and addressing social needs are maladapted to the challenges of solving complex wicked problems such as mental illness:

A traditional bureaucracy, divided into vertical silos, in which most of the authority for resolving problems rests at the top of the organisation, is not welladapted to support the kinds of process necessary for addressing the complexity and ambiguity of wicked problems. Bureaucracies tend to be risk averse, and are intolerant of messy processes. They excel at managing issues with clear boundaries rather than ambiguous, complex issues that may require experimental and innovative approaches. ${ }^{201}$ 


\section{Complex Systems}

The word complex comes from the Latin word plectere meaning to weave and entwine. In complex systems, many simple parts are irreducibly entwined in a functional whole and there are critical properties of the whole that cannot be found in the parts - in the same way that the taste of sugar cannot be found in its constituent elements of carbon, hydrogen and oxygen. The study of complexity is itself an entwining of many different fields.

Historically, the problems that science seeks to understand can be divided into 3 types $^{203,204:}$

- Simple systems that consist of a small number of variables or components and which can be completely or almost completely mathematically described - for example systems such as clocks, cars, digital computers and machines.

- Disorganised complexity in which there is a very large number of variables or parts that behave apparently randomly in relation to one another such as the trillions of particles that make up a gas cloud or a fluid. Systems of disorganised complexity cannot be described and predicted with simple mathematical formulas, but can be described, modelled and predicted using the tools of probability and statistics.

- Organised complexity in which relationships between large numbers of diverse variables drive a system's behaviour - for example economic systems, the immune system, networks, organisations, cities, forests and ecosystems. Systems of organised complexity cannot be described nor predicted using simple mathematical formulas nor statistics, but can be modelled using the tools of complexity science including interdisciplinary model-building, non-linear mathematics, adaptive heuristics, genetic algorithms and big data.

Research in public health, biomedicine and social science has historically relied on and in general continues to apply the quantitative methods and statistical tools (such as regression) developed for modelling systems of disorganised complexity to the investigation of systems of organised complexity.

There is increasing recognition that wicked problems and complexity require new forms of analysis, models and methods. People and communities are complex adaptive self-organising systems and health and disease emerge from self-organising processes occurring at multiple levels and scales.

Starting with the recognition that it is systems that generate health disparities and population health, complex systems approaches allow us to appreciate that the production of health inequalities does not rest only on complicated systems with lots of pieces, but instead on complex systems involving multiple interacting factors with dense, and sometimes nonlinear, feedback. As such, health inequalities, as complex systems, cannot be easily reduced to independent components with analyses of the isolated impacts of single components. 
[To be continued ( 3more paragraphs here)]

\section{Public Health}

It seems incontrovertible that as a wicked (massive, complex and social) problem, mental illness is a public health issue. Public health has been defined as:

the science and art of preventing disease, prolonging life, and promoting health through the organised efforts of society ${ }^{205}$

Public health is a whole-of-society endeavour concerned with the health of populations. Notwithstanding its elegant definition, the historical reality of public health is neither simple nor straightforward - in fact public health is an ideological and political battleground ${ }^{206,207}$. In the orthodox narrative, public health is modelled as a function of biomedical healthcare, however the weight of historical and contemporary evidence indicates that the contribution of biomedical healthcare to public health is marginal to limited. Consequently, instead of situating public health under the dominion of biomedical healthcare, public health is more appropriately modelled as a systemic outcome - a product of a complex network of interactions: ${ }^{208-}$ 210

Figure: Public health is a systemic outcome of interacting processes and domains.

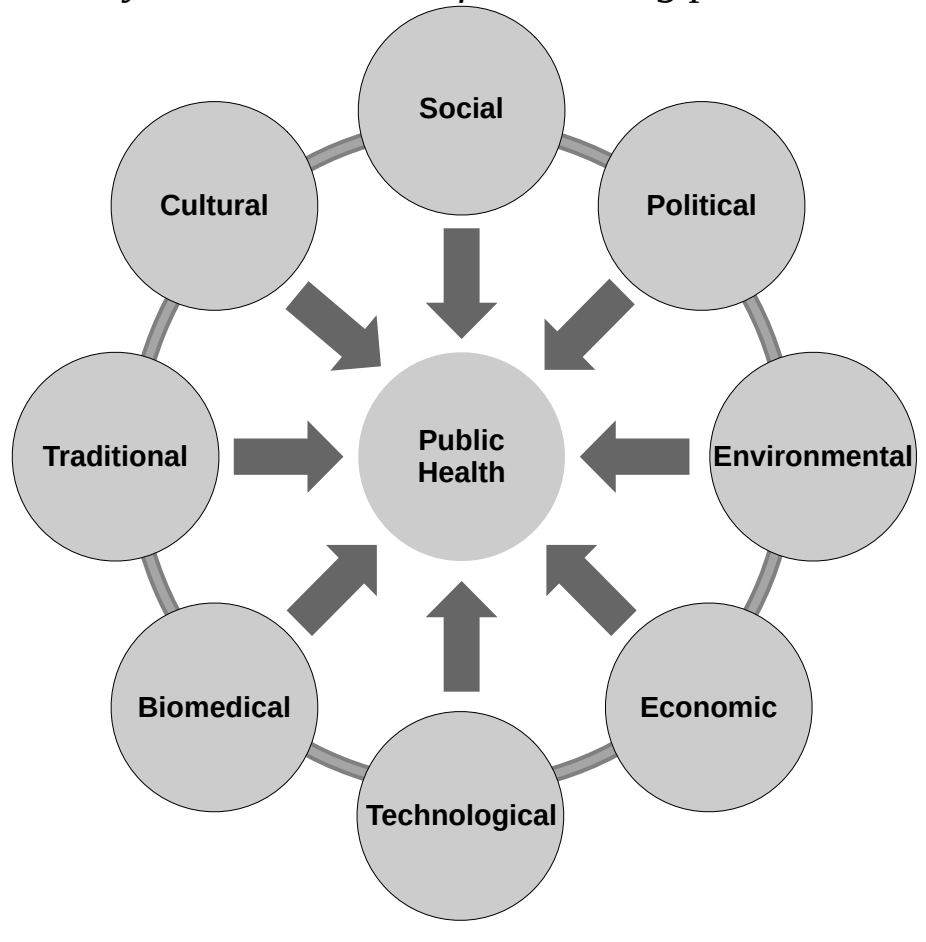

Illuminating the complexity - the evolutionary history - of public health can serve to 
1. Broaden understanding of mental health by differentiating the proximal and fundamental causes of health and disease

2. Cast a critical light on orthodox narratives about the relationship between biomedical healthcare and population health

3. Develop more complete and useful models of complex social problems such as drug addiction, persistent health inequalities and mental illness which current healthcare and policy approaches are failing to ameliorate

\section{The $1^{\text {st }}$ Wave}

5 overlapping waves have been described in the development of public health in western industrialised countries ${ }^{211,212}$, all which reach into the present, continuing to shape and impact population health. Historically, each wave seems to arise as social and technological change together with the passage of time render the limitations of previous waves of development apparent. Using the UK as the prototypical example, the first wave occurred between about 1830-1900, as a response to adverse consequences of industrialisation ${ }^{213}$ - including the demise of the feudal social contract, the erosion of community and kinship networks, slums, overcrowding, poverty, malnutrition, lack of sanitation, increasing alcoholism and crime - which factored together to create a pathogenic environment for the spread of diseases including tuberculosis, cholera and typhus.

There was little to no government expenditure on basic urban infrastructure such as sewers, clean water and paved roads in keeping with a dominant political ideology of 'laissez-faire, laissez passer' effectively translated as 'everyone for themselves ${ }^{213}$. The predominant explanation for disease, particularly as it affected communities of poor and marginalised people, was that people were suffering the just consequences of their own moral degeneracy, intemperance and incivility ${ }^{214,215}$. Gradually, as pioneering work revealed the connections between unsanitary living and working environments and patterns of sickness and disease, public acceptance of the rationale for government investment in basic infrastructure for the common good, grew.

Heavy investment in public infrastructure and services commenced in this period including the construction of reservoirs and sewers and the forming of modern police forces and municipal authorities. In the 1870's, the city of Birmingham pioneered a program of buying, building and running local utility companies (decried by opponents as 'gas and water socialism') in order to ensure critical infrastructure development and public accessibility and affordability. Having proved the viability of this new 'political economy', the program went on to be replicated in all other major cities and towns in England over the next 3 decades $^{213}$.

Importantly, infrastructure investment coupled with parallel improvements in housing, living and working conditions brought about major reductions in mortality and the prevalence of diseases - by fixing their 'upstream causes' - before their 'downstream causes' such as bacteria were even discovered ${ }^{211}$ - a stark illustration of 
the power of addressing fundamental causes to change downstream population health consequences - proverbially understood as 'putting a sign at the top of the cliff' obviating the need to park an ambulance at the bottom of the cliff.

\section{The $2^{\text {nd }}$ Wave}

The second wave of public health development, from about 1890-1950, was characterised by the 'coming of age' of science and technology - transforming a broad range of social sectors including medicine (with the introduction of the germ theory of disease), engineering, transport and manufacturing and the inception of the academic discipline of urban planning aiming to design and develop urban environments for the greater public good ${ }^{216}$. The figure of the professional expert arose in this era - a specialist in a narrow domain of knowledge, whose opinions could guide effective public policy and decision-making. A prevailing idealism originating in the Enlightenment, upheld the power of science, technology, and reason to pave a royal road for human progress ${ }^{211}$.

\section{The $3^{\text {rd }}$ Wave}

The Beveridge Report, published in 1942, in the middle of the Second World War, marked the defining moment in the third wave of public health reform - received rapturously by the press and public, turning its author into an overnight public hero, with people queuing up to obtain copies the night before the official release, selling over half a million copies - an unprecedented record for what was essentially a government technical document and going on to influence post-war debates on social reform throughout the english-speaking world. The report identified 5 'giant evils'217 that were unvanquished in society despite material progress, advances in medicine and technology, cadres of professional specialists and experts and preceding public health reforms ${ }^{211,218}$ :

\section{Want (caused by poverty)}

2. Ignorance (caused by a lack of education)

3. Squalor (caused by poor housing)

4. Idleness (caused by a lack of jobs and/or skills)

5. Disease (caused by inadequate health care provision)

Social welfare in Britain originated in the Poor Law of 1834, based on a principal of social deterrence which required claimants to be incarcerated in a local 'workhouse', where families were split up and those capable of work were subject to hard labour. The more prosperous populace typically considered the poor as moral and social degenerates and outcasts; subscribing to a Malthusian worldview wherein the poor represented an unsustainable drain on resources. Thus voluntary imprisonment in the poorhouse brought shame and disgrace, which many sought to avoid. In the early $20^{\text {th }}$ century, incarceration was abandoned in favour of more cost-effective 'means-testing' (intrusive investigation and evaluation of a claimant's life circumstances in order to 
qualify for social welfare) while retaining the ethos of social deterrence and thus the stigma and disgrace of falling in need and poverty persisted as instruments of social control $^{219}$.

But public sentiment began to shift during WWII, which had thrown England together in a way that WWI had not - forcing one half to see how the other half lived - as 1.5 million school-age children, mothers with under-school-age children and teachers from cities and towns were billeted in the countryside, together with mass movements of workers to the new manufacturing centres of the national war-effort and conscription and migration from bombed out areas disrupted traditional class boundaries and threw social inequality into stark relief. For example, the introduction of wartime food-rationing, presented by the government and widely perceived by the public as a necessary hardship - significantly reducing the amount, variety and quality of available foodstuffs ${ }^{220}$ - also produced unanticipated and startling health outcomes:

the families in that third of the population of Britain who in 1938 were chronically undernourished had their first adequate diet in 1940 and 1941 ... [due to the distribution of free food under the rationing system, after which] the incidence of deficiency diseases, and notably infant mortality, dropped dramatically. ${ }^{221}$

Overall British public health - measured by reduced infant mortality and increased adult life-expectancy - significantly improved as a consequence of food rationing ${ }^{220}$.

The collision of ordinary realities caused by the war-effort changed social consciousness by revealing how many people, perhaps especially children, were falling through the gaps left by the operations of the economic market, charity and existing social policy.

Having made so many sacrifices in which so many had lost so much, there was emerging a shared determination in the country that if victory could be achieved the peace that followed should be won for the benefit of all. ${ }^{222}$

This shift in public sentiment cannot be characterised as a trend toward socialism defined as seeking collective ownership of the means of production ${ }^{223}$, but rather as an emerging recognition and acceptance of concepts of universal social rights, social justice and governmental responsibility for the welfare and health of the whole of society.

The third wave of public health represents a societal recognition, enacted in subsequent public policy, that ensuring the provision of basic determinants of health and wellbeing is a social responsibility in a civil society ${ }^{224}$. Comprehensive social security would become coupled to the liberal market economy - effectively renewing the post-war social contract. Buoyed by a new social consciousness, third wave 
public health and social policy led to the creation of the National Health Service (NHS) providing health care to all as a basic right, universal social insurance (where contributions made whilst working - guaranteed social benefits for workers and their families in the event of sickness, accident, unemployment and in old age), paved the way for universal education, massive post-war housing projects and a broad range of reforms to ameliorate poverty and unemployment ${ }^{219,222,221}$.

\section{The $4^{\text {th }}$ Wave}

The fourth wave of public health arose in the 1960's, in the final decade of the Golden Age of Capitalism. The OPEC Oil Crisis in October 1973 heralded recessions in the USA and UK that brought to an end decades of post-WWII economic expansion. Western developed nations began to transition from manufacturing economies into service and knowledge driven economies, coupled with broad social change including falling fertility rates, changing gender roles, increasing divorce rates, increasing consumer choice, declining trust in institutions and more transient social ties ${ }^{211}$.

Dramatic increases in population health, measured by changes in life expectancy, mortality and fertility, had occurred in western industrialised nations between the $18^{\text {th }}$ to the $20^{\text {th }}$ centuries ${ }^{211,187}$. In the UK in 1800 , life expectancy at birth was about 40 years and by 1950, it was 68.7 years $^{225}$. In the US, between 1900 and 1973 there was a $69.2 \%$ decrease in all-cause mortality ${ }^{226}$, with life expectancy from birth increasing from $\sim 47$ years in 1900 to 70 years by $1960^{225}$. Described as the 'demographic transition' - changing population life expectancy, mortality and fertility is consistently observed worldwide in response to economic development ${ }^{227}$. But there is also another consistent feature of changing global population health - persistent health inequality.

In the UK, The Black Report of 1977-80 revealed large differences in mortality and morbidity between social classes that had been widening since the 1950's, despite the introduction of universal free access to medical healthcare via the NHS ${ }^{228}$. Large inequalities in health have been repeatedly demonstrated across European countries also despite more universal access to healthcare ${ }^{229}$. UK data from the 1980's to 2016 demonstrate that health inequalities between the most affluent and the most deprived communities continue to widen, driven by a diverse range of causes (including respiratory diseases, heart disease, stroke, diabetes, cancers, dementias, intentional and unintentional injuries and all other causes) ${ }^{230}$.

Beginning in the late 1970's, under the conservative Thatcher regime, there was an ideological shift in government policy away from post-war principles of social justice, social welfare, full employment and free public services towards a social prescription of deregulated labour and financial markets (the unfettering of the 'free market'), reduced taxation for businesses and the most wealthy, dismantling of public 
programs and institutions oriented to social justice and privatisation of formerly public utilities and services ${ }^{231}$ - a prescription that would, it was asserted, drive economic growth and, in turn, advance human health and welfare ${ }^{206}$. The consequences of these policies on public welfare were dire, driving massive increases in unemployment, income inequality and health inequalities ${ }^{231}$ including a neardoubling of mortality differences between the most prosperous and most deprived households $^{228}$. For example, in the 10 year period between 1981-85 to 1991-95, for adults aged between 15-44 living in the most economically prosperous regions of the country, overall mortality fell by an average of $17 \%$. But for adults of the same age living in the most economically deprived regions, overall mortality increased by up to $44 \%$.

Britain has experienced some of the fastest growth in income inequality in the developed world and by the late 1990s had some of the highest levels of poverty seen within Europe. ${ }^{228}$

The fourth and currently dominant wave of public health development was brought about by the convergence of 3 key factors:

1. the failure of the existing public health strategies to solve widening health inequalities - for example mortality and morbidity inequalities due to the so-called 'non-communicable diseases' (such as heart-disease, chronic respiratory disease, diabetes, cancer and mental illness)

2. the coming of age of clinical epidemiology with the capacity to identify individuals and groups 'at-risk' of adverse health outcomes

3. an ideological shift in social policy which sought to wind back the commitment of the state to social welfare including universal healthcare and return the onus for health on to the individual

\section{The Era of Individual Risk}

The fourth wave originated as a reorientation of public health policy and interventions away from principles of universal social welfare, justice and equality towards characterising individuals and groups considered to be 'at-risk' of adverse health outcomes and identifying and addressing the risk factors such as genetic and behavioural risks - related to public health problems ${ }^{232,233}$, under the justification, since disproven, that this would be more cost-effective ${ }^{206}$.

Similar reorientations of public health modelling and policy occurred in other western developed nations. In the US,

In 1971, Ann Somers published an influential book called Health Care in Transition, addressing the spiralling health care cost issues with a refocused analysis on the root causes of the new leading causes of death: heart disease, 
cancer, stroke, injuries, diabetes, and other lifestyle-related, mostly chronic disease, problems. These root causes she traced to the irresponsibility or inability of poorly prepared individuals. ${ }^{234}$

An exemplary case of fourth wave public health intervention is cardiovascular disease - a blanket-term for a range of diseases of the heart and blood vessels including hypertension (high blood pressure), coronary heart disease (heart attack), cerebrovascular disease (stroke), peripheral vascular disease and heart failure. Despite the rate of death from cardiovascular disease decreasing globally since about $1993^{235}$, cardiovascular disease remains the leading cause of death in the world accounting for more than $30 \%$ of all global deaths, of which $85 \%$ are due to heart attack and stroke. Heart disease is not confined to wealthier nations, at least $3 / 4$ of total deaths occur in low and middle income countries ${ }^{236}$.

In Australia, ischaemic heart disease (coronary artery disease) is the leading cause of death $^{237}$. In the US, the leading cause of death is heart disease, with cancer predicted to become the leading cause by 2020. Overall, the risk of death from both conditions has been declining, with the risk of dying from heart disease declining from the 1960 's and the rates of cancer increasing in the latter half of the $20^{\text {th }}$ century before declining in the 1990 's ${ }^{238}$. Of the $\$ 1.9$ trillion the US spends on medical healthcare per annum, around $12 \%$ or $\$ 236$ billion is spent on the treatment of cardiovascular disease $^{239}$. Heart disease is thus one of the most extensively funded and researched conditions in modern healthcare, studied intensively and internationally since the 1950 's ${ }^{240}$, with the National Institutes of Health in the US allocation of funding to cardiovascular disease research exceeding $\$ 2$ billion per annum since $2016^{241}$.

Massive worldwide research effort has identified about 50 risk factors ${ }^{242}$ for cardiovascular disease, including cigarette smoking, high lipid levels, hypertension, obesity, physical inactivity, and diabetes - the most prominent of which are routinely referred to in medical advice to patients and public health communication. Yet added together, individual biological and behavioural risk factors account for only about $45 \%$ of the total risk for cardiovascular disease ${ }^{240,243,244}$. Thus, the majority of the risk for heart disease is unexplained by individual biological and behavioural risk factors.

A further problem with the risk approach to health improvement is that interventions to change modifiable risk such as, in the case of heart disease, behavioural change interventions to reduce smoking, increase physical activity and improve diet, are largely ineffective at changing risk profiles and health outcomes, even in highly motivated participants ${ }^{240,245}$.

The prototypal example of a public health intervention targeting behavioural change in an at-risk population is the MRFIT study conducted in the US between 1971-1981, which intended to reduce the death rate of men aged 35-57 who had been categorised in the top $10 \%$ of most at risk from coronary heart disease due to their levels of 3 
prominent risk factors - smoking, serum cholesterol and high blood pressure ${ }^{246}$. MRFIT cost more than $\$ 180$ million (more than \$555 million in today's dollars) and has been described as the most rigorous and expensive clinical trial ever developed to educate people and get them to change their behaviour. Researchers medically screened more than 350,000 men in 22 cities across the US. In order to qualify for the study, participants had to be willing to stop smoking, change their diet as advised, take medication for high blood pressure, attend the clinic regularly, bring family members or friends to group discussions about prominent health risks, receive individual counselling, be assessed at minimum every 4 months and commit to participation for 6-8 years of the study duration. 12,866 highly at-risk volunteers were identified, who were then randomly divided into a treatment group and a control group who were simply advised of their at-risk health status and then sent back to their own doctors for usual care. The resultant treatment group thus consisted of carefully selected and highly informed and motivated participants ${ }^{247}$.

The MRFIT treatment intervention was intensive and sustained - each individual was assigned to an intervention team consisting of nutritionists, nurses, physicians, general health counsellors and behavioural scientists, for each of their 3 prominent risk factors. For example, in the dietary arm of the study, participants and their families were shown how to cook low-fat meals, taken to the supermarket and taught to read food-labels and researchers even accompanied them to their homes to prepare meals ${ }^{244}$. Furthermore, specially trained counsellors worked closely with each participant for the entire duration of the study. The overall outcome of the MRFIT study, historically one of the most massive and comprehensive behavioural change interventions, was - according to one of the principal researchers - failure. After 6 years of intervention, there were no statistically significant differences in heart disease rates between the treatment and control groups ${ }^{244}$. Subsequent systematic review of 4 decades of international studies of behavioural interventions aimed at changing 'modifiable risks' for coronary heart disease, reveals that these interventions produce little to no impact in reducing mortality or morbidity ${ }^{245}$.

\section{The $5^{\text {th }}$ Wave}

The fifth wave of public health development is forming as socioeconomic inequalities widen within the 'developed' nations driving worsening population health and increasingly divided health outcomes. Increasing population mortality, measured at the national level, was a rare phenomenon in the $20^{\text {th }}$ century - observed only in the 1900's in countries severely affected by WWI and WWII and in the 1990's in subSaharan African nations with high AIDS prevalence and in Russia after the collapse of the Soviet Union in 1991. In the USA in the $21^{\text {st }}$ century, population mortality is increasing, with all-cause mortality increasing for all ethnic groups. From leading the world in life expectancy in the 1950's ${ }^{225}$ and despite currently spending more on medical healthcare than the rest of the world combined, the US is currently ranked $35^{\text {th }}-60^{\text {th }}$ in the world depending on which mortality indicator is used ${ }^{248}$. In the UK, 
although life expectancy trended upwards between 2001-2016, female life expectancy in the most economically deprived social rankings stalled or reversed in this period - indicating that public health development is bypassing the poorest communities. ${ }^{230}$.

The failure of clinical epidemiology, with its predominant focus on specific diseases and individual biological and behavioural risk factors ${ }^{212}$ to resolve widening health disparities driven by escalating social inequality, is compelling the recognition that improving public health requires addressing the 'upstream' social causes of health and disease.

Figure: Upstream and Downstream Determinants of Health and Disease

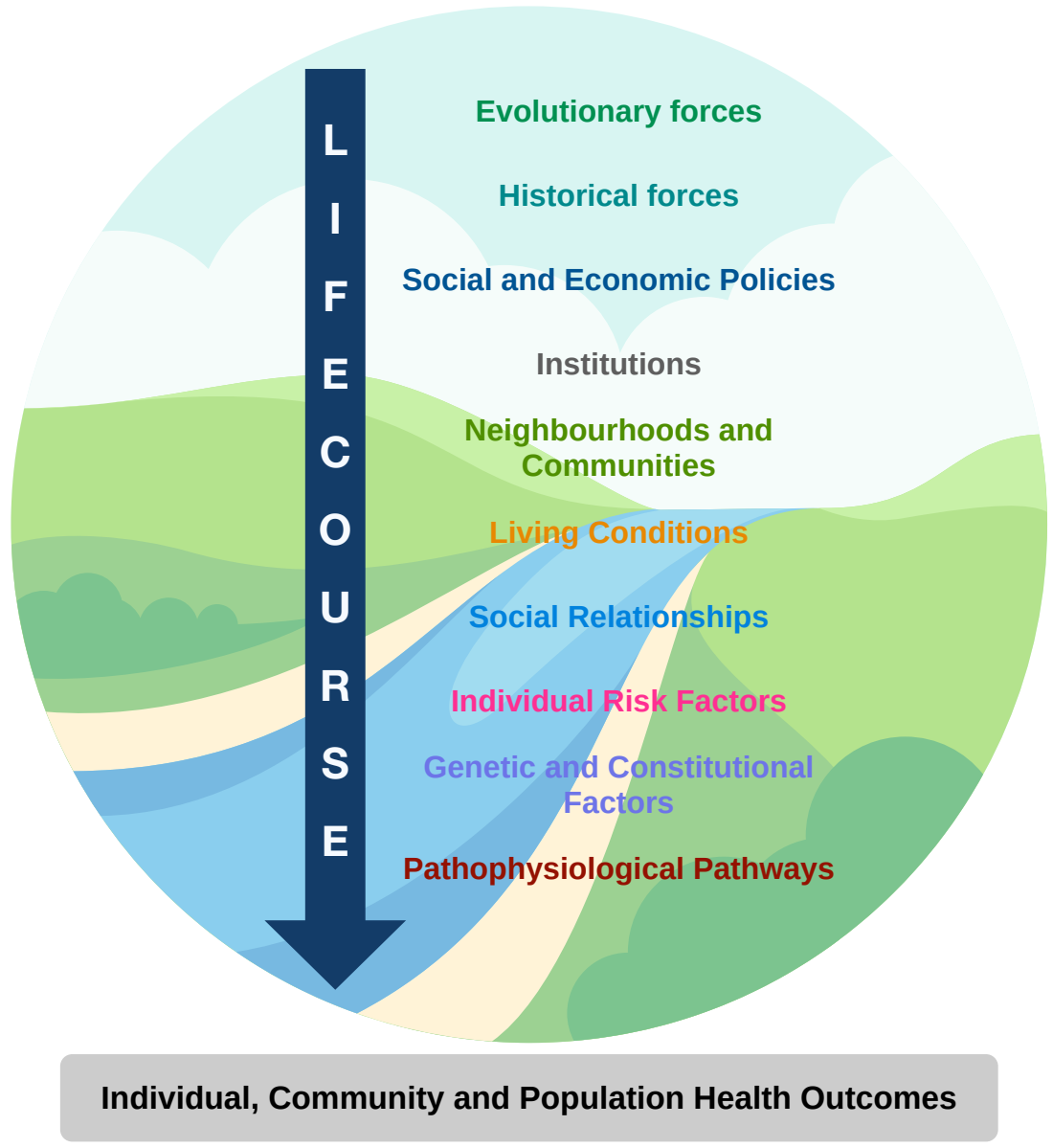

\section{The Environmental Carriers of Disease}

Historically, in the industrialised countries, public health improvement against most of the serious and prevalent infectious diseases came about not by a principal focus on sick individuals and their clinical case management but by understanding and addressing how infectious diseases are transmitted in the population - identifying and intervening at the level of the environmental carrier, for example identifying whether the disease is water-borne, air-borne, food-borne or vector-borne and then changing 
the environment to eliminate the carrier ${ }^{226,240}$, for example by the provision of clean water supplies and sanitation to eliminate cholera. So in the same way, public health improvement in the era of the 'non-communicable diseases' such as heart disease and mental illness, necessitates understanding and addressing the environmental carriers of these diseases - which turn out to be social factors. But investigating the social determinants of disease has traditionally been met with reluctance and resistance in health research:

one of the problems with social class is that we rarely study it in public health, rarely, even though everybody knows it's most important. The reason we in the United States don't study it is that first of all it's so overwhelmingly important that if you don't adjust for it statistically in your research, it overwhelms everything else under study and you'll never be able to study anything else. The second reason is you can't do anything about it anyway, they say. So why study a risk factor that you can't do anything about. ${ }^{249}$

However, considerable evidence has accumulated that the social determinants of health and disease are neither fixed nor immutable but are modifiable and that significant positive change is possible and practicable. Consequently, a fifth wave of public health development is currently emerging, seeking to investigate and address the systemic determinants of health and disease and driven by the convergence of multiple diverse perspectives and streams of evidence from inside and outside of medical healthcare.

Four major drivers of the emerging fifth wave of public health development are identified:

1. Amassed historical evidence demonstrating that biomedical healthcare is not a principal determinant of public health

2. Amassed evidence that social factors are the primary determinants of population health and disease

3. The emerging of community-led public health initiatives

4. The reframing of public health by the complexity sciences

\section{The Contribution of Medicine to Public Health}

Firstly, a vast array of historical, contemporary and international evidence ${ }^{206,226,228,229,250-252}$, accumulating since the mid-1970's, demonstrates that, contrary to orthodox belief, medical healthcare is not the principal determinant of improving public health. Associated with the demographic transition in global population and life expectancy, is an 'epidemiologic transition' as morbidity and mortality from infectious disease declined and the proportion of morbidity and mortality from non-communicable diseases such as heart disease, cancer and mental illness increased ${ }^{253}$. The orthodox narrative was (is) that advances in medical knowledge and practice were responsible for the decline in mortality rates from the 
serious and prevalent infectious diseases ${ }^{206}$, but examination of the historical record reveals a considerably different picture.

In the 300 years up to the mid-20 $0^{\text {th }}$ century - data from England and Wales reveals that mortality ${ }^{\mathbb{\phi}}$ due to many serious and prevalent infectious diseases including tuberculosis, pneumonia and measles had declined steeply prior to the introduction of effective medical treatments ${ }^{250}$. In the US, mortality data from the period 1900-1973, for 10 major infectious diseases (tuberculosis, scarlet fever, influenza, pneumonia, diptheria, whooping cough, measles, smallpox, typhoid and poliomyelitis) - which together accounted for $30 \%$ of total population mortality at the turn of the century reveal that mortality for nearly all diseases (with the exception of smallpox, for which effective treatment via vaccination was introduced in the early 1800's) dramatically reduced over the period, prior to the advent of effective medical treatment. Figure: the fall in standardised death rate (per 1000 people) for common infectious diseases in the USA between 1900-1973 in relation to the introduction of effective medical treatment. ${ }^{226}$
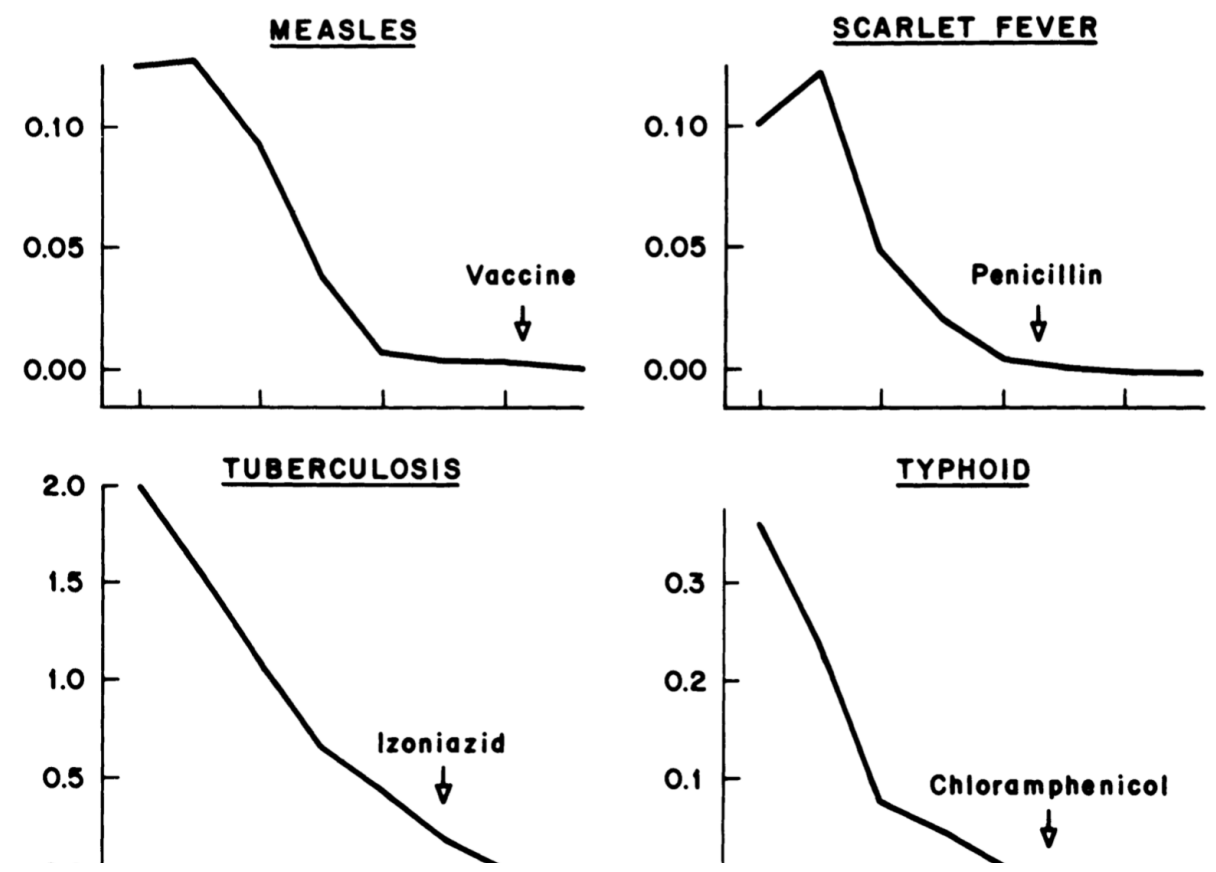

\section{TYPHOID}
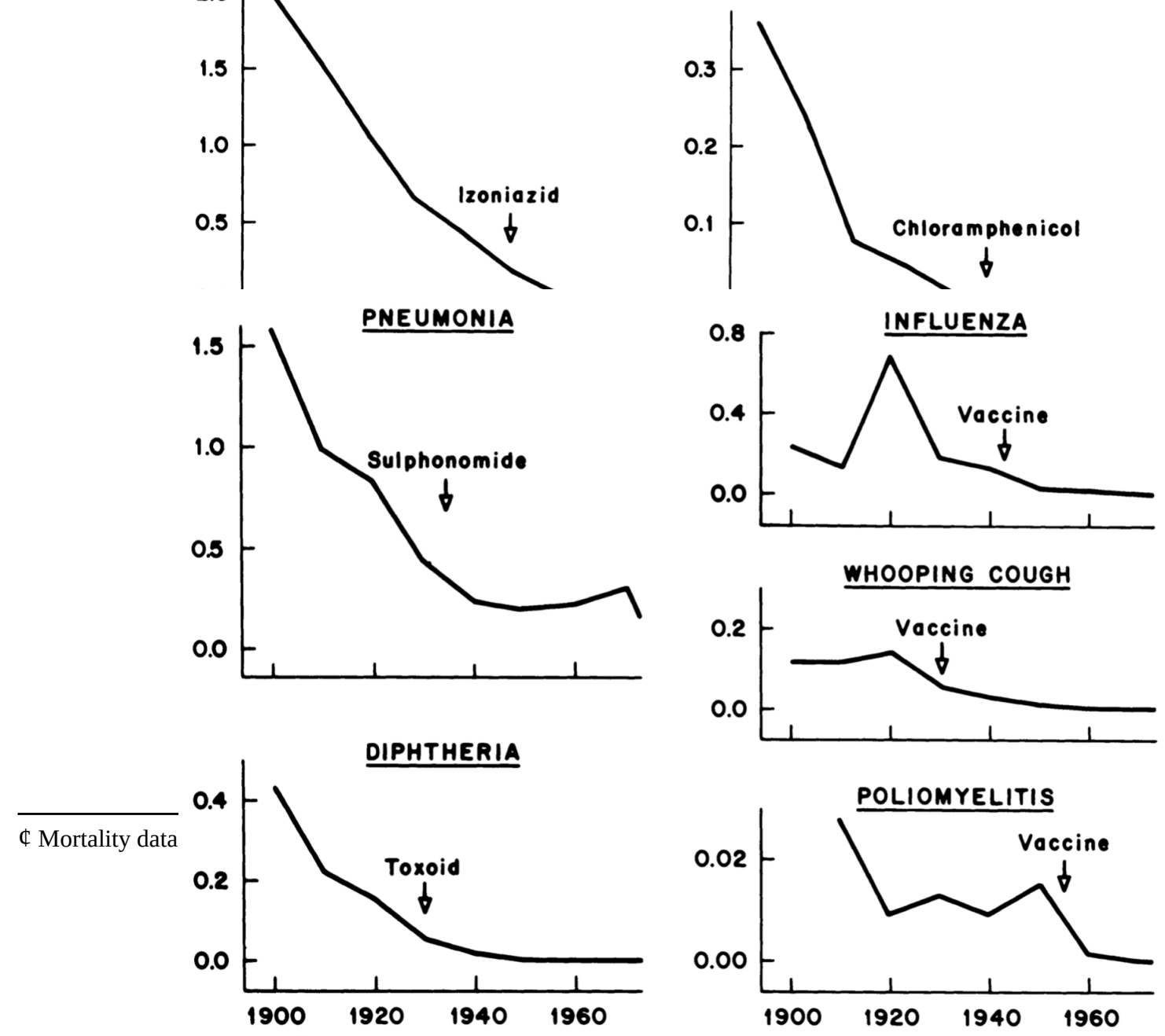
The total reduction in mortality attributable to the medical treatment of these diseases is estimated to be at most $3.5 \%{ }^{226}$. The contribution of biomedical healthcare to the improvement of population health, up to the mid-20th century, is thus relatively minor. In the contemporary period, international data for western industrialised countries from the mid-20th century to the present indicate that medical healthcare contributes between $15-25 \%{ }^{€}$ to public health ${ }^{257}$. Thus, even in terms of physical diseases, biomedical healthcare - while undeniably important - is not a principal determinant of population health.

The NHS [National Health Service] is an incredible institution, but it was not designed to create health and cannot do so on its own. The NHS spends almost all of its budget treating people after they become sick, and the majority of this expenditure is made in the last year of life. The idea that the NHS could improve health if only we gave it more money now forms a major obstacle to the real work of addressing the origins of [health disparities] and doing something effective about it. ${ }^{232}$

\section{Social Epidemiology}

The second driver of the fifth wave of public health development is the coming of age of social epidemiology - revealing that the principal determinants of health and disease are not reducible to individual biological and behavioural 'modifiable risk factors', but are the social factors - which are revealed in the features of neighbourhoods, communities, and local environments and that are outcomes of political, economic, cultural and historical forces. Thus, the fundamental causes of population health are social forces.

Many studies have described how it is not healthcare that influences health the most, but social and economic factors. The Marmot Review (2010), and other reviews of evidence, described that most of the drivers of ill health relate to social, economic, environmental, cultural and political factors that lie outside the immediate remit of the healthcare system. ${ }^{258}$

Unfortunately, a fixation on individual self-responsibility for changing modifiable risk factors has historically been associated with implicit and explicit beliefs that so-called 'preventable' or 'lifestyle' diseases such as heart disease, many forms of cancer and obesity are the outcomes of individual bad choices and bad behaviour thus effectively reframing disease as a signal of weakness, culpability and guilt. ${ }^{259}$

The ideology of ultimate individual self-responsibility for health and disease ignores what is known about the fetal and early-childhood determinants of adult

$€ \quad$ An earlier study by Bunker et al produced an estimated contribution of biomedical healthcare to public health of $43 \%$ - significantly greater than subsequent research. However this study was subsequently criticised for overly optimistic assumptions of efficacy and reliance on flawed evidence. ${ }^{254-256}$ 
disease $\mathrm{e}^{40,260}$ and how the social environment shapes and constrains opportunities, decision-making and behaviour ${ }^{261}$. For example, an early landmark study of more than 1800 adults aged 35 and over, in Alameda County in California, demonstrated that simply living in a high-poverty neighbourhood resulted in a $40 \%$ increased death rate from all causes, and a significantly increased death rate persisted even when traditional individual risk factors such as smoking, alcohol consumption, body-mass index, ethnicity, employment, personal income, access to medical care, marital status and psychological state were controlled for. ${ }^{262}$

The Alameda County study was one of the earliest studies to demonstrate that, independent of individual biology, choice and behaviour, an adverse 'sociophysical environment' - subject to processes such as disappearing social and cultural capital, declining public investment in infrastructure and services such as libraries and parks, declining private investment in businesses and collapsed life-opportunities - is a critical and independent driver of adverse health outcomes ${ }^{259}$. Yet, while debunking the ideology that places individual biology and behaviour at the centre of public health issues is a crucial step in advancing scientific and public understanding of the primary determinants of health, at the same time, there is a risk that by revealing the complexity of the problem and it's social, economic and historical roots, these issues may be deemed as lying outside the scope of healthcare and worse - as intractable.

\section{Organisation}

But, beginning in 1967, pioneering research shone an entirely new light on the social determinants of health and disease. The Whitehall studies of thousands of people in the British civil service demonstrated a stark social gradient in health even between affluent and well-resourced social classes - findings which have since been replicated throughout the developed industrialised world ${ }^{263}$, revealing specific and previously unrecognised social factors which are fundamental drivers of health and disease.

The first Whitehall study was longitudinal research carried out over 7.5 years that considered coronary heart disease mortality in more than 17,500 men in the British civil service, all of whom were 'white-collar' workers in stable employment. The second Whitehall study was of more than 10,000 female and male office workers in the British civil service, and began in 1985, continuing to this day ${ }^{264}$. The civil service workers were categorised into 4 grades which reflected their status, authority, rank and pay-grade: at the top are the senior administrators - the 'high-flyers', who tend to come from Oxford and Cambridge universities, who run the great departments of state, liaising with elected officials, making public policy and who tend to get knighted at the conclusion of their careers, next come the executives who implement government policies - including lawyers, economists, statisticians, doctors and scientists, then come the clerical workers who handle the paperwork and at the 
bottom is the 'other' grade consisting of 'unskilled' workers such as messengers and porters. ${ }^{263}$

The difference in mortality between the top and bottom social ranks was stark - even after controlling for (meaning eliminating their effects from analysis) known individual risk factors such as blood pressure, serum cholesterol, smoking, fibrinogen and social support - people in the bottom rank experienced 3 times more mortality than people in the top rank. But this was expected because social class is recognised in epidemiology as one of the most important predictors of health and disease ${ }^{249,265}$. What was totally unexpected was the finding that people in the $2^{\text {nd }}$ to top rank had about twice the mortality rate as people in the $1^{\text {st }}$ rank - part of a stepwise gradient in health and disease that traversed every level of the civil service. Furthermore, this gradient remained unchanged after controlling for the standard coronary heart disease risk factors, nor could the social gradient be explained by poverty or by lack of access to medical care - which was universal and free (in fact the data revealed that people in the lower ranks were more likely to receive investigation and treatment for heart disease). ${ }^{263}$

The people in the $2^{\text {nd }}$ to top rank such as doctors and lawyers were not suffering from poverty, poor nutrition, lack of education, substandard housing, poor health behaviours or inadequate medical care relative to people in the $1^{\text {st }}$ social rank - so all the usual suspects - the standard biomedical, psychosocial and socioeconomic risk factors couldn't explain the difference in mortality rates. Nor is the social rank gradient in disease confined to the British civil service - a similar stepwise gradient has been found in the wider society and in every society throughout the industrialised world for nearly every disease studied ${ }^{247,266}$ - indications of a major cause of preventable death that was going unrecognised and unaddressed in public health.

\section{Control}

For over a decade, researchers investigated several hundred factors including smoking, blood pressure, physical activity, job demands and social support, in an effort to explain the stepwise social gradient in all-cause mortality - but all the identified factors added together only accounted for about $25-33 \%$ of the gradient ${ }^{267,249,268}$. Then in 1997, researchers pinpointed the key factor driving the ubiquitous social gradient in morbidity and mortality as the degree of self-reported control that a person exercises in their environment. Control incorporates elements of agency and mastery - and can be described as a sense of self-efficacy deriving from a lived-experience of making effective decisions that drive meaningful consequences in one's life, including in one's work and lived environment. ${ }^{266,268}$

Accumulating evidence ${ }^{266,269}$ indicates that this sense of mastery and agency in one's life - the degree of control one experiences in the world is made, not born. 
"If I challenge you... [if you are a person from a relatively high socioeconomic class with the concomitant advantages of education and lived-experience]... with a very difficult life challenge about which you know nothing, I know that you're not worried about it, because I know what you're going to do: you're going to talk to some other people, you're going to make some phone calls, and within a day or two you're going to work out a scheme to deal with it. You may not know at the moment what's going to work, but you know that you'll be able to do it. That kind of confidence, and not only the confidence but the knowing how to go about solving the problem, is almost automatic. When I interview people in lower social class circumstances, and present them with that kind of problem, you can watch their shoulders slump with another life problem that they don't know how to deal with. It's not a question of intelligence, it's a question of knowing that you can work it out, and having the training and experience to work it out. Once you give people in lower social class circumstances a clue, they get it in a minute and they're off."249

In the workplace, the primary models driving the principles and practices of modern organisation have developed from lineages including industrial-era Scientific Management, Fordism and military centralised command-and-control ${ }^{270,271}$ producing social hierarchies with diminishing job-discretion and decision-latitude from top to bottom ranks, which - it is now evident - erode a person's sense of control, likely triggering biological responses that drive significant and severe health consequences along the gradient of power.

But low control is neither a natural nor inevitable feature of human organisation - a large body of historical and contemporary evidence demonstrates that, for example in so-called 'high-reliability organisations' operating in complex and dynamic environments such as commercial airline crews ${ }^{272}$, firefighting ${ }^{273}$, military ${ }^{274}$ surgical ${ }^{275}$ and software development ${ }^{276}$ teams, organisations have by necessity developed distributed rather than centralised command-and-control structures which rely on developing and leveraging individual agency, creative and adaptive capabilities in order to manage complexity.

The identification of work and life control as a primary driver of health and disease represents a turning point in scientific understanding of population health with critical implications for how we design and develop public health interventions. Investigating and modelling the mechanisms that translate social phenomena and processes such as work and life control into biological (health and disease) outcomes and developing interventions targeting those mechanisms are central to the development of the next wave of public health.

\section{Community-led Public Health}

The third driver of the fifth wave of public health development, is the reorienting of public health interventions from expert-led processes to community-led processes, in 
recognition that many expert-driven health-education interventions have proven to be ineffective ${ }^{247}$. Consequently, researchers are beginning to appreciate that the traditional research paradigm in which

"the outside researcher largely determines the questions asked, the tools employed, the interventions developed, and the kinds of results and outcomes documented and valued”277

may be driving the ineffectiveness of interventions. Research which is imposed in a hegemonic (top-down) way so that the community becomes the object of research manipulation - effectively reproducing larger historical patterns of social inequity - is likely part of the problem of public health development, because this approach attenuates community engagement by subordinating and silencing people's voices in what is done and why, ignores their priorities and substitutes compliance with the research goals for meaningful learning, development and change. ${ }^{278}$

In response, community-based participatory research methods have emerged which acknowledge, value and integrate both researchers' theoretical and methodological expertise and community members' real-world knowledge and lived-experience in a research framework that features ${ }^{277}$ :

- mutuality and joint participation

- co-design by researchers and community

- shared priorities and co-learning

- whole-systems development and community capacity development

- a focus on empowerment and increasing people's sense of control

- a balance between research and action

Describing this need to change how public health research and development is done, researchers argue that:

"if this nation is to transform society to eliminate health disparities and promote social justice, a more democratic and ecological approach to scientific study is necessary, one in which education between scientists and the public must take place in both directions. It is with this orientation to research, with its heavy accent on issues of trust, power, dialogue, community capacity building, and collaborative inquiry toward the goal of social change to improve community health outcomes and eliminate health disparities... [that emerging public health approaches are concerned with]"277 


\section{Complexity Science}

The fourth major driver of the fifth wave of public health development is the adoption of methods, frameworks, ideas and tools from the complexity sciences in public health research.

The failure to model the complexity of network dynamics in the system led to the death of forests under the supposedly sustainable practices of Scientific Forestry. A failure to model complexity, coupled with the imposing of simplistic 'solutions' upon complex problems is not a remote historical footnote but continues to be widely prevalent and evident in organisational responses to contemporary challenges and has led to the failure of interventions and adverse and disastrous systemic consequences in a broad range of domains including counterterrorism ${ }^{274}$, agriculture ${ }^{279}$, ecosystem management ${ }^{280}$, fisheries management ${ }^{281}$, social policy ${ }^{282}$, regulation of financial markets $^{283,284}$ and serious accident investigation ${ }^{285}$.

Two examples from the domains of biomedical healthcare and public health illustrate how the failure to model complexity can drive intervention failure and adverse consequences. In studies conducted at the Veterans Administration in the United States, military veterans suffering from substance addiction were evaluated 6 months post-rehabilitation treatment, with the findings that around $75 \%$ had relapsed and about $50 \%$ had returned to treatment ${ }^{286,287}$. According to one of the lead researchers:
"The context in which these vets went back to was dismal - insecure housing, insecure employment, insecure financial situation which ultimately led them back to their drug of choice - which was their primary coping mechanism and they relapse. That context was never part of the treatment plan, it was never part of all the models that were built to understand the lives of these individuals. And - this is where it becomes important - is then when those individuals came back they were penalised for that -“Why didn't you stay sober, why didn't you stay clean?” - because of a sort of reductionistic notion of what accounted for... the pathway that got them from "You're clean and sober" to "You're back to your addiction of choice".,"288

The second example is an analysis of the Healthy Summit 2010 Quality of Life Project $^{289}$ - an ambitious community-level version of the US Federal Government's Healthy People 2010 initiative which was a nationwide program of health promotion and disease prevention begun in 2000 and conducted over 10 years with 2 primary aims - to "increase quality and years of healthy life and eliminate health disparities" ${ }^{\text {290 }}$ in the US population.

In keeping with the nationwide Healthy People initiative, Summit County, a locality in the state of Ohio of approximately 1000 square kilometres, with a population of about 540,000 people predominantly distributed in metropolitan, town and village communities, established Healthy Summit 2010 in 2000 as a set of 20 wide-ranging goals for improving the economic, social, physical and behavioural health and 
wellbeing of the population over a 10 year project period through the coordinated and concerted efforts of local government councils, researchers, healthcare and social services organisations, healthcare providers, politicians, community leaders and activists $^{289}$. The goals included increasing the proportion of people living above the poverty line, reducing unemployment, reducing all-cause mortality, reducing domestic violence and increasing the coverage of prenatal care to pregnant women.

After 10 years of concerted multilevel effort, only 7 of the 20 progress indicators had improved (including teen birth rate and school attendance), while 7 had worsened (such as general poverty and unemployment) and the remainder had not changed. By way of comparison, at the national level, the Healthy People 2010 initiative had tracked health disparities (by ethnicity, gender, education, postcode, disability or sexual orientation) for 467 of its 969 objectives for improving population health (such as increasing people's physical activity, reducing the proportion of children and adults who are overweight and obese, decreasing cigarette and illicit drug use, increasing treatment coverage for people with mental illnesses and improving access to health care $^{262}$ ) but reported that after a decade health disparities had adversely increased for $13 \%$ of the objectives and had remained unchanged in about $80 \%$ of the objectives $^{291,292}$.

The recurrent failure of massive public health initiatives to resolve public health disparities has compelled researchers to seek beyond individual biological and behavioural factors to investigate what's happening (processes and forces) in the broader environment. Modelling of the determinants of public health is shifting from $4^{\text {th }}$ wave fixation on the individual, to $5^{\text {th }}$ wave situation of the individual in the stream of historical, social and economic forces that structure contemporary society, drive life-course trajectories and outcomes and produce different patterns of health and disease in diverse populations.

\title{
Investigating Health Disparities
}

\begin{abstract}
"When I was in my medical residency, I once admitted a six year-old boy to the hospital with his third serious exacerbation of asthma in less than two months. Each time that he was admitted and each time he went home on higher doses of medication. The medical team scratched their heads about what else could we be doing for this boy. His mom mentioned to me that they lived in a rundown apartment with lots of mold and I remember asking the senior attending physician whether there was any way to actually prescribe a new apartment. Unfortunately, the prescription pad really didn't work that way."293
\end{abstract}

A complex systems analysis of the Healthy Summit Project revealed that absent from the conceptual framework and design of public health initiatives and interventions is a modelling of the effects of 'place' - that certain neighbourhoods and communities have become sickness and poverty traps for generations of people, driving adverse 
life-course and disease outcomes. Neglecting this crucial upstream determinant coupled with the failure to model the historical, social and economic processes that create and shape contemporary neighbourhoods and communities ${ }^{294}$ - has led to the failure of well-resourced interventions to positively change downstream health and welfare outcomes of populations ${ }^{289}$.

The struggle of [people] against entrenched power is the struggle of memory over forgetting.

$$
\text { Milan Kundera }{ }^{295}
$$

Investigating the particulars of health disparities illustrates why public health research is beginning to reorient from a biomedical orthodoxy towards a more inclusive and diverse complex systems modelling of population health - as its understanding of causality and the congruence of interventions changes ${ }^{296}$. Health disparities exist across multiple dimensions including age, gender, class, postcode, sexual orientation and ethnicity. Individuals exist at the intersections of multiple dimensions of difference and the effects of social health inequalities in each dimension may not simply aggregate but interact - multiplying disadvantage $\mathrm{e}^{297}$. The following examination of African American population health is used to illustrate the complex causal relationships that drive disparities in health and disease and to introduce the distinction between systemic vs individual risk.

\section{Black and White}

Some of the starkest, widest, most studied and most persistent health disparities in the USA are between African Americans ('blacks') and European Americans ('whites') ${ }^{298,299}$. For example, infant and maternal health are regarded as significant markers of overall population health - African Americans have more than double the rates of infant mortality of European, Asian, Pacific Islander and Hispanic Americans and about 1.6 times that of American Indians. Maternal mortality (death related to adverse events in pregnancy) of African Americans is about 4 times higher than that of European Americans ${ }^{300}$. Of the leading causes of death, African Americans have more than double the rates of diabetes, kidney disease, septicaemia and hypertension compared to European Americans ${ }^{299}$.

Figure: US Maternal death rate per 100,000 live births ${ }^{301-303}$ 


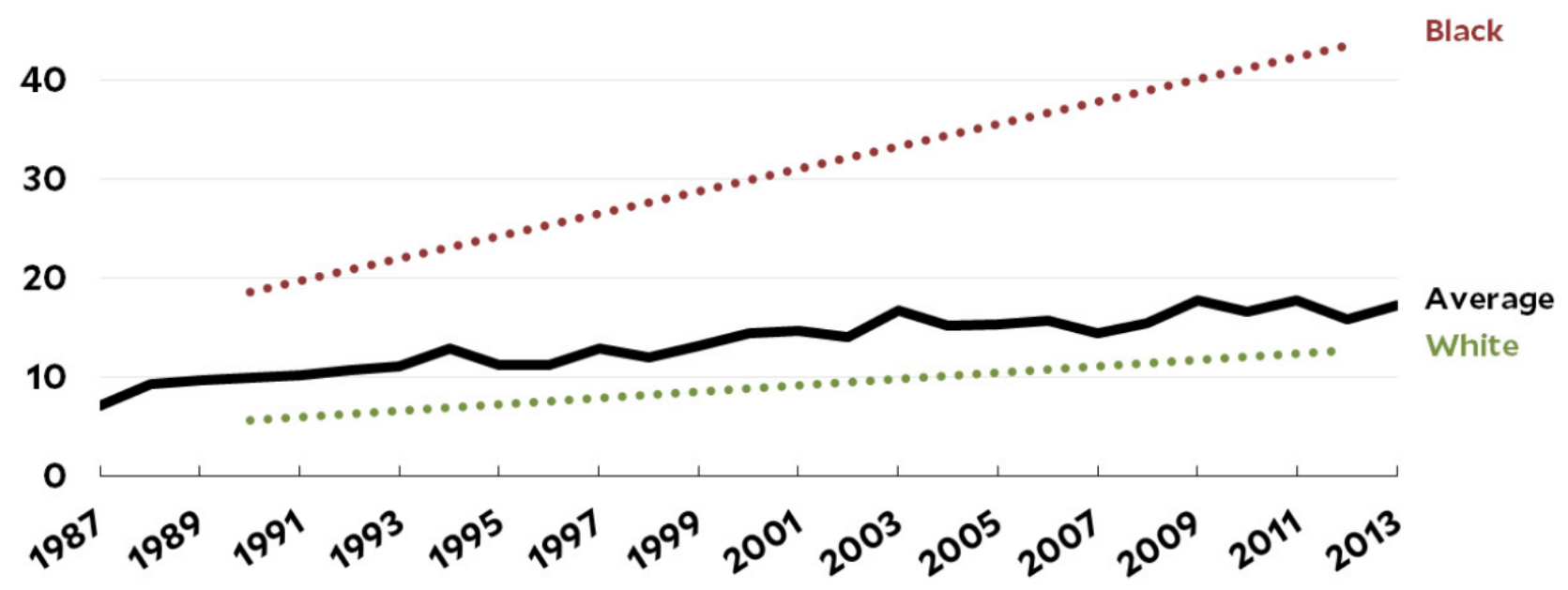

Genetic studies have failed to explain wide and persistent black-white health disparities ${ }^{304}$. For example, many studies have compared the birth and perinatal outcomes of recent foreign-born immigrants to the US compared to people of the same ethnicity born in the US - and have consistently demonstrated that the rate of low birthweight babies in recent migrants from African countries is comparable to that of US whites, but that in following generations, the birth outcomes of recent African migrants worsen and converge with that of US-born African Americans providing strong evidence for the operation of social rather than genetic determinants of health disparity ${ }^{305}$.

Figure: intergenerational worsening of birthweight in new African migrants to the US $S^{\text {ibid }}$

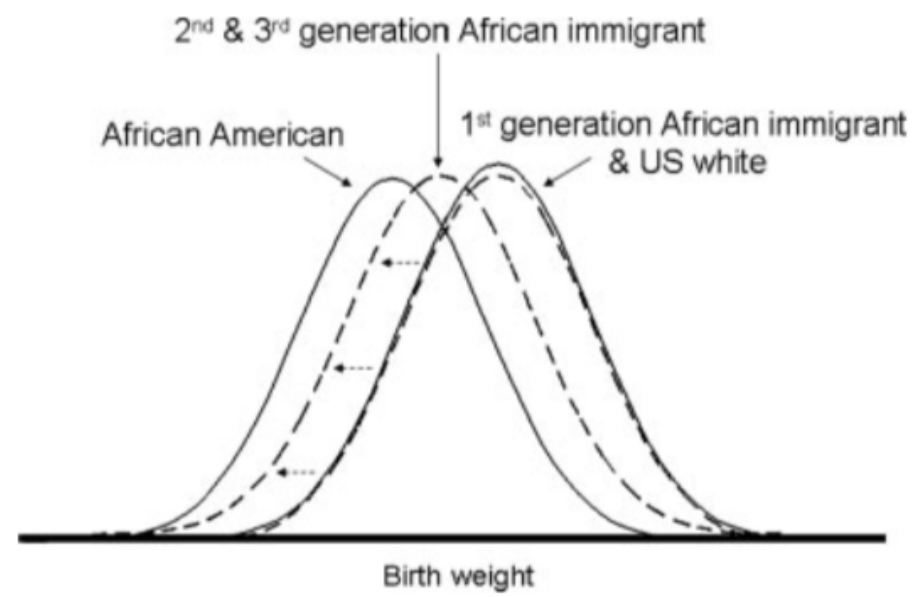

Historical and genetic evidence indicates that the ancestry of contemporary African Americans (as distinct from recent migrants from continental Africa) is an admixture of West African and European origins ${ }^{306}$. In the case of hypertension, where African Americans have 2.5 times the mortality rate compared to European Americans, research has demonstrated that the peoples of West Africa have lower rates of hypertension than European Americans and that for people of West African ancestry, rates of hypertension increase stepwise when measured across rural and urban African environments and in the Caribbean vs the United States - indicating that the 
primary drivers of the black-white health disparity are not genetic but epigenetic and social determinants ${ }^{307,308}$.

Black-white health disparities persist across age, gender, education and income ${ }^{307,309,310}$. Increasing income and education, which improve health outcomes in European Americans, largely fail to protect African Americans against health disparities $^{311-313}$. In fact, health disparities between blacks and whites tend to be bigger (in absolute and relative terms) at higher levels of education, for both men and women. For example a 23-25 year follow-up study of medical graduates revealed that African American physicians, compared to their European American peers, had significantly higher risk and earlier onset of cardiovascular disease, higher incidence rates of diabetes and hypertension (twice as high), higher incidence of coronary artery disease (1.4 times) and higher case fatality (52\% v 9\%) ${ }^{307}$. Blacks with a college (tertiary) degree or higher have lower life expectancy than whites and Hispanics who only completed high school ${ }^{314}$.

Public health approaches to 'closing the gap' of black-white health disparities have classically adopted 3 strategies ${ }^{315}$ :

1.Behavioural - for example attempting to get people to change their diet and exercise more

2.Biomedical - improving supply and access to healthcare services

3.Socioeconomic - policies and programs to improve educational and employment outcomes

The failure of these $3^{\text {rd }}$ and $4^{\text {th }}$ wave public health strategies - which classically model 'problematic' individuals and populations abstracted from place, history and other dimensions of lived context - to reduce persistent health disparities is driving the investigation and development of more systemic or 'complex systems' models for improving public health.

"The entrenchment and, in some cases, worsening of black health disadvantages occurred across a wide range of health outcomes and during a period when the reduction or elimination of health disparities was identified as a high-priority national health objective. The clear failure to meet this objective suggests that future success may require new conceptual models and deepening understandings of the sources and mechanisms leading to health disparities."316

\section{Hypersegregation}

"In many U.S. cities, life expectancy can vary by as much as 25 years across neighborhoods. The same dramatic geographic disparities can be seen for other outcomes, such as infant mortality, obesity, and the prevalence of diabetes and other chronic diseases.”317 
The explanatory failure of $4^{\text {th }}$ wave public health modelling has compelled researchers to investigate upstream social, economic and historical factors to understand and model persistent and widening ethnic health disparities. Amassing evidence indicates that pervasive segregation in education, jobs and communities coupled with massive wealth inequalities and systemic racism are primary determinants of the health disparities between African Americans and European Americans $^{318}$.

"blacks currently live under a level of segregation that is higher than that of any other immigrant group in U.S. history"319

Systemic and historical differentials of exposure to adversity and opportunity have created the contemporary health, wealth and social disparities between African Americans and European Americans, which have become normalised as everyday features of the cultural landscape. Most people currently living in the United States have grown up and/or reside in ethnically and economically segregated neighbourhoods $\mathrm{s}^{320}$. Both education and the job market are segregated by ethnicity, place (neighbourhood) and class ${ }^{309}$. But these structures and features of inequality and disparity are neither natural nor accidental.

Residential ethnic segregation is measured in 5 dimensions:

1. Unevenness is the degree to which ethnic groups are unevenly distributed across neighbourhoods in a metropolitan area

2. Isolation is the extent to which people live in neighbourhoods that are predominantly made up of their own ethnic group separate from others

3. Clustering is the degree of closeness or proximity of neighbourhoods made up of predominantly one ethnicity

4. Concentration is the relative amount of physical space occupied by an ethnic group within a given metropolitan environment

5. Centralisation is the proximity of the neighbourhood to the central city (ie inner city)

Hypersegregation is defined as ethnic residential segregation occurring in at least 4 of the 5 dimensions of segregation simultaneously and is a critical marker of social inequality. Despite ethnic segregation decreasing since the 1970's, more than half of all African Americans currently living in metropolitan areas live in areas of high to hypersegregation ${ }^{321}$. 


\section{Suburbs and Ghettos}

Figure: Twitter Aug.2019322

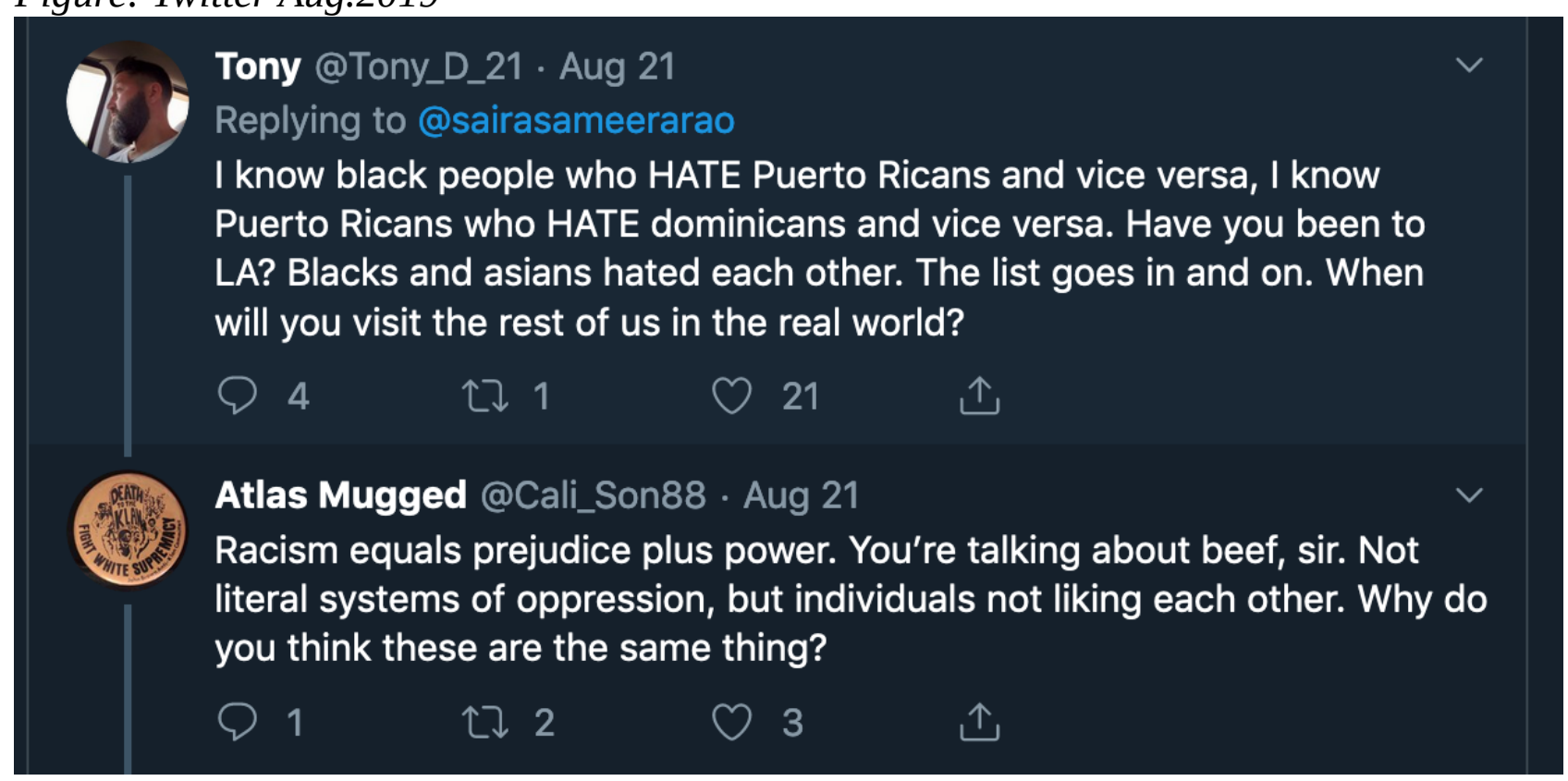

"I think white people are sick to death of hearing about black grievances, and part of that comes out of an ignorance of history and a reluctance to face up to that part of our history, which is an indigestible lump." 323

Massive and ubiquitous residential segregation is not an historical accident nor a product of spontaneous ethnic clustering but an outcome of intentional social engineering - interlinked federal, municipal and institutional policies and practices that were designed and developed to systematically build advantage in white households while disadvantaging and disenfranchising black households.

For example, from 1924 to 1950 the Code of Ethics of the National Association of Real Estate Boards required that

"A realtor should never be instrumental in introducing into a neighborhood... members of any race or nationality...whose presence will clearly be detrimental to property values in the neighborhood." ${ }^{324}$

In the early $20^{\text {th }}$ century, Anglo-American prejudice and discrimination against African Americans became enshrined and extended in government policies. The federal government established the Home Owners Loan Corporation which created the modern mortgage in 1933, and the Federal Housing Administration which administered housing insurance in 1934. These agencies produced standardised appraisal methods for rating neighbourhood value and appeal containing explicit ethnic criteria according to which homogeneously white neighbourhoods were bestowed the highest value while even one African American family in residence 
could earn an entire neighbourhood the lowest mortgage eligibility rating regardless of the quality of its housing stock.

The agencies made their maps and classifications of 'risky' and 'safe' neighbourhoods available to private lending firms which adopted the government's standards en masse, thereby nationally systematising discrimination and driving the residential ethnic segregation that characterises contemporary American society ${ }^{325,326}$. Data from the FHA itself contradicted it's own assertion that the presence of African Americans lowered property values - in fact integration tended to increase property values because working and middle-class African American families seeking to escape the inner cities and facing restricted options, were often willing to pay much more than 'fair market value' to obtain residential property ${ }^{327}$.

Following WWII, a massive state-sponsored exodus of white working and middle class families from the cities into the suburbs began. In 1950, about $65 \%$ of the urbanised population lived in central city districts, with the remaining 35\% residing in the suburbs. By 1990, the ratio had flipped, with 65\% residing in rapidly expanding suburban areas ${ }^{277}$.

The state's social engineering of 'white flight' from the cities to the suburbs is exemplified in the history of Levittown - a massive housing development of 17,500 homes, built between 1947-1951, in response to the housing needs of returning war veterans and their families. The houses were constructed using an innovative 'reverse assembly-line' process of specialised crews performing piecemeal construction tasks along a line of allotments so that an entire house was built in a day ${ }^{278}$, and 150 houses built per week. The houses were sold for about $\$ 8000$ (approximately $\$ 90,000$ in current dollars ${ }^{279}$ ) with no downpayment required. For many buyers, monthly mortgage repayments were less than what they'd previously been paying in rent in public housing. The federal government underwrote almost the entire cost of the project having obtained congressional approval to guarantee bank loans for largescale suburban development. By 1948, most suburban housing development nationwide was using this government financing - enabling developers to build neighbourhoods even before finding buyers, such was the demand $\mathrm{d}^{273}$.

In order to obtain government financing, developers submitted their plans to the Federal Housing Administration for evaluation which considered factors such as construction materials, design specifications, neighbourhood zoning and sale price. Federal agency approval enabled developers to negotiate low-interest bank loans to finance the acquisition of land and construction and government guaranteed insurance enabled the banks to issue loans to home buyers with minimal risk. Approval was also conditional upon developers making a commitment not to sell homes to African Americans and was even withheld if African Americans lived in nearby neighbourhoods due to the perceived risks of desegregation. Thus, in the postwar decades, the nation was suburbanised and ethnically segregated by federal and 
institutional policies and practices ${ }^{273}$, that engineered 'white flight' into the suburbs and drove blacks into increasingly impoverished inner city ghettos.

\section{The Social Engineering of Inequality}

The advantages that federal housing programs and policies conferred on millions of white families in the 1940s and 1950s, and which were denied to otherwise equally eligible working and middle class black families, produced massive and widening wealth inequalities within contemporary American society by enabling the massive accumulation of housing equity - the principal component of wealth accumulation in the working and middle classes - in white households as house prices grew by on average 43\% from 1973-1980, while wages stagnated. By the time the Fair Housing Act was passed in 1968, prohibiting future discrimination - housing unaffordability and the absence of reparative justice served to keep black homebuyers out of white suburbs.

Classically, household income (from wages or from capital gains) has been a commonly identified metric of socioeconomic inequality. In the US, the median household income of European American families is currently about $\$ 60,000$, while median African American family income is about $60 \%$ of that, approximately $\$ 37,000$. But it has become apparent that it is actually household wealth (assets minus debts) that more accurately reveals the nature of inequality. Median white household wealth is currently about $\$ 134,000$, while median black household wealth is less than $10 \%$ of that, approximately $\$ 11,000$. If depreciating assets such as automobiles, electronics and furniture are taken out of the equation, median wealth for black households drops to $\$ 1,700$ compared to $\$ 116,800$ for white households - that is median black household wealth is only $1.5 \%$ of median white household wealth. Furthermore, the median household wealth of African American and Hispanic American households is falling and on a path to zero by 2053 and 2073 respectively, while European American median household wealth continues to increase ${ }^{328}$.

\section{Sickness and Poverty Traps}

The contemporary social and economic stratification of American society by colour, the pervasive contemporary segregation of neighbourhoods and communities and the massive contemporary wealth disparity between Anglo-European, African and Latino/Hispanic American households - are direct outcomes of a broad array of federal and municipal policies and institutional practices discriminating against, disadvantaging and disenfranchising African Americans - enacted in domains including public housing development, municipal zoning, highway construction (fencing in and fencing out of poor neighbourhoods), environmental zoning that places hazardous industries in disadvantaged neighbourhoods, suburban development and public and private disinvestment from disadvantaged neighbourhoods ${ }^{327}$. 
The association between family and neighborhood factors and the risk of violent crime is identical for blacks and whites. However, because of residential segregation, blacks are more exposed to these conditions than whites. In the 171 largest U.S. cities, there is not even one in which whites live in socioeconomic conditions that are comparable to those of blacks...The worst urban context in which whites reside is considerably better than the average context of black communities. $^{329}$

The pervasive and persistent discrimination that characterises the lived experience of African Americans originates in 244 years of slavery (1640-1863) during which at least 8 million African people were captured and transported to America to be sold and enslaved, with many millions more dying en route. The institution of slavery was brought to an end by the American Civil War (1861-1865) fought over the threat of secession of slave-owning Southern states ${ }^{330}$ and was followed by the era of Reconstruction and Jim Crow (apartheid) laws over the next 102 years (1863-1965) which mandated segregation in public schools, public places, public transportation, restaurants, drinking fountains and toilets and which in turn compelled the struggle for Civil Rights arguably extending from 1965 into the present ${ }^{300}$. Historic enslavement and exploitation, systemic discrimination throughout the life-course, outright violence, sanctioned terrorism and myriad forms of micro and macroaggression and prejudice by whites against blacks created a template for contemporary social stratification and ethnic relations in the USA ${ }^{325,327,331,332}$.

For more than two centuries, enslaved African Americans labored arduously (usually on land stolen from Native Americans) to develop agricultural and other economic prosperity for millions of white Americans in many walks of life. For many white families, this early prosperity led to some assets being passed down over later generations of whites to the present day. This wealth generation [continues to be visible] in many areas. For example, the trade in enslaved Africans and African Americans was a central reason why New York City early became one of the world's major cities... Enslaved workers also constructed the great buildings that have become the most important political symbols of the United States - the White House and the Capitol in Washington, D.C. Ironically, these enslaved black workers put a bold Statue of Freedom on the top of that Capitol dome. ${ }^{290}$

Nearly all American history textbooks used in schools across the country omit or even outrightly deny the federal government policies that have produced the pervasive residential segregation of contemporary US neighbourhoods and the economic disenfranchisement of the African American population revealed by household wealth disparity ${ }^{327}$. The widespread dissemination of a 'whitewashed' history of how contemporary society is socially and economically structured and the consequent collective ignorance and forgetting of actual history have been identified as perhaps the most critical sociocultural (or 'collective consciousness') barriers to reparative justice for addressing black-white systemic inequality in the US ${ }^{333}$. 
Although the majority of poor persons in the U.S. are white, poor white families are not concentrated in contexts of economic and social disadvantage and with the absence of an infrastructure that promotes opportunity in the ways that poor blacks and Latinos are. The neighborhoods where minority children live have lower income, education and home ownership rates and higher rates of poverty and unemployment compared to those where white children reside. In fact, in 100 of America's largest metropolitan areas, 75\% of all African American children and $69 \%$ of all Latino children are growing up in more negative residential environments than the worst off white children. ${ }^{299}$

Thus a constellation of connected and interacting factors reaching from the past and continuing into the present including ${ }^{334}$ :

- residential hypersegregation

- massive and widening wealth inequity

- systemic discrimination

- political disenfranchisement

- concentration of disadvantage

- public and private disinvestment

- few employment and economic opportunities due to suburban flight of middle and high socioeconomic classes

- lower quality schools

- substandard housing

- exposure to toxicity and pollution from nearby industry

- lack of municipal investment in public transport and urban infrastructure

- lack of health and social services

- lack of amenities such as parks and libraries

- prevalent crime and drug abuse

- policing that's experienced less as 'protecting and serving' the community and more like an occupational force

- a retributive judicial system

- lack of markets with fresh food

- prevalence of alcohol and fast food outlets

have created sickness and poverty traps in inner city neighbourhoods across the USA for generations of people, driving demoralisation, thwarted development, collapsed life chances, social defeat and adverse health outcomes. ${ }^{\ddagger}$

¥ Exemplified in the case of Detroit - with population exposure to multiple adversities including macroeconomic destabilisation - loss of manufacturing jobs at end of the $20^{\text {th }}$ century, public and private disinvestment, flight of middle and high socio-economic status whites, collapse of municipal authority, a state-enforced austerity regime, defunding of public infrastructure and services and escalating poverty ${ }^{335}$ 


\section{Systemic Risk}

Despite growing interest in understanding how social factors drive poor health outcomes, many academics, policy makers, scientists, elected officials, journalists, and others responsible for defining and responding to the public discourse remain reluctant to identify racism as a root cause of racial health inequities. ${ }^{320}$

The term 'systemic risk' is proposed to encompass the environmental carriers including political, historical and economic drivers of adverse population health outcomes and health disparities, that create the social topography through which individual biological and behavioural risks emerge and are situated ${ }^{296}$.

The pattern of ethnic health disparities observed in the US are mirrored in other post-colonial countries including Australia, Brazil, New Zealand, Britain and South Africa in which economic and political advantages drive better health outcomes in a socially dominant ethnic group(s) compared to unadvantaged and disadvantaged ethnic groups - indicating that systemic risks supervene national, cultural and genetic contexts ${ }^{299}$.

The construct of systemic risk begins to frame how disadvantage, inequity and adversity can become concentrated and persistent within places and populations, creating a 'risk-environment' shaping individual life-course trajectories and outcomes and how, in the absence of reparative justice (meaning collective sense-making and social restitution ${ }^{336}$ ) the continuity of systemic risks enables poverty and sickness to be transmitted across generations.

\section{Racism as a Systemic Risk Factor}

"I asked a retired printer from New York City how often he had faced discrimination over his eight decades of life. After reflection, he estimated that he confronts at least 250 significant incidents of discrimination from whites each year, if he only includes the incidents he consciously notices. Judging from my field studies and those of my students that have used in-depth interviews with African Americans, this man's experience seems representative. Over the course of a typical lifetime, a black man or woman likely faces thousands of instances of blatant, covert, or subtle discrimination at the hands of whites." 337

Evidence is amassing that racism and other forms of discrimination are critical and primary causes of public health disparities. Racism and ethnic discrimination produce earlier onset, higher rates and greater severity of illness and impairment and higher rates of death in the US and globally ${ }^{307}$. An extensive systematic review revealed that racism is associated with poorer mental health (including conditions such as depression and anxiety), poorer physical health and poorer general health and that the effects of racism on health were not moderated by age, gender, birthplace or education ${ }^{338}$. Systematic reviews reveal 
that African American residential segregation - a central feature of racism in the US - is associated with increased risks of low-birth weight, pre-term birth and stillbirth, increased exposure to known cancer risks, delayed diagnosis and is increasingly implicated in lower survival rates from a broad range of cancers including breast, lung, prostate, colorectal, cervical, liver and ovarian ${ }^{314,339}$.

In the United States, racist thought, emotion, and action are structured into the rhythms of everyday life. They are lived, concrete, advantageous for whites, and painful for those who are not white. Each major part of the life of a white person or a person of color is shaped directly or indirectly by this country's systemic racism. Even a person's birth and parents are often shaped by racism, since mate selection is limited by racist pressures against intimate interracial relationships and intermarriage. Where one lives is frequently determined by the racist practices of landlords, bankers, and others in the real-estate profession. The clothes one wears and what one has to eat are affected by access to resources that varies by position in the U.S. racial hierarchy. When one goes off to school, one's education is shaped by contemporary racism-from the composition of the student body to the character of the curriculum. Where one goes to church is often shaped by racism, and it is likely that racism affects who one's political representatives are. Even getting sick, dying, and being buried may be influenced by systemic racism. Every part of the life cycle, and most aspects of one's life, are shaped by the racism that is integral to the foundation and continuing operation of the United States. ${ }^{337}$

Research is demonstrating the effects of racism throughout the life course and across generations - for example how fetal and childhood exposures to adversities drive later onset adult disease such as diabetes and cardiovascular disease, and how the cumulative effects of exposure to racism across multiple domains such as at work, at school or college, in public environments such as shopping centres and on the street or while seeking healthcare - produce a 'dose-response' (meaning escalating) association with adverse health outcomes $^{340,341}$. In Australia, Indigenous children are commonly exposed to racism, often in the first years of life ${ }^{342}$. A recent longitudinal study assessing the health outcomes of 1239 Indigenous children aged 5-10 who were tracked for 6 years, demonstrated significant association between exposure to direct and vicarious racism and a broad range of adverse mental and physical health indicators. Vicarious trauma was defined as the child's caregiver's livedexperience of recurrent racism and discrimination and was associated with an increase of 1.7-2.6 times greater risk of mental health issues, sleep difficulty and asthma in the child - illustrating the operation of systemic risk ${ }^{343}$.

Blacks must be constantly aware of the repertoire of possible responses to chronic burdensome discrimination. One older respondent spoke of having to put on her "shield" just before she leaves the house each morning... she said that for more than six decades, as she leaves her home, she has tried to be prepared for insults and discrimination in public places, even if nothing happens that day. ${ }^{344}$ 
Racism as a validated biological 'toxin' is a complex phenomenon - having institutional, historical, economic, political, cultural and interpersonal dimensions. Furthermore, racism 'evolves' - changing its expression and features in time and place and can be covert and coded or unconscious ${ }^{345}$. As researchers seek to measure the health impacts of racism, this complexity creates a conceptual challenge - how can racism itself be measured? ${ }^{346,347}$ One innovative proxy measure of racism - is 'big data' amassed from millions of independent searches using the internet search engine Google, which can be filtered and analysed by geographic location. What we type into search engines tends to reflect more of our unfiltered and uncensored personas than what we may reveal in public - and which may not be reliably captured in a survey of self-reported discriminatory attitudes. ${ }^{348}$

Search engine queries have been demonstrated to explain a large part of the variation between different geographical areas in terms of voting, religion and gun ownership (for example by search volume for phrases including terms such as 'God' and 'guns') - indicating that search engine data is strongly correlated to population-level characteristics. Researchers obtained data from internet searches for the ' $n$-word' for 196 areas across the USA encompassing about 99\% of all eligible voters to assess what they called 'area racism'. Racist epithets and derogatory phrases are highly searched terms in the US ${ }^{348}$. The researchers demonstrated a dose-response relationship between area racism towards African Americans and adverse health outcomes in the African American population. For every increase of one standard deviation in the level of racism revealed by internet search volume, there was a $5 \%$ increase in the prevalence of preterm birth, a $5 \%$ increase in the prevalence of low-birth weight newborns and between 3.6-8.2\% increase in all-cause mortality across all ages ${ }^{349,350}$.

\section{How Systemic Risks Become Biological Processes}

Determining how structurally rooted social processes work through biological mechanisms to impact health is fundamental to understanding racial, ethnic, and socioeconomic health inequality. ${ }^{335}$

The experience of the systemic discrimination that African Americans experience over the life-course has been compared to the game Jenga - in which players build a tower from wooden blocks, then take turns removing blocks from anywhere in the structure and stacking them on top of it - making the tower progressively more unstable, the game being lost by the player whose move topples the tower.

"They pull out one piece at a time, at a time, and another piece and another piece, until you sort of collapse... you start losing pieces of your health and well-being, but you still try to go on as long as you can. Even if you're disabled, even if it's hard, that you have a certain tenacity and hope, and sense of collective 
responsibility whether that's for your family or community. But there's a point where enough pieces have been pulled out of you, that you can no longer withstand, and you collapse." 351

\section{Catastrophising Teen Pregnancy}

Research in the field of teen pregnancy has pioneered the exploration of how systemic risks such as pervasive racism, discrimination and inequality become translated into sickness and adverse health outcomes; unravelling and identifying biological pathways and mechanisms through which lived experience becomes biologically 'embedded' and 'embodied' ${ }^{352}$.

Considerable differences in rates of teen pregnancy are found both between and within countries. ${ }^{353}$

Figure: Teen pregnancies per 1000 women aged 16-19

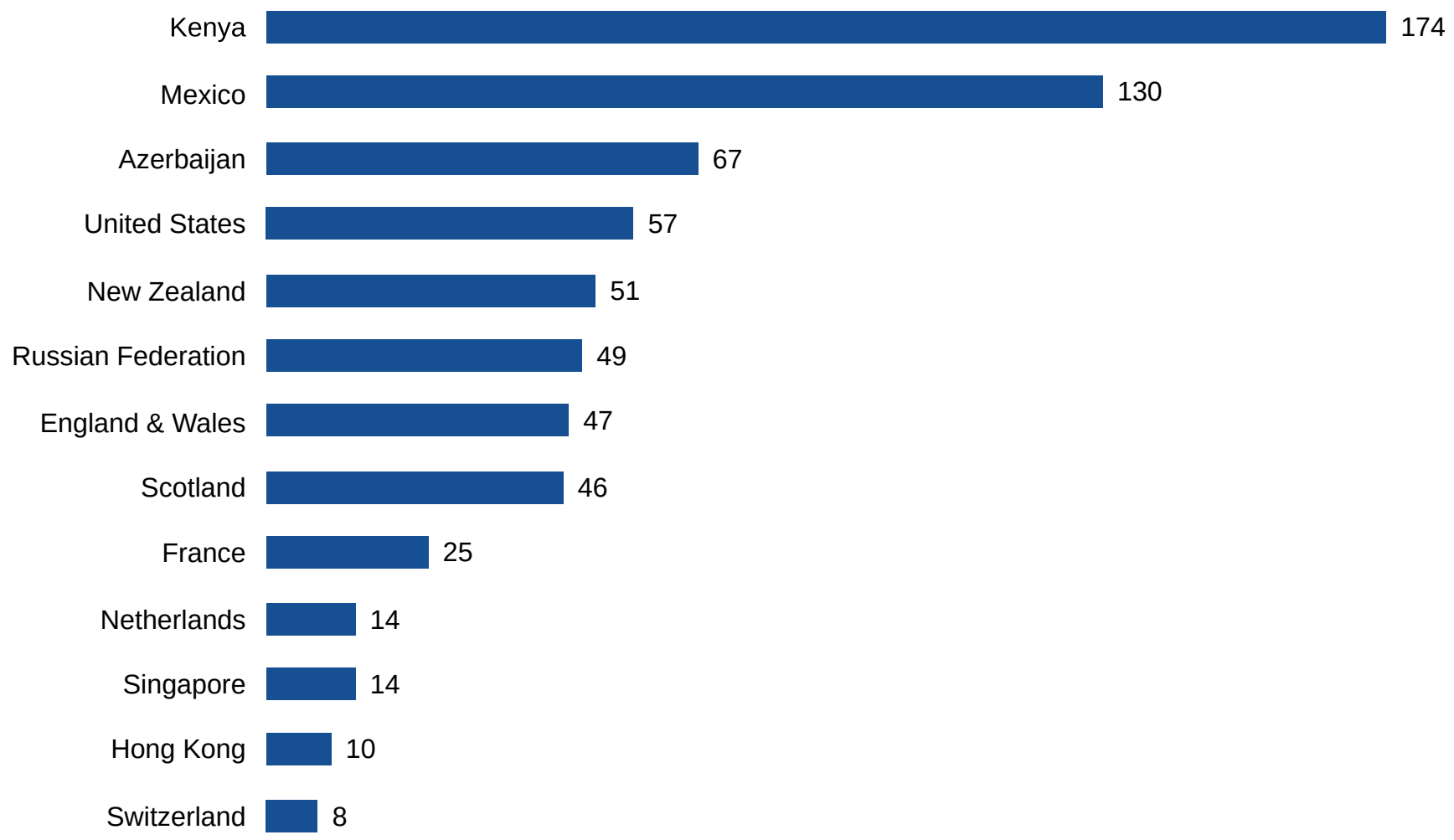

The US and UK have some of the highest rates of teen pregnancy amongst western industrialised countries. Within countries, teenage pregnancy is strongly associated with inequality and adversity - with significantly higher rates amongst the most socioeconomically deprived groups. ${ }^{353,354}$

The December $9^{\text {th }}, 1985$ cover of Time magazine featured a pregnant white teen, and a feature story titled "Children Having Children: Teen Pregnancy in America” with the subtitle "Teen pregnancies are corroding America's social 
fabric.”355. By 1995, in his State of the Union Address, President Bill Clinton had identified teenage pregnancy as “our most serious social problem”. Back in 1968, a leading demographer had written a dire prediction that became widely quoted:

The girl who has an illegitimate child at the age of 16 suddenly has 90 percent of her life's script written for her. She will probably drop out of school; even if someone else in her family helps to take care of the baby, she will probably not be able to find a steady job that pays enough to provide for herself and her child; she may feel impelled to marry someone she might not otherwise have chosen. Her life choices are few, and most of them are bad. ${ }^{356}$

Around the late 1960's to early 1970's, a remarkable inversion of cultural logic occurred in the US, that became widely articulated in political, academic and media discourse - teen pregnancy went from being characterised as just another consequence of poverty and inequality, to becoming nominated as a main cause of poverty and inequality 35,356 . The reproductive behaviour and fertility patterns of African American women became a particular target for racialised public anxiety:

Unless someone or something intervenes during [this] little girl's life, there is good reason to believe that [she] will be condemned to repeat the same vicious cycle that took hold of her mother, her grandmother and her great-grandmother, by having a baby during - or perhaps even before - her teens. The striking number of teenage girls having babies is a priority health concern in Chicago and across the nation, for the children born to these mothers are often premature and growth retarded and can suffer a number of devastating complications at or shortly after birth that can compromise the quality of their life. But the impact of adolescents having babies has consequences far beyond the medical ones in communities of the black underclass. Once the cycle takes hold and a young girl has a child, her ability to improve her economic or social condition and pull herself out into a more productive segment of society is limited. Often, she drops out of school, has no job skills and falls into the welfare trap for support. In monetary terms, [she] has already been an expensive baby. The price of the heroic medical efforts employed to salvage her life had surpassed $\$ 120,000$ by the time she was released from the hospital... As she and thousands of youngsters like her grow up in environments that have little to offer in the way of nurturing, educating and inspiring, there can only be additional costs. ${ }^{358}$

\section{Fairytales and Statecraft}

Historical analysis reveals that the public discourse about teenage pregnancy in the US represents a moral panic that is largely unsupported by the facts. One of the most prominent authorities in the field, reviewing 4 decades of evidence, observes that orthodox narratives about teenage pregnancy (articulated in academic, political, media and popular discourse) are based on systematic 
distortion, misunderstanding and misrepresentation of inequality, poverty, gender and ethnicity - which serve to divert public attention from the systemic causes of inequality and simultaneously blame disadvantaged people for their adverse life-course outcomes.

In fairy tales, there are two possible outcomes for a young girl. In the Disney version, the handsome prince rescues her, then marries her, and everyone lives happily ever after. In the dark version, the heroine makes a dreadful mistake that leads to disaster. For the past 15 years, political pundits have been telling us a dark fairy tale about American teens, blaming [those] who have babies out of wedlock. This assumption guided the welfare reform act of 1996, which promised to write America a happy ending by getting teens to stop having babies, get married, and thus end poverty. But [longitudinal research] shows that fairy tales have no place in the realm of policy-making [and] that teen childbearing is NOT the reason that many Americans have been trapped in poverty over the past three decades. ${ }^{359}$

Notably, the stoking of public concern, moral panic and hostility towards the problematised 'other' in order to drive a political agenda is an age-old strategy of statecraft (realpolitik) and has been described previously in the inception of Scientific Forestry in $18^{\text {th }}$ century Germany and in the hegemony of Cognitive Behavioural Therapy in the $20^{\text {th }}$ century in the UK.

By the mid-1980s, Congress had created a new federal office on adolescent pregnancy and parenting; 23 states had set up task forces; the media had published over 200 articles, including cover stories in both Time and Newsweek; American philanthropy had moved teen pregnancy into a high priority funding item; and a 1985 Harris poll showed that 80 percent of Americans thought teen pregnancy was a "serious problem" facing the nation, a concern shared across racial, geographic, and economic boundaries. ${ }^{360}$

A landmark longitudinal study of over 300 teen mothers and their children, most of whom were disadvantaged and many of whom were African American, whose life course trajectories and outcomes were tracked for 30 years beginning in the mid-1960s, demonstrated that teenage childbearing is not a 'root-cause' of social disadvantage, did not trap young mothers in lifelong welfare dependency and had only a modest effect on their educational and economic achievements later in life relative to otherwise similar women who delayed childbirth until their twenties. At 17 years follow-up, many of the teen mothers had returned to school, were not on welfare payments, had found stable employment and $1 / 4 \mathrm{had}$ achieved middle-class incomes ${ }^{361}$. Overall, more than $3 / 4$ of the teen mothers went on to graduate from high school and 1 in 10 achieved a college degree very similar outcomes compared to women in the same disadvantaged demographic cohort who hadn't gotten pregnant as teenagers. Most of the women (62\%) had not had large families - having at most 1 further child, and only a small minority had had more than 3 children, counter to the oft-touted 
stereotype of the 'welfare queen'. Nor were their offspring condemned to bad outcomes with the majority faring reasonably well at the 30-year follow up ${ }^{362,359}$.

current research no longer supports the notion that teenage childbearing is a devastating event. ${ }^{363}$

Cumulative research demonstrates that educational outcomes of the children of teen mothers is on par or even superior to that of their peers born to otherwise similar older mothers, and furthermore that the estimations of the economic burden to society attributed to teenage pregnancy have been greatly exaggerated. ${ }^{364}$ A recent longitudinal study of young Australian women aged between 18-23 found no evidence of an adverse impact of younger maternal age on education, employment or income. ${ }^{365}$

Early childbearing was relatively common amongst European settlers in colonial-era America. In general, the timing of family formation is coupled to the social environment including economic opportunity. In the agricultural era, when farmland was abundant, European settlers began childbearing early and had large families. The timing of childbearing and marriage changed as the nation transitioned from an agrarian to an industrialised economy - decreasing in periods of economic growth and increasing in periods of decline. The Great Depression curtailed fertility rates in older and younger women. In the postWWII economic expansion, fertility rates for all ages soared, particularly in the decade between 1955-1965. Public angst about the supposed epidemic of teen pregnancy was peaking decades after the actual rates of teen pregnancy had sharply declined. ${ }^{356}$

Figure: Birthrates Among American Women by Age, 1955 to 2004 ibid

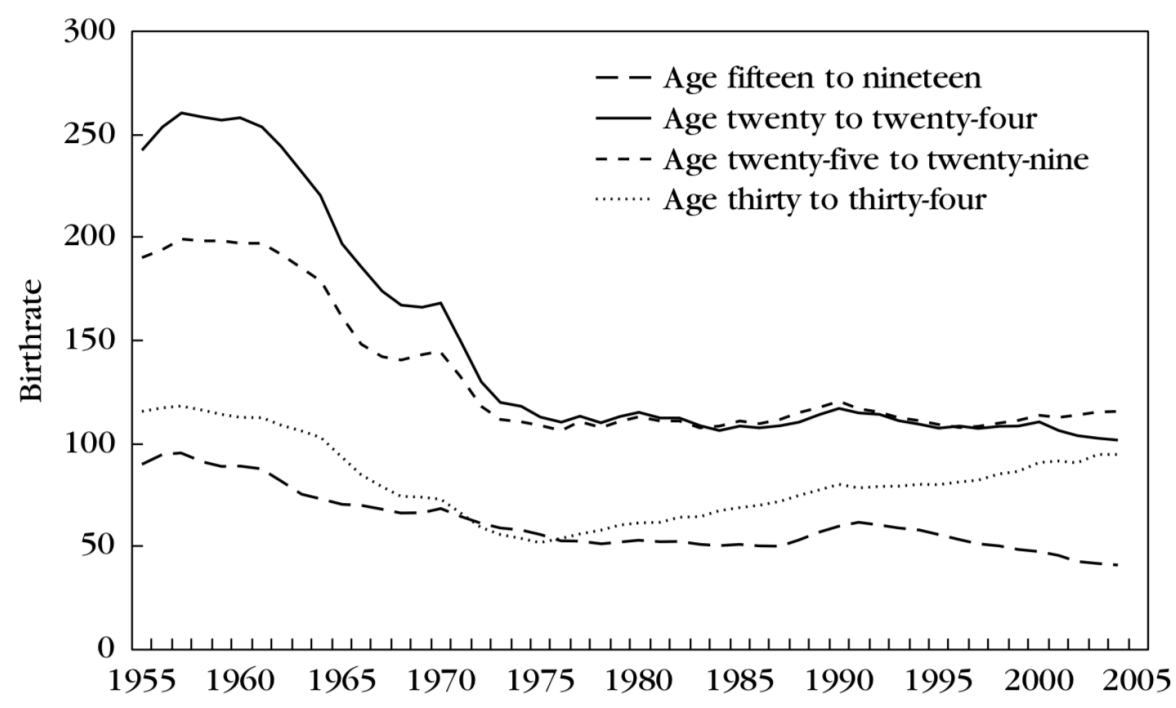

Researchers have concluded that it was not the rate of teenage pregnancy that was driving cultural panic, but the ratio of babies born to mothers out of 
wedlock. Significantly fewer teenagers were having children, but more of those who did, were (and are) electing to become single parents. It appears probable that it was the disruption of the institution of marriage itself - as women's participation in society, economy and education changed - that drove collective anxiety in the US, which was then projected on to teen mothers and particularly African American teen mothers.

Up until the 1960s, childbirth and marriage were tightly coupled. Nearly half of all American teenagers who married in the 1950s were pregnant at the time ('shotgun' weddings). Alternatives to marriage were not attractive - abortion was illegal and backyard abortion highly risky. A thriving market of white couples seeking white babies, made adoption a popular remedy for pregnant white teens. Single pregnant black women who chose to bear their baby, tended to foster their child with extended kin and community. But beginning in the mid 1960s, teen mothers began to work out en masse that early marriage, particularly to an undereducated and/or unemployed partner with low prospects was neither a necessity nor necessarily a good solution to pregnancy. In the longitudinal study of teen mothers in the 1960s, over half of the teens had married the father of their first child, but at 30 year follow-up only 1 in 5 of the marriages had survived $^{362}$. Increasingly, women of all ages and ethnicities were recognising that single parenthood was a viable option following pregnancy compared to an undesirable marriage, adoption or abortion. ${ }^{356}$

Whereas popular notions suggest that teen childbearing leads to poverty and disadvantage, it has been increasingly demonstrated that [many] teen mothers come from poor and disadvantaged families themselves, and studies of sisters [only one of whom became pregnant as a teenager] have shown that even when controlling for family of origin, teen motherhood has no significant impact on future socioeconomic advancement. ${ }^{366}$

\section{Biological Risks of Teen Pregnancy}

There is broad biomedical consensus that teen pregnancy is a risk factor for adverse health outcomes. The World Health Organisation estimates that complications during pregnancy and childbirth are the leading cause of death in females aged between 15-19 years globally (but WHO admits that this is an extrapolation from source data that does not actually provide information for this age range). As a corollary, 3.9 million females in this age range undergo unsafe abortion procedures per year ${ }^{367}$. Many large studies in both industrialised and low-to-middle income countries have reported increased risks for pre-term delivery and low birth weight in teen pregnancy. ${ }^{368}$

However other important adverse outcomes cannot be explained by lower maternal biological age - for example, a large study of over 20,000 live births to teen mothers in the urban Sahel in Africa, revealed that strictly biological factors 
- such as maternal 'biological immaturity' - were 'surprisingly unimportant' in relationship to early childhood mortality ${ }^{369}$. Another mid-sized study in Ethiopia, compared birth outcomes of more than 600 teen mothers to more than 700 older mothers and revealed that childhood survival was mostly a function of a mother's socioeconomic status rather than of her age ${ }^{370}$. A large US obstetric study reported that rates of adverse neonatal outcomes such as birth trauma, low Apgar score, infection and asphyxia in adolescent mothers were comparable to those of mothers aged 20-24, together with a positive outcome of higher rates of vaginal delivery relative to caesarian delivery in teen mothers. ${ }^{371}$

In industrialised countries, younger maternal age is associated with a constellation of risk factors including being unmarried, primiparous, undereducated, an ethnic minority, socio-economically disadvantaged, and less likely to obtain early prenatal care - all of which are associated with adverse pregnancy outcomes ${ }^{372}$. Thus mother and child birth and life-course outcomes are not simply reducible to maternal age but are functions of complex entangled dynamics between biology and environment.

\section{Weathering}

Pioneering research in the 1980s revealed that, contrary to orthodox belief and opposite to the pattern observed in white women, birth outcomes for African American women living in high-poverty areas were actually better for teenage mothers compared to women having their first child in their twenties or later. The lowest risks for low-birth weight, preterm birth and infant mortality were recorded for mid to late-teenage mothers with birth risk increasing linearly after that with maternal age. For example, in some communities, infants with 25 yearold mothers have double the risk of low-birthweight compared to infants born to 15 year-old mothers. ${ }^{364}$ Because infant health is a robust predictor of maternal health ${ }^{373}$, it appeared that the women living in high-poverty areas were significantly less healthy at age 25 than they were at age 15 .

The 'Weathering' hypothesis was proposed to explain this phenomena - that early and chronic exposure to uncontrollable, inescapable and/or toxic levels of stress such as systemic discrimination and historical intergenerational disadvantage, coupled with the costs of tenacious high-effort coping by families and communities, exacted a physiological cost that manifested as accelerated ageing and increased disease vulnerability - leading to African American teenagers struggling with conditions such as diabetes and high blood pressure that typically aren’t observed in whites until they're much older. ${ }^{374,352}$

In recent years, pathfinding research has validated the Weathering hypothesis, demonstrating significantly elevated biomarkers of allostatic load - a physiological index of exposure to adversity and of the body's consequent biological burden of stress - in African Americans relative to whites. Two 
classes of biomarkers are used to measure allostatic load - primary mediators consisting of neurochemicals released in response to stress such as norepinephrine, epinephrine, cortisol, and dehydroepiandrosterone sulphate and secondary markers produced in the wake of chronic stress including elevations in systolic and diastolic blood pressures, cholesterol levels, glycated haemoglobin levels, and waist-to-hip ratio. Allostatic load is predictive of adverse health outcomes including cardiovascular disease, functional decline, frailty, and all-cause mortality - and thus is a valuable subclinical indicator of disease risk. ${ }^{375}$

In a nationally representative US sample of over 5000 people, aged between 1864 , researchers demonstrated stark ethnic disparities in allostatic load - with blacks having significantly higher mean allostatic load scores than whites at all ages. The disparity could not be explained by poverty/ socioeconomic status with non-poor blacks having significantly worse allostatic load than poor whites. There was little difference in allostatic load scores by gender for whites until around 55 years when white women's scores rose moderately relative to white men. Black women had elevated allostatic load scores relative to black men at all ages. The differences between white and black women were pronounced. Consistent with the Weathering hypothesis, the mean score for blacks in each age group was comparable to the level for whites who were 10 years older. ${ }^{376}$

Figure: Probability of adverse allostatic load score by age, ethnicity and genderibid

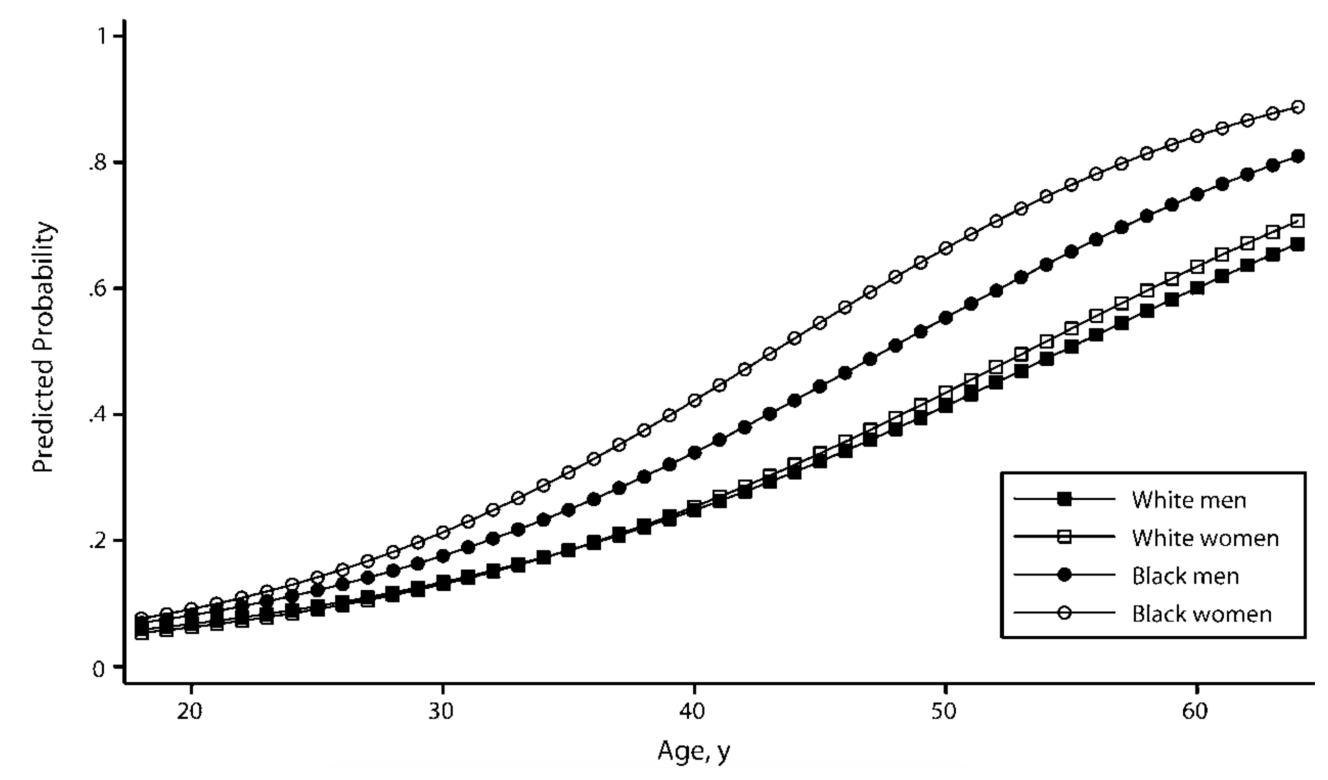

Another dimension of weathering is evident within cellular DNA. Telomeres are the protective end-caps of chromosomes, composed of repeated noncoding sequences of the amino acids TTAGGG. Telomeres are evolutionary ancient conserved in all vertebrates - and are believed to have arisen from a common ancestor about 400 million years ago. In certain cell types such as germ and 
stem cells, telomeres are primarily enzymatically maintained, but most somatic cell types lack this mechanism and telomeres consequently degrade over successive cell divisions (mitosis). The progressive shortening of telomeres constitutes a molecular clock counting down the lifespan of the cell ${ }^{377}$. Considerable evidence indicates that cellular ageing is not a function of chronological time but of 'mitotic' time - the number of divisions that a cell undergoes producing successive cell generations. ${ }^{377,378}$

DNA in the cell is subject to continuous attack from inside and outside the body. Each of the body's trillions of cells $\left(\sim 10^{13}\right)$ sustains tens of thousands of DNA lesions per day. For example, the ultraviolet frequencies of strong sunlight can induce 100,000 lesions per cell per hour. Damage, erosion and degradation of DNA occur both from normal physiological (endogenous) processes including replication errors, spontaneous chemical reactions such as oxidation, hydrolysis and methylation, metabolism of environmental toxins, reactions to inflammation and infection and from exogenous forces such as ultraviolet and microwave radiation, tobacco compounds and food contaminants. The biological consequences of DNA lesions can be severe - including disruption of critical genetic functions such as protein transcription, mutation and breakdown of cellular and systemic viability. ${ }^{379,380}$

Organisms have evolved effective and efficient cellular mechanisms collectively called the DNA damage response - to detect and repair the many different kinds of DNA lesions that arise in the course of cellular life. Shortening of a cell's telomeres beyond a critical threshold triggers a chronic DNA damage response which in turn triggers cellular death (apoptosis) or senescence (permanently arrested growth) - considered to constitute the cellular basis of ageing. Telomere induced cellular ageing is likely adaptive in mutagenic environments by limiting the dissemination of accumulated DNA errors and mutation (including tumour growth). Notably, telomere repair activity is initiated by cancer cells to escape senescence, achieve functional immortality and proliferate. ${ }^{379,381,377}$

Animal and human studies demonstrate that shorter telomeres and elevated rates of telomere degradation are associated with increased morbidity and mortality, whilst longer telomere length has been associated with a healthier physiological profile. Premature telomere shortening has been associated with a broad range of risk factors for adverse health outcomes including early life adversity such as childhood exposure to violence and abuse, smoking, obesity, psychosocial stress, chronic inflammation, mental illness, hypertension, cardiovascular disease, diabetes, cirrhosis and cancer and premature mortality. ${ }^{316,377,382,383}$

The Weathering hypothesis asserts that blacks are biologically older (prematurely aged) than whites of the same chronological age, due to disproportionate exposure to adversity and high-effort coping with stressors ${ }^{316}$. 
Telomere length at birth is equivalent in blacks, whites and Hispanics ${ }^{384,385}$. A study which examined telomere change over 4 decades compared infant telomere length (from preserved cord blood) with adult telomere length and demonstrated that blacks have significantly accelerated shortening of telomeres relative to whites between birth and adulthood ${ }^{386}$, consistent with other studies which have reported significant shortening of telomeres in black men in a doseresponse relationship to internalised and externalised experiences of ethnic discrimination and accelerated biological ageing in middle-aged black women relative to white women of the same chronological age..$^{387,316}$

\section{The Adaptive Repertoire}

Copious evidence demonstrates that across many forms of life including single celled organisms, plants, insects and animals - organisms have evolved capabilities to monitor their environment and alter their bodies, behaviours, growth, development and life-course events including the timing of reproduction and the number of offspring in order to adapt to changing conditions ${ }^{388-391}$. This adaptive capability is called phenotypic (meaning expression) variability because it occurs in the life course of the individual and is determined or 'programmed' by conditions in the environment and not mandated by the genotype. Phenotypic variability enables individuals and sub-populations within a single species with essentially the same genetic makeup to express different structural and functional adaptations (variability) to different and changing environmental conditions. Phenotypic variability is thus an evolved capability of a species that enables individuals and populations to calibrate their biological processes to local environmental conditions.

The course of development can be different from what it would be otherwise in an environment which contains, say, thalidomide, lithium ions, a psychotic parent, hot plasma, too many litter mates, or a scarcity of food. Differences in such environmental variables produce different phenotypes (or none at all) just as do differences in DNA sequences. Therefore developmental information resides in the environment as well as in the genome. ${ }^{392}$

Phenotypic variability is widespread in nature in response to predator-prey dynamics $^{393}$. For example crabs change their claw size between moults as a function of their diet - growing larger claws for predominantly hard-shelled prey such as molluscs and shrinking claw size in the presence of predominantly softbodied prey. Similarly marine snails produce thicker shells and protective ridges (apertural teeth) on the inside opening of their shells in response to the prevalence of predators such as crabs in their environment ${ }^{394}$. Different species of the water flea genus Daphnia, which can reproduce parthenogenically ${ }^{395}$ (producing genetically identical clones), demonstrate diverse kinds of adaptations to different predators - changing their bodies, behaviour and rates of development ${ }^{396}$. A well-studied example is the water flea Daphnia 
lumholtzi, which when exposed to chemical cues (kairomones) of predators in the environment, dramatically alters its body structure to protect against predation. $^{397}$

Figure: Scanning electron micrograph of genetically identical clones of the water flea Daphnia lumholtzi - the clone on the left was exposed to predator cues which induced the development of a 'horned-helmet' and elongated tail spine while the clone on the right was unexposed. . $^{\text {id }}$

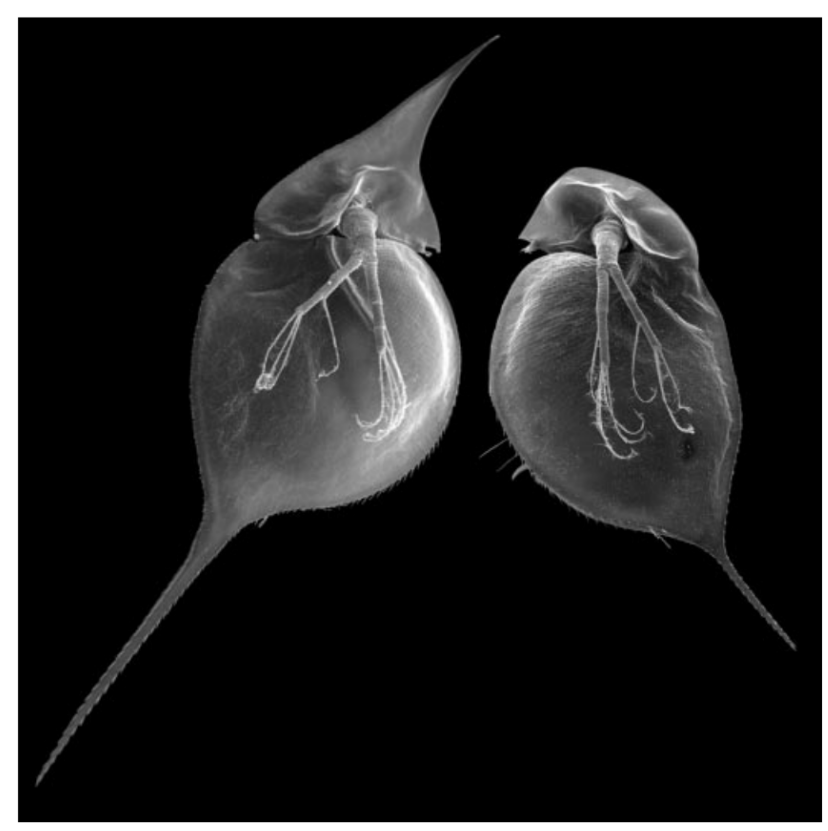

Originally observed in the wild and believed to be different species (genotypes), these individuals represent phenotypic variability within a single species and within a single generation induced by environmental differences.

It may appear prudent for natural selection to favour the defensive 'armoured' phenotype/variant to win out over the undefended phenotype and become the prevalent form in all environments but this would likely be maladaptive. Broadly speaking, species consist of diverse subpopulations distributed across different and variable environments - with each local habitat presenting different compositions of conditions, opportunities and adversities. Within its environmental range, species must contend with both risk - the predictable features of the environment that lead to good or bad outcomes and uncertainty due to the unpredictable features of the environment. While there can be persistent and prevalent risks across all the environments a species inhabits, the presence of variability and uncertainty between and within environments can make it maladaptive for a species to become fixed into a locally optimal physical form or pattern of behaviour because what may be adaptive in one local environment may be maladaptive in another ${ }^{398}$ - in the case of Daphnia, its armoured form (phenotype) has better probability of survival if predatory fish are present in the local environment while the unarmoured form has better reproductive success if they're not ${ }^{399}$. Thus to cope with variable and 
unpredictable environments, a species may select for phenotypic variability increasing its adaptive repertoire ${ }^{400}$ - its range of adaptive capabilities and responses that can be evoked by the particular conditions of the environment, rather than selecting for fixed traits. ${ }^{401}$

\section{Trade-offs}

Consider the Olympic hopeful with two performance passions: to be a great sumo wrestler and to be a great pole-vaulter. He has an obvious problem. He needs a large body mass to increase performance in sumo, but large mass is a liability for pole vaulting. Where performance in one environment is inversely related to performance in another, a functional trade-off exists. ${ }^{394}$

Life History Theory models the evolutionary and developmental strategies of diverse organisms across their life-course and describes how organisms contending with finite resources and dynamic environments must make fundamental thermodynamic tradeoffs - making resource allocation 'decisions' between different biological functions and processes in order to survive and fulfil developmental tasks. For example, 'energy' (metabolic effort) spent defending against threat in the environment such as producing an inflammatory response to fight infection cannot be simultaneously allocated to engaging with opportunities for growth, learning or recovery. Tradeoffs are made from one moment to the next as the organisms shifts functional states in relationship to changing features in its environment. ${ }^{402}$

A central tradeoff that all organisms must make according to the conditions in their environment is between investing in somatic effort: in anthropomorphic terms - the growth, development and maintenance of the body and mind and reproductive effort: performance in competition, courtship, gestation, birth and childcare. A wealth of empirical evidence across diverse taxa including singlecelled organisms, plants, insects and animals - demonstrates phenotypic plasticity in the organism's life-course including altering patterns and timetables of reproduction in response to environmental conditions ${ }^{403,404}$. For example, studies of red deer (Cervus elaphus), tawny owls (Strix aluco) and red squirrels (Tamiasciusus hudsonicus) have demonstrated changed reproductive patterns in response to adverse environmental conditions measured by variables including predator density and food scarcity. A longitudinal study of semi-captive Asian elephants (Elephas maximus) observed for over 70 years, demonstrated that females born to highly-stressed mothers had increased probability of reproduction at younger ages and accelerated reproductive senescence (agerelated decline of reproduction). ${ }^{405}$ 


\section{Teen Pregnancy as an Adaptive Response}

Contrary to the presumption that humans have low fecundity and an inefficient reproductive system, both theory and present evidence suggest that we may actually have very high fecundity and a reproductive system that has evolved to be flexible, ruthlessly efficient and, most importantly, strategic. ${ }^{406}$

Amassing evidence indicates that the relatively elevated rate of teen pregnancy observed in African American women is not due to bad (flawed or dysfunctional) biology, behaviour or culture but is an evolved adaptive response to their disproportionate exposure to systemic risk from living in an environment in which they are routinely subjected to historical, continuous and systematic discrimination and disadvantage.

Harsh and unpredictable environmental conditions constitute reliable signals of threat to future survival and future reproductive opportunities. Life History Theory describes earlier reproduction as adaptive in risky and uncertain environments ${ }^{379}$ and predicts an accelerated reproductive timetable, increased fertility and reduced parental investment in individual offspring in harsh environments. ${ }^{380}$

According to an evolutionary-developmental model, the human reproductive system has evolved to detect and respond to environmental risk and uncertainty in order to promote the survival of the species. Accordingly, the experience of early, severe and/or chronic adversity can trigger an evolved (biological) adaptive response to decrease investment in somatic effort (such as growth, health and recovery) and accelerate reproductive maturation and timing ${ }^{381}$. Evolved adaptive responses are phenotypic changes triggered by features of the environment which alter an organisms developmental trajectory - for example causing the organism to arrest or disinvest in long-term development such as somatic growth and to accelerate its timetable for life-course tasks such as reproduction.

Stress and adversity have always been part of the human experience. ${ }^{398}$

The average age that women have their first child varies across cultures - for example in the US it is at 25 years old, in Japan 29.2 years old and in Turkan in Kenya it is 22.2 years old. But differences in fertility timing are much greater within countries than between countries - for example in the US, 10\% of first births occurred in women over 35 years and $21 \%$ occurred in women under the age of 20 - likely indicating a phenotypic variability driven by epigenetic factors such as socioeconomic conditions rather than genetic factors. ${ }^{402}$

In contemporary hunter-gatherer societies - regarded as a model for human demographic patterns prior to the agrarian revolution, almost half of all children perish before reaching adulthood ${ }^{407}$. A global sample of contemporary forager, 
horticultural and agricultural populations reveals that average maternal age at first birth is between 15.5-20.5 years, compared to an average age of 25.1 in the USA and 29.9 in Canada. Contemporary teen birth rates in traditional forager and horticultural societies vary between 135-279 per 1000 women aged 15-19, compared to industrialised societies with a high of 43.0 per 1000 in the USA and a low of 5.0 per 1000 in Switzerland. It appears likely that early sexual maturity and reproduction was both adaptive and normative in ancestral populations back to our hominid forebears. ${ }^{408,409,398}$

A large longitudinal study of white women in Britain, examined the timing of first birth in relationship to neighbourhood quality - assessed by variables including income, employment, health deprivation, education and skills, barriers to housing and services, crime, and the overall environment - and demonstrated that the timing of fertility varied according to the level of deprivation in the neighbourhood so that women began childbearing significantly younger in disadvantaged neighbourhoods. ${ }^{410}$

A broad range of family-environment adversities including parental absence, stressful relationships, poor child-parent bonding, maternal harshness, lower socioeconomic position, low parental investment and diminished experience of family support are associated with accelerated menarche and earlier reproductive timing ${ }^{411,412}$. According to a Life History model, such adversities serve as significant signals of environmental threat, risk, uncertainty and instability - consequently triggering evolved adaptive responses and altering the course of biological development. For example, early childhood adversity (such as sexual abuse, domestic violence or imprisonment of a family member) is associated with higher adult mortality rates - indicating that the early experience of adversity is a robust indicator of future mortality risk. Confirming the predictions of Life History Theory - research demonstrates that such adverse childhood psychosocial environments are associated with an accelerated reproductive timetable ${ }^{413}$. Other research has demonstrated that the effects of moving home frequently (which are correlated with other risk factors such as family instability and loss of support networks) has far reaching consequences for children including adverse health outcomes, higher adult mortality rates, increased substance abuse, symptoms of mental illness and poor educational performance. A mid-sized study of adolescents in Britain, demonstrated that the frequency of moving home had a cumulative biological effect - girls who had experienced 1-4 or 5 or more residential relocations had double and triple the likelihood the probability of reaching menarche at a given age compared to girls who had not relocated home. ${ }^{412}$

\section{Broken Models, Broken Policies}

In overview, beginning around the late 1960's, reaching a crescendo in the 1990's and continuing into the present, teenage pregnancy became widely 
denounced in the US, UK and internationally as a significant social problem and public health threat ${ }^{414}$. The scientific evidence-base indicates that teenage pregnancy is strongly associated with socioeconomic inequality and unstable and deprived family environments. Contrary to the dominant narrative that presented teenage pregnancy as a primary cause of poverty, the preponderance of evidence shows that poverty precedes pregnancy - as deprivation, risk and inequality are commonly significant factors in a young woman's life years before teenage pregnancy occurs. ${ }^{415,416}$

A large literature has developed documenting poor health and life-course outcomes for mother and child following teenage pregnancy. But this literature is criticised for not adequately controlling for critical factors such as socioeconomic status and maternal smoking - and when these are taken into account the evidence indicates that mid to late-teen mothers (16-18yrs) may actually require less obstetric intervention, have less risks of conditions such as intrauterine growth retardation, pre-eclampsia and eclampsia and are no more likely to have still-born or underweight infants than otherwise similar women who have their first child aged between $18-34$ years $^{415}$. Furthermore, broad evidence indicates that maternal and child life-course outcomes including educational attainment and employment are not adversely scripted by teenage pregnancy. ${ }^{359,361,363,414}$

Public policy efforts to reduce rates of teen pregnancy have been largely unsuccessful ${ }^{413,417}$, with many intervention programs aimed at reducing the rate of teen pregnancy continuing to be disseminated, despite having been repeatedly shown to be ineffective ${ }^{418-420}$. A dominant discourse models teen pregnancy as an outcome of moral, behavioural and/or cognitive failure that can be corrected through education, training and indoctrinating normative cultural values - for example through teaching sexual abstinence, contraceptive use or setting lifegoals. ${ }^{417}$

Americans have imagined that the persistence of poverty and other social problems can be traced to youngsters who are too impulsive or too ignorant to postpone sexual activity, to use contraception, to seek an abortion, or failing all that, especially if they are white, to give their babies up for adoption to "better" parents. Defining the problem this way, many Americans, including those in a position to influence public policy, have come to believe that one attractive avenue to reducing poverty and other social ills is to reduce teen birth rates. Their remedy is to persuade teenagers to postpone childbearing, either by convincing them of the virtues of chastity (a strategy conservatives prefer) or by making abortion, sex education, and contraception more freely available (the strategy liberals prefer). ${ }^{360}$

Teen pregnancy is typically modelled as a kind of failure on the part of individuals, groups and even entire cultures - essentially a predominant 
narrative characterises teen pregnancy as the outcome of (some combination of) bad characters, from bad backgrounds, doing bad things and making bad choices. Accordingly, research, policy and intervention programs are predominantly oriented to identifying and correcting problematised or ('deficient') individuals and groups through educating or indoctrinating normative thinking, behaviour and values. These efforts have been resoundingly unsuccessful. The overall failure of public policy and programs to reduce teen pregnancy in some of the wealthiest nations in the world, provides strong evidence of the failure of underlying models and the compelling need to reexamine their guiding assumptions.

Broad cross-species evidence demonstrates that accelerated reproductive development is a biologically driven process that is adaptive to adverse environmental conditions. Consequently, it appears that teen pregnancy disparities within and between countries are not driven by culture or conscious choice but by primarily unconscious biological responses to exposure to systemic risks - and accordingly attempting to 'remedy' high rates of teen pregnancy by 'rectifying' teen behaviour, values, attitudes, behaviours, practices and thinking is unlikely to change population-level patterns of reproduction. A Life History model indicates that effective interventions for high rates of teen pregnancy require addressing the systemic risks that compose the environment driving evolved adaptive responses across population groups. ${ }^{413}$

\section{Complexity and Mental Health}




\section{Part Four: Evolution}

\section{Evolved Mechanisms underlying Common Psychological Conditions}

Figure: Theoretical foundations of the FEAR model

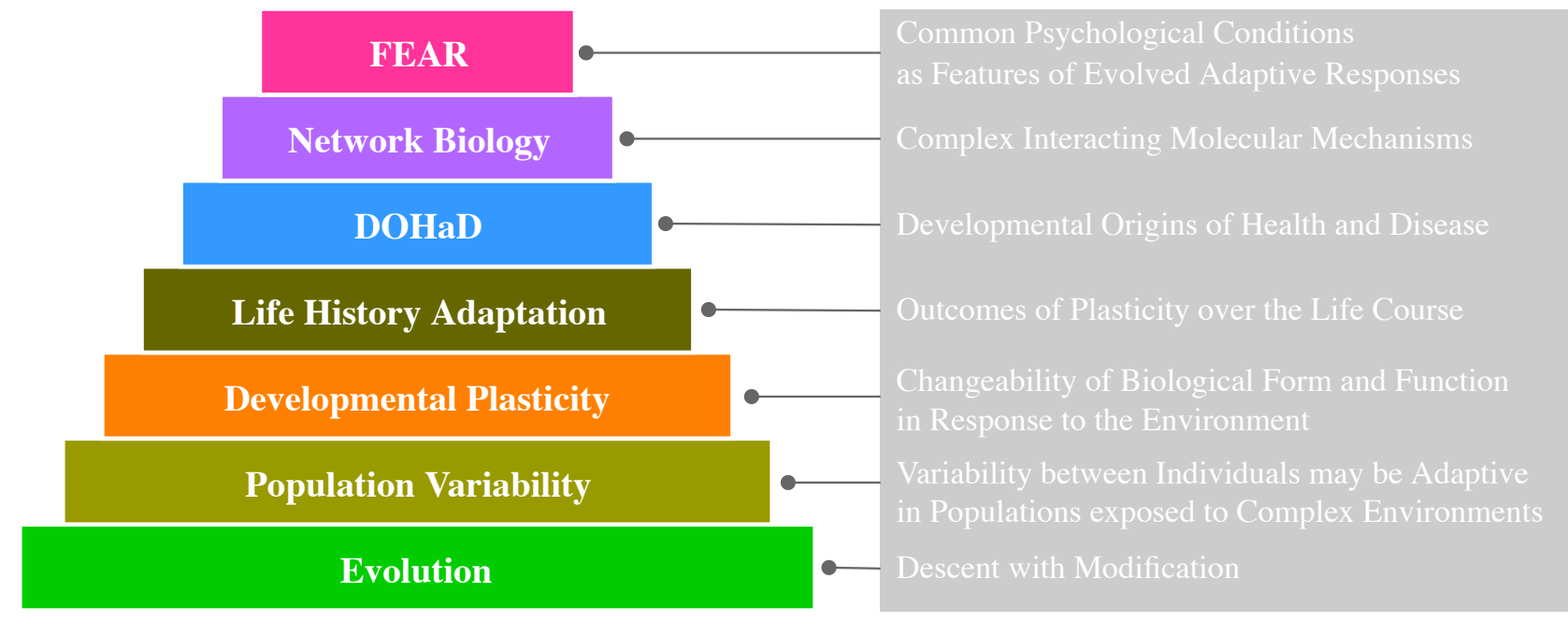

Preponderant evidence across taxa demonstrates that common environmental exposures and normal experiences mark biological systems; persistently and profoundly

- shaping patterns of genetic expression

- changing biological form and function including morphology, physiology, cognition, emotion and behaviour

- determining patterns of health and disease

- in sum: altering the individual phenotype. Biological marking by exposure and experience - how the environment gets under the skin - although pronounced in early development, occurs throughout the life-course - and is rapid, organised and widespread via molecular signalling pathways which connect perturbations and features of the environment with a broad array of cellular mechanisms which modulate genetic expression - for example by switching genes on and off.

The shaping of genetic expression and phenotype by exposures and experiences throughout the life-course produces a natural spectrum of individual developmental strategies, pathways and trajectories within populations. Developmental plasticity and inter-individual variability can be adaptive in populations because in the volatile, complex and dynamic conditions which characterise the environments of many organisms, there can be no single, stable and universal - 'one-best-way' - path of development. What is locally adaptive in one set of conditions may be maladaptive when conditions change and vice versa. Instead, preserving a range of phenotypes may support population fitness. Thus phenotypes and life-course trajectories which 
may be locally sub-optimal for the individual in terms of wellbeing and longevity may be spatially and temporally adaptive for populations exposed to complex variable environments and hence conserved in the species.

The FEAR model proposes that common psychological conditions are outcomes not of pathological processes but of evolved mechanisms including natural population variability and phenotypic switching produced by developmental plasticity. Furthermore, the effects of such evolved adaptive responses (at individual and population levels) can be transmitted across generations so that the aetiology of common psychological conditions - so-called 'mental disorders' - can originate at levels superordinate to the individual such as the family, community and population.

Unravelling the evolved mechanisms which connect the environment and patterns of biological response and expression paves the way for a new model of mental health and presents new pathways for intervention and recovery from common psychological conditions.

\section{Evolution}

The morphological, physiological, and behavioral characters of modern humans arose through hundreds of millions of years of vertebrate evolution. Most of the basic mechanisms that govern human development and physiology are present in primitive extant vertebrates, and even invertebrate species...Thus, it is not surprising that the adaptive solutions that humans have developed are derived evolutionarily from mechanisms that were in place in the earliest vertebrates. ${ }^{421}$

An emerging evolutionary-developmental model proposes that common categories of mental disorder including psychological trauma are not forms of biological failure meaning breakdown or pathology (disease, disorder, dysfunction or dysregulation), but are forms of biological function - the psychological features of evolved adaptive mechanisms that have been conserved in evolution by providing survival advantages to the species despite adversely impacting individual wellbeing.

nothing makes sense in biology except in the light of evolution ${ }^{422}$

Theodosius Dobzhansky

Evolution has been broadly defined as

any net directional change or any cumulative change in the characteristics of organisms or populations over many generations - in other words, descent with modification $^{423}$

In the $21^{\text {st }}$ century, the scientific understanding of the nature and mechanisms of evolution is undergoing tectonic upheaval, echoing the collapse of the orderly Newtonian model of the universe as its bedrock of stability, predictability and certainty gave way to a much more turbulent cosmos characterised, post-Einstein, by complexity, instability and unpredictability. 
Figure: Evolving Scientific Models of Evolution ${ }^{424-426}$

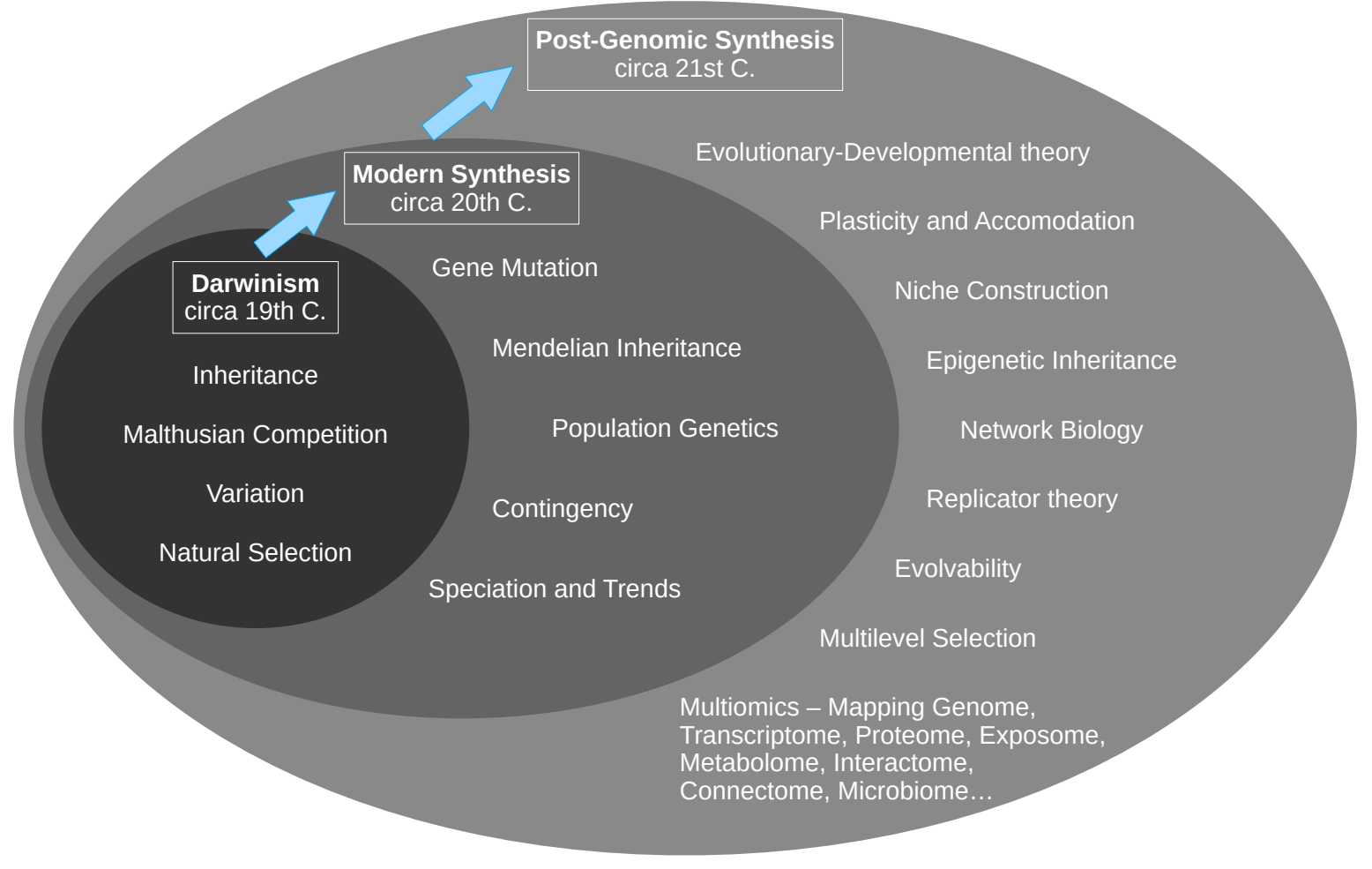

In the post-genomic era, all the major tenets of the modern synthesis have been, if not outright overturned, replaced by a new and incomparably more complex vision of the key aspects of evolution. ${ }^{427}$

The basic building blocks and concepts of evolutionary biology - the gene and its functions, the species, the organism, the role of the environment and adaptation - are being radically remodelled and rewritten in the post-genomic era. ${ }^{428-431}$ For example, the human body is composed of about 100,000 different kinds of protein, which are thus crucial for life and are continuously reproduced by the processes of gene expression (transcription of DNA into RNA and translation of RNA into protein). ${ }^{432,433}$

A living cell is a continuing, action-packed drama. Molecules interact and change. One change triggers another, and so on and on. Complex chains of molecular interaction happen again and again. We call them 'pathways'... And proteins form the backbone of all these biochemical pathways. Cells organise into tissues, such as skin, bone, muscle, to form organs such as the heart and kidneys, and finally, all these, together with the immune and hormonal systems, form the organism, the whole animal. This operates in many different ways, at various levels of organisation. And all of this 'function', as biologists say, involves proteins. ${ }^{433}$

In 2004, the International Human Genome Sequencing Consortium, led in the United States by the National Human Genome Research Institute and the US 
Department of Energy, published its findings after finishing its monumental task of sequencing the human genome $\mathrm{e}^{434,431}$ - the entire set of protein-coding genetic instructions found in chromosomal and mitochondrial ${ }^{435}$ DNA $^{\phi}$. Following the tenets of the Modern Synthesis, researchers had predicted that the human genome would contain around 100,000 genes - composing the minimum necessary instruction set for generating observed human complexity - our traits, commonalities, differences and critically - our predispositions and patterns of health and disease ${ }^{434,436}$.

For more than 50 years scientists have operated under a set of seemingly incontrovertible assumptions about genes, gene expression, and the consequences thereof. Their mantra: One gene yields one protein; genes beget messenger RNA, which in turn begets protein; and most critically, the gene is deterministic in gene expression and can therefore predict disease propensities. ${ }^{429}$

Instead, the project discovered that the human genome contains only about 20,000 protein-coding genes, about the same number as in the microscopic nematode worm Caenorhabditis elegans, only about twice as many as in a fly and less than the genetic complement of a sea sponge (which lacks a gut and nervous system) or a cow, 5 times less than an onion and 200 times less than the single-celled Amoeba dubia ${ }^{431,437-439}$. Subsequent research has demonstrated that the complexity of an organism (in terms of parameters such as number of differentiated cell types, levels of functional organisation, development or behaviour) does not map simply to its genetic endowment. ${ }^{440-442}$

Genetic determinism - a legacy of the dogma of the Modern Synthesis - which asserts that genes 'code for' or provide the 'blueprint' for biological form and function (phenotype) has been replaced in the post-genomic era by a far more complex map between the gene and biological expression - demonstrating that living forms and functions are not products of genetic dictation, but of a continuous conversation between the genome, the body and the world - so that what is biologically expressed is not what is written in the genes but what is read from the genes through regulation, interactions and exposures. ${ }^{443-445}$

some very influential twentieth-century biologists presented a simplistic genecentred view of biology using memorable metaphors and brilliant writing to encourage you to adopt their view. And in this they were very successful. Hardly any biological discovery today is presented in the popular media without reference to the discovery of this or that gene 'for' something or other... [but in reality] there are no genes 'for' anything. Living organisms have functions which use genes to make the molecules they need. Genes are used. They are not active causes. ${ }^{446}$

$\phi \quad$ The human mitochondrial genome was first sequenced in 1981. 
Pre-Genomic Era

Fixed Genome (genes in stable positions on the chromosome like beads on a string)

1 gene $\rightarrow 1$ protein

A species is defined by a unique genetic pool ${ }^{450}$

Tree of Life (branching speciation) $)^{453}$

Only vertical gene transfer within species

Genomes are sleek (finely honed) genetic

libraries (evolution acts like an engineer) ${ }^{456}$

Genetic inheritance (the Weisman barrier)

Species are finely adapted to their environments, thus there is little overlap between their genomes and common features across different species such as the photoreceptors of eyes evolved independently ${ }^{440}$

Non-protein-coding ‘junk’ DNA

Genetic Determinism - genes code for expression (phenotypes)

Focus on the presence of genes

Read-only genome (Central Dogma) $\rightarrow$ popularised as genes are the central processor or brain of the cell

Evolution is driven by random small genetic mutations honed by Natural Selection ${ }^{463}$

\section{Post-Genomic Era}

Fluid Genome - mobile DNA sequences (transposons) can move around the genome, have been identified in all organisms and make up $\sim 45 \%$ of human genome $e^{447,448}$

1 gene $\rightarrow$ can produce many proteins with diverse functions ${ }^{449}$

The majority of life forms on Earth - the prokaryotes and viruses - do not conform to the concept of species because they form huge genetic information transfer networks pangenomes ${ }^{451,452}$

Web of Life (differentiation and interconnection $)^{429}$

Horizontal gene transfer across genomes ${ }^{454,455}$

Genomes are messy and produced through bricolage (evolution acts like a tinkerer) ${ }^{434}$

Genetic and Epigenetic inheritance ${ }^{457,458}$

Ancestral 'toolkit' genes are shared across diverse forms of life such as clustered Hox genes which provide information for the basic body plans of all multicellular animals and Pax transcription factors involved in eye development $\mathrm{t}^{459,460}$

Non-protein-coding DNA is diverse and can be regulatory, structural, a reservoir for evolvability or junk $k^{461,440,441}$

Genetic Regulatory Networks ${ }^{433}$ - phenotypes are determined by biological networks

Focus on the expression of genes ${ }^{462}$

Read-Write genome $\rightarrow$ organisms control or regulate their genomes ${ }^{458}$ and thus the genome is not the cell's brain but its factory producing components of cellular machinery as directed by the cell and the organism ${ }^{446}$

Non-random adaptive jumps are prevalent ${ }^{463}$ through mechanisms such as targeted mutation $^{458,464}$ and large evolutionary jumps occur through mechanisms including transposons ${ }^{458}$, horizontal transfers across species and symbiogenesis 
Figure: from genetic dictation to network conversation ${ }^{465}$

Modern Synthesis

Phenotype

(expression)
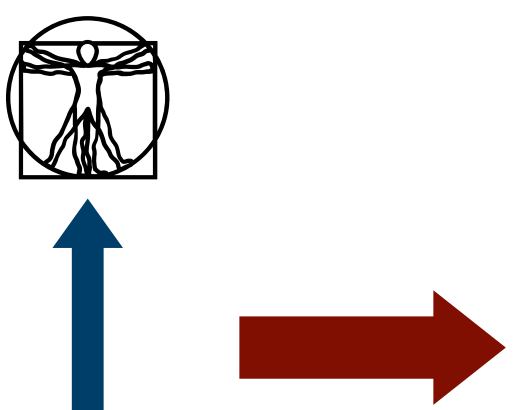

Post-Genomic Synthesis

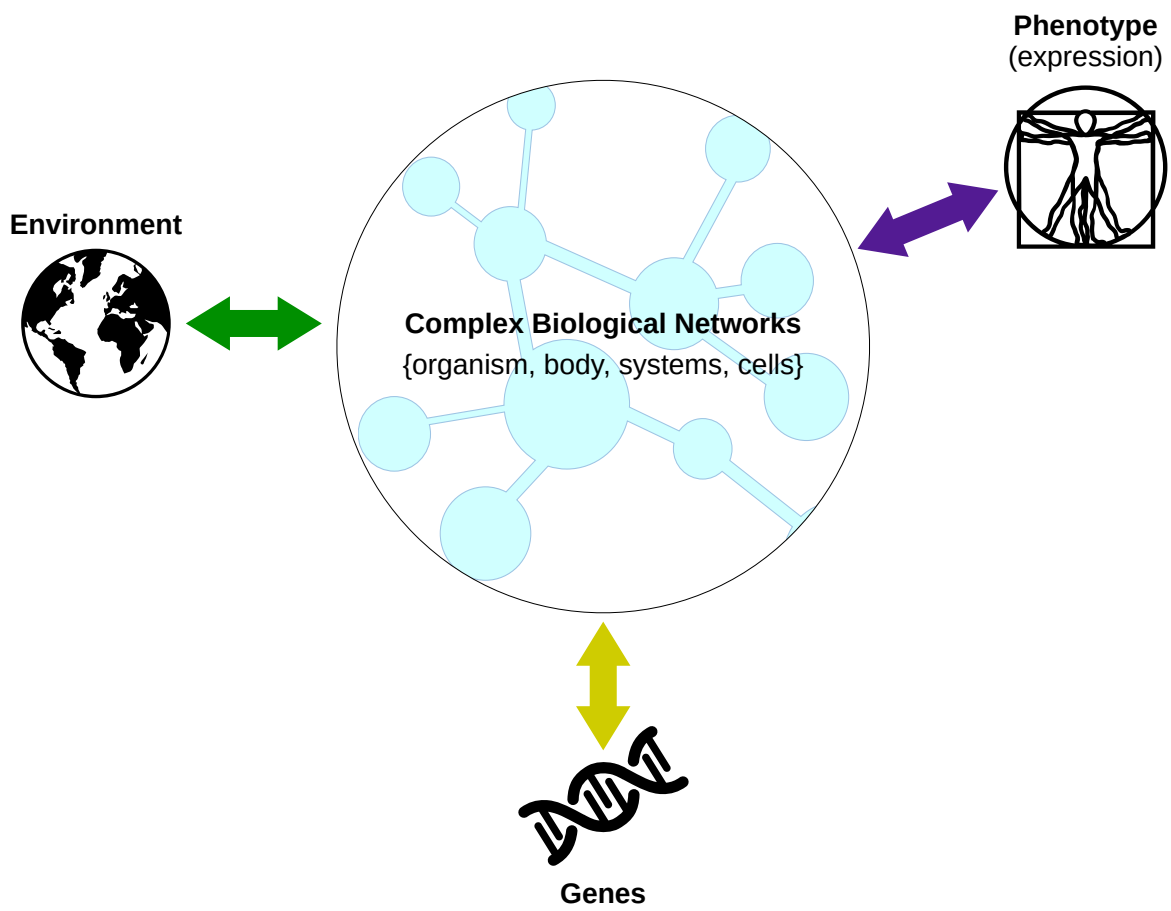

In essence, the present 'omics' era - of the mapping of the genome, transcriptome, proteome, interactome, microbiome, exposome, connectome, epigenome and so forth - has displaced the gene from centre-stage of the biological universe and driven a transition from a gene-centric to a networkcentric modelling of biological function, development and evolution which can be broadly described as Network Biology. ${ }^{446,466}$

Figure: A small biological network - an interactome map of protein-protein and protein-DNA (red-dot) interactions in the 'minimal' bacteria Treponema pallidum ${ }^{\Gamma}$ showing the molecular interactions of a subset of 576 of its 1039 different proteins. ${ }^{467}$

$\Gamma \quad$ T. pallidum has one of the smallest known genomes of any extracellular bacteria 


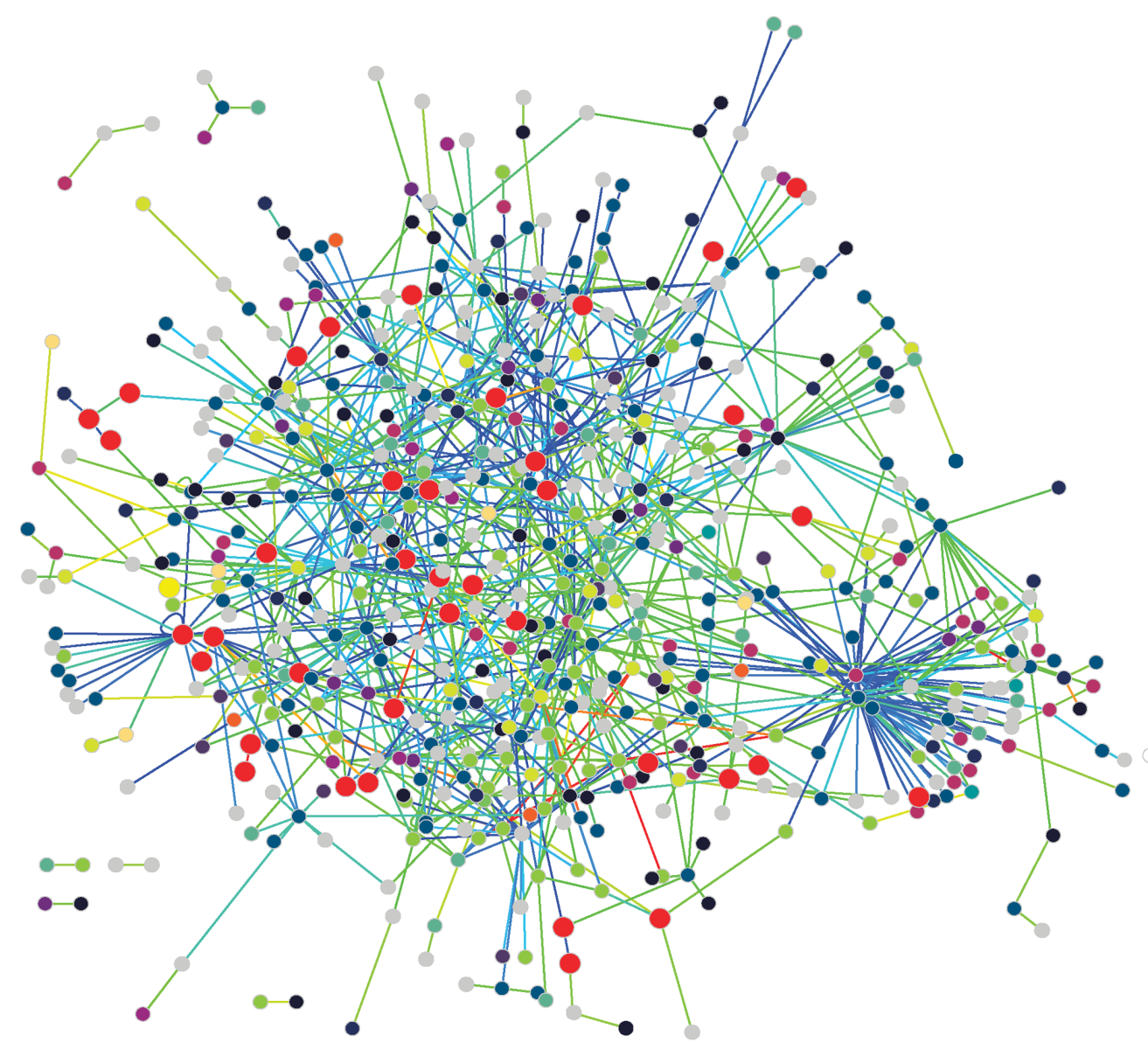

\section{Developmental Plasticity}

In biology, plasticity is defined as

the ability or an organism to react to an internal or external environmental input with a change in form, state, movement, or rate of activity. ${ }^{392}$

In other words, plasticity is the ability to change biological form and function in response to environmental conditions - and appears to be a universal property of living systems. ${ }^{392}$

Phenotype is considered to be all the traits of an organism other than its genome - in other words, what is expressed in terms of biological form and function. Development may thus be broadly defined as all phenotypic change that occurs in the lifetime of an individual or population. And developmental plasticity may be defined as phenotypic variability, flexibility or change in the life-course of the organism in its environment. ${ }^{392}$

For example, substantial evidence demonstrates that wild fish populations (including Halibut, Pacific salmon, North Sea plaice, Northeast Arctic cod, Baltic cod and Atlantic cod) alter their growth rates $^{\Psi}$ and reproductive timing in

$\Psi \quad$ Fish growth rate is highly plastic and complex - driven by many factors such as temperature and predator/prey distributions. 468 
response to large-scale commercial fishing. The higher mortality rates of mature fish (due to selective human predation) create a strong environmental pressure which induces adaptive change in the fish population towards earlier age and decreased size at first reproduction. Similar effects have been observed in populations of ungulates (such as deer) which have been exposed to sporthunting, and in fruitflies under experimental manipulation. ${ }^{409,468}$

Figure: Adaptive developmental plasticity of fish reproduction in response to commercial explotiation $^{413}$

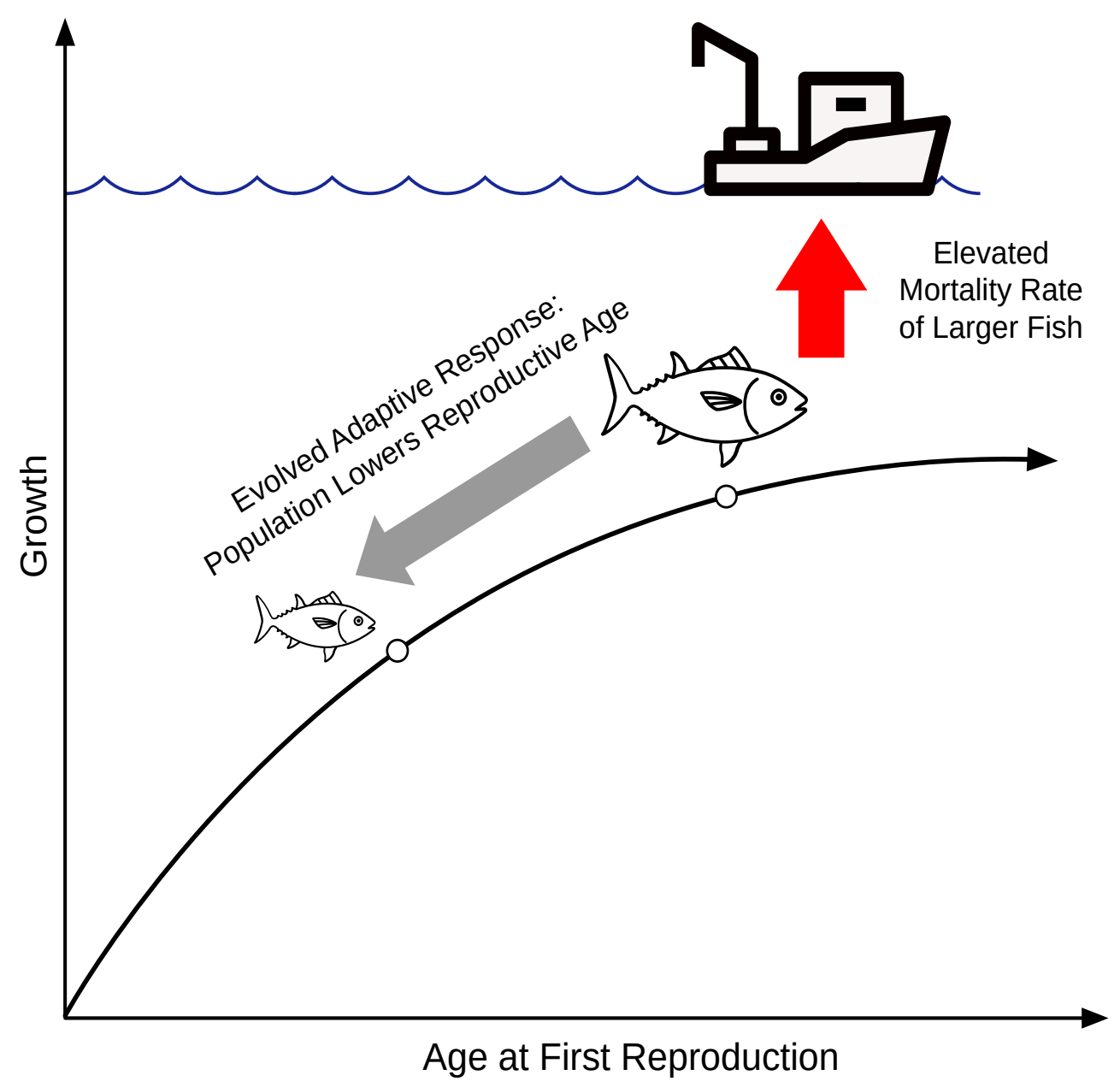

Developmental or phenotypic plasticity can be broadly described as the ability of living organisms to - sometimes dramatically - alter their form (morphology), functions (physiology) and behaviour in response to the environment, producing persistent changes in their life-course trajectory and life-history outcomes such as patterns of health and disease ${ }^{379}$. Developmental plasticity is observed across all biological taxa and its effects can span generations ${ }^{497,502,503}$. 
Figure: Environmentally triggered phenotypic plasticity (polyphenism) across diverse $\operatorname{taxa}^{504}$
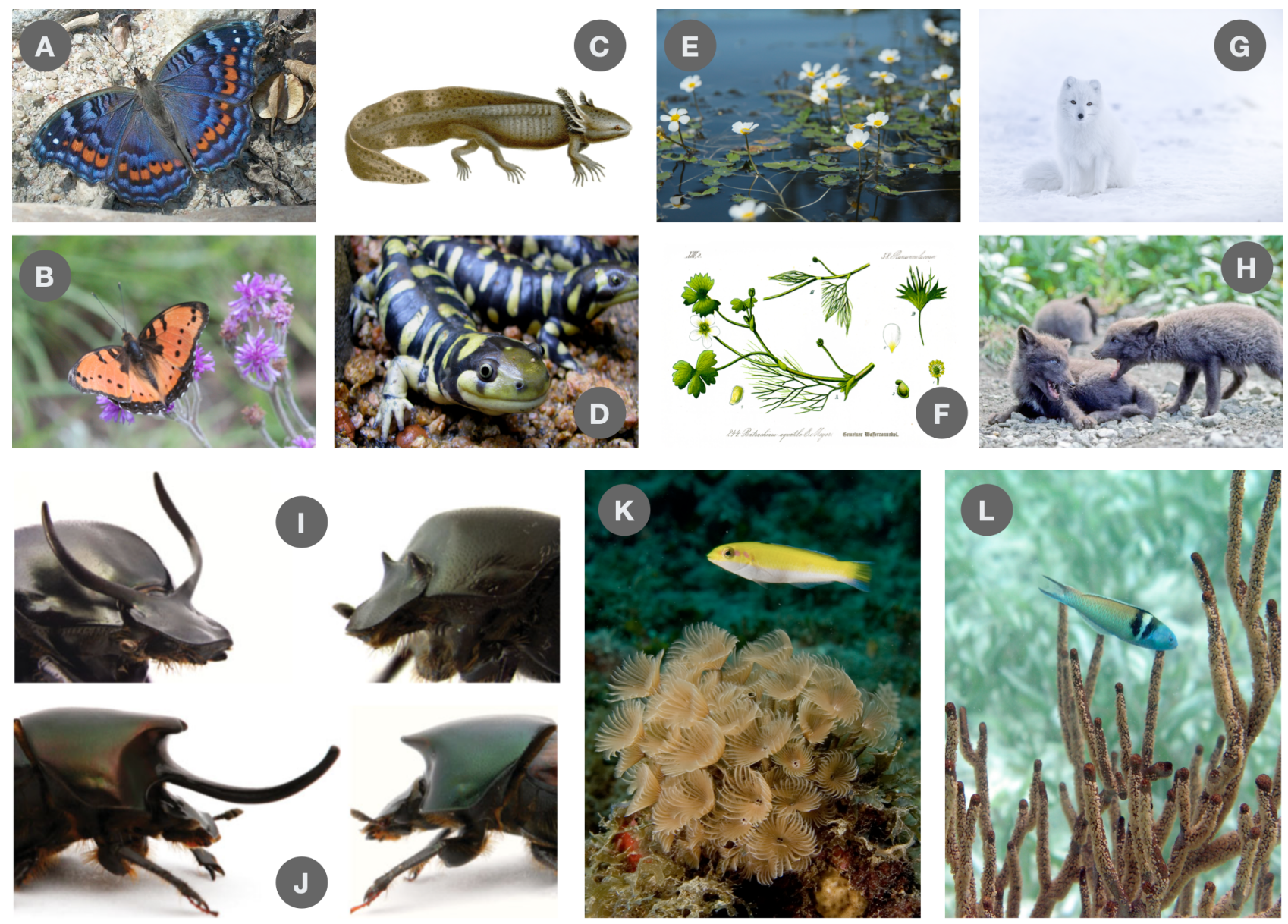

The Gaudy Commodore (Precis octavis) is a native African butterfly that is seasonally dimorphic - individuals which emerge in the dry season are blue-winged (A) and individuals which emerge in the wet-season are orange-winged (B). Tiger Salamanders (genus Ambystoma) are native North American amphibians which demonstrate facultative (discretionary) metamorphosis - adults remain in an aquatic larval form $(C)$ and only transform into a terrestrial form (D) if the aquatic environment becomes uninhabitable. The White Water-Buttercup (Ranunculus aquatilis) is native throughout Europe, northwest Africa and northwest America and forms different shaped leaves above and below water. Surface leaves are fanning and scallop-shaped (E), while submerged leaves form needlelike branching structures (F). The Arctic Fox (Vulpes lagopus) is a small fox native to Arctic regions of the Northern hemisphere including northern Europe, northern Asia and northern America. A white-furred variant $(G)$ is the most common form (morph) comprising $99 \%$ of the inland population, while a blue/brown/grey-furred variant $(H)$ is more common in coastal regions. Male Dung Beetles (subfamily Scarabaeinae, genus Onthophagus) develop into large horned forms or smaller hornless forms which have different morphological, physiological and behavioural characteristics - depending on parental provisioning of an ample or scarce food store to the developing larvae $(I, J)$. The Bluehead Wrasse, a small saltwater fish native to the coral reefs of the western Atlantic ocean, demonstrates protogynous hermaphroditism in which a smaller/immature female form $(K)$ can become a larger male form $(L)$ in response to population pressures. ${ }^{469-482}$ 
Increasingly, developmental plasticity is understood not as a function determined by genes but as a function of the interactions between the organism and the environment:

The genome is therefore today best described as a "vast reactive system" embedded in a complex regulatory network with distributed specificity. An important part of this regulatory network is involved in responding to environmental signals, which can cover a very broad range of phenomena, from the cellular environment around the DNA, to the entire organism and, in the case of human beings, their social and cultural dynamics... Genes are now seen as "catalysts" more than "codes" in development, "followers" rather than "leaders" in evolution. The more genetic research has gone forward, the more genomes are seen to "respond in a flexible manner to signals from a massive regulatory architecture that is, increasingly, the real focus of research in 'genetics'”, 483

\section{Metamorphosis}

Classically defined as a spectacular and typically abrupt transformation of a larval form into an adult form, such as a caterpillar becoming a butterfly - metamorphosis provides a remarkable example of developmental plasticity. Many animals, including amphibians and most teleost fish (bony fish with prehensile jaws and symmetrical tails - which make up $96 \%$ of all extant fish and about half of all vertebrate species) undergo metamorphic change - radically altering their form (morphology), function (physiology), behaviour and habitat. ${ }^{484,485}$

The majority of amphibian species have an aquatic larval stage (such as a tadpole) which is followed by metamorphosis into a terrestrial adult form (such as a frog). Most amphibian larva are hatched into temporary water bodies such as rain pools, crevices or even bamboo stumps - where a chief risk to larval survival is desiccation and drying out of the habitat. Amphibians have evolved to exploit such highly unpredictable and uncertain aquatic habitats by decoupling somatic growth from maturation - enabling them to demonstrate extreme developmental plasticity. In favourable conditions with abundant food sources, low predator risk and minimal habitat desiccation, amphibian larvae grow in size but decelerate or arrest maturation (meaning developmental progression ${ }^{486}$ ). In adverse conditions, such as rapid habitat desiccation and drying, larvae arrest growth and accelerate maturation - bringing forward the timing of metamorphosis in order to escape the adverse conditions. ${ }^{487}$

Figure: Developmental plasticity by decoupling growth and maturation 


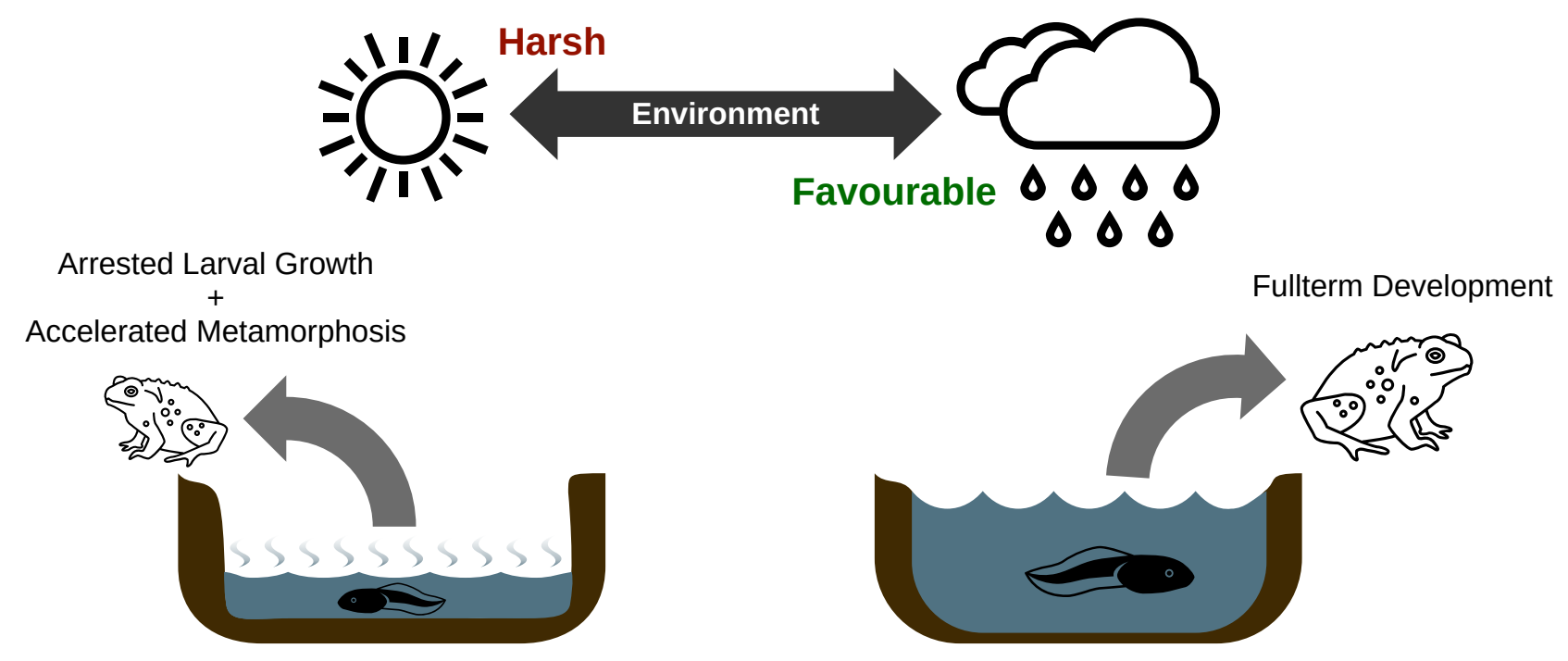

However, the gambit of developmental acceleration incurs significant physiological, morphological and future developmental (so-called 'life-history') costs - because tadpoles that metamorphose early to escape a desiccating pond consume a large portion of the stored fat that is essential for future hibernation in their adult form, fail to accumulate additional fat reserves and consequently transform into frogs with smaller limb size, altered immune response, delayed and smaller size at first reproduction and reduced probability of survival. ${ }^{487,488}$

\section{The Pathology of Preterm Birth}

More than one in 10 of the world's babies born in 2010 were born preterm (defined as birth before 37 weeks of gestation) - resulting in about 15 million premature births globally, with rates increasing in nearly all countries with reliable data, despite increasing medical knowledge of risk factors associated with preterm birth and the introduction of many public health and medical interventions in wealthy industrialised nations such as the USA. More than one million babies died as a direct consequence of their prematurity while many of the survivors face significant lifelong disabilities ${ }^{489}$. The risks of preterm birth include perinatal mortality, long-term neurological disability, admission to neonatal intensive care, severe morbidity in the first weeks of life, prolonged hospital stay after birth, readmission to hospital in the first year of life and increased risk of chronic lung disease. Additionally, preterm birth can exact a severe psychological toll on families. ${ }^{490,491}$

Preterm birth represents the most significant problem in maternal-child health in the United States. It is the leading cause of infant mortality and morbidity; its prevalence in our population is unacceptably high and has not decreased over the last 40 years; and its etiology is unknown in a substantial proportion of cases. ${ }^{492}$

Some of the highest rates of preterm birth are observed in the United States with around $11 \%$ of white infants and $18 \%$ of black infants born prematurely ${ }^{493}$. Similar social disparities are observed in Australia, where approximately $14 \%$ of babies of 
Indigenous mothers are born preterm, compared with $8 \%$ of babies of non-Indigenous mothers. ${ }^{491}$

Very little is known about the causes and mechanisms of preterm birth, and without this knowledge, preterm birth will continue. Before pregnancy, some solutions are known to prevent preterm birth such as family planning, especially for girls in regions with high rates of adolescent pregnancy; yet there are few other effective prevention strategies available for clinicians, policy-makers and program managers. Once a woman is pregnant, most of the interventions to prevent preterm birth only delay onset, turning an early preterm birth into a late preterm birth.

\section{World Health Organisation, $2012^{490}$}

$70 \%$ of preterm births are idiopathic (meaning of unknown cause) with the remaining $30 \%$ due to maternal or fetal infection or maternal hypertensive conditions (typically leading to iatrogenic preterm birth - meaning medically induced delivery or caesarian section $)^{494,495}$. A constellation of social and behavioural factors are known to be associated with increasing risk of preterm birth $^{\delta}$ - including teen pregnancy, low socioeconomic status, diet and nutrition, lack of prenatal care, domestic violence and smoking ${ }^{496}$, but these risk factors have been shown to play only a limited role in driving preterm birth ${ }^{492}$. The mechanisms which translate social risks into biological outcomes remain unidentified.

Considerable empirical evidence demonstrates that the experience of high levels of psychosocial stress during pregnancy significantly increases the risk for preterm birth by between $25-60 \%$, even after controlling for established social, biomedical, and behavioural risk factors ${ }^{492}$. Social determinants play a critical role in the aetiology of preterm birth. For example, a 'healthy migrant effect' is widely reported in the US, where recent African and Hispanic migrant women have lower risks of preterm and low birthweight babies compared with African-American and Hispanic-American women ${ }^{497}$. Adverse perinatal outcomes significantly increase in Mexican women after settlement in the USA - women who have been resident for more than 5 years have a 1.9-fold greater risk of delivering preterm, and a 1.5 greater risk of a low birthweight infant compared to newly arrived Mexican women ${ }^{498}$. Moreover, the health degradation effect follows a clear ethnic gradient that mirrors the ethnic pattern of socioeconomic inequality in the US - with the strongest effects observed amongst African migrants, followed by Hispanic migrants and virtually absent among Asian and European migrants. ${ }^{497}$

Further evidence of the primary role of the social determinants of birth outcomes is emerging from the experiences of Indigenous women in colonised nations:

Aboriginal or indigenous women, especially those in the United States, are overwhelmingly classified as high-risk...Birth has become dangerously

Other adverse birth outcomes include fetal growth restriction (low birth weight) and post-term birth 
medicalized for them. Cut off from traditional diets, support networks, and community midwives due to colonization and assimilation, many Native women have chronic health conditions that mean giving birth is a high-risk activity—and one that requires travel to well-equipped hospitals...The practice of forcing Native women to travel to hospitals because their traditional ways of caring for pregnant people were outlawed contributes to an endless cycle of poor outcomes. $^{499}$

Indigenous women in colonised countries including Canada, Australia, the USA and Taiwan have significantly worse pregnancy outcomes including low birthweight infants, preterm birth, neonatal and perinatal mortality and stillbirths, compared with non-aboriginal women ${ }^{500}$. A global social movement over the last four decades has organised to return birthing to Indigenous communities. For example, in Canada -

the community driven initiative to establish the Inuulitsivik Midwifery Service in a remote Inuit community meant women were no longer flown $8 \mathrm{~h}$ away for birth. This change was seen as an important contributor to the cultural healing required to address the impact of colonisation which had weakened the health, strength and spirit of the community. Evaluation data reported high community engagement, local training of Inuit midwives, improved birth outcomes and very low intervention rates with caesarean section facilities not available on site. Subsequently there has been a return of birthing services and onsite midwifery training to other remote Inuit communities as they gain control of their health services and address the social determinants of health with holistic services and employment and education initiatives for First Nations women ${ }^{501}$

No Indigenous birthing centres exist in Australia despite national policy recommending their establishment. A 5-year prospective cohort study of an Indigenous birthing service in Brisbane, Australia compared birth outcomes of both Indigenous women and non-Indigenous women with an Indigenous partner who received either standard Western antenatal healthcare or who received an Indigenous birthing service called 'Birthing in Our Community' - designed, led and implemented with Indigenous partnership, including Indigenous staff such as midwives and family support workers. After controlling for confounding risk factors such as smoking, maternal health conditions such as hypertension, renal, liver or heart disease and previous adverse pregnancy outcomes such as preterm birth, caesarian section or stillbirth, the birth outcomes of 345 women receiving standard care were matched with those of 345 women receiving the Birthing in Our Community service. 5 year data revealed that the preterm birth rate approximately halved in the women receiving the Indigenous birthing service relative to those receiving standard Western biomedical care (7.5\% vs $13.9 \%)$ - effectively eliminating the gap in preterm and term birth outcomes between Indigenous and non-Indigenous women. ${ }^{501}$ 


\section{Idiopathic Preterm Birth as Evolved Adaptive Response}

Remarkable surveillance and response systems have evolved and are conserved in many species, ranging from desert-dwelling Western Spadefoot toads to humans, so that they can detect threats to survival during early development and adjust their developmental trajectories. When tadpoles detect the evaporation of life-sustaining pools of desert water, their metamorphosis accelerates to ensure their survival. The human fetal-placental complex has evolved similar mechanisms to sample information from maternal circulation: If the prenatal environment is perceived to be stressful or hostile, the fetal-placental complex may promote accelerated developmental trajectories, such as preterm birth, that ensure short-term survival. ${ }^{502}$

Although pathological mechanisms remain unidentified in $70 \%$ of preterm births, emerging empirical evidence demonstrates that addressing the 'upstream causes' or social determinants of health can significantly reduce or perhaps even resolve the massive preterm birth societal inequalities observed in countries including Australia and the US.

Amassed evidence indicates that a primary mechanism of idiopathic preterm birth is not a pathological process but an evolved, adaptive and plastic response to a volatile, uncertain and complex environment. Research in evolutionary-developmental biology points to developmental plasticity as a central mechanism underlying variability in human birth timing and enabling the powerful effect of social determinants to 'shift the needle' of birth outcomes.

Fetal development is a logarithmic process, with rapid mitosis at early stages and cellular hypertrophy and accumulation of fat, glycogen, and connective tissue later in gestation. It is well-established that there are several sensitive or critical periods in development, and there may be critical periods during pregnancy of altered vulnerability to the effects of prenatal stress...The state of pregnancy is [also] associated with major alterations in maternal biology, including changes in stress-sensitive endocrine, immune and vascular processes and control mechanisms (feedback loops). ${ }^{492}$

Both mother and fetus play active roles in the timing and process of birth (parturition) $)^{492,493,503}$. Evidence from evolutionary-developmental and molecular biology reveals crucial mechanisms governing parturition. According to the evolutionary record, amphibians were the first vertebrates to adapt to life on land. The amphibian tadpole and the human fetus both undergo a similar developmental transition from an aqueous to a terrestrial environment, which requires the development of lungs for respiration and limbs for locomotion. A common neuroendocrine axis (a system combining neural and hormonal signalling) ${ }^{504}$, conserved in vertebrate evolution for at least 300 million years, governs this transition - and regulates both amphibian metamorphosis and human birth. ${ }^{505,506}$ In tadpoles, thyroid hormone initiates the profound biochemical and morphological changes of metamorphosis which is also accelerated by glucocorticoids (a form of 
corticosteroid or so-called 'stress hormone') that are produced by the interrenal

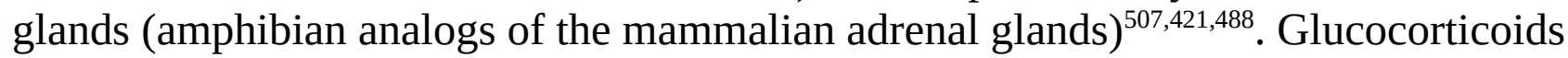
have multidimensional (profound and powerful) actions and effects in vertebrates regulating metabolism, cellular fluid balance (osmoregulation), physiological and behavioural adaptation, core end-organ function and gene transcription ${ }^{508}$. The amphibian hypothalamus produces corticotrophine-releasing hormone (CRH) ${ }^{\diamond}$ which regulates both the production of thyroid hormone and of corticosteroids and thus forms the central mechanism enabling the tadpole to regulate its development and the timing of metamorphosis in response to the environment. ${ }^{488}$

$\mathrm{CRH}$ is the central endocrine regulator of the vertebrate stress response $\mathrm{e}^{508}$. $\mathrm{CRH}$ and glucocorticoids are critical determinants of birth timing in mammals. In humans, evidence indicates that a neuroendocrine signal originating in the fetus, up-regulates (increases and drives) hormone production in the placenta and precipitates parturition. The fetal hypothalamic-pituitary-adrenal axis (a neuroendocrine system) becomes functionally mature in the $3^{\text {rd }}$ trimester - and initiates a positive feedback cycle between glucocorticoids in the fetal circulation, CRH produced by the placenta and CRH and glucocorticoids in the maternal circulation - driving an exponential increase of stress hormones in both mother and fetus, which in turn promotes the maturation of fetal organs including brain, lungs and gut and prepares the uterus and fetal membranes for birthing. ${ }^{488}$

There is substantial evidence that the placenta detects a broad gamut of maternal physiological and psychological stress signals and responds by adjusting fetal development including the timing of birth and the rate of fetal growth ${ }^{492}$. Studies of human placental cells cultured in vitro have revealed that they are highly sensitive to stress signals - releasing CRH in a dose-response relationship to all known biochemical stress effectors including cortisol, catecholamines, oxytocin, angiotensin-II and interleukin-1. A mid-sized study of 232 pregnant women in the US, aged between 18-40yrs, demonstrated that elevated CRH levels measured at 33 weeks gestation, more than tripled the relative risks of preterm birth and of fetal growth restriction (low birth weight) ${ }^{509}$.

Evolutionary evidence indicates that the hypothalamic-pituitary-adrenal/ interrenal axis (HPA axis in mammals and HPI axis in amphibians) functions as an autonomic (involuntary, automatic and unconscious) surveillance and signalling system of ancient origin. In humans, this neuroendocrine axis forms a central mechanism which transduces (converts and translates) environmental signals including conditions such as racism and poverty into biological outcomes by shaping and determining physiological, behavioural and developmental responses to the environment ${ }^{510,508,511}$.

Thus the balance of evidence indicates that a central mechanism of idiopathic preterm birth is not a pathological process but rather an evolved and adaptive response which is triggered by environmental exposures and life-course experiences.

$\diamond \quad \mathrm{CRH}$ is also commonly referred to as corticotrophine-releasing factor (CRF) 
Figure: A common neuroendocrine mechanism governing developmental plasticity in the process of human birth and amphibian metamorphosis

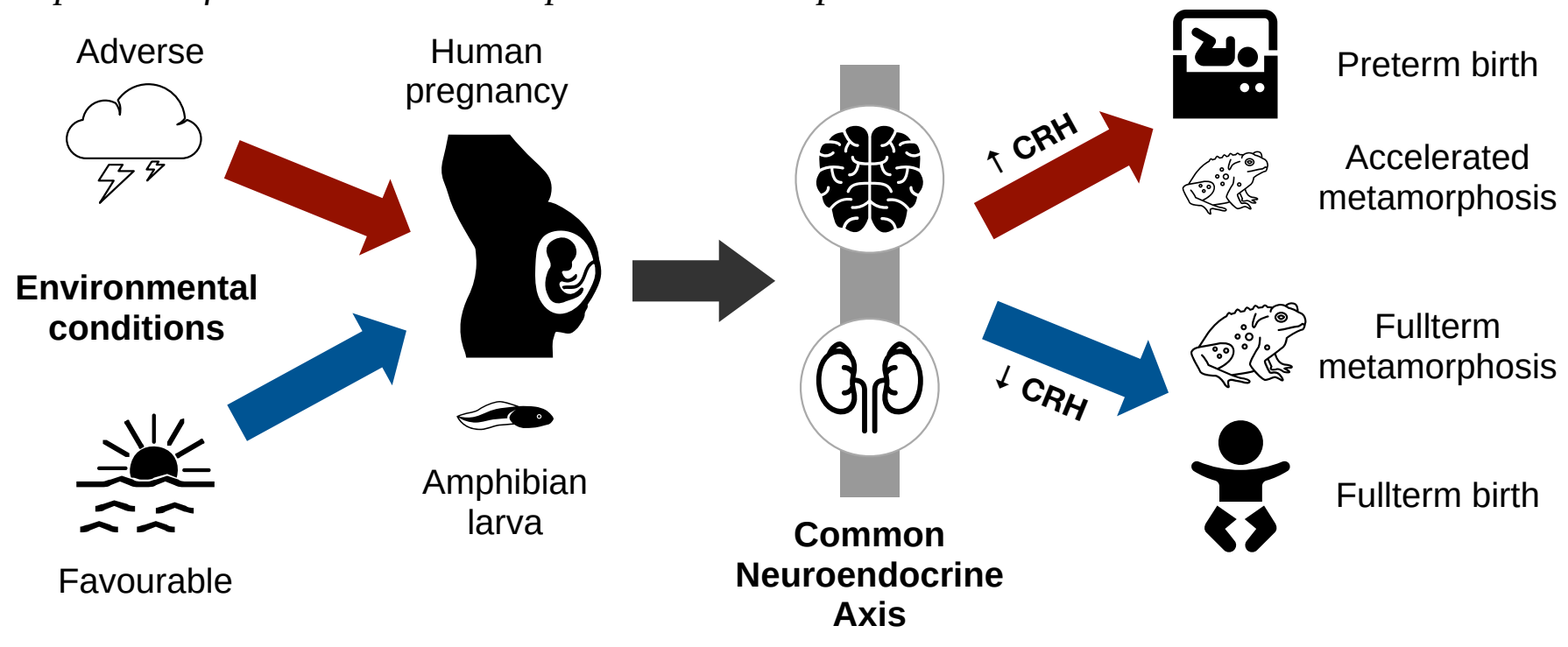

\section{Non-Communicable Diseases}

Rapid changes in global life expectancy occurred in the $20^{\text {th }}$ century - from 53 years in 1950 to 76 years in 2017 for females and from 48 years to 71 years for males, accompanied by huge reductions in early childhood mortality. Yet despite significant indications of human progress, the social world remains volatile - for example deaths from warfare and terrorism - a proxy indicator of geopolitical instability - more than doubled between 2007-2017. Alongside global demographic transition, humankind is undergoing profound epidemiological transition (meaning changing global population patterns of disease) - during the period 1990-2017 there was a 41\% decrease in communicable diseases and neonatal disorders and a $40 \%$ increase in non-communicable diseases (NCDs). The global population burden of disability increased by 52\% between 19902017 and $80 \%$ of this increase is attributed to non-communicable diseases. ${ }^{512}$

$\mathrm{NCDs}^{\Pi}$ (a catch-all term for chronic and non-contagious forms of illness) ${ }^{515,516}$ are the leading causes of death and disability worldwide ${ }^{514}$. The global burden of NCDs is increasing dramatically ${ }^{517}$. In 2016, NCDs accounted for over 70\% of global deaths. $80 \%$ of NCD deaths are due to cardiovascular diseases, cancers, chronic lung diseases and diabetes. NCDs have been long mischaracterised as diseases of affluence and age but NCDs affect people of all ages - accounting for at least $25 \%$ of all deaths in every age group above 10 years, and for more than half of deaths in age groups above 40 years ${ }^{518}$. Moreover, the global burden of disability (measured by years lived with disability) is most concentrated not in elderly but in working age people ${ }^{512}$. $80 \%$ of NCD-related deaths occur in low and middle income countries (LMICs), with the greatest burden in Asia

$\Pi \quad$ The term NCD is problematic, because it includes some diseases with infectious causes such as $~ 15 \%$ of all cancers, and it typically excludes mental illnesses, despite their large contribution to long-term disability. ${ }^{513,514}$. 
including India and China, and with rising prevalence in sub-Saharan Africa and Central and South America ${ }^{519}$. By 2030, the global economic costs of NCDs are predicted to be comparable to those of climate change and approaching those of a global fiscal meltdown - equivalent to $48 \%$ of the world's Gross Domestic Product (GDP) and driving millions into poverty. ${ }^{520}$

The currently dominant scientific and public health model for NCDs assumes that they are a consequence of genetic predisposition, coupled with adult lifestyle choices that are largely under voluntary control. However, attempts to reduce the burden of NCDs by promoting weight loss [obesity is a significant risk factor for NCDs] and changing adult lifestyles have been relatively unsuccessful at a public health level. ${ }^{519}$

The broad failure of $4^{\text {th }}$ wave public health policy and interventions targeting individual risk factors to reduce the population burden of NCDs, coupled with the broad failure to identify genetic causes for the major chronic NCDs (the so-called 'missing heritability' problem) $)^{521}$ is compelling research and policy attention towards the social and developmental origins of NCDs. ${ }^{513,519}$

\section{The Developmental Origins of Health and Disease}

Extensive experimental evidence spanning more than 3 decades of animal research and human epidemiological studies demonstrates that the environmental conditions of early development, including social and parental conditions such as attachment and bonding, profoundly shapes health and disease outcomes in later life. This field of research is known as the Developmental Origins of Health and Disease and emerged in the $21^{\text {st }}$ century as a consequence of the failure of dominant $4^{\text {th }}$ wave public health policy to stem the escalating global burden of the NCDs. ${ }^{519}$

Abundant historical, cross-cultural and empirical evidence have consistently demonstrated that low socioeconomic status is predictive of adverse birth outcomes such as preterm birth and low birthweight ${ }^{522}$. The researchers who pioneered the Developmental Origins of Health and Disease field observed that people who had been born in areas of high perinatal mortality and who had experienced adversities such as poverty, poor diet, overcrowding, and infection - demonstrated deficits in development and early growth and had increased risk of death from coronary heart disease as adults. Furthermore, these adverse outcomes persisted even in those who subsequently moved to more affluent areas ${ }^{519}$. Landmark research in the 1980's demonstrated that low birthweight and weight at $1 \mathrm{yr}$ are predictive of adult diseases including hypertension, coronary heart disease, stroke and metabolic syndrome. Together, these findings countered the widely-held belief that these major NCD conditions are primarily 'diseases of affluence'. ${ }^{519}$

Large international studies have demonstrated significant associations between birth size and subsequent adult disease profiles linked to the metabolic syndrome including 
ischemic heart disease, stroke, insulin resistance and type-II diabetes. Further research has also demonstrated associations with reduced adult bone density, schizophrenia, atopic conditions and asthma. However, birthweight is a crude indicator of prenatal development and does not by itself explain NCD risk - for example many populations with high rates of NCDs have relatively low rates of low birthweight infants. Elevation of adult disease risk has been clearly demonstrated across the range of birth sizes/weights and not just at the extremes of low and high birthweight - for example elevated risk for adult heart disease has been demonstrated in normal-range birthweight infants and structural and functional changes have been demonstrated in normal weight range fetuses as a consequence of factors such as maternal diet and body composition - including altered hepatic, celiac and splenic blood flows and fat deposition. Consequently, current research attention is shifting towards a broader investigation of development including the role of early environmental exposures - the 'exposome' - and early life experiences in the aetiology of NCDs. ${ }^{519}$

\section{The Epigenome}

...it no longer makes sense to debate nature versus nurture. There is no longer a mechanistic dichotomy between nature and nurture (or genes and environmental experience, as is the more modern phrasing). Rather, it is now clear that there is a dynamic interplay between genes and experience, a clearly delineated and biochemically driven mechanistic interface between nature and nurture. That mechanistic interface is epigenetics. ${ }^{523}$

Genetic variation between individuals - meaning structural differences between their DNA sequences - account for only a small fraction of human differences and human disease ${ }^{524,521}$. For example, large population studies have demonstrated that genetic factors explain only about $5 \%$ of human height ${ }^{521}$ - a complex trait with an estimated $80 \%$ heritability ${ }^{\#}$. Similarly, general intelligence is also considered to be highly heritable - up to $80 \%$ or more when measured in adults. Yet, very large population studies have revealed no genes that exert large deterministic effects on general intelligence. Instead, large genomic studies have demonstrated that the genetic contribution to general intelligence is the product of a large number of genes (thus intelligence is described as a polygenic trait) each exerting very small effects ${ }^{528,529}$. Despite high estimated heritability, the total genetic contribution to general

\# Heritability is a population statistic defined as the ratio of genetic variability for a given trait in a population to the range of phenotypic variability for that trait. Arguably, the quest to quantify heritability reflects the separation of nature (genotype) and nurture (environment) that is a legacy of the $20^{\text {th }}$ century Modern Evolutionary Synthesis. In the $21^{\text {st }}$ century, the low explanatory power of genetic determinants for most humans characteristics and conditions has compelled a new quest to investigate the epigenetic causes of inheritance such as the social determinants of health and disease..$^{520-522}$ 
intelligence is only between $5-7 \%{ }^{530,531}$. In the case of significant diseases such as the NCDs, the identified genetic associations contribute to only small elevations of risk $^{521,526}$. Consequently, the quest to understand significant individual differences (phenotypic variability) and population patterns of health and disease is turning from the investigation of the presence or absence of gene variants (alleles) towards investigating the activity of genes and the determinants of genetic expression. ${ }^{526}$ Organisms are biologically marked by their range of normal and abnormal exposures and experiences in their environments. Features of the environment such as the quality of parental care, the social environment, advantage and adversity can produce persistent and profound biological and developmental effects - some of which are reliably transmitted to offspring. Inheritance of traits (phenotypes) outside of genetic transmission is called epigenetic inheritance - defined as heritable changes in gene expression and in phenotype occurring without changes in the underlying DNA sequence $^{532}$.

Epigenetic mechanisms play a fundamental role in somatic development. Mammals, including humans, are complex multicellular organisms whose bodies are comprised of more than 200 different cell types that make up diverse tissues such as bone, blood, muscle, viscera and brain. Yet these diverse cell types are genetically identical - thus something more than genes determines cellular differentiation. In the early embryo, 'totipotent' cells differentiate into the diverse and specialised cell lineages that compose the body - a process regulated by the epigenome - a layer of mechanisms outside the genome which changes patterns of genetic expression and cellular development without changing the genetic sequence. ${ }^{533,534}$

Within the cells of complex organisms including plants and animals, DNA is packed into a membrane-bound nucleus where it is bound together with proteins such as histones into a complex structure called chromatin. Genetic expression in which DNA is decoded into functional proteins in order to enact the drama of life - requires the unwinding of DNA from its protein packaging to make genetic sequences available for molecular interactions and production. Conversely, the rewinding of DNA into chromatin effectively 'silences' or deactivates gene expression. Epigenetic processes include mechanisms which act on DNA and/or chromatin proteins to determine where and when genetic sequences are activated or deactivated - thus regulating genetic expression. ${ }^{535}$

Figure: DNA and proteins packaged into chromatin in the nucleus of eukaryotic cells ${ }^{536}$ 


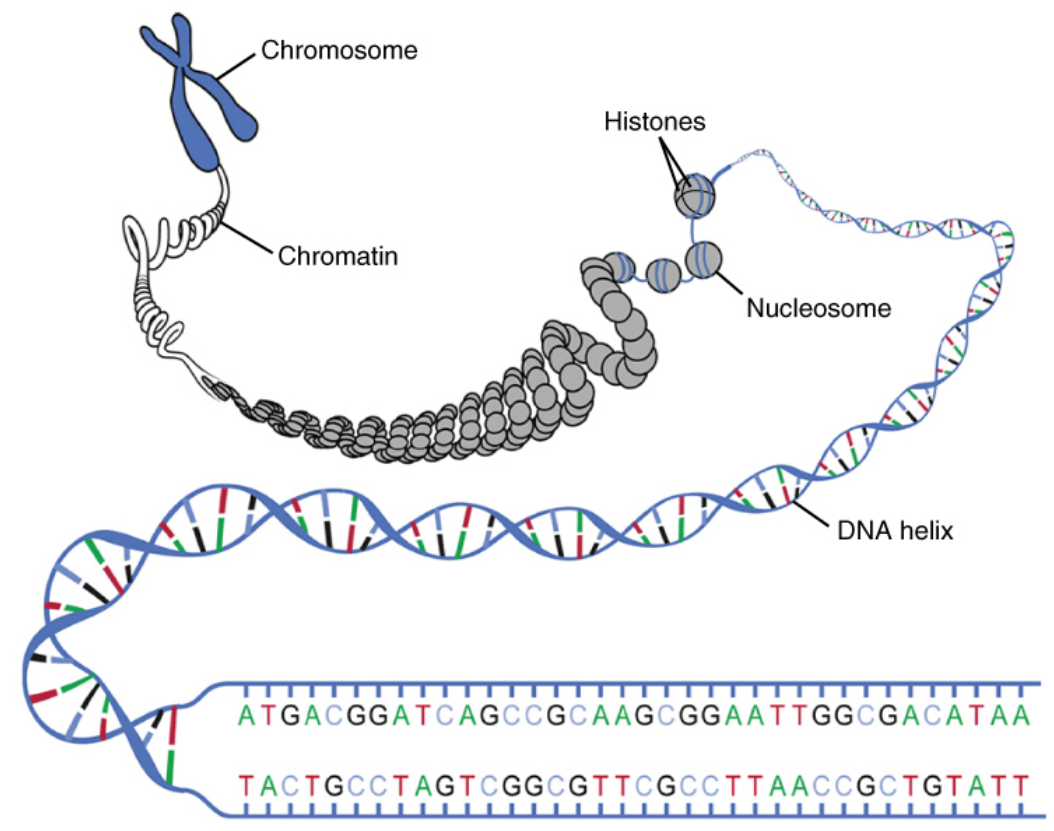

Many different kinds of epigenetic mechanisms have been described such as DNA methylation (meaning adding or subtracting methyl $\left(\mathrm{CH}_{3}\right)$ groups from DNA), modification of histone proteins through multiple processes such as methylation, acetylation, phosphorylation, ubiquitylation, and sumolyation, modification of chromatin structure and a plethora of regulatory micro-RNA molecules ${ }^{535}$. These diverse epigenetic mechanisms can act in concert ${ }^{537}$ to produce rapid, organised, widespread and persistent changes in genetic expression in response to the environment - changing biological functions and structure and driving health and life-course outcomes.

Figure: Epigenetic mechanisms shape genetic expression and drive biological outcomes ${ }^{538}$

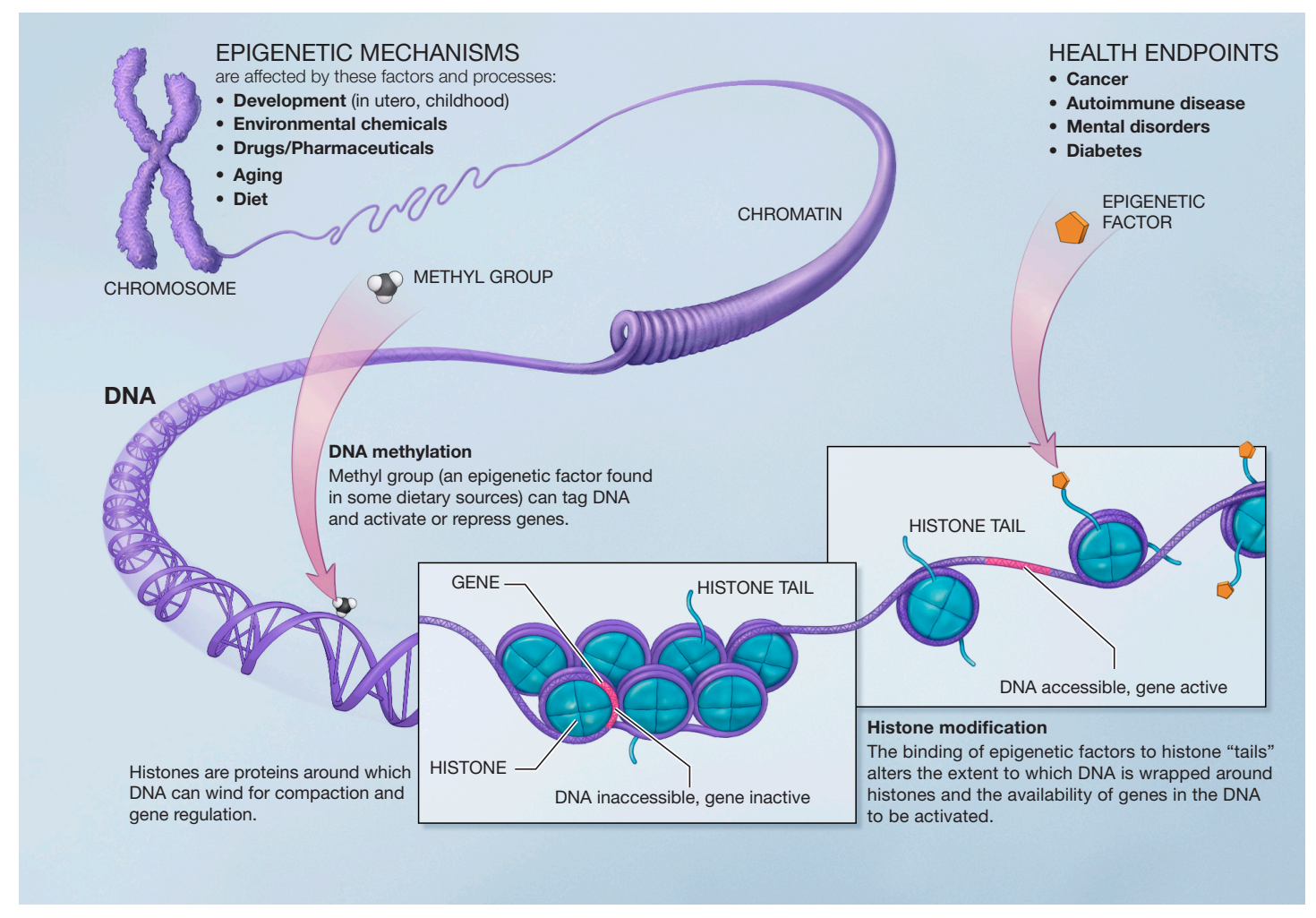


Critically, epigenetic changes have been demonstrated in the aetiology of a broad range of human pathologies - including most forms of cancer, infertility, cardiovascular, respiratory, metabolic, immunologic, and neurodegenerative diseases $^{535,539}$. Epigenetic changes often precede pathological changes and can constitute valuable diagnostic and prognostic indicators. Epigenetic drugs (epidrugs) which disrupt or reverse adverse epigenetic changes are already in clinical application and have demonstrated benefit in the treatment of some forms of cancer such as blood malignancies ${ }^{537,540,541}$. Epigenetics and the epigenome are therefore of considerable significance in investigating the aetiology of NCDs and common forms of mental disorders.

\section{Biological Marking by Exposures and Experiences}

Biologists over the ages have asked the question: does the genetic material of the fertilized egg already contain a full set of building specifications for the organism? Over the last decade or so, there has been a major paradigm shift in developmental biology regarding fundamental concepts of how the central nervous system and the rest of the organism develops and functions. The answer to the above question is now believed to be an unequivocal NO. Genes and environment are no longer considered to exert separate influences [the perennial debate about 'nature vs nurture'], and development is viewed not as a gradual elaboration of an architectural plan preconfigured in the genes, but rather as a dynamic interdependency of genes and environment characterized by a continuous process of interactions in a place and time-specific dependent manner, and involving short and long-term information storage, whereby genetic and epigenetic processes, at every step of development, become represented in the evolving structural and functional design of the organism. ${ }^{542}$

According to the tenets of Network Biology ${ }^{466}$, an individual organism's trajectory of development is neither dictated by its genes nor by its environment - but rather is an outcome of an ongoing conversation - including exposures, interactions and regulatory processes - linking the organism as a complex system with the environment, including the social, physical and chemical dimensions. Collectively, epigenetic processes represent a powerful and tangible mechanism enabling experiences and exposures to get under the skin - and become written into biological structure and function - by shaping genetic expression and driving health, disease and developmental outcomes.

It appears that epigenetic modifications such as DNA methylation that occur early in life can be system-wide and involve multiple genetic pathways ${ }^{524}$.

Figure: Epigenetic Regulation of the Genome and Phenotypic Plasticity 


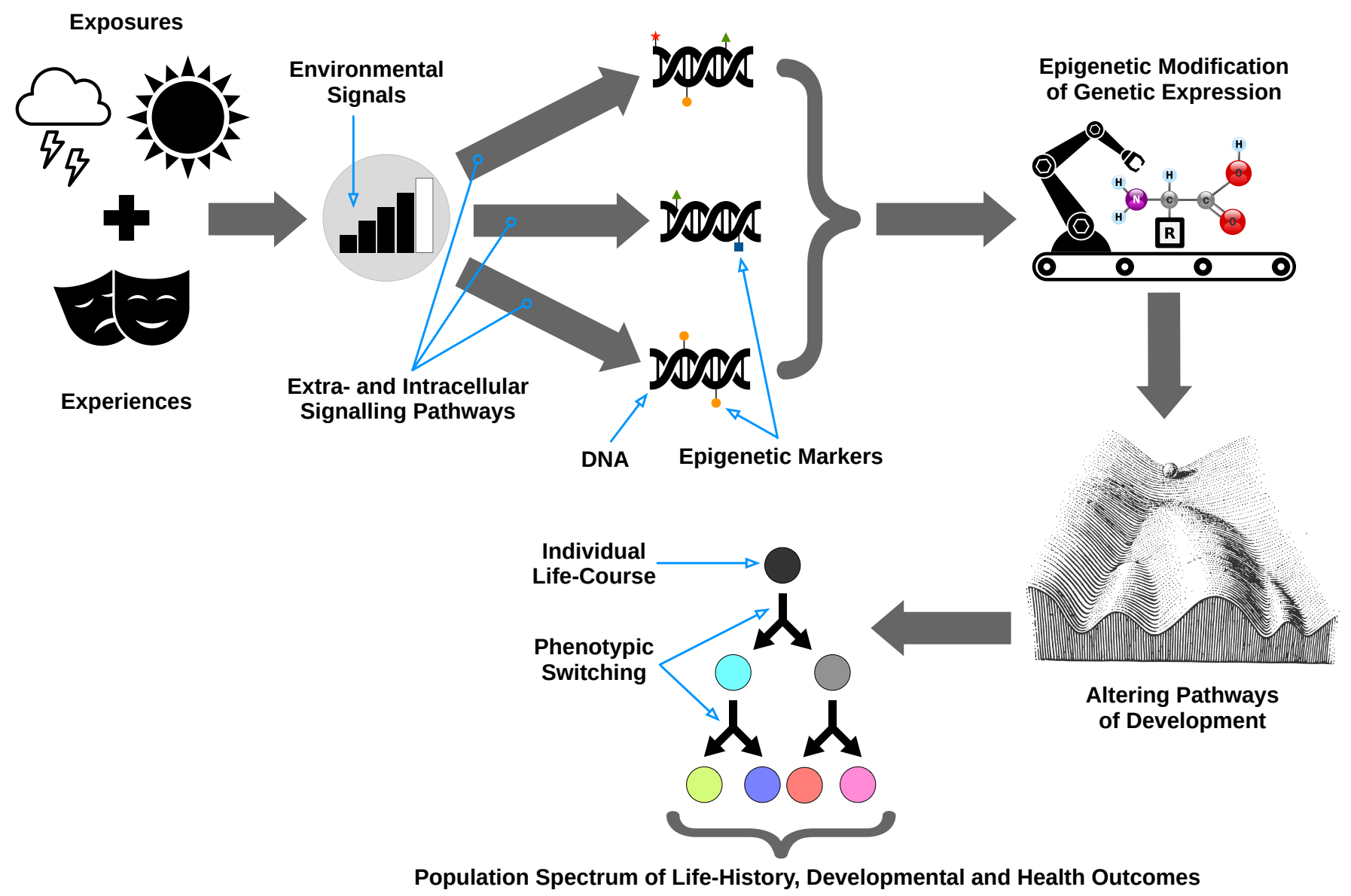

Epigenetic mechanisms serve to diversify genetic functions by regulating modifying and modulating genetic expression - in response to signals from the environment ${ }^{524}$. Epigenetic processes represent a mechanism of network biology where what happens through environmental exposures and in lived experience can be transmitted through somatic signalling pathways to the genes, producing modification of genetic expression, altering developmental pathways and life-history outcomes and driving phenotypic change.

\section{The Interactive Genome and the Plastic Organism}

The quality of parental care has a broad impact on mental health, including the risk for psychopathology. Studies in the rat directly link the maternal care environment to long-term effects on neural systems that regulate stress, emotional function, learning and memory and neuroplasticity. Naturally occurring variations in maternal care in the first week of life in rats are associated with changes in brain and behavior that persist until adulthood. These effects are reversed by cross-fostering, demonstrating a causal link between maternal care and gene expression programming. ${ }^{543}$

Across diverse taxa, the phenotypes (traits and characteristics) of offspring are not strictly determined by their genes but are dependent upon environmental features and conditions. In many species, the relationship of an offspring with its parents exemplifies the interactions between genes, environment, biology and behaviour in the scripting of development. The dynamics of the parental relationship plays a 
profound role in the shaping of offspring development and also mediates the relationship between the offspring and the broader environment. For example, the life-experiences of mothers are known to produce 'maternal effects' - a technical term for how both normal and abnormal variations of maternal experience and maternal expression can change the characteristics (phenotype) and life-course outcomes of the offspring ${ }^{544}$. For example, experiments with dung beetles have demonstrated that the level of maternal investment in offspring (provisioning of dung to developing larva) changes with natural environmental variations such as soil moisture and dung quality, leading to profound changes in offspring phenotypes such as body size, horn length and behaviour ${ }^{545}$.

Maternal effects have been demonstrated across diverse taxa - including plants, insects and animals - and natural variations in maternal care are associated with persistent and profound differences in offspring survival, growth, morphology, physiology, learning and behaviour ${ }^{546}$. Paternal effects on offspring phenotypes have also been demonstrated and mothers and fathers can form regulatory complexes differentially acting on the offspring's phenotype and modifying each other's effects $^{547}$. In mammals, mothers invest intensively in pre- and post-natal care of offspring and bi-parental care is relatively rare. However, even amongst species with limited paternal investments in offspring care - there is also evidence for paternal effects on offspring phenotypes. ${ }^{548}$

Extensive research has revealed a broad epigenetic signature of maternal care written into offspring biology and profoundly and persistently shaping developmental, health and life-course outcomes ${ }^{462,543}$. In mammals, including rodents, primates and humans, maternal behaviour has been demonstrated to be a primary determinant of offspring behaviour and neurodevelopmental outcomes ${ }^{462}$. In rodents, ancestral exposures to toxins and stressful events, in both male and female lineages, are associated with altered neurodevelopmental outcomes in subsequent generations including altered stress responses and fearfulness ${ }^{549}$. In monkeys, abusive behaviour has been shown to be transmitted from mothers to daughters and the experience of early abuse produces persistent changes in offspring behaviour and neurobiology such as increased solitary play and altered neurotransmitter activity. Cross-fostering experiments in both rodents and primates demonstrates that offspring traits such as quality of parental care, abusiveness and fearfulness are determined by maternal behaviour rather than by maternal genetics. In humans, longitudinal studies have demonstrated that the quality of mother-child bonding - known as 'secure-attachment' - is crucial to the child's cognitive, emotional and social development. Children who experience secure attachment demonstrate more self-reliance, self-confidence and self-esteem, are more emotionally regulated, express more positive emotion and behave more flexibly in response to stress than children who experience insecure attachment. Insecure and disorganised attachment is associated with significantly higher risk of psychopathology in later life including dissociative disorders, aggression, conduct disorder and self-abuse. ${ }^{462}$ 
Maternal effects on offspring defensiveness - meaning persistent changes in the offspring's sensitivity and magnitude of reaction to threat - have been widely demonstrated across taxa including plants, animals and insects. Commonly, exposure of the mother to some form of significant adversity or threat is reported to produce persistent forms of defensiveness in the offspring ${ }^{550}$. In studies of vertebrates in both laboratory and wild environments - maternal stress exposures including predator risk, food scarcity, social adversity and adverse weather events are associated with an elevation of maternal circulating glucocorticoids which in turn shape the development of the offspring's hypothalamic-pituitary-adrenal/interrenal (HPA/I) stress-response axis through prenatal biochemical interactions and/or postnatal behavioural interactions ${ }^{546}$. This phenomenon is widely described in the literature as a 'programming' of the stress-response system by the conditions of the early environment - which consequently persistently shapes the adult offspring's patterns of response and behaviour. ${ }^{551}$

\section{Scripting Development}

Pioneering research conducted around the turn of the $21^{\text {st }}$ century established that maternal behaviour can be passed down generations independently of genetic inheritance. In both primates and rodents there are stable individual differences in maternal behaviour that are reliably transmitted from mothers to daughters ${ }^{552}$. For example, populations of rodent mothers demonstrate a normal distribution of stable differences in the frequency of licking and grooming of their newborn pups. ${ }^{550}$ Figure: Idealised normal distribution of maternal licking and grooming of rodent pups

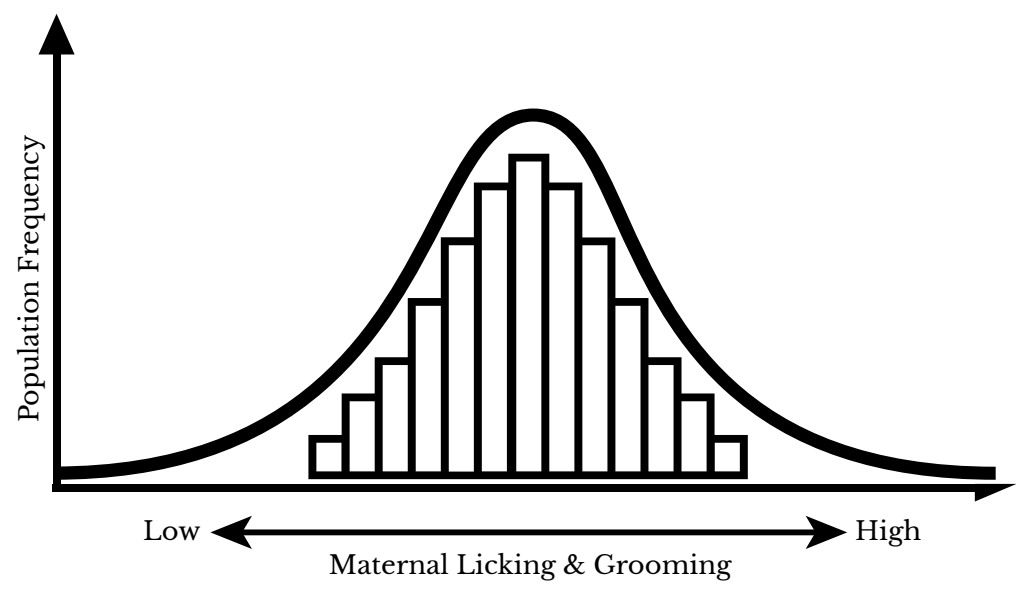

In the Norwegian rat (Rattus norvegicus) maternal contact with pups primarily occurs in the form of a nest-bout in which the mother approaches the litter and gathers her pups under her enabling them to suckle while also licking and grooming them ${ }^{553}$. For the newborn rat, maternal contact including nest-bouts of intensive maternal licking and grooming is vital to both survival and developmental functions including neurodevelopment, thermoregulation, gut development and elimination ${ }^{554-558}$. 
Studies of rats and primates have demonstrated that the early-life rearing environment including the quality of maternal care, regulates the development and function of the HPA stress-response axis of the offspring into adulthood. In rats, maternal behaviour has been identified as a primary determinant of stress-reactivity in juveniles (pups) a characteristic which has been demonstrated to persist throughout the life-course and to even span generations. Experimental manipulation of the rearing environment of rodent pups, by changing the quality of maternal care - produces profound and lasting changes in gene expression, complex neural functions and behaviour ${ }^{559}$.

Many studies have compared the effects on offspring of more frequent licking and grooming mothers (High-LG) to less frequent licking and grooming mothers (LowLG) - comparing mothers whose behaviour falls within 1 standard deviation of the population mean (meaning close to the centre and not at the extremes of the normal range $)^{550}$. The differences in maternal care between High-LG and Low-LG mothers occur only during the first week of the pup's life ${ }^{560}$. During the first week of the pup's life, both High and Low-LG mothers spend the same amount of contact time with their litters and thus the frequency of licking and grooming behaviour is not a function of time in contact with pups. Both High and Low-LG mothers raise a comparable number of pups and there are no observed differences in the weaning weights of the pups - an indicator of adequate levels of maternal feeding in both groups. ${ }^{550}$

Persistent and profound differences in neurophysiology and behaviour are observed in the offspring as a consequence of these normal variations in maternal attentiveness". As adults, the offspring of Low-LG mothers are significantly more fearful, vigilant and anxious, show more heightened HPA axis responses to stressors and more addictive behaviour when offered cocaine or alcohol in experimental environments than offspring of High-LG mothers. The offspring of High-LG mothers demonstrate increased exploratory behaviour in novel environments, decreased startle

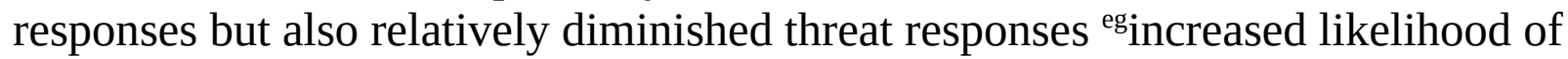
approaching a live predator and less vigilance ${ }^{561-563,560}$. Furthermore, significant differences in brain and neuroendocrine systems are widely reported including in the amygdala, locus ceruleus, nucleus tractus solitarious, hippocampus, frontal and medial prefrontal cortices and the HPA axis. ${ }^{561}$

Given the significance of variations in maternal licking and grooming upon offspring developmental outcomes, even well within the normal range, researchers questioned whether maternal licking and grooming trait is genetically determined - in the words of one of the researchers:

"As geneticists would like you to think, perhaps the mother had the 'bad mother' gene that caused her pups to be stressful, and then it was passed from generation to generation; it's all determined by genetics." 564

- Maternal attentiveness is also experimentally manipulated in many studies by intermittent and gentle human handling of pups in proximity to the mother for a short period of time ( $<15$ minutes) which provokes increased maternal licking and grooming when the pup is returned to the litter - effectively converting Low-LG mothers into High-LG mothers, who then go on to rear High-LG female offspring. 
In order to investigate, researchers conducted a cross-fostering experiment in which a small number of pups from the litter of a High or Low-LG mother were transferred into the litter of another High or Low-LG adoptive mother. Because wholesale transfer of the entire litter is known to alter maternal behaviour, only a small number of pups ${ }^{\mathrm{eg}} 2$ in a litter of 12 were transferred between litters. Controls included the offspring of Low-LG mothers fostered to other Low-LG mothers as well as offspring of High-LG mothers fostered to other High-LG mothers, sham-adoption animals, which were only removed from the nest and then returned back to their biological mothers, and non-manipulated pups of High or Low-LG mothers.

Figure: Patterns of behavioural inheritance from rodent cross-fostering

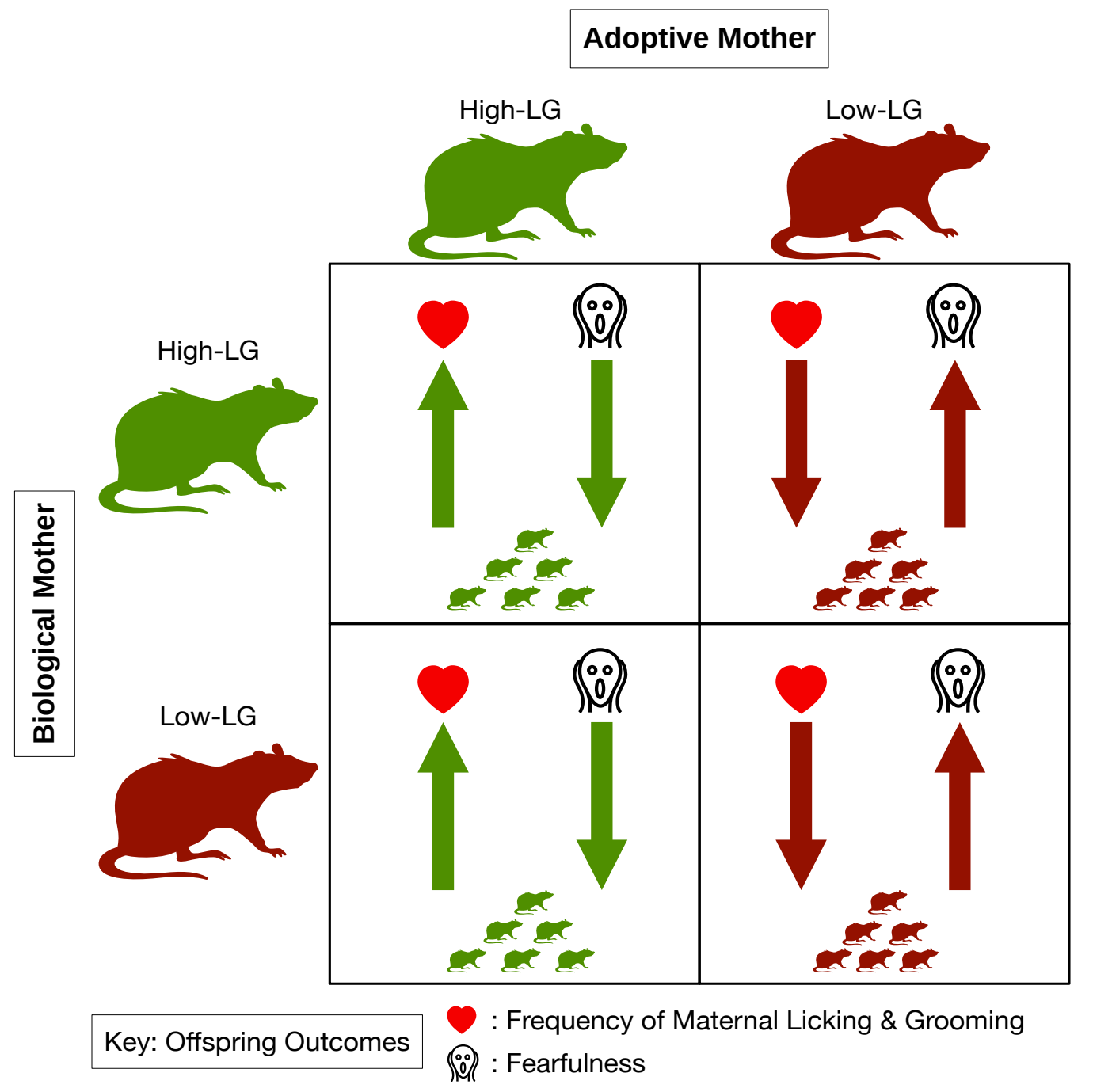

The findings demonstrated that the offspring's phenotypes of fearfulness (in both males and females) and maternal attentiveness (measured by behaviours such as frequency of licking and grooming of pups) were inherited not from the biological mother but rather from the adoptive mother - and that these traits were stable in individuals and could be passed down generations. Female and male biological offspring of Low-LG mothers reared by High-LG mothers were significantly less fearful when exposed to novel conditions than the offspring reared by Low-LG 
mothers and they were also less fearful than the biological offspring of High-LG mothers. Maternal behaviour, studied in a separate subject group, followed the same pattern of inheritance as fearfulness. As adults, the female offspring of Low-LG dams reared by High-LG mothers demonstrated the same frequency of pup licking and grooming as the females born and reared by High-LG mothers. The converse was also true so that females born to High-LG mothers but reared by Low-LG dams demonstrated significantly lower maternal licking and grooming than females reared by High-LG mothers. ${ }^{553}$

Molecular investigation of the adult offspring of High vs Low-LG mothers has revealed stable and persistent epigenetic modifications affecting the expression of the glucocorticoid receptor gene in the hippocampal region of the brain - associated with the regulation of stress responses. Adult offspring who received higher levels of maternal licking and grooming in the first week of life demonstrated increased glucocorticoid receptor expression in the hippocampus, enhanced glucocorticoid feedback sensitivity, decreased corticotrophin releasing factor expression in the hypothalamus and lower levels of hypothalamic-pituitary-adrenal stress response compared to adult offspring who received lower levels of maternal attentiveness. Subsequent studies investigating the effect of early life stress on rats has demonstrated epigenetic modifications producing persistent changes in gene expression at multiple loci in adult survivors including the brain-derived neurotropic factor gene promoter (associated with growth and maintenance of neurons) and the arginine vasopressin gene (associated with fluid regulation in the body and diabetes) ${ }^{524}$.

Genome-wide association studies in rats have demonstrated broadly distributed and coordinated epigenetic modification of genetic expression involving multiple genes and gene-circuits in response to natural variations of maternal care ${ }^{524}$. It appears that epigenetic modification of genetic expression in response to the early environment is widespread throughout the body's systems. Research in primates has demonstrated widespread and organised epigenetic modification in cells of the Prefrontal Cortex of the brain and T lymphocytes (a specialised white blood cell of the immune system) in response to early life adversity linked with poor social development, high reactivity and aggression, increased affinity for alcohol consumption and lower social rank as adults $^{565}$.

Animal research has also demonstrated the reversibility of adverse epigenetic changes. ${ }^{566}$

Figure: Genetically identical offspring (clones) produced by exposing mouse mothers to the environmental pollutant BPA and feeding them either a standard or methyl-rich diet. ${ }^{567}$ 


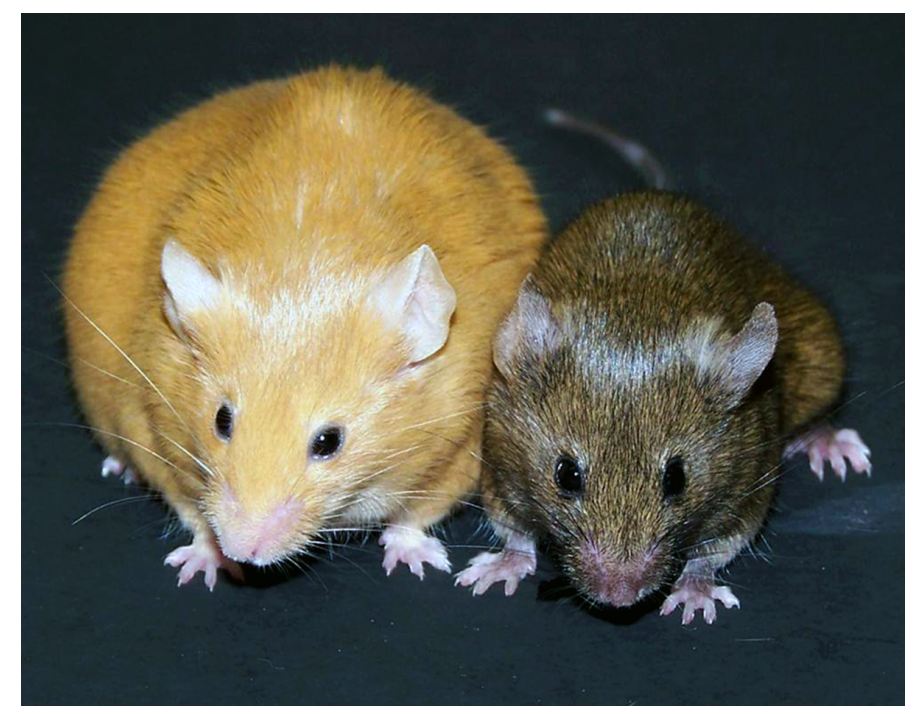

$\mathrm{A}^{\mathrm{vy}}$ mice (pictured) are laboratory bred cloned mice that have a mutation in the Agouti gene - associated with melanin pigmentation distribution in mammals including humans. In the wild, mice with variants of grey, brown or black fur predominate. Mutation of the Agouti gene produces variants with yellow or red hair. Expression of the Agouti mutation produces a pathology called yellow obese syndrome characterised by obesity, metabolic disease and increased rates of cancer ${ }^{566,568,569}$. Importantly, the presence of the genetic mutation alone does not lead to automatic expression of the pathological traits (phenotype) as seen above where both individual clones carry the Agouti mutation and the expression of the genetic mutation depends on environmental exposures.

In $\mathrm{A}^{\mathrm{vy}}$ mice, a diverse range of maternal exposures including low-dose ionising radiation, ethanol and soy isoflavones produce characteristic changes in offspring fur pigmentation by altering Agouti gene expression ${ }^{566}$. Exposure of $\mathrm{A}^{\mathrm{vy}}$ mothers to Biphosphenol-A (BPA) - a ubiquitous environmental contaminant that is used in the manufacture of the lining of mass produced canned food and beverage containers, baby bottles, dental sealants and in large quantities in the thermal paper used in cashregister and ATM receipts - produces offspring with the yellow obese syndrome (pictured). However, when $\mathrm{A}^{\mathrm{vy}}$ mothers were exposed to BPA and fed a special diet (through supplementation with folic acid, betaine, vitamin $\mathrm{B}_{12}$, and choline) the offspring did not manifest the yellow obese syndrome. ${ }^{570}$

In humans, early life adversities are common and drive a broad range of adverse behavioural, psychiatric, immune and metabolic system outcomes ${ }^{571,572}$. Animal and human studies indicate that epigenetic modification of genetic expression represents a probable pathway linking environmental exposures and lived experiences with systemic changes in biological structure and function. Research has demonstrated that maternal adversities such as depression, war-related stress and partner violence during pregnancy produce epigenetic modification in the fetus that is evident in cordblood. Widespread epigenetic changes have been demonstrated in the immune $\mathrm{T}$ cells 
and peripheral blood mononuclear cells of human teenage offspring of mothers exposed to natural disaster whilst pregnant..$^{524,572}$

In summary:

- epigenetic processes are inherent in development across taxa

- epigenetic processes represent (an example of) biological mechanisms enabling experiences and exposures throughout the life-course to get under the skin driving changes in biological structure and function - through the modification of genetic expression

- epigenetic modification of the genome can be systemic, stable and may, in principle, be reversible

- natural variations in environmental conditions such as levels of maternal care in the early environment alter genetic expression via epigenetic signalling pathways and can produce profound and persistent changes in offspring characteristics (phenotypes)

- it appears likely that the widespread and organised epigenetic modification of the genome in response to normal and abnormal environmental exposures and experiences is a primary driver of differences in life-course and health outcomes between individuals across diverse taxa.

\section{Information and Evolution}

Evolutionary theory is itself evolving - from the gene-centric model of the 20th century Modern Synthesis to an information-centric model in the 21st century. The Modern Synthesis held that natural selection operates on and organises genes and genotypes. But in the 21st century Post-Genomic Synthesis, it is recognised that selection does not act on genes but on what is expressed in biological structure, function and behaviour - which is the organism's phenotype. And the organism's phenotype is a product not of direct genetic dictation but of an ongoing conversation between the organism and the environment - via biological signalling networks.

In evolution, information from multiple dimensions of the environment organises the organism's structure and function across generations. Multiple interacting levels of information organise the expression of the organism - its phenotype. Each level is considered to emerge as a self-organising outcome of the levels that precede it and operations at each level are understood to alter biological structure and function.

\begin{tabular}{|l|l|l|}
\hline \multicolumn{1}{|c|}{ Information Type } & \multicolumn{1}{|c|}{ Source } & \multicolumn{1}{c|}{ Vector of Transmission } \\
\hline Genetic & Ancestral environment & DNA \\
\hline Epigenetic & Contemporary environment & Exposures and Experiences \\
\hline Behavioural & Social environment & Learning and Conditioning \\
\hline
\end{tabular}

In modelling the organism as a complex adaptive system - neither genes nor the environment are considered to specify or dictate the organism's phenotype but rather environmental features and perturbations can provoke and drive self-organising changes in the organism's function and structure. 


\begin{tabular}{|l|l|l|}
\hline Cultural & Cultural environment & Ideas and Worldviews \\
\hline Ecological & Global environment & Overview Effects \\
\hline
\end{tabular}

Firstly, organisms are organised by genetic information which has evolved through interactions with ancestral environments - for example properties of the physical world such as aerodynamics and optics are 'embodied' in the structure and function of a bird's wings and its eyes which arise from information encoded in homeobox Hox and Pax genes. Secondly, organisms are organised by epigenetic information derived from generational and individual life-histories - exposures and experiences that shape development - for example the plasticity of the timing of metamorphosis in amphibian species in response to environmental conditions involves the triggering of epigenetic mechanisms which regulate the pace of development. Thirdly and most obviously in organisms with complex nervous systems and extended early development - the organism's development is shaped by behavioural information from kin and community - for example the differential development of the HPA stress-response axises of rats exposed to High-LG vs Low-LG mothers - which produces life-long and intergenerational consequences. Fourthly, organisms such as ourselves are organised by cultural information - symbols and ideas - which give rise to civilisations and their histories. ${ }^{573-575}$

A fifth evolutionary level appears to be emerging - in which systemic information acquired through feedbacks from the global environment is organising human expression. The Overview Effect was originally described as a common response in astronauts to the sight of the Earth from Space - triggering overwhelming emotion and profoundly changing the sense-of-self through identification with humankind, life and planet - called self-transcendence.

For example - as described in these reports from a North American astronaut:

You identify with Houston and then you identify with Los Angeles and Phoenix and New Orleans....and that whole process of what it is you identify with begins to shift when you go around the Earth...you look down and see the surface of that globe you've lived on all this time, and you know all those people down there and they are like you, they are you — and somehow you represent them. You are up there as the sensing element, that point out on the end...you recognize that you're a piece of this total life.

and from a Russian astronaut:

The feeling of unity is not simply an observation. With it comes a strong sense of compassion and concern for the state of our planet and the effect humans are having on it. It isn't important in which sea or lake you observe a slick of pollution or in the forests of which country a fire breaks out, or on which continent a hurricane arises. You are standing guard over the whole of our Earth. $^{.76}$ 
Intensely self-transcendent experiences are historically associated with meditation, prayer, religious and shamanic practices, near-death experiences and psychedelic drugs - and are associated with both short and long term effects including increased vitality, positive affect, compassion, decreased anxiety and reorientations in values, world-view and self-concept. ${ }^{577-579}$

Self-transcendence is also associated with exposure to adversity. Although adverse psychological outcomes (such as depression and post-traumatic stress injury) are frequently reported as consequences of exposure to severe adversity, broad psychological growth in areas such as relationships, agency, orientation to life and spirituality is also historically reported as a common (but not universal) response to adversities including earthquakes, maritime disasters, natural disasters and war and is contemporarily reported in response to a wide range of adversities including highly challenging life events, war and terrorism, accidents, sexual assault, bereavement, HIV infection, myocardial infarction, bone marrow transplantation, cancer and chronic illness. As many as $70 \%$ of survivors of various forms of trauma report positive change in at least one domain of life ${ }^{580-583}$. In particular, self-transcendence has been identified as one of the key personal characteristics enabling survival in disasters and subsequent recovery ${ }^{584,585}$.

Self-transcendence has been described as a higher form of human psychological development. In his later work, the eminent psychologist Abraham Maslow reconsidered his hierarchy of human needs and asserted that self-transcendence constitutes a level of human development beyond self-actualisation:

Transcendence refers to the very highest and most inclusive or holistic levels of human consciousness, behaving and relating, as ends rather than means, to one self, to significant others, to human beings in general, to other species, to nature, and to the cosmos. ${ }^{586}$

And the Holocaust survivor and eminent psychiatrist Viktor Frankl considered that human development culminates in self-transcendence with self-actualisation a secondary outcome:

The more one forgets himself - by giving himself to a cause to serve or another person to love- the more human he is and the more he actualizes himself. What is called self-actualization is not an attainable aim at all, for the simple reason that the more one would strive for it, the more he would miss it. In other words, self-actualization is possible only as a side-effect of self-transcendence. ${ }^{577}$

Cross-cultural studies indicate that self-transcendence is a stable individual characteristic that is distinct from the classically recognised 'Big 5' personality traits ${ }^{\psi}$. Self-transcendence has been identified as a spontaneous outcome of so-called 'successful aging' (meaning continuous adult development associated with positive life-satisfaction) and has features including decreasing reliance on social definitions

$\psi \quad$ The Big 5 are Openness, Conscientiousness, Extroversion, Agreeableness and Neuroticism ${ }^{587}$ 
of self, increasing interiority, and a greater sense of connectedness with past and future generations. ${ }^{577,588-590}$

I used to feel that I was out on a river being carried away by the stream without being able to control it. Even if I wanted to go ashore, I couldn't control it; I was carried away both from pleasant and unpleasant things. But today I feel like the river. I feel like I'm the river. I feel that I'm part of the flow that contains both the pleasant and the unpleasant things. ${ }^{589}$

Research in aging (Gerontology) has progressed from a misery model - focusing on progressive loss of function, deterioration and disengagement from life with advancing age, to a success model focusing on the preservation and maintenance of function - measured in terms of freedom from disease and disability, high cognitive and physical abilities and active engagement. However, neither of these models fit the cross-cultural, longitudinal and self-reported evidence, for example - which consistently demonstrate that while only a small minority of elderly people fit the 'objective' research criteria for successfully aging, the majority of elderly people report that they have good quality of life and are aging well. Consequently, an ecological model of aging has arisen which:

- represents a shift from a biomedical fixation on physiological criteria to encompass broader biopsychosocial, evolutionary-developmental and lifehistory concepts of aging

- balances objective (researcher centric) and subjective (elderly centric) criteria and definitions of aging well

- distinguishes different cultural definitions and models of aging well - for example those which prioritise individual independence and ability or those which emphasise social and spiritual connectedness

- recognises important developmental tasks of aging such as positive adaptation (resilience), the emergence of wisdom and self-transcendence that may provide adaptive advantages not simply to the individual but to the community. ${ }^{588,589,591-593}$

In the ecological model of aging, self-transcendence, wisdom and resilience are considered to be higher or more advanced functions of human development. Importantly, in regards to wisdom for example - many studies have been done which demonstrate that there is little or no direct association between age and the development of wisdom - in other words wisdom (and likely self-transcendence and resilience also) is not a function of age but rather is a function of experience involving exposure to and problem-solving in diverse/adverse conditions. ${ }^{591}$

In global context, ecological feedbacks arising from intensify human alteration of ecosystems and environments may contribute to an increasing occurrence of self- 
transcendence in populations. For example, the frequency of natural disasters ${ }^{\Omega}$ dramatically increased in the latter half of the $20^{\text {th }}$ century:

Figure: Number of Natural Disasters 1900-2011 594

600

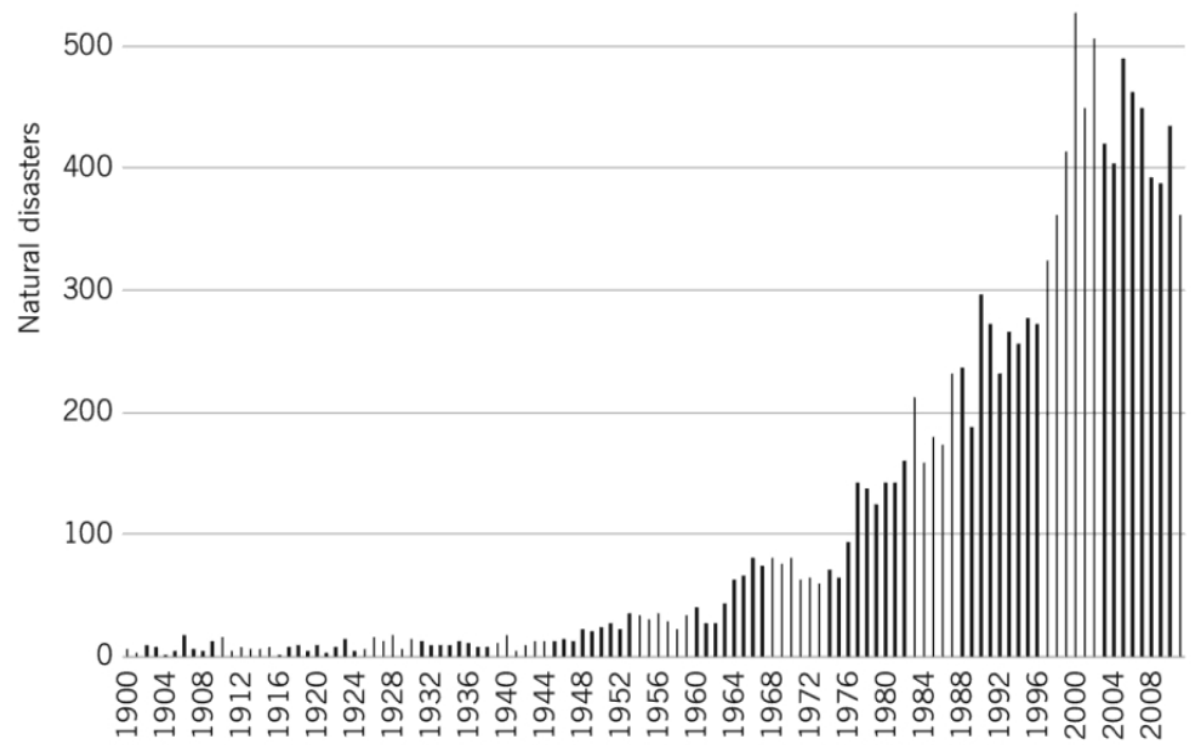

Although natural disasters cause only a small fraction of total global mortality (between 0.01-0.4\% annually) with significant decline in total fatalities between 1960 to 2019, they inflict significant human impacts measured by the numbers of people affected (including injured, requiring basic necessities and medical assistance and rendered homeless) and the global economic costs - both of which have substantially increased. 595

Across disciplines, researchers now consider that the term 'natural disaster' is itself a misnomer - because although these phenomena are 'forced' by diverse environmental events they produce characteristic patterns of social outcomes. A wildfire, flood or earthquake that doesn't affect human life and property is typically not thought of as a disaster. A natural disaster is the outcome of a collision between geophysical processes and human social systems. The impacts of disaster and the patterns of recovery are not simply determined by the magnitude of natural hazards but by the structure of society - particularly patterns of social inequality. For example, since 2000, 99.7\% of counties in the US have experienced significant impacts from environmental disasters with an average of 5 events per year over the last 50 years with escalating costs predicted. In 2015 alone, U.S. insurance companies and the Federal Emergency Management Agency (FEMA) distributed \$30 billion in recovery funds to regions affected by natural disasters. Longitudinal research using a nationally representative sample in the US has demonstrated that over a 14 year study period - following environmental disasters, white middle-class residents not only

$\Omega \quad$ Natural disasters are technically defined as adverse environmental events (such as tornadoes, earthquakes, floods and wildfires) which meet one or more of the following criteria: ten or more people reported killed, one hundred or more people reported affected, declaration of a state of emergency and/or call for international assistance. ${ }^{594}$ 
recover but significantly profit from disaster in terms of increasing wealth while black and latino residents tend to lose wealth, jobs and homes. Moreover, the more government aid a region receives post-disaster, the more wealth inequality becomes polarised. ${ }^{596-599}$

Figure: Global population affected by all natural disasters 1900-2019 ${ }^{595}$

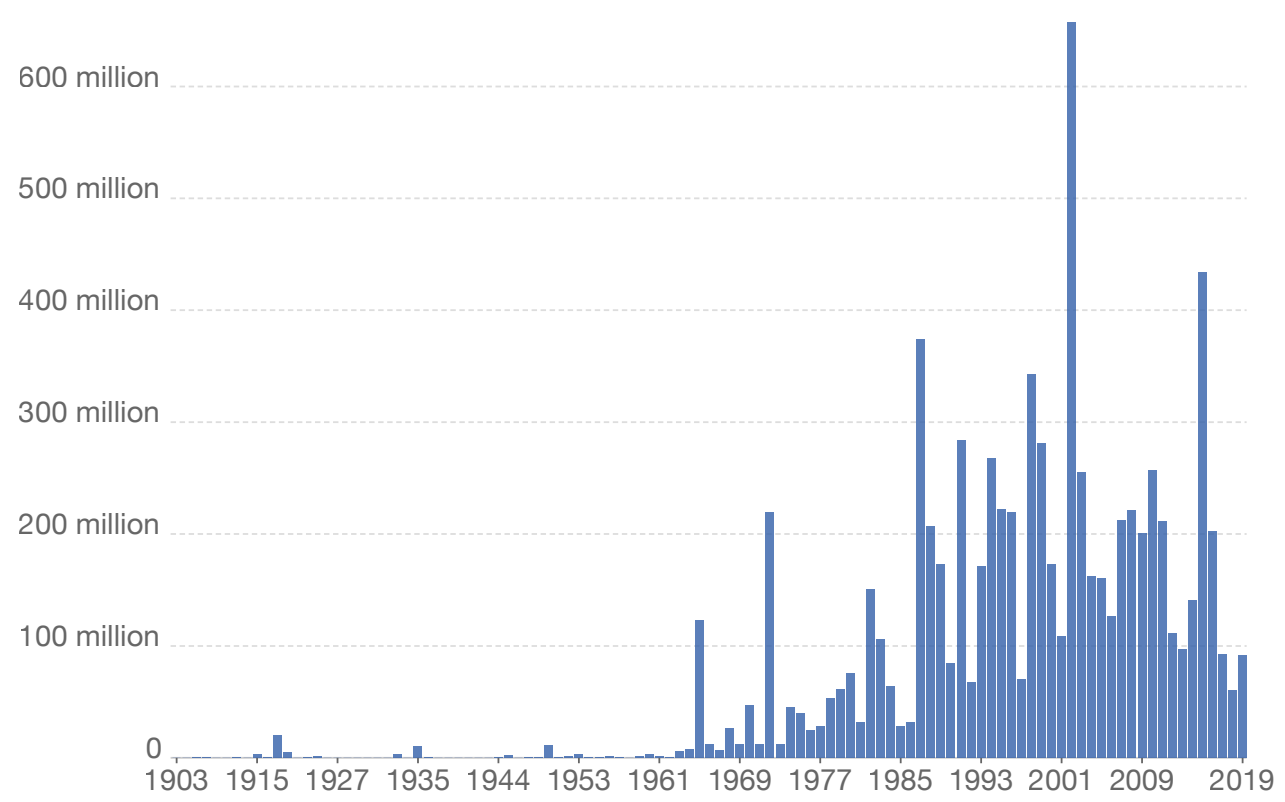

Figure: Global damage costs from all natural disasters 1980-2019595

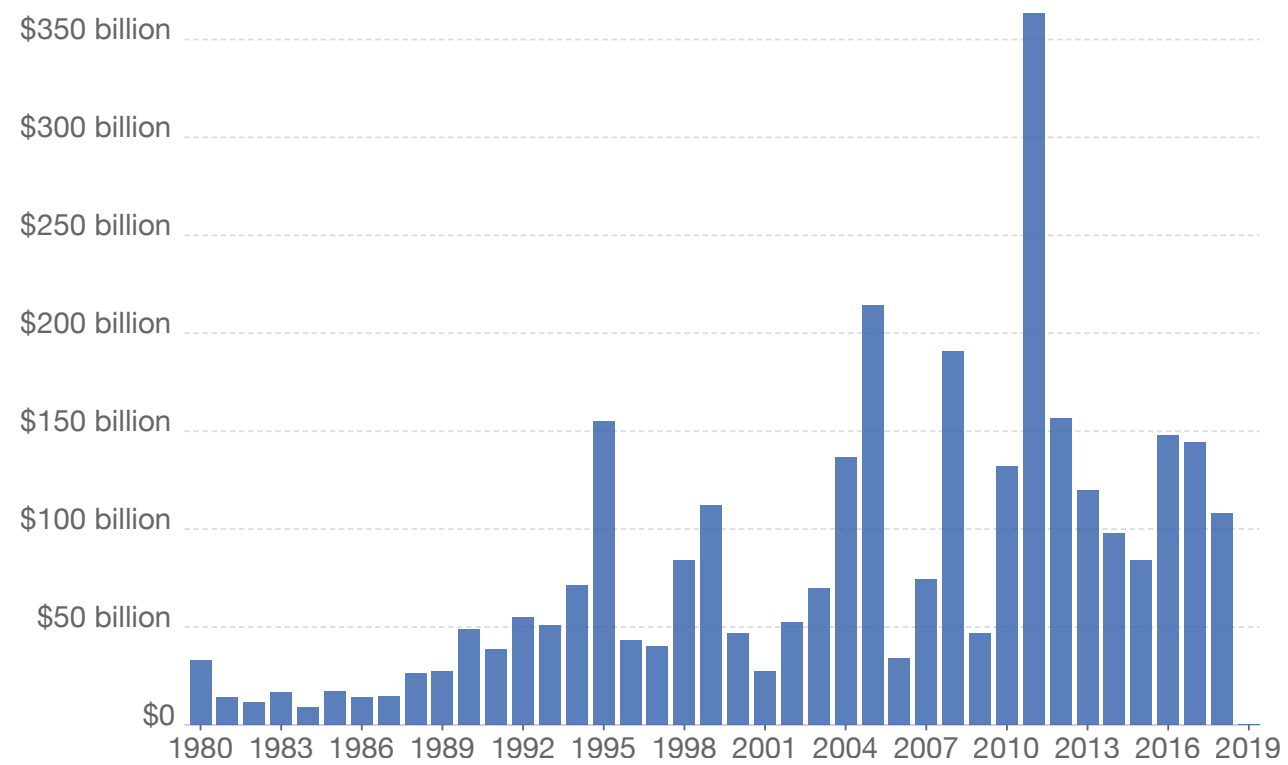

The phenomenon of modern megafires represents a key example of ecological feedback associated with human manipulation of ecosystems. Fire is a fundamental process in Earth's systems connecting ecosystems, biogeochemical cycles and climate and a primary driver of species biodiversity. Currently, around $4 \%$ of the global land mass undergoes burning annually. Wildfire arose with the emergence of the first terrestrial plants in the Silurian period and has shaped the Earth's climate and 
biosphere for around 400-350 million years. Thus humans have always coexisted with wildfire. ${ }^{600-602}$

The functioning of natural ecosystems produces 'ecosystem-services' which are essential to the survival and success of myriad living systems. Recurrent wildfires, as a natural ecosystem function, produce many ecosystem services that support human life.

Table: Ecosystem services provided by recurrent wildfires to early and contemporary human life $e^{603}$

\begin{tabular}{|l|l|}
\hline \multicolumn{1}{|c|}{ Type } & \multicolumn{1}{c|}{ Service } \\
\hline Provisioning & Producing open spaces for pastures, agriculture, and hunting \\
\hline & $\begin{array}{l}\text { Stimulate germination of desirable annual plant species } \\
\text { post-fire }\end{array}$ \\
\hline Regulating & Provide essences, medicines, craft materials \\
\hline & Primary driver of biodiversity \\
\hline & Control of pests affecting humans and livestock \\
\hline & Reduce catastrophic wildfires \\
\hline & Accelerate species replacement under changing conditions \\
\hline & Enhance flowering and pollinator activity \\
\hline Cultural & Water, Oxygen \& Carbon cycles \\
\hline & Spiritual-Ecological \\
\hline & Ecotourism in open ecosystems \\
\hline & Scientific knowledge about the origin of biodiversity \\
\hline & Information about ancestral fire management techniques \\
\hline
\end{tabular}

\section{The Ideal and the Optimal}

One of the earliest and thorniest problems in philosophy and science is categorisation - what makes some things the same and what makes other things different? When we survey populations of similar things - from particles to organisms to galaxies in the universe - we encounter shared individual characteristics (commonality) and individual variations (diversity). One of the classical solutions to the categorisation problem in Western philosophy is essentialism - which holds that things categorised as being of the same kind - from molecules to people and planets - have, at some level, the same intrinsic 'nature' - whether that nature is that they are constituted of the same elements or that they are variations of a common prototypal form. 
Essentialist concepts have produced triumphs in the basic sciences such as chemistry and physics - for example leading to the categorisation and organisation of the atomic elements. ${ }^{604,605}$

Figure: the Periodic Table of Elements ${ }^{606}$

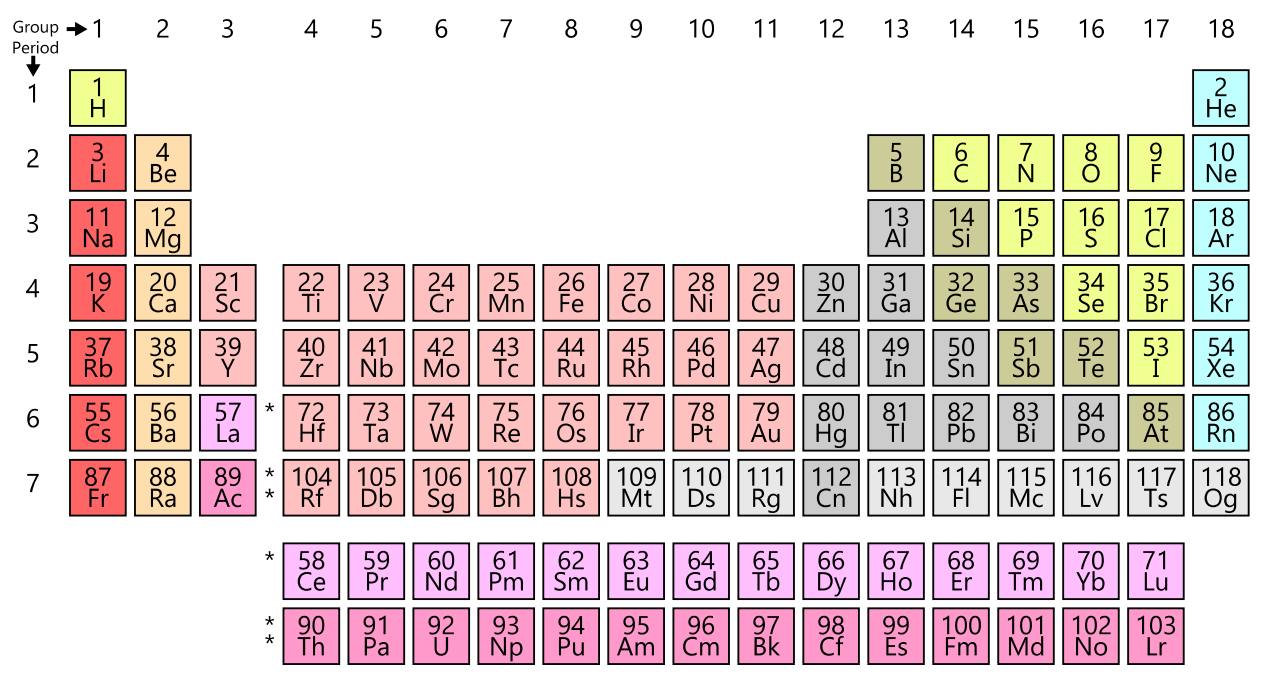

Essentialism - as a model of reality - serves to psychologically stabilise the dynamic nature of the world and the things in it by creating fixed constructs - idealisations of form and function. Essentialist ideas are pervasive in western cultural discourse reflected in beliefs and claims about what is 'right', 'proper' and 'normal' about the world around us. In the life-sciences essentialism appears as an implicit belief in biological ideals - that under ideal environmental conditions, an organism follows an ideal developmental trajectory into the ideal form for its species and that in less-thanideal conditions - imperfections/flaws in development, form and function follow manifesting as disease, disorder and dysfunction. In $20^{\text {th }}$ century biology, essentialism became translated into a search for 'species-typical' traits and characteristics which were used to define the 'normality' of individuals in a population in contrast to lessfrequent traits and characteristics which were described as 'abnormal' ${ }^{24}$. Similarly, in the Modern Synthesis of evolutionary theory - the information encoded in genes became represented as the pure evolutionary 'signal'- which defined the species, whilst developmental and phenotypic variability became represented as a kind of impurity or 'noise'. 392

But essentialism and its constructs of stable and distinct ideal types, normality and proper forms and patterns - are confounded by certain characteristics of commonplace complex systems. Many diverse complex systems such as national and global economies, cities, the internet, ecosystems, organisations and organisms which are not composed of the same constituent elements - are nevertheless not merely metaphorically but are substantively alike - demonstrating critical features in common such as evolution, development, adaptation, self-organisation and emergence. 
Complex adaptive systems including organisms and ecosystems are characterised by undergoing self-organising change - continuously and inexorably becoming something else - transforming - through interactions with other systems in the world around them. Complex systems are precariously poised 'at the edge of chaos', sensitive to perturbations from other systems and open to disruption. Complex systems feature discontinuous and abrupt patterns of radical change. For example a basic feature of a natural environmental system such as a forest or a coral reef is that it's moment-to-moment state is not one of stability and equilibrium but rather is critically poised far-from-equilibrium.

there is no such thing as 'nature's balance', no real or primordial nature that would be in equilibrium if only humans had not intruded. The effects of humans are subtly and irreversibly woven into the very evolution of landscape...

Ecological systems are on the edge of chaos without a 'natural' tendency towards equilibrium, even if all humans were to depart forever from the scene. Indeed, many ecological systems themselves depend not upon stable relationships but upon massive intrusions, of extraordinary flows of species from other parts of the globe and of fire, lightning, hurricanes, high winds, ice storms, flash floods, frosts, earthquakes and so on. The 'normal' state of nature is thus not one of balance and repose; the normal state is to be recovering from the last disaster. ${ }^{607}$

The weight of evidence from the biological record demonstrates that evolution does not pursue or produce perfection - or ideal fixed types of form and function, but rather, produces dynamic optimality - plasticity and variability of form and function within individuals and across populations as a consequence of contending with volatility, uncertainty, complexity and adversity in ancestral and contemporary environments.

For example, in human evolution - volatility, uncertainty, complexity and adversity have frequently characterised ancient ancestral environments - exerting selection pressures which have shaped our emergence as a species and patterned our development. Dramatic climate oscillations have characterised the late Cenozoic era (from about 65 million years ago to the present), significantly escalating over the last 6 million years and likely a primary driving force of the evolutionary branching and emergence of hominid species including Homo sapiens ${ }^{608-610}$. A long-held orthodoxy in paleoanthropology until the late $20^{\text {th }}$ century has held that the gradual climate change occurring over this period - the cooling and drying of the Earth - led to 'the forest leaving the apes' and produced increasingly arid savannah in East Africa driving the development of hominid bipedalism, more complex social organisation and ultimately migration ${ }^{611}$. However, recent research has unearthed fossil molecular evidence (such as soil lipid residues from the breakdown of ancient plant species) dating to a period between 2 to 1.8 million years ago in Olduvai Gorge in northern Tanzania, which reveals the coexistence of both continuous (gradual) and abrupt (discontinuous) climate change - producing radical and rapid flipping of the 
environment between open grasslands (savannah) and closed forests in repeated cycles of not millions of years but mere hundreds to a few thousands of years.

Unstable and rapidly shifting environments compel inhabitant species to adapt to variable ecosystems - for example by diversifying their dietary range - which is strongly linked in primates to increasing brain size (encephalisation) - leading to the hypothesis that extreme climate variability has been a critical evolutionary driver in the evolution of our species. ${ }^{612}$

This period around 2 million years ago saw the emergence and dispersal of Homo erectus - a species which has been described as a turning point in hominid evolution - characterised by a constellation of adaptive features contributing to a greater flexibility to explore and exploit opportunities in novel environments and to broaden the range of livable habitats - including a significant increase in brain size, changes in life history (pattern of development) such as shortened inter-birth intervals and slower maturation (likely indicating increasing enculturation), changes in pelvic morphology to accommodate increased cranial size at birth, changes in body size and gender dimorphism, changes in shoulder morphology allowing throwing of projectiles, adaptations for long-distance running and more complex social behaviour. Overall, it is apparent that hominid evolution has been driven and shaped by complex dynamics including both gradual and abrupt climate change, environmental instability and oscillations and competition and interactions with other evolving species. ${ }^{612-614,611,608}$

Even during periods when the natural environment has been relatively stable, adversity has persisted as a defining feature of human existence. For example - it appears likely that the principal threat to ancestral infant and child survival was not from predatory animals but rather from constraints upon parents making them unable or unwilling to invest in child survival. ${ }^{24}$

One universal in the history of childhood stands above all others. The history of childhood is a history of death. Before modernized civilizations, across all times and cultures, children faced grim odds of survival...From the pre-Columbian Americas, to Ancient Rome, to medieval Japan, to the European Renaissance, roughly a quarter of infants died before their first birthdays and half failed to survive to adulthood. ${ }^{407}$

Infant and child mortality rates (the likelihood of dying before the ages of 1 or of 15yrs, respectively) in historical cultures widely separated by geography, culture and time - demonstrate striking consistency - with an average infant mortality rate of about $27 \%$ and a child mortality rate of about $46 \%$, rates which are consistent with data from diverse contemporary hunter-gatherer populations. In contrast in very recent history, demographic transitions have produced a 50-fold increase in child survival and globally - overall mortality rates have decreased 5-fold since the 1950s. ${ }^{407,615}$ 
Thus, according to scientific evidence, living organisms have not evolved in stable and secure environments akin to the mythic Garden of Eden but in ancestral environments characterised by volatility, uncertainty, complexity and adversity. Complex and dynamic environments prohibit any universal solution to the problem of biologically adaptive structure and function - and therefore can be no one right, proper or 'natural' form for an organism to take nor path for its development to follow. Instead, species and populations which are confronted by complex and dynamic environments, adapt by exploring plural developmental pathways producing phenotypic plasticity and variability - because what is adaptive and optimal for individuals in one set of conditions becomes suboptimal or even fatal when conditions change.

The phenomenon of play behaviour in animals demonstrates how organisms evolve towards optimality not perfectibility - enabling local adaptability through mechanisms such as developmental plasticity and population variability - rather than towards ideal types of form and function.

Play has been technically defined:

Play is repeated, seemingly non-serious behavior differing from more adaptive versions structurally, contextually, or developmentally, and 'voluntarily' initiated when the animal is in a relaxed, unstimulating, or low stress setting. ${ }^{.16}$

Play is observed in diverse animal kinds including mammals, marsupials, reptiles, birds, fish, molluscs, spiders and insects. Play is a basic developmental function that is one that appears early in development - for example in horses, foals have been observed playing within 2 hours of birth. ${ }^{617}$

Play has been mythologised in both popular and academic literature - elevated to the stature of a quintessential element - a defining feature of what makes us human.

Now in myth and ritual the great instinctive forces of civilized life have their origin: law and order, commerce and profit, craft and art, poetry, wisdom, and science. All are rooted in the primaeval soil of play. ${ }^{617}$

and

Of all animal species, humans are the biggest players of all. We are built to play and built through play. When we play, we are engaged in the purest expression of our humanity, the truest expression of our individuality. Is it any wonder that often the times we feel most alive, those that make up our best memories, are moments of play? ${ }^{618}$ 
By contrast, the evolutionary evidence indicates that play is neither a 'normal' nor essential feature of development but is rather a plastic and conditional feature of development. The presence or absence and quality of play represent a plurality of adaptive developmental pathways in many species optimising for survival in variable and uncertain environments.
Play has few immediate benefits and quite high costs in that it uses precious calories that are often hard to come by. However, play can have considerable future benefits: it increases neural interconnectivity and animals that play as juveniles tend to be more adaptable and flexible as adults. They are more motivated to explore their environments, better able to switch between different behaviour patterns and have a greater capacity to reverse previous learning and engage in new learning. Animals that are in good condition play more. That makes evolutionary sense because youngsters who have plentiful resources are likely to have a relatively long life, and therefore they have a great capacity to benefit in the future from the delayed effects of play. In contrast, animals in poor condition play less. This, too, is evolutionarily prudent. When resources are scarce, it makes little sense to use precious calories to fuel play, when that might leave an animal malnourished and without the energy needed to fight disease or to grow. Additionally, animals that are in a poor condition are less likely to survive long enough to reap the long-term benefits of play. In short, from a modern evolutionary perspective, there is no one ideal pattern of play - what is optimal depends on the environment in which the individual is living, and the trade-offs necessary to survive and reproduce in that environment. ${ }^{24}$

The attenuation of play development is thus part of and represents a phenotypic shift - a pattern of system-wide change that evolved as an adaptive response to environmental conditions - involving a reorientation of metabolic and physiological activity away from investment in somatic and cognitive development into a mode of life (life-history pattern) which is optimised for survival in adversity and likely follows an accelerated developmental time-table. This altered developmental trajectory changes neurophysiological and behavioural development and may produce biobehavioural outcomes (for example common psychological conditions such as depression, addiction and anxiety $)^{619-621}$ which are subsequently characterised and diagnosed as pathologies but which actually originate in biological adaptation not biological failure.

In an orthodox or classical model of child development, the presence of play in children is normalised (assumed to be the 'natural' pattern of development) and the absence of play is modelled as a kind of deficiency and pathology. The fixation on the individual and their circumstances and the failure to integrate an evolutionary framework, results in a deficiency model which fails to recognise an adaptive function because its benefit is not evident at the individual level but rather at the population level. Deficiency modelling frames playless (vs playful) children in terms of supposed breakdowns in 'normal' or 'proper' structure, function and development and may then logically seek to rectify what is presumed to be broken by targeting the 
individual with multiple interventions at nutritional, familial, socioeconomic, scholastic or psychological levels in order to restore 'normality' - presumed to be a proper course of childhood development. All of which may produce profound individual benefits.

Critically, however - the therapeutic fixation on individuals abstracted from evolutionary, developmental and social contexts reproduces the problems of $3^{\text {rd }}$ and $4^{\text {th }}$ wave health modelling - and typically fails to address the upstream determinants driving increasing social inequalities and health disparities in populations and globally. In parallel, the treatment and cost-ineffectiveness of current biomedical interventions for the major classes of NCD's and common psychological conditions, when measured at the population level, is an outcome of a broad failure of modelling and policy to address the upstream systemic determinants of health. ${ }^{622-626}$

In contrast, an evolutionary-developmental model of physical and mental (or whole systems) health recognises that species contending with volatile environments do not evolve towards perfect or ideal phenotypes but towards flexibility and optimality. Species evolve plural developmental trajectories in response to the 'predictable unpredictability' of dynamic environments. Thus, an evolutionary-developmental modelling presents play on a spectrum of developmental pathways. The absence of play in children is not represented as a deficiency or pathology, but more commonly as an adaptive response to environmental conditions. Instead of focusing on fixing and correcting brokenness in individuals, systemic interventions focus on creating different developmental contexts which support a phenotypic shift from a biological system optimising for survival in adversity to a biological system optimising for recovery, growth. As such this represents $5^{\text {th }}$ wave health modelling - focusing on systems and centering the primary determinants of health and disease - the upstream social causes.

In summary - organisms which have evolved in contention with volatile and uncertain ancestral environments do not evolve towards a single universal 'ideal' type but towards what is optimal whilst conserving variability to contend with predictably unpredictable environmental change. Developmental plasticity appears to be universal across living taxa indicating that life-course plasticity is a basic adaptation to environmental complexity, volatility, uncertainty and adversity. Populations and species exposed to such ancestral environments demonstrate a plurality of developmental pathways manifesting in normative phenotypic diversity rather than phenotypic normality. 


\section{Part Five: The FEAR Model}

Recent work has put forward the hypothesis that we cannot find central disease mechanisms for mental disorders because no such mechanisms exist. ${ }^{627}$

Convergent lines of evidence across disciplines indicates that common psychological conditions (so-called 'mental illnesses') are not primarily mental phenomena but rather embodied phenomena - outcomes, products and features of adaptive biological responses to environmental complexity.

The Features of Evolved Adaptive Responses model proposes that common psychological conditions are outcomes of evolved adaptive responses to environmental conditions enacted through mechanisms occurring at the individual level (life-course) and at levels superordinate to the individual (across populations and generations).

Figure: an Evo-Devo model of the drivers of common psychological conditions

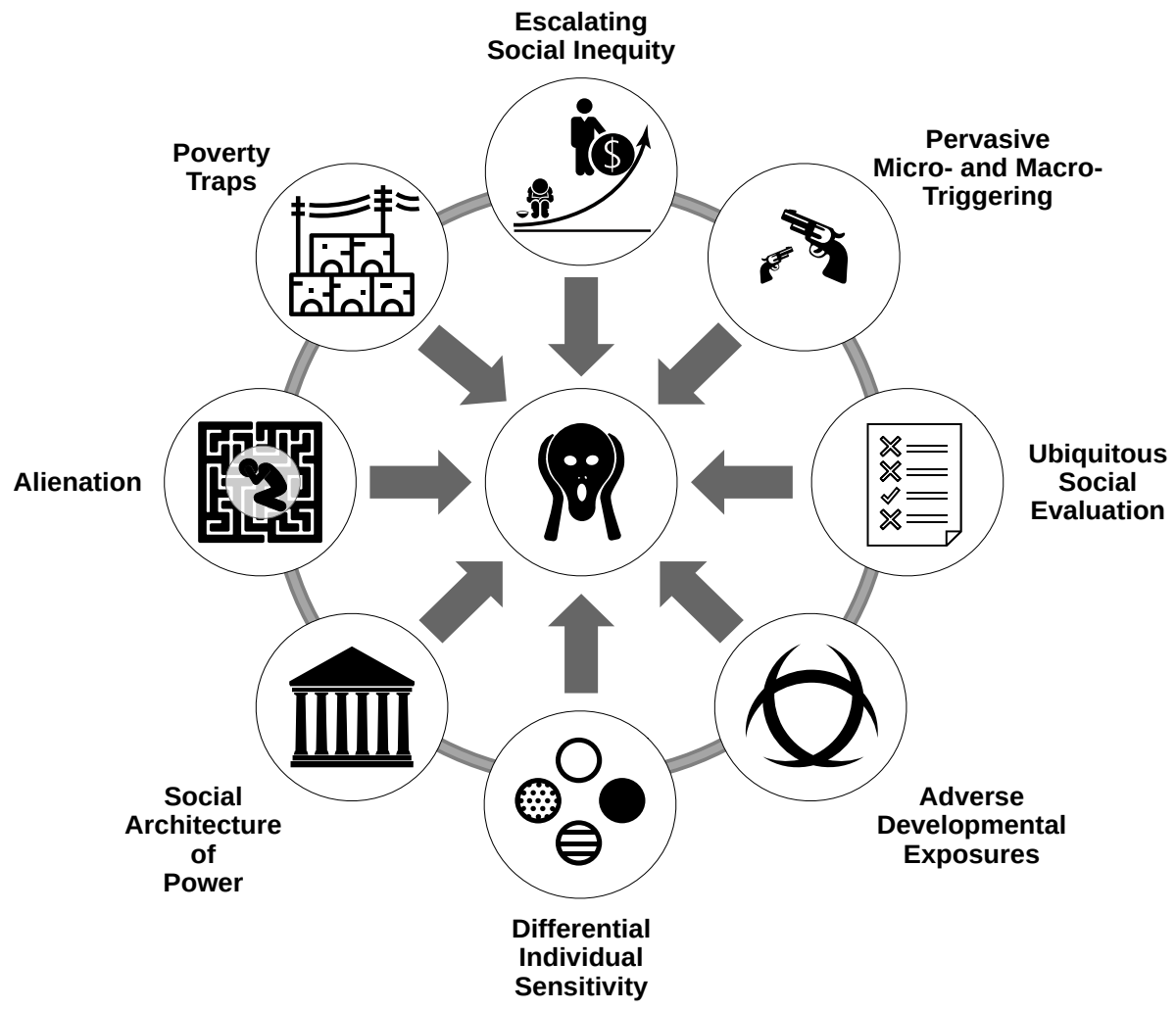

According to an evolutionary-developmental model - common psychological conditions (including traumatic conditions, depression, anxiety, bipolar and psychoses) are outcomes of evolved adaptive mechanisms which are basic to living organisms - such as intra-individual developmental plasticity and inter-individual phenotypic diversity. 
In order to survive and flourish, species must contend with environments characterised by features including volatility, uncertainty, unpredictability, complexity and exposure to adversity and advantage. In such environments, there can be no ideal form and function - but rather only what is optimal for a current range of conditions. Thus species must balance adaptation to current conditions with adaptability for the likelihood of environmental change. Species confronted by complex dynamic conditions mitigate against the risks of maladaptation by producing diversity rather than uniformity of phenotypes within populations. Across taxa, species demonstrate intra-individual plasticity of form and function and interindividual diversity of form and function as mechanisms of adaptive flexibility in the face of change.

An evolutionary-developmental model of common psychological conditions proposes that these conditions have a biological basis, but in contrast to the biomedical model of mental illness, they originate in developmental plasticity - evolved adaptive responses - rather than in forms of biological failure (such as dysregulation, dysfunction, disorder and disease). Critically, because evolutionary processes are organised around the survival of the species and not around the wellbeing of the individual - adaptive mechanisms of developmental plasticity and phenotypic diversity can produce and conserve forms and functions that are adverse to the individual but that have proved advantageous to the survival of the population and species across a gamut of environmental conditions.

Figure: The FEAR model - the complex adaptive mechanisms determining common psychological conditions

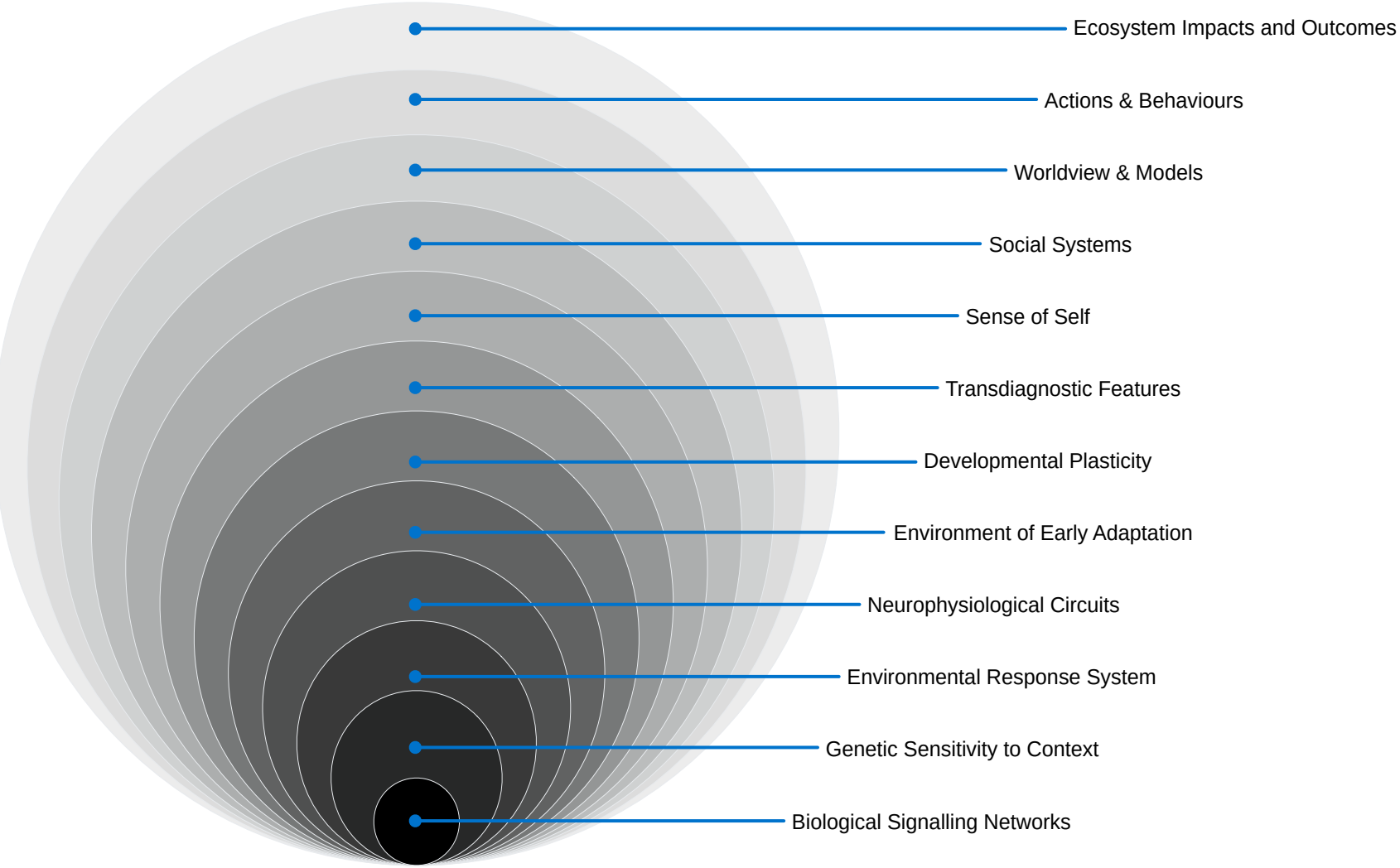


The FEAR model represents a holarchy of nested systems and processes which serve as the principal mechanisms determining the aetiology and course of common psychological conditions. Biological systems are organised in holarchies ${ }^{628}$ - selforganising systems nested within other systems. For example, in the animal body there are microscopic systems such as cellular organelles (eg mitochondria) nested within cells which are nested within tissues which are nested in organs which are nested in functional systems which are nested in networks which are, in turn, nested within and comprise the body as a whole. Holarchies differ from hierarchies in a number of ways - including that the components of a holarchy are simultaneously wholes and parts - self-organising systems interactng with other self-organising systems at all other scales. Further, while hierarchically organised systems tend to be characterised by more or less strict forms of control - meaning specification of form and function of lower levels by higher levels, holarchies tends to be characterised by regulation - meaning greater or lesser degrees of influence exercised between any level and all other levels.

Table: FEAR model systems definitions

\begin{tabular}{|l|l|}
\hline Biological Signalling Networks & $\begin{array}{l}\text { Receptor-Effector systems coupling organisms } \\
\text { with their environment and driving changes in } \\
\text { genetic expression through epigenetic switches }\end{array}$ \\
\hline Genetic Sensitivity to Context & $\begin{array}{l}\text { Representing a shift in biomedical modelling } \\
\text { from genetic vulnerability to 'mental illness' to } \\
\text { sensitivity of genetic expression to both adverse } \\
\text { and advantageous environmental conditions }\end{array}$ \\
\hline Environmental Response System & $\begin{array}{l}\text { Representing a shift from modelling the Nervous } \\
\text { System and particularly the Autonomic Nervous } \\
\text { System as a 'stress response system' to a system } \\
\text { evolved to respond to both adverse and } \\
\text { advantageous conditions }\end{array}$ \\
\hline Neurophysiological Circuits & $\begin{array}{l}\text { Autonomic regulatory control of multiple } \\
\text { systems (visceral, metabolic, cognitive, } \\
\text { affective, endocrine etc) }\end{array}$ \\
\hline Environment of Early Adaptation & $\begin{array}{l}\text { Representing a critical period for determining } \\
\text { developmental plasticity and subsequent life- } \\
\text { course }\end{array}$ \\
\hline Developmental Plasticity & $\begin{array}{l}\text { Adaptive changes in biological structure and } \\
\text { function triggered by environmental interactions } \\
\text { via Biological Signalling Networks }\end{array}$ \\
\hline Transdiagnostic Features & $\begin{array}{l}\text { Common characteristics observed across } \\
\text { diagnostic categories such as activation of } \\
\text { autonomic neurophysiological circuits }\end{array}$ \\
\hline Sense of Self & The biology of Identity \\
\hline Social Systems & Society, community and culture \\
\hline Worldviews and Models & Frames, Beliefs, Theories and Values \\
\hline & \\
\hline
\end{tabular}




\begin{tabular}{|l|l|}
\hline Actions and Behaviours & Patterns of Expression \\
\hline Ecosystem Impacts and Outcomes & Systems Ecology - 'the web of life' \\
\hline
\end{tabular}


1. Henriques-Gomes L. Mental health is Australians' most common ailment, GPs report. The Guardian [Internet]. 2018 Sep 19 [cited 2019 Aug 21]; Available from:

https://www.theguardian.com/australia-news/2018/sep/19/mental-health-is-australians-mostcommon-ailment-gps-report

2. The Royal Australian College of General Practitioners. General Practice: Health of the Nation 2018 [Internet]. East Melbourne, Vic: The Royal Australian College of General Practitioners; 2018 Sep. Available from: https://www.racgp.org.au/general-practice-health-ofthe-nation

3. Wainberg ML, Scorza P, Shultz JM, Helpman L, Mootz JJ, Johnson KA, et al. Challenges and Opportunities in Global Mental Health: a Research-to-Practice Perspective. Curr Psychiatry Rep. 2017 May;19(5):28. doi:10.1007/s11920-017-0780-z

4. Mossialos E, Djordjevic A, R., Osborn, D.Sarnak. International Profiles of Health Care Systems [Internet]. The Commonwealth Fund; 2017 May [cited 2019 Sep 9] p. 182.

Available from: https:/www.commonwealthfund.org/publications/fund-reports/2017/may/ international-profiles-health-care-systems

5. Mendoza J, Elson A, Gilbert Y. Obsessive hope disorder: reflections on 30 years of mental health reform in Australia and visions for the future: Perspectives Report. Caloundra, QLD; 2013.

6. Dobbs R, Manyika J, Woetzel JR. No ordinary disruption: the four global forces breaking all the trends. First edition. New York: PublicAffairs; 2015. 279 p.

7. Farazmand A. Crisis and emergency management: theory and practice. 2014.

8. Frängsmyr T, Heilbron JL, Rider RE, editors. The Quantifying spirit in the 18th century. Berkeley: University of California Press; 1990. 411 p. (Uppsala studies in history of science).

9. Hölzl R. Historicizing Sustainability: German Scientific Forestry in the Eighteenth and Nineteenth Centuries. Science as Culture. 2010 Dec;19(4):431-60.

doi:10.1080/09505431.2010.519866

10. Scott JC. Seeing like a state: how certain schemes to improve the human condition have failed. New Haven: Yale University Press; 1998. 445 p. (Yale agrarian studies).

11. Lang C, Pye O. Blinded by science: The invention of scientific forestry and its influence in the Mekong Region. Watershed. 2000 Nov;6(2):25-34.

12. Oosthoek KJ. The colonial origins of scientific forestry in Britain [Internet]. 2007 [cited 2019 Jan 21]. Available from: https://www.eh-resources.org/colonial-origins-scientific-forestry/

13. Perry DA. The Scientific Basis Of Forestry. Annual Review of Ecology and Systematics. 1998 Nov;29(1):435-66. doi:10.1146/annurev.ecolsys.29.1.435

14. Twain M, De Voto B, Smith HN. Letters from the earth. New York: Harper Perennial; 1991.

15. Held C, Knauff M, Vosgerau G. Mental Models and the Mind: current developments in cognitive psychology, neuroscience, and philosophy of mind [Internet]. Amsterdam; Boston: Elsevier; 2006 [cited 2019 Apr 15]. Available from: http://site.ebrary.com/id/10138469 
16. Rogers K. Scientific modeling [Internet]. Encyclopedia Britannica. 2012 [cited 2019 Jan 22]. Available from: https://www.britannica.com/science/scientific-modeling

17. Loewenthal D, House R. Critically engaging CBT. Maidenhead, Berkshire, England; New York: McGraw Hill Open University Press; 2010.

18. Gentner D, Stevens AL, editors. Mental Models. Routledge; 1983.

19. Simard S. How trees talk to each other [Internet]. [cited 2019 Jan 22]. Available from: https://www.ted.com/talks/suzanne_simard_how_trees_talk_to_each_other

20. de Groot RS, Wilson MA, Boumans RMJ. A typology for the classification, description and valuation of ecosystem functions, goods and services. Ecological Economics. 2002 Jun;41(3):393-408. doi:10.1016/S0921-8009(02)00089-7

21. World Health Organization, Convention on Biological Diversity (Organization), United Nations Environment Programme. Connecting global priorities: biodiversity and human health: a state of knowledge review. [Internet]. 2015 [cited 2019 Feb 20]. Available from: http://apps.who.int/iris/bitstream/10665/174012/1/9789241508537_eng.pdf?ua=1

22. Miller TR. Reconstructing sustainability science: knowledge and action for a sustainable future. London ; New York, NY: Routledge; 2015. 111 p. (Science in society series).

23. Del Giudice M. Evolutionary psychopathology: a unified approach. New York, NY: Oxford University Press; 2018.

24. Sieff DF, editor. Understanding and healing emotional trauma: conversations with pioneering clinicians and researchers. London ; New York, NY: Routledge/ Taylor \& Francis; 2015. 247 p.

25. Gadermann AM, Alonso J, Vilagut G, Zaslavsky AM, Kessler RC. Comorbidity and disease burden in the National Comorbidity Survey Replication (NCS-R). Depress Anxiety. 2012 Sep;29(9):797-806. doi:10.1002/da.21924

26. Moreno-Küstner B, Martín C, Pastor L. Prevalence of psychotic disorders and its association with methodological issues. A systematic review and meta-analyses. McKenna PJ, editor. PLoS ONE. 2018 Apr 12;13(4):e0195687. doi:10.1371/journal.pone.0195687

27. Vigo D, Thornicroft G, Atun R. Estimating the true global burden of mental illness. The Lancet Psychiatry. 2016 Feb;3(2):171-8. doi:10.1016/S2215-0366(15)00505-2

28. Del Giudice M, Ellis BJ, Shirtcliff EA. The Adaptive Calibration Model of stress responsivity. Neuroscience \& Biobehavioral Reviews. 2011 Jun;35(7):1562-92. doi:10.1016/j.neubiorev.2010.11.007

29. trauma | Origin and meaning of trauma by Online Etymology Dictionary. In [cited 2019 Jan 27]. Available from: https://www.etymonline.com/word/trauma

30. Proto-Indo-European language. In: Wikipedia [Internet]. 2019 [cited 2019 Jan 27]. Available from: https://en.wikipedia.org/w/index.php?title=Proto-IndoEuropean_language\&oldid=877979341

31. Trauma | Define Trauma at Dictionary.com. In [cited 2019 Jan 27]. Available from: https://www.dictionary.com/browse/trauma?s=t 
32. Trauma definition and meaning | Collins English Dictionary. In [cited 2019 Jan 27]. Available from: https://www.collinsdictionary.com/dictionary/english/trauma

33. Trauma | Definition of Trauma by Merriam-Webster. In [cited 2019 Jan 27]. Available from: https://www.merriam-webster.com/dictionary/trauma

34. Dorland WAN. Dorland's illustrated medical dictionary. Philadelphia, PA: Elsevier Saunders; 2012.

35. American Psychiatric Association, American Psychiatric Association, editors. Diagnostic and statistical manual of mental disorders: DSM-5. 5th ed. Washington, D.C: American Psychiatric Association; 2013. 947 p.

36. Helms JE, Nicolas G, Green CE. Racism and ethnoviolence as trauma: Enhancing professional training. Traumatology. 2010;16(4):53-62. doi:10.1177/1534765610389595

37. Idsoe T, Dyregrov A, Idsoe EC. Bullying and PTSD Symptoms. Journal of Abnormal Child Psychology. 2012 Aug;40(6):901-11. doi:10.1007/s10802-012-9620-0

38. Nielsen MB, Tangen T, Idsoe T, Matthiesen SB, Magerøy N. Post-traumatic stress disorder as a consequence of bullying at work and at school. A literature review and meta-analysis. Aggression and Violent Behavior. 2015 Mar;21:17-24. doi:10.1016/j.avb.2015.01.001

39. Porges SW. The polyvagal theory: neurophysiological foundations of emotions, attachment, communication, and self-regulation. 1st ed. New York: W. W. Norton; 2011. 347 p. (The Norton series on interpersonal neurobiology).

40. Nakazawa DJ. Childhood disrupted: how your biography becomes your biology, and how you can heal. New York: Atria Books; 2015. 278 p.

41. Jones LK, Cureton JL. Trauma Redefined in the DSM-5: Rationale and Implications for Counseling Practice. The Professional Counselor. 2014 Jul;4(3):257-71. doi:10.15241/lkj.4.3.257

42. ICD-10 Version:2016 [Internet]. [cited 2019 Jan 28]. Available from: https://icd.who.int/browse10/2016/en\#/F43.1

43. VandenBos GR, editor. APA dictionary of psychology (2nd ed.). [Internet]. Washington: American Psychological Association; 2015 [cited 2019 Jan 28]. doi:10.1037/14646-000

44. Caspi A, Moffitt TE. All for One and One for All: Mental Disorders in One Dimension. American Journal of Psychiatry. 2018 Sep;175(9):831-44.

doi:10.1176/appi.ajp.2018.17121383

45. Holman DM, Ports KA, Buchanan ND, Hawkins NA, Merrick MT, Metzler M, et al. The Association Between Adverse Childhood Experiences and Risk of Cancer in Adulthood: A Systematic Review of the Literature. PEDIATRICS. 2016 Nov 1;138(Supplement):S81-91. doi:10.1542/peds.2015-4268L

46. McEwen BS. Protective and Damaging Effects of Stress Mediators. Flier JS, Underhill LH, editors. New England Journal of Medicine. 1998 Jan 15;338(3):171-9.

doi:10.1056/NEJM199801153380307 
47. McEwen BS. Protective and damaging effects of stress mediators: central role of the brain. Dialogues Clin Neurosci. 2006;8(4):367-81.

48. Rethinking the role of stress in development: Emerging evolutionary perspectives | Life Course Research Network (LCRN) [Internet]. 2016 [cited 2019 Jan 28]. (LCRN's series on The State of Life Course Health Development Research - features Marco Del Giudice, $\mathrm{PhD}$.). Available from: http://www.lcrn.net/rethinking-the-role-of-stress-in-developmentemerging-evolutionary-perspectives/

49. World Health Organisation. Depression Fact Sheet [Internet]. 2018 [cited 2019 Jan 28]. Available from: https://www.who.int/en/news-room/fact-sheets/detail/depression

50. Fels A. Time to aim higher. Why mental health must be part of Australia's economic and social reform agenda. [Internet]. National Press Club Address; 2015 Aug 5; National Press Club in Canberra. Available from: http://www.mentalhealthcommission.gov.au/mediacentre/news/national-press-club-address.aspx

51. Victoria Institute of Strategic Economic Studies. The economic cost of serious mental illness and comorbidities in Australia and New Zealand. [Internet]. Royal Australian and New Zealand College of Psychiatrists (RANZCP); 2016. Available from:

https://www.ranzcp.org/files/publications/ranzcp-serious-mental-illness.aspx

52. Roehrig C. Mental Disorders Top The List Of The Most Costly Conditions In The United States: \$201 Billion. Health Affairs. 2016 Jun;35(6):1130-5. doi:10.1377/hlthaff.2015.1659

53. Definition of Etiology [Internet]. Merriam-Webster. [cited 2019 Mar 2]. Available from: https://www.merriam-webster.com/dictionary/etiology

54. Charney DS, editor. Neurobiology of mental illness. 4th ed. New York: Oxford University Press; 2013. 1248 p.

55. Holmes EA, Ghaderi A, Harmer CJ, Ramchandani PG, Cuijpers P, Morrison AP, et al. The Lancet Psychiatry Commission on psychological treatments research in tomorrow's science. The Lancet Psychiatry. 2018 Mar;5(3):237-86. doi:10.1016/S2215-0366(17)30513-8

56. Ioannidis JPA. Research and Theories on the Etiology of Mental Diseases: Doomed to Failure? Psychological Inquiry. 2015 Jul 3;26(3):239-43.

doi:10.1080/1047840X.2015.1021188

57. Deacon BJ. The biomedical model of mental disorder: A critical analysis of its validity, utility, and effects on psychotherapy research. Clinical Psychology Review. 2013 Nov;33(7):846-61. doi:10.1016/j.cpr.2012.09.007

58. Frances A. Saving normal: an insider's revolt against out-of-control psychiatric diagnosis, DSM-5, Big Pharma, and the medicalization of ordinary life. First edition. New York, NY: William Morrow, an imprint of HarperCollins publishers; 2013. 314 p.

59. Kingdon D, Young AH. Research into putative biological mechanisms of mental disorders has been of no value to clinical psychiatry. British Journal of Psychiatry. 2007 Oct;191(04):285-90. doi:10.1192/bjp.bp.107.035675

60. Martins-de-Souza D. Biomarkers for Psychiatric Disorders: Where Are We Standing? Disease Markers. 2013;35:1-2. doi:10.1155/2013/321071 
61. Statement by David Kupfer, MD Chair of DSM-5 Task Force Discusses Future of Mental Health Research [Internet]. American Psychiatric Association; 2013 May. (Release No. 1333). Available from: https://www.madinamerica.com/wp-content/uploads/2013/05/Statementfrom-dsm-chair-david-kupfer-md.pdf

62. Kapur S, Phillips AG, Insel TR. Why has it taken so long for biological psychiatry to develop clinical tests and what to do about it? Molecular Psychiatry. 2012 Dec;17(12):1174-9. doi:10.1038/mp.2012.105

63. Guest PC, Bahn S, editors. Biomarkers of neurological and psychiatric disease. 1. ed. Amsterdam: Elsevier; 2011. 450 p. (International review of neurobiology).

64. Venigalla H, Mekala HM, Hassan M, Ahmed R, Zain H, Dar sabrina, et al. An Update on Biomarkers in Psychiatric Disorders - Are we aware, Do we use in our clinical practice? Mental Health in Family Medicine. 2017;13(3):471-9.

65. Lozupone M, Seripa D, Stella E, La Montagna M, Solfrizzi V, Quaranta N, et al. Innovative biomarkers in psychiatric disorders: a major clinical challenge in psychiatry. Expert Review of Proteomics. 2017 Sep 2;14(9):809-24. doi:10.1080/14789450.2017.1375857

66. Venkatasubramanian G, Keshavan MS. Biomarkers in Psychiatry - A Critique. Annals of Neurosciences. 2016;23(1):3-5. doi:10.1159/000443549

67. Scarr E, Millan MJ, Bahn S, Bertolino A, Turck CW, Kapur S, et al. Biomarkers for Psychiatry: The Journey from Fantasy to Fact, a Report of the 2013 CINP Think Tank: Figure 1. International Journal of Neuropsychopharmacology. 2015 Sep;18(10):pyv042. doi:10.1093/ijnp/pyv042

68. Cohen NL, editor. Public health perspectives on depressive orders. Baltimore: Johns Hopkins University Press; 2017. 442 p.

69. Valderas JM, Starfield B, Sibbald B, Salisbury C, Roland M. Defining Comorbidity: Implications for Understanding Health and Health Services. The Annals of Family Medicine. 2009 Jul 1;7(4):357-63. doi:10.1370/afm.983

70. Atun R. Transitioning health systems for multimorbidity. The Lancet. 2015 Aug;386(9995):721-2. doi:10.1016/S0140-6736(14)62254-6

71. Sartorious N. Comorbidity of mental and physical diseases: a main challenge for medicine of the 21st century. Shanghai Arch Psychiatry. 2013 Apr;25(2):68-9. doi:10.3969/j.issn.10020829.2013.02.002

72. Del Giudice M. An Evolutionary Life History Framework for Psychopathology. Psychological Inquiry. 2014 Oct 2;25(3-4):261-300. doi:10.1080/1047840X.2014.884918

73. Scott KM, Bruffaerts R, Tsang A, Ormel J, Alonso J, Angermeyer MC, et al. Depressionanxiety relationships with chronic physical conditions: Results from the World Mental Health surveys. Journal of Affective Disorders. 2007 Nov;103(1-3):113-20.

doi:10.1016/j.jad.2007.01.015

74. Harvey AG, editor. Cognitive behavioural processes across psychological disorders: a transdiagnostic approach to research and treatment. Oxford ; New York: Oxford University Press; 2004. 365 p. 
75. Kessler RC, Chiu WT, Demler O, Walters EE. Prevalence, Severity, and Comorbidity of 12Month DSM-IV Disorders in the National Comorbidity Survey Replication. Archives of General Psychiatry. 2005 Jun 1;62(6):617. doi:10.1001/archpsyc.62.6.617

76. Plana-Ripoll O, Pedersen CB, Holtz Y, Benros ME, Dalsgaard S, de Jonge P, et al. Exploring Comorbidity Within Mental Disorders Among a Danish National Population. JAMA Psychiatry [Internet]. 2019 Jan 16 [cited 2019 Feb 15]; doi:10.1001/jamapsychiatry.2018.3658

77. Krueger RF, Chentsova-Dutton YE, Markon KE, Goldberg D, Ormel J. A cross-cultural study of the structure of comorbidity among common psychopathological syndromes in the general health care setting. Journal of Abnormal Psychology. 2003;112(3):437-47. doi:10.1037/0021843X.112.3.437

78. Krueger RF. The Structure of Common Mental Disorders. Archives of General Psychiatry. 1999 Oct 1;56(10):921. doi:10.1001/archpsyc.56.10.921

79. Lahey BB, Van Hulle CA, Singh AL, Waldman ID, Rathouz PJ. Higher-Order Genetic and Environmental Structure of Prevalent Forms of Child and Adolescent Psychopathology. Archives of General Psychiatry. 2011 Feb 1;68(2):181. doi:10.1001/archgenpsychiatry.2010.192

80. Krueger RF, Eaton NR. Transdiagnostic factors of mental disorders. World Psychiatry. 2015 Feb;14(1):27-9. doi:10.1002/wps.20175

81. Vollebergh WAM, Iedema J, Bijl RV, de Graaf R, Smit F, Ormel J. The Structure and Stability of Common Mental Disorders: The NEMESIS Study. Archives of General Psychiatry. 2001 Jun 1;58(6):597. doi:10.1001/archpsyc.58.6.597

82. Greenberg G, 3M Company. The book of woe: the DSM and the unmaking of psychiatry [Internet]. New York: Blue Rider Press, a member of Penguin Group (USA) Inc.; 2013 [cited 2019 Jan 29]. Available from:

http://ebookdownload.3m.com/sites/prototypes/web/media/themes/mmm_patron/img/ landing_page/step1.png

83. Nosology | Definition of Nosology by Merriam-Webster. In [cited 2019 Jan 29]. Available from: https://www.merriam-webster.com/dictionary/nosology

84. Aragona M. Epistemological reflections about the crisis of the DSM-5 and the revolutionary potential of the RDoC project. Dial Phil Ment Neuro Sci [Internet]. 2014 Jun;7(1). Available from: http://www.crossingdialogues.com/Ms-A14-08.htm

85. Widiger TA, Samuel DB. Diagnostic categories or dimensions? A question for the Diagnostic and statistical manual of mental disorders--fifth edition. Journal of Abnormal Psychology. 2005;114(4):494-504. doi:10.1037/0021-843X.114.4.494

86. Paris J, Kirmayer LJ. The National Institute of Mental Health Research Domain Criteria: A Bridge Too Far. The Journal of Nervous and Mental Disease. 2016 Jan;204(1):26-32. doi:10.1097/NMD.0000000000000435

87. Viswanath B, Rao NP, Narayanaswamy JC, Sivakumar PT, Kandasamy A, Kesavan M, et al. Discovery biology of neuropsychiatric syndromes (DBNS): a center for integrating clinical 
medicine and basic science. BMC Psychiatry [Internet]. 2018 Dec [cited 2019 Jan 29];18(1). doi:10.1186/s12888-018-1674-2

88. Insel T, Cuthbert B, Garvey M, Heinssen R, Pine DS, Quinn K, et al. Research Domain Criteria (RDoC): Toward a New Classification Framework for Research on Mental Disorders. American Journal of Psychiatry. 2010 Jul;167(7):748-51. doi:10.1176/appi.ajp.2010.09091379

89. van Heugten - van der Kloet D, van Heugten T. The classification of psychiatric disorders according to DSM-5 deserves an internationally standardized psychological test battery on symptom level. Frontiers in Psychology [Internet]. 2015 Aug 4 [cited 2019 Jan 30];6. doi:10.3389/fpsyg.2015.01108

90. Blunt C. Hierarchies of evidence in evidence-based medicine [Internet]. The London School of Economics and Political Science (LSE); 2015. Available from: http://etheses.lse.ac.uk/id/eprint/3284

91. Guyatt GH, Oxman AD, Vist GE, Kunz R, Falck-Ytter Y, Alonso-Coello P, et al. GRADE: an emerging consensus on rating quality of evidence and strength of recommendations. BMJ. 2008 Apr 26;336(7650):924-6. doi:10.1136/bmj.39489.470347.AD

92. Blashfield RK, Keeley JW, Flanagan EH, Miles SR. The Cycle of Classification: DSM-I Through DSM-5. Annual Review of Clinical Psychology. 2014 Mar 28;10(1):25-51. doi:10.1146/annurev-clinpsy-032813-153639

93. Young G. The DSM-5 and the RDoC: Grand Designs and Grander Problems. In: Unifying Causality and Psychology [Internet]. Cham: Springer International Publishing; 2016 [cited 2019 Jan 30]. p. 591-610. doi:10.1007/978-3-319-24094-7_23

94. Melchert TP. Foundations of Professional Psychology: The End of Theoretical Orientations and the Emergence of the Biopsychosocial Approach. First edition. Amsterdam: Elsevier; 2011. 248 p. (Elsevier Insights).

95. Insel TR, Lieberman JA. DSM-5 and RDoC: Shared Interests [Internet]. American Psychiatric Association; 2013. Available from:

https://www.nimh.nih.gov/archive/news/2013/dsm-5-and-rdoc-shared-interests.shtml

96. Kraemer HC. Research Domain Criteria (RDoC) and the DSM - Two Methodological Approaches to Mental Health Diagnosis. JAMA Psychiatry. 2015 Dec 1;72(12):1163. doi:10.1001/jamapsychiatry.2015.2134

97. Sanislow CA. Updating the Research Domain Criteria. World Psychiatry. 2016 Oct;15(3):222-3. doi:10.1002/wps.20374

98. Frank RI, Davidson J. The transdiagnostic road map to case formulation and treatment planning: practical guidance for clinical decision making. Oakland, CA: New Harbinger Publications, Inc; 2014. 239 p.

99. Sauer-Zavala S, Gutner CA, Farchione TJ, Boettcher HT, Bullis JR, Barlow DH. Current Definitions of "Transdiagnostic" in Treatment Development: A Search for Consensus. Behavior Therapy. 2017 Jan;48(1):128-38. doi:10.1016/j.beth.2016.09.004

100. Martin E. Concise medical dictionary. Oxford: Oxford University Press; 2015. 
101. Browse the Medical Dictionary A-Z: Letter T (page 18) | Merriam-Webster [Internet]. [cited 2019 Feb 20]. Available from: https://www.merriam-webster.com/browse/medical/t/18

102. Taber’s Online | Entries T [Internet]. [cited 2019 Feb 20]. Available from: https://www.tabers.com/tabersonline/index/Tabers-Dictionary/Entries/T

103. Dolsen MR, Asarnow LD, Harvey AG. Insomnia as a Transdiagnostic Process in Psychiatric Disorders. Current Psychiatry Reports [Internet]. 2014 Sep [cited 2019 Feb 18];16(9). doi:10.1007/s11920-014-0471-y

104. Capuron L, Castanon N. Role of Inflammation in the Development of Neuropsychiatric Symptom Domains: Evidence and Mechanisms. In: Dantzer R, Capuron L, editors. Inflammation-Associated Depression: Evidence, Mechanisms and Implications [Internet]. Cham: Springer International Publishing; 2016 [cited 2019 Feb 20]. p. 31-44. doi:10.1007/7854_2016_14

105. Rogers GB, Keating DJ, Young RL, Wong M-L, Licinio J, Wesselingh S. From gut dysbiosis to altered brain function and mental illness: mechanisms and pathways. Molecular Psychiatry. 2016 Jun;21(6):738-48. doi:10.1038/mp.2016.50

106. Liu L, Zhu G. Gut-Brain Axis and Mood Disorder. Frontiers in Psychiatry [Internet]. 2018 May 29 [cited 2019 Feb 20];9. doi:10.3389/fpsyt.2018.00223

107. Prescott SL, Millstein RA, Katzman MA, Logan AC. Biodiversity, the Human Microbiome and Mental Health: Moving toward a New Clinical Ecology for the 21st Century? International Journal of Biodiversity. 2016 Aug 3;2016:1-18. doi:10.1155/2016/2718275

108. Skonieczna-Żydecka K, Marlicz W, Misera A, Koulaouzidis A, Łoniewski I. MicrobiomeThe Missing Link in the Gut-Brain Axis: Focus on Its Role in Gastrointestinal and Mental Health. Journal of Clinical Medicine. 2018 Dec 7;7(12):521. doi:10.3390/jcm7120521

109. Goldberger AL. Fractal Variability Versus Pathologic Periodicity: Complexity Loss and Stereotypy in Disease. Perspectives in Biology and Medicine. 1997;40(4):543-61. doi:10.1353/pbm.1997.0063

110. Beauchaine TP, Thayer JF. Heart rate variability as a transdiagnostic biomarker of psychopathology. International Journal of Psychophysiology. 2015 Nov;98(2):338-50. doi:10.1016/j.ijpsycho.2015.08.004

111. Kamath MV, Watanabe M, Upton A. Heart Rate Variability (HRV) Signal Analysis Clinical Applications. 2016.

112. Kemp AH, Quintana DS. The relationship between mental and physical health: Insights from the study of heart rate variability. International Journal of Psychophysiology. 2013 Sep;89(3):288-96. doi:10.1016/j.ijpsycho.2013.06.018

113. De Couck M, Gidron Y. Norms of vagal nerve activity, indexed by Heart Rate Variability, in cancer patients. Cancer Epidemiology. 2013 Oct;37(5):737-41. doi:10.1016/j.canep.2013.04.016

114. Strauss GP, Cohen AS. A Transdiagnostic Review of Negative Symptom Phenomenology and Etiology. Schizophrenia Bulletin. 2017 Jul;43(4):712-9. doi:10.1093/schbul/sbx066 
115. Andrews G, Issakidis C, Sanderson K, Corry J, Lapsley H. Utilising survey data to inform public policy: Comparison of the cost-effectiveness of treatment of ten mental disorders. British Journal of Psychiatry. 2004 Jun;184(06):526-33. doi:10.1192/bjp.184.6.526

116. Brett J, Karanges EA, Daniels B, Buckley NA, Schneider C, Nassir A, et al. Psychotropic medication use in Australia, 2007 to 2015: Changes in annual incidence, prevalence and treatment exposure. Australian \& New Zealand Journal of Psychiatry. 2017 Oct;51(10):9909. doi:10.1177/0004867417721018

117. Mojtabai R, Olfson M. National Trends in Psychotropic Medication Polypharmacy in OfficeBased Psychiatry. Archives of General Psychiatry. 2010 Jan 1;67(1):26. doi:10.1001/archgenpsychiatry.2009.175

118. Whitaker R. Anatomy of an epidemic: magic bullets, psychiatric drugs, and the astonishing rise of mental illness in America. 1st ed. New York: Crown Publishers; 2010. 404 p.

119. World Health Organisation. Schizophrenia WHO Fact Sheet [Internet]. 2018 Apr [cited 2019 Jan 31]. Available from: https://www.who.int/news-room/fact-sheets/detail/schizophrenia

120. Hopper K, editor. Recovery from schizophrenia: an international perspective: a report from the WHO collaborative project, the International Study of Schizophrenia. Oxford ; New York: Oxford University Press; 2007. 370 p.

121. Davey CG, Chanen AM. The unfulfilled promise of the antidepressant medications. The Medical Journal of Australia. 2016 May 16;204(9):348-50. doi:10.5694/mja16.00194

122. Pratt L, Brody D, Gu Q. Antidepressant use among persons aged 12 and over: United States, 2011-2014. NCHS data brief, no 283. [Internet]. Hyattsville, MD: National Center for Health Statistics; 2017 Aug [cited 2019 Jan 31]. Available from: https://www.cdc.gov/nchs/products/databriefs/db283.htm

123. Kirsch I, Deacon BJ, Huedo-Medina TB, Scoboria A, Moore TJ, Johnson BT. Initial Severity and Antidepressant Benefits: A Meta-Analysis of Data Submitted to the Food and Drug Administration. Hay P, editor. PLoS Medicine. 2008 Feb 26;5(2):e45. doi:10.1371/journal.pmed.0050045

124. Cipriani A, Furukawa TA, Salanti G, Chaimani A, Atkinson LZ, Ogawa Y, et al. Comparative efficacy and acceptability of 21 antidepressant drugs for the acute treatment of adults with major depressive disorder: a systematic review and network meta-analysis. The Lancet. 2018 Apr;391(10128):1357-66. doi:10.1016/S0140-6736(17)32802-7

125. Gøtzsche PC. Deadly psychiatry and organised denial. 1. edition. København: People’sPress; 2015. $371 \mathrm{p}$.

126. Boesen K, Paludan-Müller AS, Munkholm K. Network meta-analysis of antidepressants. The Lancet. 2018 Sep;392(10152):1011. doi:10.1016/S0140-6736(18)31783-5

127. Hughes S, Cohen D. A systematic review of long-term studies of drug treated and non-drug treated depression. Journal of Affective Disorders. 2009 Nov;118(1-3):9-18. doi:10.1016/j.jad.2009.01.027

128. Hengartner MP, Angst J, Rössler W. Antidepressant Use Prospectively Relates to a Poorer Long-Term Outcome of Depression: Results from a Prospective Community Cohort Study 
over 30 Years. Psychotherapy and Psychosomatics. 2018;87(3):181-3.

doi:10.1159/000488802

129. Insel TR. The NIMH experimental medicine initiative. World Psychiatry. 2015 Jun;14(2):151-3. doi:10.1002/wps.20227

130. Hengartner MP. Methodological Flaws, Conflicts of Interest, and Scientific Fallacies: Implications for the Evaluation of Antidepressants' Efficacy and Harm. Frontiers in Psychiatry [Internet]. 2017 Dec 7 [cited 2019 Jan 31];8. doi:10.3389/fpsyt.2017.00275

131. Gøtzsche PC, Smith R, Rennie D. Deadly medicines and organised crime: how big pharma has corrupted healthcare. London: Radcliffe Publishing; 2013. 310 p.

132. Lader M. Benzodiazepine harm: how can it be reduced? British Journal of Clinical Pharmacology. 2014 Feb;77(2):295-301. doi:10.1111/j.1365-2125.2012.04418.x

133. R BP. Psychiatric drug-induced Chronic Brain Impairment (CBI): Implications for long-term treatment with psychiatric medication. International Journal of Risk \&amp; Safety in Medicine. 2011;(4):193-200. doi:10.3233/JRS-2011-0542

134. Moncrieff J. The myth of the chemical cure: a critique of psychiatric drug treatment. Basingstoke ; New York: Palgrave Macmillan; 2008. 278 p.

135. Ban TA. Fifty years chlorpromazine: a historical perspective. Neuropsychiatric Disease and Treatment. 2007 Aug;3(4):495-500.

136. U.S. Department of Health and Human Services. Mental Health: A Report of the Surgeon General. [Internet]. Rockville, MD: U.S.: Department of Health and Human Services, Substance Abuse and Mental Health Services Administration, Center for Mental Health Services, National Institutes of Health, National Institute of Mental Health; 1999 p. 487. Available from: https://profiles.nlm.nih.gov/ps/retrieve/ResourceMetadata/NNBBHS

137. France CM, Lysaker PH, Robinson RP. The “chemical imbalance” explanation for depression: Origins, lay endorsement, and clinical implications. Professional Psychology: Research and Practice. 2007;38(4):411-20. doi:10.1037/0735-7028.38.4.411

138. Kemp JJ, Lickel JJ, Deacon BJ. Effects of a chemical imbalance causal explanation on individuals' perceptions of their depressive symptoms. Behaviour Research and Therapy. 2014 May;56:47-52. doi:10.1016/j.brat.2014.02.009

139. Ventola CL. Direct-to-Consumer Pharmaceutical Advertising: Therapeutic or Toxic? P T. 2011 Oct;36(10):669-84.

140. bluesdanny. Zoloft Commercial [Internet]. 2011 [cited 2019 Feb 1]. Available from: https://www.youtube.com/watch?v=51MVDaFh65I

141. Lacasse JR, Leo J. Serotonin and Depression: A Disconnect between the Advertisements and the Scientific Literature. PLoS Medicine. 2005 Nov 8;2(12):e392.

doi:10.1371/journal.pmed.0020392

142. Fernandez Pinto M. To Know or Better Not to. Science \& Technology Studies. 2017 May 11;53. doi:10.23987/sts.61030 
143. Brown \& Williamson Tobacco Corporation - SourceWatch [Internet]. [cited 2019 Feb 1]. Available from: https://www.sourcewatch.org/index.php/Brown_ \%26_Williamson_Tobacco_Corporation

144. Greenhalgh T, Howick J, Maskrey N, for the Evidence Based Medicine Renaissance Group. Evidence based medicine: a movement in crisis? BMJ. 2014 Jun 13;348(jun13 4):g3725g3725. doi:10.1136/bmj.g3725

145. Goldacre B. Bad pharma: how drug companies mislead doctors and harm patients. First American edition. New York: Faber and Faber, Inc., an affiliate of Farrar, Straus and Giroux; 2013. 426 p.

146. Gigerenzer G, Gray JAM, editors. Better doctors, better patients, better decisions: envisioning health care 2020. Cambridge, MA: MIT Press; 2011. 391 p. (Strungmann Forum reports).

147. The NIH is in danger of losing its edge in creating biomedical innovations [Internet]. [cited 2019 Feb 1]. Available from: https://www.statnews.com/2018/01/03/nih-biomedical-researchfunding/

148. Macleod MR, Michie S, Roberts I, Dirnagl U, Chalmers I, Ioannidis JPA, et al. Biomedical research: increasing value, reducing waste. The Lancet. 2014 Jan;383(9912):101-4. doi:10.1016/S0140-6736(13)62329-6

149. Chalmers I, Glasziou P. Avoidable waste in the production and reporting of research evidence. The Lancet. 2009 Jul;374(9683):86-9. doi:10.1016/S0140-6736(09)60329-9

150. Alper BS, Hand JA, Elliott SG, Kinkade S, Hauan MJ, Onion DK, et al. How much effort is needed to keep up with the literature relevant for primary care? J Med Libr Assoc. 2004 Oct;92(4):429-37.

151. McGauran N, Wieseler B, Kreis J, Schüler Y-B, Kölsch H, Kaiser T. Reporting bias in medical research - a narrative review. Trials [Internet]. 2010 Dec [cited 2019 Feb 2];11(1). doi:10.1186/1745-6215-11-37

152. Theisen C, Munaiah N, Al-Zyoud M, Carver JC, Meneely A, Williams L. Attack surface definitions: A systematic literature review. Information and Software Technology. 2018 Dec;104:94-103. doi:10.1016/j.infsof.2018.07.008

153. Spielmans GI, Parry PI. From Evidence-based Medicine to Marketing-based Medicine: Evidence from Internal Industry Documents. Journal of Bioethical Inquiry. 2010 Mar;7(1):13-29. doi:10.1007/s11673-010-9208-8

154. Angell M. The truth about the drug companies: how they deceive us and what to do about it. 1st ed. New York: Random House; 2004. 305 p.

155. Choudhry NK, Stelfox HT, Detsky AS. Relationships between authors of clinical practice guidelines and the pharmaceutical industry. JAMA. 2002 Feb 6;287(5):612-7.

156. Abramson J, Starfield B. The Effect of Conflict of Interest on Biomedical Research and Clinical Practice Guidelines: Can We Trust the Evidence in Evidence-Based Medicine? The Journal of the American Board of Family Medicine. 2005 Sep 1;18(5):414-8. doi:10.3122/jabfm.18.5.414 
157. Shaneyfelt TM, Centor RM. Reassessment of Clinical Practice Guidelines: Go Gently Into That Good Night. JAMA. 2009 Feb 25;301(8):868. doi:10.1001/jama.2009.225

158. Lexchin J. Pharmaceutical industry sponsorship and research outcome and quality: systematic review. BMJ. 2003 May 29;326(7400):1167-70. doi:10.1136/bmj.326.7400.1167

159. Bekelman JE, Li Y, Gross CP. Scope and Impact of Financial Conflicts of Interest in Biomedical Research: A Systematic Review. JAMA. 2003 Jan 22;289(4):454. doi:10.1001/jama.289.4.454

160. Cosgrove L, Krimsky S. A Comparison of DSM-IV and DSM-5 Panel Members' Financial Associations with Industry: A Pernicious Problem Persists. PLoS Medicine. 2012 Mar 13;9(3):e1001190. doi:10.1371/journal.pmed.1001190

161. Meichenbaum D, Lilienfeld SO. How to spot hype in the field of psychotherapy: A 19-item checklist. Professional Psychology: Research and Practice. 2018 Feb;49(1):22-30. doi:10.1037/pro0000172

162. Dattilio FM, Norcross JC. Psychotherapy integration end the emergence of instinctual territoriality. Archives of Psychiatry and Psychotherapy. 2006;8(1):5-16.

163. Wampold BE, Imel ZE. The great psychotherapy debate: the evidence for what makes psychotherapy work. Second edition. New York: Routledge; 2015. 323 p.

164. Wampold BE, Flückiger C, Del Re AC, Yulish NE, Frost ND, Pace BT, et al. In pursuit of truth: A critical examination of meta-analyses of cognitive behavior therapy. Psychotherapy Research. 2017 Jan 2;27(1):14-32. doi:10.1080/10503307.2016.1249433

165. H Kearsley J. Transformative Learning As the Basis for Teaching Healing in the Development of Physician-Healers. Vol. Vol 2. 2015. pp 21.

166. Guilfoyle M. CBT’s integration into societal networks of power. European Journal of Psychotherapy \& Counselling. 2008 Sep;10(3):197-205. doi:10.1080/13642530802337884

167. National Collaborating Centre for mental health. Common mental health disorders: the NICE guideline on identification and pathways to care. Leicester: British Psychological Society; 2011.

168. Hughes T. Cognitive-Behavioural Therapy Evidence Brief [Internet]. New Zealand Government; 2016. Available from:

https://www.justice.govt.nz/assets/Documents/Publications/Cognitive-BehaviouralTherapy.pdf

169. Hughes T. Cognitive-Behavioural Therapy Investment Brief [Internet]. New Zealand Government; 2016. Available from:

https://www.justice.govt.nz/assets/Documents/Publications/investment-brief-CBT.pdf

170. Swann K. A Stepped Care Approach to Mental Health in the Workplace [Internet]. Occupational Health Services Airedale NHS Trust; 2008. Available from: https://www.nice.org.uk/media/default/sharedlearning/251_243supportinginfo.pdf

171. Burkeman O. Therapy wars: the revenge of Freud | Oliver Burkeman. The Guardian [Internet]. 2016 Jan 7 [cited 2019 Jan 25]; Available from: 
https://www.theguardian.com/science/2016/jan/07/therapy-wars-revenge-of-freud-cognitivebehavioural-therapy

172. Swedish National Audit Office. The rehabilitation guarantee is not working - rethink or discontinue [Internet]. 2015 Oct p. 5. Report No.: RIR 2015:19. Available from: https://riksrevisionen.se/en/audit-reports/audit-reports/2015/the-rehabilitation-guarantee-isnot-working---rethink-or-discontinue.html

173. Swedish National Audit Office concludes: When all you have is CBT, mental health suffers [Internet]. [cited 2016 Dec 23]. Available from: http://www.scottdmiller.com/feedbackinformed-treatment-fit/swedish-national-audit-office-concludes-when-all-you-have-is-cbtmental-health-suffers/

174. Revolution in Swedish Mental Health Practice: The Cognitive Behavioral Therapy Monopoly Gives Way [Internet]. [cited 2019 Feb 2]. Available from:

https://www.scottdmiller.com/revolution-in-swedish-mental-health-practice-the-cognitivebehavioral-therapy-monopoly-gives-way/

175. Swedish government moves beyond CBT monopoly, opens door to psychodynamic therapies [Internet]. [cited 2019 Jan 20]. Available from: http://www.philosophyforlife.org/swedenopens-up-cbt-monopoly-gives-nod-to-psychodynamic-therapies/

176. David D, Cristea I, Hofmann SG. Why Cognitive Behavioral Therapy Is the Current Gold Standard of Psychotherapy. Frontiers in Psychiatry [Internet]. 2018 Jan 29 [cited 2019 Jan 23];9. doi:10.3389/fpsyt.2018.00004

177. Leichsenring F, Abbass A, Hilsenroth MJ, Luyten P, Munder T, Rabung S, et al. "Gold Standards," Plurality and Monocultures: The Need for Diversity in Psychotherapy. Frontiers in Psychiatry [Internet]. 2018 Apr 24 [cited 2019 Feb 2];9. doi:10.3389/fpsyt.2018.00159

178. Leichsenring F, Steinert C. Is Cognitive Behavioral Therapy the Gold Standard for Psychotherapy?: The Need for Plurality in Treatment and Research. JAMA. 2017 Oct 10;318(14):1323. doi:10.1001/jama.2017.13737

179. Cuijpers P, Smit F, Bohlmeijer E, Hollon SD, Andersson G. Efficacy of cognitivebehavioural therapy and other psychological treatments for adult depression: meta-analytic study of publication bias. British Journal of Psychiatry. 2010 Mar;196(03):173-8. doi:10.1192/bjp.bp.109.066001

180. Jauhar S, McKenna PJ, Radua J, Fung E, Salvador R, Laws KR. Cognitive-behavioural therapy for the symptoms of schizophrenia: systematic review and meta-analysis with examination of potential bias. British Journal of Psychiatry. 2014 Jan;204(01):20-9. doi:10.1192/bjp.bp.112.116285

181. Jones C, Hacker D, Cormac I, Meaden A, Irving CB. Cognitive behavioural therapy versus other psychosocial treatments for schizophrenia. Cochrane Schizophrenia Group, editor. Cochrane Database of Systematic Reviews [Internet]. 2012 Apr 18 [cited 2019 Feb 2]; doi:10.1002/14651858.CD008712.pub2

182. Leichsenring F, Abbass A, Hilsenroth MJ, Leweke F, Luyten P, Keefe JR, et al. Biases in research: risk factors for non-replicability in psychotherapy and pharmacotherapy research. Psychological Medicine. 2017 Apr;47(06):1000-11. doi:10.1017/S003329171600324X 
183. Hengartner MP. Raising Awareness for the Replication Crisis in Clinical Psychology by Focusing on Inconsistencies in Psychotherapy Research: How Much Can We Rely on Published Findings from Efficacy Trials? Frontiers in Psychology [Internet]. 2018 Feb 28 [cited 2019 Feb 2];9. doi:10.3389/fpsyg.2018.00256

184. Open Science Collaboration. Estimating the reproducibility of psychological science. Science. 2015 Aug 28;349(6251):aac4716-aac4716. doi:10.1126/science.aac4716

185. Ioannidis JPA. Why Most Published Research Findings Are False. PLoS Medicine. 2005;2(8):e124. doi:10.1371/journal.pmed.0020124

186. Price PC, Jhangiani RS, Chiang I-CA, Dana C. Leighton DC, Cuttler C. Research Methods in Psychology [Internet]. 3rd American Edition. Pressbooks; 2017. 272 p. Available from: https://opentext.wsu.edu/carriecuttler/

187. Albagli S, Maciel ML, Abdo AH, Nogueira MCM, Possas SC. Open science, open issues. 2015.

188. Nosek BA. Center for Open Science: Strategic Plan. [cited 2019 Jan 25]; doi:10.31219/osf.io/x2w9h

189. Smith KN, Makel M. Open Science: A Candid Conversation. [cited 2019 Jan 25]; doi:10.31234/osf.io/3869p

190. Kendler KS. What psychiatric genetics has taught us about the nature of psychiatric illness and what is left to learn. Molecular Psychiatry. 2013 Apr 30;18:1058.

191. Phillips J, Frances A, Cerullo MA, Chardavoyne J, Decker HS, First MB, et al. The six most essential questions in psychiatric diagnosis: a pluralogue part 1: conceptual and definitional issues in psychiatric diagnosis. Philosophy, Ethics, and Humanities in Medicine. 2012;7(1):3. doi:10.1186/1747-5341-7-3

192. Kendler KS, Zachar P, Craver C. What kinds of things are psychiatric disorders? Psychological Medicine. 2011 Jun;41(06):1143-50. doi:10.1017/S0033291710001844

193. Clark LA, Cuthbert B, Lewis-Fernández R, Narrow WE, Reed GM. Three Approaches to Understanding and Classifying Mental Disorder: ICD-11, DSM-5, and the National Institute of Mental Health's Research Domain Criteria (RDoC). Psychological Science in the Public Interest. 2017 Nov;18(2):72-145. doi:10.1177/1529100617727266

194. Telles-Correia D, Saraiva S, Gonçalves J. Mental Disorder-The Need for an Accurate Definition. Frontiers in Psychiatry [Internet]. 2018 Mar 12 [cited 2019 Feb 11];9. doi:10.3389/fpsyt.2018.00064

195. Engstrom EJ. Clinical Psychiatry in Imperial Germany: A History of Psychiatric Practice [Internet]. Cornell University Press; 2003. 320 p. (Cornell Studies in the History of Psychiatry). Available from: http://www.jstor.org/stable/10.7591/j.ctv2n7m1k

196. Hyman SE. A glimmer of light for neuropsychiatric disorders. Nature. 2008 Oct;455(7215):890-3. doi:10.1038/nature07454 
197. Hyman SE. New Evidence for Shared Risk Architecture of Mental Disorders. JAMA Psychiatry [Internet]. 2019 Jan 16 [cited 2019 Feb 16];

doi:10.1001/jamapsychiatry.2018.4269

198. Antidepressants don’t work and mental health system is failing, experts say | Stuff.co.nz [Internet]. [cited 2018 Oct 11]. Available from:

https://www.stuff.co.nz/national/health/101759156/antidepressants-dont-work-and-mentalhealth-system-is-failing-experts-say

199. Australian Institute of Health and Welfare. A picture of overweight and obesity in Australia 2017. Canberra: Australian Government. Australian Institute of Health and Welfare; 2017. 60 p. (Cat. no.PHE 216).

200. Taylor MM. The obesity epidemic: why a social justice perspective matters. 2018.

201. Tackling wicked problems: a public policy perspective. Canberra: Australian Public Service Commission; 2007.

202. Page SE. Diversity and complexity. Princeton, NJ: Princeton University Press; 2011. 291 p. (Primers in complex systems).

203. Weaver W. Science and complexity. American Scientist. 1948;36:536.

204. Castellani B. Complexity and the failure of quantitative social science. Discover Society [Internet]. 2014 Nov 4 [cited 2019 Mar 29];(14). Available from: https://discoversociety.org/2014/11/04/focus-complexity-and-the-failure-of-quantitativesocial-science/

205. Hunter DJ, Marks L, Smith KE. The public health system in England. Bristol ; Portland, OR: Policy Press; 2010. 192 p. (Evidence for public health practice series).

206. Szreter S. Rethinking McKeown: the relationship between public health and social change. Am J Public Health. 2002 May;92(5):722-5.

207. Baggott R. Looking Forward to the Past? The Politics of Public Health. Journal of Social Policy. 1991 Apr;20(02):191. doi:10.1017/S0047279400018717

208. Diez Roux AV. Complex Systems Thinking and Current Impasses in Health Disparities Research. Am J Public Health. 2011 Sep;101(9):1627-34. doi:10.2105/AJPH.2011.300149

209. Rutter H, Savona N, Glonti K, Bibby J, Cummins S, Finegood DT, et al. The need for a complex systems model of evidence for public health. The Lancet. 2017 Dec;390(10112):2602-4. doi:10.1016/S0140-6736(17)31267-9

210. Bodeker G, Kronenberg F. A Public Health Agenda for Traditional, Complementary, and Alternative Medicine. Am J Public Health. 2002 Oct;92(10):1582-91. doi:10.2105/AJPH.92.10.1582

211. Hanlon P, Carlisle S, Hannah M, Reilly D, Lyon A. Making the case for a 'fifth wave' in public Health. Public Health. 2011 Jan;125(1):30-6. doi:10.1016/j.puhe.2010.09.004

212. Davies SC, Winpenny E, Ball S, Fowler T, Rubin J, Nolte E. For debate: a new wave in public health improvement. The Lancet. 2014 Nov;384(9957):1889-95. doi:10.1016/S01406736(13)62341-7 
213. Szreter S. Industrialization and health. British Medical Bulletin. 2004 Dec 1;69(1):75-86. doi:10.1093/bmb/ldh005

214. Evans RJ. Epidemics and revolutions: cholera in nineteenth-century Europe. Past and Present. 1988;120(1):123-46. doi:10.1093/past/120.1.123

215. Brown M. Making sense of modernity's maladies: health and disease in the Industrial Revolution. Endeavour. 2006 Sep;30(3):108-12. doi:10.1016/j.endeavour.2006.08.001

216. Fainstein SS. Urban planning - The era of industrialization. In: Encyclopædia Britannica [Internet]. Encyclopædia Britannica, inc.; 2016 [cited 2019 Feb 6]. Available from: https://www.britannica.com/topic/urban-planning/The-era-of-industrialization

217. Abel-Smith B. The Beveridge Report: Its origins and outcomes. International Social Security Review. 1992 Jan;45(1-2):5-16. doi:10.1111/j.1468-246X.1992.tb00900.x

218. The making of modern Britain (1880-1951) - Social Impact of WWII in Britain - Revision 6 - National 5 History - BBC Bitesize [Internet]. [cited 2019 Mar 1]. Available from: https://www.bbc.com/bitesize/guides/z6ctyrd/revision/6

219. Whiteside N. The Beveridge Report and Its Implementation: a Revolutionary Project? Histoire@Politique. 2014;24(3):24. doi:10.3917/hp.024.0024

220. Zweiniger-Bargielowska I. Austerity in Britain: rationing, controls, and consumption, 19391955. Oxford ; New York: Oxford University Press; 2000. 286 p.

221. Timmins N. The five giants: a biography of the welfare state [new edition] [Internet]. London: William Collins; 2017 [cited 2019 Mar 1]. Available from: http://rbdigital.rbdigital.com

222. Milburn A. Slaying the Five Giants: the 75th anniversary of the Beveridge Report. New Statesman America [Internet]. 2017 Nov 29 [cited 2019 Mar 13]; Available from: https://www.newstatesman.com/politics/uk/2017/11/slaying-five-giants-75th-anniversarybeveridge-report

223. Socialism. In: Wikipedia [Internet]. 2019 [cited 2019 Mar 3]. Available from: https://en.wikipedia.org/w/index.php?title=Socialism\&oldid=885859856

224. The Future of the Public's Health in the 21st Century [Internet]. Washington, D.C.: National Academies Press; 2003 [cited 2019 Mar 6]. doi:10.17226/10548

225. Max Roser EO-O, Ritchie H. Life Expectancy. In: Our World in Data [Internet]. 2020. Available from: https://ourworldindata.org/life-expectancy

226. McKinlay JB, McKinlay SM. The Questionable Contribution of Medical Measures to the Decline of Mortality in the United States in the Twentieth Century. The Milbank Memorial Fund Quarterly Health and Society. 1977;55(3):405. doi:10.2307/3349539

227. Lee R. The Demographic Transition: Three Centuries of Fundamental Change. Journal of Economic Perspectives. 2003 Nov;17(4):167-90. doi:10.1257/089533003772034943

228. Shaw M, editor. The widening gap: health inequalities and policy in Britain. Reprinted. Bristol: Policy Press; 2000. 267 p. (Studies in poverty, inequality and social exclusion). 
229. Braveman P, Gottlieb L. The Social Determinants of Health: It's Time to Consider the Causes of the Causes. Public Health Reports. 2014 Jan;129(1_suppl2):19-31.

doi:10.1177/00333549141291S206

230. Bennett JE, Pearson-Stuttard J, Kontis V, Capewell S, Wolfe I, Ezzati M. Contributions of diseases and injuries to widening life expectancy inequalities in England from 2001 to 2016: a population-based analysis of vital registration data. The Lancet Public Health. 2018 Dec;3(12):e586-97. doi:10.1016/S2468-2667(18)30214-7

231. Smith KE, Hill S, Bambra C, editors. Health inequalities: critical perspectives. First edition. Oxford: Oxford University Press; 2016. 314 p.

232. Lyon A. The Fifth Wave. [Internet]. Edinburgh: Scottish Council Foundation; 2003 p. 40. Available from: http://www.iffpraxis.com/u/cms/the_fifth_wave.pdf

233. Susser M, Stein Z. Eras in epidemiology: the evolution of ideas. Oxford ; New York: Oxford University Press; 2009. 352 p.

234. Green LW, Fielding J. The U.S. Healthy People Initiative: Its Genesis and Its Sustainability. Annual Review of Public Health. 2011 Apr 21;32(1):451-70. doi:10.1146/annurevpublhealth-031210-101148

235. Ritchie H, Roser M. Causes of Death. In: Our World in Data [Internet]. 2018 [cited 2019 Mar 19]. Available from: https://ourworldindata.org/causes-of-death

236. WHO Fact Sheet: Cardiovascular diseases (CVDs) [Internet]. World Health Organization; 2017 May [cited 2019 Mar 19]. Available from: https://www.who.int/en/news-room/factsheets/detail/cardiovascular-diseases-(cvds)

237. Causes of Death, Australia, 2017 [Internet]. Australian Bureau of Statistics; 2018 Sep [cited 2019 Mar 19]. Report No.: 3303.0. Available from:

https://www.abs.gov.au/ausstats/abs@.nsf/Lookup/by\%20Subject/3303.0 2017 Main \%20Features Australia's\%20leading\%20causes\%20of\%20death,\%202017 2

238. Weir HK, Anderson RN, Coleman King SM, Soman A, Thompson TD, Hong Y, et al. Heart Disease and Cancer Deaths — Trends and Projections in the United States, 1969-2020.

Preventing Chronic Disease [Internet]. 2016 Nov 17 [cited 2019 Mar 19];13. doi:10.5888/pcd13.160211

239. What do we know about cardiovascular disease spending and outcomes in the United States? - Peterson-Kaiser Health System Tracker [Internet]. [cited 2019 Mar 20]. Available from: https://www.healthsystemtracker.org/chart-collection/know-cardiovascular-disease-spendingoutcomes-united-states/\#item-circulatory-system-diseases-leading-driver-medical-servicesspending-growth-2000-2013

240. Syme SL. Rethinking disease: Where do we go from here? Annals of Epidemiology. 1996 Sep;6(5):463-8. doi:10.1016/S1047-2797(96)00097-X

241. NIH Categorical Spending -NIH Research Portfolio Online Reporting Tools (RePORT) Estimates of Funding for Various Research, Condition, and Disease Categories (RCDC) [Internet]. National Institutes of Health; 2018 May [cited 2019 Mar 19]. Available from: https://report.nih.gov/categorical_spending.aspx 
242. Community Development Interview Series: S. Leonard Syme, University of California, Berkeley - YouTube [Internet]. [cited 2019 Mar 20]. Available from: https://www.youtube.com/watch?v=eU8xTOumoQc

243. Syme SL, Ritterman ML. The Importance of Community Development For Health and WellBeing. Community Development Investment Review, Center for Community Development Investments, Federal Reserve Bank of San Francisco. 2009 Dec;5(3):1-13.

244. Syme SL. The importance of the community as an empowered partner for health and wellbeing [Internet]. Communities in Control Conference; 2009 Jun [cited 2019 Mar 21]; Melbourne, Australia. Available from: http://www.abc.net.au/local/stories/2009/06/15/2598972.htm

245. Ebrahim S, Taylor F, Ward K, Beswick A, Burke M, Davey Smith G. Multiple risk factor interventions for primary prevention of coronary heart disease. Cochrane Heart Group, editor. Cochrane Database of Systematic Reviews [Internet]. 2011 Jan 19 [cited 2019 Mar 21]; doi:10.1002/14651858.CD001561.pub3

246. Multiple risk factor intervention trial. Risk factor changes and mortality results. Multiple Risk Factor Intervention Trial Research Group. JAMA. 1982 Sep 24;248(12):1465-77.

247. Jamner MS, Stokols D, editors. Promoting human wellness: new frontiers for research, practice, and policy. Berkeley: University of California Press; 2000. 737 p.

248. Bezruchka S. Increasing Mortality and Declining Health Status in the USA: Where is Public Health? — Harvard Health Policy Review. Harvard Health Policy Review [Internet]. 2018 Oct 11 [cited 2019 Mar 8]; Available from: http://www.hhpronline.org/articles/2018/10/8/increasing-mortality-and-declining-healthstatus-in-the-usa-where-is-public-health

249. Swan N. Mastering the Control Factor, Part One [Internet]. ABC Radio National; [cited 2019 Mar 25]. Available from:

https://www.abc.net.au/radionational/programs/healthreport/mastering-the-control-factorpart-one/3566112\#transcript

250. McKeown T. The role of medicine: dream, mirage or nemesis? London: Nuffield Provincial Hospitals Trust; 1976. 180 p. (The Rock Carling Fellowship).

251. Graham H. Unequal lives: health and socio-economic inequalities. Maidenhead: Open University Press; 2007. 215 p.

252. Nolte E, McKee M. Does health care save lives?: avoidable mortality revisited. London: Nuffield Trust; 2004. 139 p.

253. Omran AR. The Epidemiologic Transition: A Theory of the Epidemiology of Population Change. Milbank Quarterly. 2005 Dec;83(4):731-57. doi:10.1111/j.1468-0009.2005.00398.x

254. Bunker JP. The role of medical care in contributing to health improvements within societies. Int J Epidemiol. 2001 Dec;30(6):1260-3.

255. McKee M. For debate--Does health care save lives? Croat Med J. 1999 Jun;40(2):123-8. 
256. Næss $\varnothing$. Medical care and population health in the age of health system reforms - the Norwegian experience. Michael Quarterly. 2004;3:236-43.

257. Buck D, Baylis A, Dougall D, Robertson R. Vision For Population Health: towards a healthier future. [Internet]. 11-13 Cavendish Square London W1G 0AN: King's Fund; 2018. 89 p. Available from: www.kingsfund.org.uk

258. Allen J, Goldblatt P, Daly S, Jabbal J, Marmot M. Reducing Health Inequalities Through New Models of Care: A Resource for New Care Models [Internet]. 1-19 Torrington Place, London WC1E 7HB: UCL Institute of Health Equity, Department for Epidemiology \& Public Health, University College London; 2018 Sep p. 45. Available from: www.instituteofhealthequity.org

259. Minkler M. Personal Responsibility for Health? A Review of the Arguments and the Evidence at Century's End. Health Education \& Behavior. 1999 Feb;26(1):121-41. doi:10.1177/109019819902600110

260. Bezruchka S. The Hurrider I Go the Behinder I Get: The Deteriorating International Ranking of U.S. Health Status. Annual Review of Public Health. 2012 Apr 21;33(1):157-73. doi:10.1146/annurev-publhealth-031811-124649

261. Bregman R, Manton E. Utopia for realists: the case for a universal basic income, open borders, and a 15-hour workweek. Amsterdam: De Correspondent; 2016.

262. Haan M, Kaplan GA, Camacho T. Poverty and Health: Prospective Evidence from the Alameda County Study. American Journal of Epidemiology. 1987 Jun;125(6):989-98. doi:10.1093/oxfordjournals.aje.a114637

263. Marmot M. The status syndrome: how your social standing affects our health and longevity. 1. Holt paperbacks ed. New York, NY: Holt; 2005. 319 p.

264. Whitehall II [Internet]. Whitehall II (also known as the Stress and Health Study). [cited 2019 Mar 22]. Available from:

https://www.ucl.ac.uk/iehc/research/epidemiology-public-health/research/whitehallII

265. Institute of Medicine Staff, Practice and Policy, Board on Neuroscience and Behavioral Health. Health and behavior: the interplay of biological, behavioral, and societal influences [Internet]. Washington, D.C.: National Academy Press; 2001 [cited 2019 Mar 25]. Available from: http://site.ebrary.com/id/10032404

266. Rodin J, Schooler C, Schaie KW, editors. Self-directedness: cause and effects throughout the life course. Hillsdale, N.J: L. Erlbaum Associates; 1990. 268 p.

267. Swan N. Mastering the Control Factor, Part Two [Internet]. Health Report. ABC Radio National; 1998 [cited 2019 Mar 25]. Available from:

https://www.abc.net.au/radionational/programs/healthreport/mastering-the-control-factorpart-two/3554360\#transcript

268. Marmot M, Bosma H, Hemingway H, Brunner E, Stansfeld S. Contribution of job control and other risk factors to social variations in coronary heart disease incidence. The Lancet. 1997 Jul;350(9073):235-9. doi:10.1016/S0140-6736(97)04244-X 
269. Berrueta-Clement JR, editor. Changed lives: the effects of the Perry preschool program on youths through age 19. Ypsilanti, Mich: High/Scope Press; 1984. 210 p. (Monographs of the High/Scope Educational Research Foundation).

270. Wren DA, Bedeian AG, Wren DA. The evolution of management thought. 6th ed. Hoboken, N.J. : [Chichester, England: Wiley; Wiley, distributor]; 2009. 532 p.

271. Stewart M. The management myth: debunking modern business philosophy [Internet]. 2009 [cited 2019 Mar 25]. Available from: https://www.overdrive.com/search?q=AD498D6C55A5-459D-8775-9C5B35E3E444

272. Kanki BG, editor. Crew resource management. 2. ed. San Diego, Calif.: Academic Press; 2010. 511 p.

273. Okray R, Lubnau T. Crew resource management for the fire service. Tulsa, Okla: PennWell Corp. : FireEngineering; 2004. 292 p.

274. McChrystal GS. Team of Teams. Penguin Publishing Group; 2015.

275. Neily J, Mills PD, Young-Xu Y, Carney BT, West P, Berger DH, et al. Association Between Implementation of a Medical Team Training Program and Surgical Mortality. JAMA. 2010 Oct 20;304(15):1693. doi:10.1001/jama.2010.1506

276. Schmidt C. Agile software development teams: the impact of agile development on team performance. Mannheim; 2016. 184 p. (Progress in IS).

277. Minkler M, Wallerstein N, editors. Community-based participatory research for health: from process to outcomes. 2nd ed. San Francisco, CA: Jossey-Bass; 2008. 508 p.

278. Wahlquist C. Community-led solutions are key to reducing Aboriginal youth suicide. The Guardian [Internet]. 2019 Feb 7 [cited 2019 Feb 8]; Available from:

https://www.theguardian.com/australia-news/2019/feb/08/community-led-solutions-are-keyto-improving-aboriginal-youth-suicide

279. Lansing JS. Priests and programmers: technologies of power in the engineered landscape of Bali. Princeton, N.J: Princeton University Press; 1991. 183 p.

280. Moritz C, Blackall L, Davis J, Flannery T, Godden L, Head L, et al. Investigation of the causes of mass fish kills in the Menindee Region NSW over the summer of 2018-2019 [Internet]. Australian Academy of Science; 2019 Feb [cited 2019 Apr 13] p. 162. Available from: https://www.science.org.au/files/userfiles/support/reports-and-plans/2019/academyscience-report-mass-fish-kills-digital.pdf

281. Cury P, Mullon C, Garcia S, Shannon L. Viability theory for an ecosystem approach to fisheries. ICES Journal of Marine Science. 2005 May;62(3):577-84. doi:10.1016/j.icesjms.2004.10.007

282. Head BW. Wicked Problems in Public Policy. Public Policy. 2008;3(2):101 - 118.

283. McMillan J. The End Of Banking: Money, Credit, And The Digital Revolution. [Internet]. BookBaby; 2015 [cited 2019 Apr 13]. Available from: http://public.eblib.com/choice/publicfullrecord.aspx?p=2030171 
284. Pettifor A. The production of money: how to break the power of bankers. London: Verso; 2017. $172 \mathrm{p}$.

285. Pupulidy IA. The transformation of accident investigation: from finding cause to sensemaking [PhD Dissertation]. Tilburg University; 2015.

286. Castellani B, Wedgeworth R, Wootton E, Rugle L. A bi-directional theory of addiction: Examining coping and the factors related to substance relapse. Addictive Behaviors. 1997 Jan;22(1):139-44. doi:10.1016/S0306-4603(96)00026-3

287. Homelessness, negative affect, and coping among veterans with gambling problems who misused substances. Psychiatric Services. 1996 Mar;47(3):298-9. doi:10.1176/ps.47.3.298

288. Castellani B. Complexity, cases and health [Internet]. Lecture presented at: 7th ESRC Research Methods Festival; 2016 Jul 5 [cited 2019 Apr 14]; University of Bath. Available from: https://www.youtube.com/watch?v=hN-esSUAGs4\&t=17s

289. Castellani B, Rajaram R, Buckwalter JG, Ball M, Hafferty FW. Place and health as complex systems: a case study and empirical test. 2015.

290. Healthy People - Healthy People 2010 [Internet]. [cited 2019 Apr 16]. Available from: https://www.cdc.gov/nchs/healthy_people/hp2010.htm

291. National Center for Health Statistics (U.S.), editor. Healthy People 2010: final review. Hyattsville, MD: U.S. Dept. of Health and Human Services, Centers for Disease Control and Prevention, National Center for Health Statistics; 2012. 1 p. (DHHS publication).

292. Anderson KM, Institute of Medicine (U.S.), editors. How far have we come in reducing health disparities? Progress since 2000: Workshop summary. Washington, D.C: National Academies Press; 2012. 106 p.

293. Healthy People 2020 Spotlight on Health: Social Determinants of Health [Internet]. Webinar presented at; 2013 Apr 24 [cited 2019 May 6]; Office of Health Promotion and Disease Prevention, U.S. Department of Health and Human Services. Available from: https://www.healthypeople.gov/2020/webinars-events/Webinars-and-Events-Archive

294. Lekkas P, Paquet C, Howard NJ, Daniel M. Illuminating the lifecourse of place in the longitudinal study of neighbourhoods and health. Social Science \& Medicine. 2017 Mar;177:239-47. doi:10.1016/j.socscimed.2016.09.025

295. Anderson S, Bayard M, Bennis P, Cavanagh J, Dolan K, Koshgarian L, et al. The Souls of Poor Folk: Auditing America 50 years after the Poor People's Campaign challenged racism, poverty, the war economy/militarism, and our national morality [Internet]. Washington, DC 20036: Institute for Policy Studies; 2018 Apr p. 123. Available from: https://ips-dc.org/reports/

296. Fink DS, Keyes KM, Cerdá M. Social Determinants of Population Health: A Systems Sciences Approach. Curr Epidemiol Rep. 2016 Mar;3(1):98-105. doi:10.1007/s40471-0160066-8

297. Hankivsky O, Reid C, Cormier R, Varcoe C, Clark N, Benoit C, et al. Exploring the promises of intersectionality for advancing women's health research. Int J Equity Health. 2010;9(1):5. doi:10.1186/1475-9276-9-5 
298. Keppel KG. Ten Largest Racial and Ethnic Health Disparities in the United States based on Healthy People 2010 Objectives. American Journal of Epidemiology. 2007 Apr 9;166(1):97103. doi:10.1093/aje/kwm044

299. Williams DR. Miles to Go before We Sleep: Racial Inequities in Health. J Health Soc Behav. 2012 Sep;53(3):279-95. doi:10.1177/0022146512455804

300. LaVeist TA. Minority populations and health: an introduction to health disparities in the United States. 1st ed. San Francisco: Jossey-Bass; 2005. 348 p.

301. Goodman B, MA. Death By Birth: Bearing the Burden of Maternal Mortality [Internet]. WebMD. [cited 2019 Jun 5]. Available from:

https://www.webmd.com/baby/news/20171116/bearing-burden-of-maternal-mortality

302. How can we reduce black maternal mortality? [Internet]. Mother Jones. [cited 2019 Jun 5]. Available from: https://www.motherjones.com/kevin-drum/2019/05/maternal-mortality/

303. Pregnancy Mortality Surveillance System | Maternal and Infant Health | CDC [Internet]. 2019 [cited 2019 Jun 5]. Available from:

https://www.cdc.gov/reproductivehealth/maternalinfanthealth/pregnancy-mortalitysurveillance-system.htm

304. Cooper RS, Kaufman JS, Ward R. Race and Genomics. N Engl J Med. 2003 Mar 20;348(12):1166-70. doi:10.1056/NEJMsb022863

305. Kuzawa CW, Sweet E. Epigenetics and the embodiment of race: Developmental origins of US racial disparities in cardiovascular health. Am J Hum Biol. 2009 Jan;21(1):2-15. doi:10.1002/ajhb.20822

306. Bryc K, Auton A, Nelson MR, Oksenberg JR, Hauser SL, Williams S, et al. Genome-wide patterns of population structure and admixture in West Africans and African Americans. Proceedings of the National Academy of Sciences. 2010 Jan 12;107(2):786-91. doi:10.1073/pnas.0909559107

307. Williams DR, Priest N, Anderson NB. Understanding associations among race, socioeconomic status, and health: Patterns and prospects. Health Psychology. 2016 Apr;35(4):407-11. doi:10.1037/hea0000242

308. Booth III JN, Li M, Shimbo D, Hess R, Irvin MR, Kittles R, et al. West African Ancestry and Nocturnal Blood Pressure in African Americans: The Jackson Heart Study. American Journal of Hypertension. 2018 May 7;31(6):706-14. doi:10.1093/ajh/hpy038

309. Assari S, Lankarani MM. Race and Urbanity Alter the Protective Effect of Education but not Income on Mortality. Front Public Health [Internet]. 2016 May 20 [cited 2019 May 8];4. doi:10.3389/fpubh.2016.00100

310. Turner RJ, Brown TN, Hale WB. Race, Socioeconomic Position, and Physical Health: A Descriptive Analysis. J Health Soc Behav. 2017 Mar;58(1):23-36.

doi:10.1177/0022146516687008

311. Assari S. Health Disparities due to Diminished Return among Black Americans: Public Policy Solutions: Health Disparities due to Diminished Return among Black Americans. Social Issues and Policy Review. 2018 Jan;12(1):112-45. doi:10.1111/sipr.12042 
312. Assari S, Thomas A, Caldwell CH, Mincy RB. Blacks’ Diminished Health Return of Family Structure and Socioeconomic Status; 15 Years of Follow-up of a National Urban Sample of Youth. J Urban Health. 2018 Feb;95(1):21-35. doi:10.1007/s11524-017-0217-3

313. Nina Martin RM. Nothing Protects Black Women From Dying in Pregnancy and Childbirth [Internet]. ProPublica. 2017 [cited 2019 May 30]. Available from: https://www.propublica.org/article/nothing-protects-black-women-from-dying-in-pregnancyand-childbirth

314. Williams DR, Lawrence JA, Davis BA. Racism and Health: Evidence and Needed Research. Annu Rev Public Health. 2019 Apr;40(1):105-25. doi:10.1146/annurev-publhealth-040218043750

315. Blitstein R. Racism’s Hidden Toll. Pacific Standard [Internet]. 2009 Aug [cited 2019 May 24]; Available from: https://psmag.com/social-justice/racisms-hidden-toll-3643

316. Geronimus AT, Hicken MT, Pearson JA, Seashols SJ, Brown KL, Cruz TD. Do US Black Women Experience Stress-Related Accelerated Biological Aging?: A Novel Theory and First Population-Based Test of Black-White Differences in Telomere Length. Hum Nat. 2010 Mar;21(1):19-38. doi:10.1007/s12110-010-9078-0

317. Kaplan RM, editor. Population health: behavioral and social science insights. Rockville, MD: Agency for Healthcare Research and Quality; 2015. 431 p. (AHRQ publication).

318. Williams DR, Mohammed SA. Racism and Health I: Pathways and Scientific Evidence. American Behavioral Scientist. 2013 Aug;57(8):1152-73. doi:10.1177/0002764213487340

319. Williams DR, Sternthal M. Understanding Racial-ethnic Disparities in Health: Sociological Contributions. J Health Soc Behav. 2010 Mar;51(1_suppl):S15-27.

doi:10.1177/0022146510383838

320. Bailey ZD, Krieger N, Agénor M, Graves J, Linos N, Bassett MT. Structural racism and health inequities in the USA: evidence and interventions. The Lancet. 2017 Apr;389(10077):1453-63. doi:10.1016/S0140-6736(17)30569-X

321. Massey DS, Tannen J. A Research Note on Trends in Black Hypersegregation. Demography. 2015 Jun;52(3):1025-34. doi:10.1007/s13524-015-0381-6

322. Rao S. white people [Internet]. 2019 [cited 2019 Aug 22]. Available from: https://twitter.com/sairasameerarao/status/1163868488630358018

323. Lindley R. The Civil War Through Slaves’ Eyes: An Interview with Andrew Ward [Internet]. [cited 2019 May 12]. Available from: https://historynewsnetwork.org/article/56187

324. Squires GD, editor. From redlining to reinvestment: community responses to urban disinvestment. Philadelphia: Temple University Press; 1992. 288 p. (Conflicts in urban and regional development).

325. Squires GD, editor. Urban sprawl: causes, consequences, \& policy responses. Washington, D.C: Urban Institute Press; 2002. 364 p.

326. Frumkin H, Frank LD, Jackson R. Urban sprawl and public health: designing, planning, and building for healthy communities. Washington, DC: Island Press; 2004. 338 p. 
327. Rothstein R. The color of law: a forgotten history of how our government segregated America. First edition. New York London: Liveright Publishing Corporation, a division of W. W. Norton \& Company; 2017. 345 p.

328. Emanuel N, Collins C, Hoxie J, Asante-Muhammad D. The Road to Zero Wealth: How the Racial Wealth Divide is Hollowing Out America’s Middle Class [Internet]. Washington, DC 20036: The Institute for Policy Studies \& Prosperity Now; 2017 Sep [cited 2019 Apr 19] p. 36. Available from: https://ips-dc.org/report-the-road-to-zero-wealth/

329. Williams DR, Jackson PB. Social Sources Of Racial Disparities In Health. Health Affairs. 2005 Mar;24(2):325-34. doi:10.1377/hlthaff.24.2.325

330. Hummel JR. Emancipating slaves, enslaving free men: a history of the American Civil War [Internet]. 2014 [cited 2019 May 12]. Available from: http://public.eblib.com/choice/publicfullrecord.aspx?p=1486441

331. Feagin JR. Systemic racism: a theory of oppression [Internet]. New York: Routledge; 2006 [cited 2019 Apr 21]. Available from: http://site.ebrary.com/id/10172007

332. Lynching: Targeting Black Veterans [Internet]. Montgomery, Alabama 36104: Equal Justice Initiative; 2017 [cited 2019 Apr 23] p. 52. Available from: https://eji.org/reports/lynching-inamerica-targeting-black-veterans

333. The Color of Law [Internet]. C-SPAN; 2017 [cited 2019 Apr 28]. Available from: https://www.c-span.org/video/?428341-1/the-color-law

334. Nuru-Jeter AM, LaVeist TA. Racial Segregation, Income Inequality, and Mortality in US Metropolitan Areas. J Urban Health. 2011 Apr;88(2):270-82. doi:10.1007/s11524-010-95247

335. Geronimus AT, Pearson JA, Linnenbringer E, Schulz AJ, Reyes AG, Epel ES, et al. RaceEthnicity, Poverty, Urban Stressors, and Telomere Length in a Detroit Community-based Sample. J Health Soc Behav. 2015 Jun;56(2):199-224. doi:10.1177/0022146515582100

336. Moran M. The problem of the past: How historic wrongs became legal problems. University of Toronto Law Journal. 2019 Mar 6;1-52. doi:10.3138/utlj.2018-0063

337. Feagin JR. Racist America: roots, current realities, and future reparations. Third edition. New York: Routledge; 2014. 373 p.

338. Paradies Y, Ben J, Denson N, Elias A, Priest N, Pieterse A, et al. Racism as a Determinant of Health: A Systematic Review and Meta-Analysis. Hills RK, editor. PLoS ONE. 2015 Sep 23;10(9):e0138511. doi:10.1371/journal.pone.0138511

339. Landrine H, Corral I, Lee JGL, Efird JT, Hall MB, Bess JJ. Residential Segregation and Racial Cancer Disparities: A Systematic Review. J Racial and Ethnic Health Disparities. 2017 Dec;4(6):1195-205. doi:10.1007/s40615-016-0326-9

340. Gee GC, Walsemann KM, Brondolo E. A Life Course Perspective on How Racism May Be Related to Health Inequities. Am J Public Health. 2012 May;102(5):967-74. doi:10.2105/AJPH.2012.300666 
341. Wallace S, Nazroo J, Bécares L. Cumulative Effect of Racial Discrimination on the Mental Health of Ethnic Minorities in the United Kingdom. Am J Public Health. 2016 Jul;106(7):1294-300. doi:10.2105/AJPH.2016.303121

342. Cave L, Shepherd C, Cooper M, Zubrick S. Prevalence of Racial Discrimination in a Cohort of Aboriginal and Torres Strait Islander Children [Internet]. , The University of Queensland: Institute for Social Science Researc; 2018 p. 38. (Life Course Centre Working Paper Series, 2018-19). Available from:

https://www.lifecoursecentre.org.au/wp-content/uploads/2018/10/2018-19-LCC-WorkingPaper-Cave-et-al.-1.pdf

343. Shepherd CCJ, Li J, Cooper MN, Hopkins KD, Farrant BM. The impact of racial discrimination on the health of Australian Indigenous children aged 5-10 years: analysis of national longitudinal data. Int J Equity Health. 2017 Dec;16(1):116. doi:10.1186/s12939-0170612-0

344. Hicken MT, Lee H, Ailshire J, Burgard SA, Williams DR. "Every Shut Eye, Ain’t Sleep”: The Role of Racism-Related Vigilance in Racial/Ethnic Disparities in Sleep Difficulty. Race Soc Probl. 2013 Jun;5(2):100-12. doi:10.1007/s12552-013-9095-9

345. Sue DW, Capodilupo CM, Torino GC, Bucceri JM, Holder AMB, Nadal KL, et al. Racial microaggressions in everyday life: Implications for clinical practice. American Psychologist. 2007;62(4):271-86. doi:10.1037/0003-066X.62.4.271

346. Nuru-Jeter A, Dominguez TP, Hammond WP, Leu J, Skaff M, Egerter S, et al. "It's The Skin You're In": African-American Women Talk About Their Experiences of Racism. An Exploratory Study to Develop Measures of Racism for Birth Outcome Studies. Matern Child Health J. 2009 Jan;13(1):29-39. doi:10.1007/s10995-008-0357-x

347. Trinh-Shevrin C, Islam NS, Rey MJ, editors. Asian American communities and health: context, research, policy, and action. 1st ed. San Francisco: Jossey-Bass; 2009. 692 p.

348. Stephens-Davidowitz S. The cost of racial animus on a black candidate: Evidence using Google search data. Journal of Public Economics. 2014 Oct;118:26-40.

doi:10.1016/j.jpubeco.2014.04.010

349. Chae DH, Clouston S, Martz CD, Hatzenbuehler ML, Cooper HLF, Turpin R, et al. Area racism and birth outcomes among Blacks in the United States. Social Science \& Medicine. 2018 Feb;199:49-55. doi:10.1016/j.socscimed.2017.04.019

350. Chae DH, Clouston S, Hatzenbuehler ML, Kramer MR, Cooper HLF, Wilson SM, et al. Association between an Internet-Based Measure of Area Racism and Black Mortality. Zeeb H, editor. PLoS ONE. 2015 Apr 24;10(4):e0122963. doi:10.1371/journal.pone.0122963

351. Making The Case That Discrimination Is Bad For Your Health [Internet]. NPR.org. [cited 2019 May 20]. Available from: https://www.npr.org/sections/codeswitch/2018/01/14/577664626/making-the-case-thatdiscrimination-is-bad-for-your-health

352. Forde AT, Crookes DM, Suglia SF, Demmer RT. The weathering hypothesis as an explanation for racial disparities in health: a systematic review. Annals of Epidemiology. 2019 May;33:118.e3. doi:10.1016/j.annepidem.2019.02.011 
353. Sedgh G, Finer LB, Bankole A, Eilers MA, Singh S. Adolescent Pregnancy, Birth, and Abortion Rates Across Countries: Levels and Recent Trends. Journal of Adolescent Health. 2015 Feb;56(2):223-30. doi:10.1016/j.jadohealth.2014.09.007

354. Larkins SL. Attitudes and behaviours of teenage Indigenous women in Townsville, Australia, with respect to relationships and pregnancy: the "U Mob Yarn Up” Young Parents' Project. [Internet] [PhD thesis]. James Cook University; 2007. Available from: https://researchonline.jcu.edu.au/2036/

355. Wallis C. Children Having Children: Teen Pregnancy in America. Time Magazine. 1985 Dec 9;126(23).

356. Furstenberg FF. Destinies of the disadvantaged: the politics of teenage childbearing. New York: Russell Sage Foundation; 2010.

357. Vinson J. Embodying the problem: the persuasive power of the teenage mother. New Brunswick ; New Jersey: Rutgers University Press; 2018. 237 p.

358. Brodt B, Thornton J, Recktenwald W, Terry D. Cycle Of Poverty, Despair Born Again In Delivery Room. Chicago Tribune [Internet]. 1985 Oct 7 [cited 2019 May 22]; Available from: https://www.chicagotribune.com/news/ct-xpm-1985-10-07-8503080155-story.html

359. Furstenberg F. Teen Pregnancy and Poverty: 30-year-study confirms that living in economically-depressed neighborhoods, not teen motherhood, perpetuates poverty. A briefing paper prepared for the council on contemporary families. [Internet]. Council on Contemporary Families; 2008 Jan [cited 2019 May 20] p. 7. Available from: https://www.contemporaryfamilies.org/wp-content/uploads/2013/10/2008_Briefing_Furstenb erg_Teen-pregnancy-and-poverty.pdf

360. Luker K. Dubious Conceptions: The Controversy Over Teen Pregnancy. The American Prospect [Internet]. 1991 Spring [cited 2019 Jul 13]; Available from: https://prospect.org/article/dubious-conceptions-controversy-over-teen-pregnancy

361. Geronimus A, Korenman S. The Socioeconomic Consequences of Teen Childbearing Reconsidered [Internet]. Cambridge, MA: National Bureau of Economic Research; 1991 May [cited 2019 May 22] p. w3701. Report No.: w3701. doi:10.3386/w3701

362. Furstenberg F. Reconsidering Teenage Pregnancy and Parenthood. Societies. 2016 Nov 1;6(4):33. doi:10.3390/soc6040033

363. Hoffman SD. Teenage Childbearing Is Not So Bad After All...Or Is It? A Review of the New Literature. Family Planning Perspectives. 1998 Sep;30(5):236. doi:10.2307/2991610

364. Geronimus AT, Thompson JP. To Denigrate, Ignore, Or Disrupt: Racial Inequality in Health and the Impact of a Policy-induced Breakdown of African American Communities. Du Bois Review: Social Science Research on Race [Internet]. 2004 Sep [cited 2019 May 24];1(02). doi:10.1017/S1742058X04042031

365. Bradbury B. The impact of young motherhood on education, employment and marriage [Internet]. Sydney, NSW: Social Policy Research Centre, Univ. of New South Wales; 2006 [cited 2019 May 30]. Available from: http://www.sprc.unsw.edu.au/dp/DP148.pdf 
366. Rozman A. Life History Theory and School-Age Pregnancy: Review and Application [Internet] [Honors Scholar Theses]. University of Connecticut; 2015. Available from: http://digitalcommons.uconn.edu/srhonors_theses/425

367. Snapshot [Internet]. [cited 2019 May 30]. Available from: https://www.who.int/news-room/fact-sheets/detail/adolescent-pregnancy

368. Chen X-K, Wen SW, Fleming N, Demissie K, Rhoads GG, Walker M. Teenage pregnancy and adverse birth outcomes: a large population based retrospective cohort study. International Journal of Epidemiology. 2007 Apr 1;36(2):368-73. doi:10.1093/ije/dyl284

369. LeGrand TK, Mbacke CSM. Teenage Pregnancy and Child Health in the Urban Sahel. Studies in Family Planning [Internet]. 1993 May [cited 2019 May 30];24(3). doi:10.2307/2939229

370. Taffa N, Obare F. Pregnancy and child health outcomes among adolescents in Ethiopia. EJHD [Internet]. 2004;18(2). Available from: https://ejhd.org/index.php/ejhd/article/view/680

371. Kawakita T, Wilson K, Grantz KL, Landy HJ, Huang C-C, Gomez-Lobo V. Adverse Maternal and Neonatal Outcomes in Adolescent Pregnancy. Journal of Pediatric and Adolescent Gynecology. 2016 Apr;29(2):130-6. doi:10.1016/j.jpag.2015.08.006

372. Gibbs CM, Wendt A, Peters S, Hogue CJ. The Impact of Early Age at First Childbirth on Maternal and Infant Health. Paediatric and Perinatal Epidemiology. 2012 Jul;26:259-84. doi:10.1111/j.1365-3016.2012.01290.x

373. Lassi ZS, Majeed A, Rashid S, Yakoob MY, Bhutta ZA. The interconnections between maternal and newborn health - evidence and implications for policy. The Journal of Maternal-Fetal \& Neonatal Medicine. 2013 May;26(sup1):3-53. doi:10.3109/14767058.2013.784737

374. Arline Geronimus: Q\&A About Weathering, or How Chronic Stress Prematurely Ages Your Body [Internet]. EverydayHealth.com. [cited 2019 May 23]. Available from: https://www.everydayhealth.com/wellness/united-states-of-stress/advisory-board/arline-tgeronimus-q-a/

375. Gallo LC, Fortmann AL, Mattei J. Allostatic Load and the Assessment of Cumulative Biological Risk in Biobehavioral Medicine: Challenges and Opportunities. Psychosomatic Medicine. 2014 Sep;76(7):478-80. doi:10.1097/PSY.0000000000000095

376. Geronimus AT, Hicken M, Keene D, Bound J. "Weathering” and Age Patterns of Allostatic Load Scores Among Blacks and Whites in the United States. Am J Public Health. 2006 May;96(5):826-33. doi:10.2105/AJPH.2004.060749

377. Shalev I. Early life stress and telomere length: Investigating the connection and possible mechanisms: A critical survey of the evidence base, research methodology and basic biology. Bioessays. 2012 Nov;34(11):943-52. doi:10.1002/bies.201200084

378. Drury SS, Theall K, Gleason MM, Smyke AT, De Vivo I, Wong JYY, et al. Telomere length and early severe social deprivation: linking early adversity and cellular aging. Mol Psychiatry. 2012 Jul;17(7):719-27. doi:10.1038/mp.2011.53 
379. Jackson SP, Bartek J. The DNA-damage response in human biology and disease. Nature. 2009 Oct;461(7267):1071-8. doi:10.1038/nature08467

380. Adams JA, Galloway TS, Mondal D, Esteves SC, Mathews F. Effect of mobile telephones on sperm quality: A systematic review and meta-analysis. Environment International. 2014 Sep;70:106-12. doi:10.1016/j.envint.2014.04.015

381. Shay JW. Telomerase and cancer. Human Molecular Genetics. 2001 Apr 1;10(7):677-85. doi:10.1093/hmg/10.7.677

382. Blaze J, Asok A, Roth TL. The long-term impact of adverse caregiving environments on epigenetic modifications and telomeres. Front Behav Neurosci [Internet]. 2015 Apr 8 [cited 2019 Jun 17];9. doi:10.3389/fnbeh.2015.00079

383. Shalev I, Moffitt TE, Sugden K, Williams B, Houts RM, Danese A, et al. Exposure to violence during childhood is associated with telomere erosion from 5 to 10 years of age: a longitudinal study. Mol Psychiatry. 2013 May;18(5):576-81. doi:10.1038/mp.2012.32

384. Okuda K, Bardeguez A, Gardner JP, Rodriguez P, Ganesh V, Kimura M, et al. Telomere Length in the Newborn. Pediatric Research. 2002 Sep 1;52(3):377-81.

doi:10.1203/00006450-200209000-00012

385. Drury SS, Esteves K, Hatch V, Woodbury M, Borne S, Adamski A, et al. Setting the Trajectory: Racial Disparities in Newborn Telomere Length. The Journal of Pediatrics. 2015 May;166(5):1181-6. doi:10.1016/j.jpeds.2015.01.003

386. Rewak M, Buka S, Prescott J, De Vivo I, Loucks EB, Kawachi I, et al. Race-related health disparities and biological aging: Does rate of telomere shortening differ across blacks and whites? Biological Psychology. 2014 May;99:92-9. doi:10.1016/j.biopsycho.2014.03.007

387. Chae DH, Nuru-Jeter AM, Adler NE, Brody GH, Lin J, Blackburn EH, et al. Discrimination, Racial Bias, and Telomere Length in African-American Men. American Journal of Preventive Medicine. 2014 Feb;46(2):103-11. doi:10.1016/j.amepre.2013.10.020

388. Stearns SC. Trade-Offs in Life-History Evolution. Functional Ecology. 1989;3(3):259. doi:10.2307/2389364

389. Salguero-Gómez R, Jones OR, Jongejans E, Blomberg SP, Hodgson DJ, Mbeau-Ache C, et al. Fast-slow continuum and reproductive strategies structure plant life-history variation worldwide. Proc Natl Acad Sci USA. 2016 Jan 5;113(1):230-5. doi:10.1073/pnas.1506215112

390. Zera AJ, Harshman LG. The Physiology of Life History Trade-Offs in Animals. Annu Rev Ecol Syst. 2001 Nov;32(1):95-126. doi:10.1146/annurev.ecolsys.32.081501.114006

391. Keen EC. Tradeoffs in bacteriophage life histories. Bacteriophage. 2014 Apr;4(2):e28365. doi:10.4161/bact.28365

392. West-Eberhard MJ. Developmental plasticity and evolution. Oxford ; New York: Oxford University Press; 2003. 794 p. 
393. Benard MF. Predator-Induced Phenotypic Plasticity in Organisms with Complex Life Histories. Annu Rev Ecol Evol Syst. 2004 Dec 15;35(1):651-73.

doi:10.1146/annurev.ecolsys.35.021004.112426

394. DeWitt TJ, Scheiner SM, editors. Phenotypic plasticity: functional and conceptual approaches. Oxford ; New York: Oxford University Press; 2004. 247 p.

395. Ebert D. Ecology, epidemiology, and evolution of parasitism in Daphnia. Bethesda, MD: National Center for Biotechnology Information; 2005.

396. Weiss LC. Sensory Ecology of Predator-Induced Phenotypic Plasticity. Front Behav Neurosci. 2019 Jan 18;12:330. doi:10.3389/fnbeh.2018.00330

397. Engel K, Tollrian R. Inducible defences as key adaptations for the successful invasion of Daphnia lumholtzi in North America? Proceedings of the Royal Society B: Biological Sciences. 2009 May 22;276(1663):1865-73. doi:10.1098/rspb.2008.1861

398. Ellis BJ, Del Giudice M. Developmental Adaptation to Stress: An Evolutionary Perspective. Annu Rev Psychol. 2019 Jan 4;70(1):111-39. doi:10.1146/annurev-psych-122216-011732

399. Bateson P, Gluckman P, Hanson M. The biology of developmental plasticity and the Predictive Adaptive Response hypothesis: Developmental plasticity and the PAR response. J Physiol. 2014 Jun 1;592(11):2357-68. doi:10.1113/jphysiol.2014.271460

400. Sultan SE. Developmental plasticity: re-conceiving the genotype. Interface Focus. 2017 Oct 6;7(5):20170009. doi:10.1098/rsfs.2017.0009

401. Tollrian R. Predator-Induced Morphological Defenses: Costs, Life History Shifts, and Maternal Effects in Daphnia Pulex. Ecology. 1995 Sep;76(6):1691-705. doi:10.2307/1940703

402. Griskevicius V, Delton AW, Robertson TE, Tybur JM. Environmental contingency in life history strategies: The influence of mortality and socioeconomic status on reproductive timing. Journal of Personality and Social Psychology. 2011;100(2):241-54. doi:10.1037/a0021082

403. Geary DC. Sexual Selection and Human Life History. In: Advances in Child Development and Behavior [Internet]. Elsevier; 2003 [cited 2019 Jun 5]. p. 41-101. doi:10.1016/S00652407(02)80039-8

404. Lea AJ, Tung J, Archie EA, Alberts SC. Developmental plasticity: Bridging research in evolution and human health. Evolution, Medicine, and Public Health. 2017 Jan 1;2017(1):162-75. doi:10.1093/emph/eox019

405. Mumby HS, Mar KU, Hayward AD, Htut W, Htut-Aung Y, Lummaa V. Elephants born in the high stress season have faster reproductive ageing. Scientific Reports. 2015 Sep 14;5:13946.

406. Vitzthum VJ. The ecology and evolutionary endocrinology of reproduction in the human female. Am J Phys Anthropol. 2009;140(S49):95-136. doi:10.1002/ajpa.21195

407. Volk AA, Atkinson JA. Infant and child death in the human environment of evolutionary adaptation. Evolution and Human Behavior. 2013 May;34(3):182-92.

doi:10.1016/j.evolhumbehav.2012.11.007 
408. Kramer KL, Lancaster JB. Teen motherhood in cross-cultural perspective. Annals of Human Biology. 2010 Oct;37(5):613-28. doi:10.3109/03014460903563434

409. Ellis BJ, Figueredo AJ, Brumbach BH, Schlomer GL. Fundamental Dimensions of Environmental Risk: The Impact of Harsh versus Unpredictable Environments on the Evolution and Development of Life History Strategies. Hum Nat. 2009 Jun;20(2):204-68. doi:10.1007/s12110-009-9063-7

410. Nettle D. Dying young and living fast: variation in life history across English neighborhoods. Behavioral Ecology. 2010;21(2):387-95. doi:10.1093/beheco/arp202

411. Mell H, Safra L, Algan Y, Baumard N, Chevallier C. Childhood environmental harshness predicts coordinated health and reproductive strategies: A cross-sectional study of a nationally representative sample from France. Evolution and Human Behavior. 2018 Jan;39(1):1-8. doi:10.1016/j.evolhumbehav.2017.08.006

412. Clutterbuck S, Adams J, Nettle D. Frequent Residential Relocations Cumulatively Accelerate Menarcheal Timing In A Sample Of English Adolescent Girls. J Biosoc Sci. 2015 Mar;47(2):188-202. doi:10.1017/S0021932014000157

413. Poiani A, editor. Pragmatic evolution: applications of evolutionary theory. Cambridge ; New York: Cambridge University Press; 2012. 340 p.

414. Furstenberg FF. As the Pendulum Swings: Teenage Childbearing and Social Concern. Family Relations. 1991 Apr;40(2):127. doi:10.2307/585470

415. Johns SE, Dickins TE, Clegg HT. Teenage pregnancy and motherhood: How might evolutionary theory inform policy? Journal of Evolutionary Psychology. 2011 Mar;9(1):3-19. doi:10.1556/JEP.9.2011.37.1

416. McCall SJ, Bhattacharya S, Okpo E, Macfarlane GJ. Evaluating the social determinants of teenage pregnancy: a temporal analysis using a UK obstetric database from 1950 to 2010. J Epidemiol Community Health. 2015 Jan;69(1):49-54. doi:10.1136/jech-2014-204214

417. Smith K, Colman S. Evaluation of Adolescent Pregnancy Prevention Approaches: Design of the Impact Study [Internet]. Mathematica Policy Research; undated. Report No.: 159b7fa95d6c4f58b4b1eefbc. Available from: https://ideas.repec.org/p/mpr/mprres/159b7fa95d6c4f58b4b1eefbca266249.html

418. Chandra-Mouli V, Lane C, Wong S. What Does Not Work in Adolescent Sexual and Reproductive Health: A Review of Evidence on Interventions Commonly Accepted as Best Practices. Global Health: Science and Practice. 2015 Sep 10;3(3):333. doi:10.9745/GHSP-D15-00126

419. Aslam RW, Hendry M, Booth A, Carter B, Charles JM, Craine N, et al. Intervention Now to Eliminate Repeat Unintended Pregnancy in Teenagers (INTERUPT): a systematic review of intervention effectiveness and cost-effectiveness, and qualitative and realist synthesis of implementation factors and user engagement. BMC Med. 2017 Dec;15(1):155. doi:10.1186/s12916-017-0904-7

420. Lindberg L, Boonstra H. Despite new branding, abstinence-only programs don't work. The Hill [Internet]. 201712 [cited 2019 Jul 15]; Available from: 
https://thehill.com/opinion/healthcare/363380-despite-new-branding-abstinence-onlyprograms-dont-work

421. Crespi EJ, Denver RJ. Ancient origins of human developmental plasticity. Am J Hum Biol. 2005 Jan;17(1):44-54. doi:10.1002/ajhb.20098

422. Dobzhansky T. Biology, Molecular And Organismic. Am Zool. 1964 Nov;4(4):443-52. doi:10.1093/icb/4.4.443

423. Millstein RL. Evolution. In: Zalta EN, editor. The Stanford Encyclopedia of Philosophy [Internet]. Summer 2019. Metaphysics Research Lab, Stanford University; 2019. Available from: https://plato.stanford.edu/archives/sum2019/entries/evolution/

424. Pigliucci M, Müller G, Konrad Lorenz Institute for Evolution and Cognition Research, editors. Evolution, the extended synthesis. Cambridge, Mass: MIT Press; 2010. 495 p.

425. Modern synthesis (20th century). In: Wikipedia [Internet]. 2019 [cited 2019 Aug 6]. Available from: https://en.wikipedia.org/w/index.php?

title=Modern_synthesis_(20th_century)\&oldid=909534357

426. El Karkouri K, Kowalczewska M, Armstrong N, Azza S, Fournier P-E, Raoult D. Multiomics Analysis Sheds Light on the Evolution and the Intracellular Lifestyle Strategies of Spotted Fever Group Rickettsia spp. Front Microbiol. 2017 Jul 20;8:1363. doi:10.3389/fmicb.2017.01363

427. Smith CM, Ruppell JC. What Anthropologists Should Know About the New Evolutionary Synthesis. Structure and Dynamics [Internet]. 2011;5(2). Available from: https://escholarship.org/uc/item/18b9f0jb

428. Koonin EV. Towards a postmodern synthesis of evolutionary biology. Cell Cycle. 2009 Mar 15;8(6):799-800. doi:10.4161/cc.8.6.8187

429. Silverman PH. Rethinking genetic determinism: with only 30,000 genes, what is it that makes humans human? The Scientist. 2004 May;24:32.

430. Queller DC, Strassmann JE. Problems of multi-species organisms: endosymbionts to holobionts. Biol Philos. 2016 Nov;31(6):855-73. doi:10.1007/s10539-016-9547-x

431. Feero WG, Guttmacher AE, Collins FS. Genomic Medicine - An Updated Primer. Feero WG, Guttmacher AE, editors. N Engl J Med. 2010 May 27;362(21):2001-11. doi:10.1056/NEJMra0907175

432. The Editors of Encyclopaedia Britannica. Gene. In: Encyclopedia Britannica [Internet]. Encyclopædia Britannica, inc.; 2019 [cited 2019 Aug 12]. Available from: https://www.britannica.com/science/gene

433. Noble D. The music of life: biology beyond the genome. Oxford ; New York: Oxford University Press; 2006. 153 p.

434. International Human Genome Sequencing Consortium- Describes Finished Human Genome Sequence | NHGRI [Internet]. Bethesda, MD: national Human Genome Research Institute; 2004 Oct [cited 2019 Aug 13]. (NIH News Release). Available from: https://www.genome.gov/12513430/2004-release-ihgsc-describes-finished-human-sequence 
435. Anderson S, Bankier AT, Barrell BG, de Bruijn MHL, Coulson AR, Drouin J, et al. Sequence and organization of the human mitochondrial genome. Nature. 1981 Apr;290(5806):457-65. doi:10.1038/290457a0

436. Altman LK. Reading The Book Of Life: The Doctor's World; Genomic Chief Has High Hopes, and Great Fears, for Genetic Testing. The New York Times [Internet]. 2000 Jun 27 [cited 2019 Aug 13]; Available from: https://www.nytimes.com/2000/06/27/science/readingbook-life-doctor-s-world-genomic-chief-has-high-hopes-great-fears-for.html

437. Lander ES, Linton LM, Birren B, Nusbaum C, Zody MC, Baldwin J, et al. Initial sequencing and analysis of the human genome. Nature. 2001 Feb 1;409(6822):860-921. doi:10.1038/35057062

438. Srivastava M, Simakov O, Chapman J, Fahey B, Gauthier MEA, Mitros T, et al. The Amphimedon queenslandica genome and the evolution of animal complexity. Nature. 2010 Aug 5;466:720.

439. Hodgkin J. What does a worm want with 20,000 genes? Genome Biol. 2001;2(11):COMMENT2008. doi:10.1186/gb-2001-2-11-comment2008

440. Eddy SR. The C-value paradox, junk DNA and ENCODE. Current Biology. 2012 Nov;22(21):R898-9. doi:10.1016/j.cub.2012.10.002

441. Doolittle WF. Is junk DNA bunk? A critique of ENCODE. Proceedings of the National Academy of Sciences. 2013 Apr 2;110(14):5294-300. doi:10.1073/pnas.1221376110

442. Fedorov A, Fedorova L. Where is the difference between the genomes of humans and annelids? Genome Biol. 2006;7(1):203. doi:10.1186/gb-2006-7-1-203

443. Porta M. The genome sequence is a jazz score. International Journal of Epidemiology. 2003 Feb;32(1):29-31. doi:10.1093/ije/dyg015

444. Dobbs D, Dupré J, James K, Hercher L, Sapolsky R. Dead or alive? An expert roundtable on the Selfish Gene | Aeon Essays. Aeon [Internet]. [cited 2019 Aug 14]; Available from: https://aeon.co/essays/dead-or-alive-an-expert-roundtable-on-the-selfish-gene

445. Dobbs D. Die, selfish gene, die. Aeon [Internet]. 2013 Dec 3 [cited 2019 Aug 14]; Available from: https://aeon.co/essays/the-selfish-gene-is-a-great-meme-too-bad-it-s-so-wrong

446. Noble D. Dance to the tune of life: biological relativity. Cambridge ; New York: Cambridge University Press; 2017. 283 p.

447. Munoz-Lopez M, Garcia-Perez J. DNA Transposons: Nature and Applications in Genomics. CG. 2010 Apr 1;11(2):115-28. doi:10.2174/138920210790886871

448. Bonnivard E, Higuet D. Fluidity of eukaryotic genomes. Comptes Rendus Biologies. 2009 Feb;332(2-3):234-40. doi:10.1016/j.crvi.2008.09.005

449. Greenwood V. One Gene, Many Proteins. Quanta Magazine [Internet]. 2016 Apr 26 [cited 2019 Aug 10]; Available from: https://www.quantamagazine.org/one-gene-many-proteins20160426/

450. Ayala FJ. The Mechanisms of Evolution. Sci Am. 1978 Sep;239(3):56-69.

doi:10.1038/scientificamerican0978-56 
451. Medini D, Donati C, Tettelin H, Masignani V, Rappuoli R. The microbial pan-genome. Current Opinion in Genetics \& Development. 2005 Dec;15(6):589-94.

doi:10.1016/j.gde.2005.09.006

452. Koonin EV. The logic of chance: the nature and origin of biological evolution. Upper Saddle River, N.J: Pearson Education; 2012. 516 p.

453. Koonin EV. The Origin at 150: is a new evolutionary synthesis in sight? Trends in Genetics. 2009 Nov;25(11):473-5. doi:10.1016/j.tig.2009.09.007

454. Chen D-S, Wu Y-Q, Zhang W, Jiang S-J, Chen S-Z. Horizontal gene transfer events reshape the global landscape of arm race between viruses and homo sapiens. Sci Rep. 2016 Jun;6(1):26934. doi:10.1038/srep26934

455. Crisp A, Boschetti C, Perry M, Tunnacliffe A, Micklem G. Expression of multiple horizontally acquired genes is a hallmark of both vertebrate and invertebrate genomes. Genome Biology. 2015 Mar 13;16(1):50. doi:10.1186/s13059-015-0607-3

456. Rose MR, Oakley TH. The new biology: beyond the Modern Synthesis. Biol Direct. 2007;2(1):30. doi:10.1186/1745-6150-2-30

457. Bohacek J, Mansuy IM. Molecular insights into transgenerational non-genetic inheritance of acquired behaviours. Nat Rev Genet. 2015 Nov;16(11):641-52. doi:10.1038/nrg3964

458. voicesfromoxfordUK. Prof Denis Noble - Dance to the Tune of Life Lecture [Internet]. 2016 [cited 2019 Aug 20]. Available from: https://www.youtube.com/watch?v=j3WenGjyokg

459. Orgogozo V, editor. Genes and evolution. First edition. Amsterdam ; Boston: Elsevier/AP, Academic Press is an imprint of Elsevier; 2016. 401 p. (Current topics in developmental biology).

460. Lappin TRJ, Grier DG, Thompson A, Halliday HL. HOX genes: seductive science, mysterious mechanisms. Ulster Med J. 2006 Jan;75(1):23-31.

461. Biémont C, Vieira C. Junk DNA as an evolutionary force. Nature. 2006 Oct 1;443(7111):521-4. doi:10.1038/443521a

462. Champagne FA. Epigenetic mechanisms and the transgenerational effects of maternal care. Frontiers in Neuroendocrinology. 2008 Jun;29(3):386-97. doi:10.1016/j.yfrne.2008.03.003

463. Shapiro JA. Evolution: a view from the 21st century. Upper Saddle River, N.J: FT Press Science; 2011. 253 p.

464. Odegard VH, Schatz DG. Targeting of somatic hypermutation. Nat Rev Immunol. 2006 Aug;6(8):573-83. doi:10.1038/nri1896

465. Kohl P, Crampin EJ, Quinn TA, Noble D. Systems Biology: An Approach. Clin Pharmacol Ther. 2010 Jul;88(1):25-33. doi:10.1038/clpt.2010.92

466. Barabási A-L, Oltvai ZN. Network biology: understanding the cell’s functional organization. Nat Rev Genet. 2004 Feb;5(2):101-13. doi:10.1038/nrg1272 
467. Titz B, Rajagopala SV, Goll J, Häuser R, McKevitt MT, Palzkill T, et al. The Binary Protein Interactome of Treponema pallidum - The Syphilis Spirochete. Hall N, editor. PLoS ONE. 2008 May 28;3(5):e2292. doi:10.1371/journal.pone.0002292

468. Enberg K, Jørgensen C, Dunlop ES, Varpe Ø, Boukal DS, Baulier L, et al. Fishing-induced evolution of growth: concepts, mechanisms and the empirical evidence. Marine Ecology. 2012 Mar;33(1):1-25. doi:10.1111/j.1439-0485.2011.00460.x

469. Svdmolen. Precis octavia, Gaudy Commodore [Internet]. 2007 [cited 2019 Nov 10]. Available from: https://commons.wikimedia.org/wiki/File:Precis_octavia-01_(xndr).jpg

470. M P. Precis octavia from inland of Port Shepstone, KwaZulu-Natal [Internet]. 2010 [cited 2019 Nov 15]. Available from: https://commons.wikimedia.org/wiki/File:Gaudy_Commodore_winter_form.JPG

471. Huët J. Ambystoma mavortium [Internet]. 1866 [cited 2019 Nov 10]. Available from: https://commons.wikimedia.org/wiki/File:Ambystoma_mavortium.jpg

472. Ribeiro CI. Ambystoma tigrinum [Internet]. 2009 [cited 2019 Nov 10]. Available from: https://commons.wikimedia.org/wiki/File:Salamandra_Tigre.png

473. Whiteman HH. Evolution of Facultative Paedomorphosis in Salamanders. The Quarterly Review of Biology. 1994;69(2):205-21. doi:10.1086/418540

474. Chmee2. Lakušník vodní (Batrachium aquatile/Ranunculus aquatilis). [Internet]. 2011 [cited 2019 Nov 15]. Available from:

https://commons.wikimedia.org/wiki/File:Ranunculus_aquatilis_in_Vlkovska_piskovna_(6).J PG

475. Otto Wilhelm T. Ranunculus aquatilis [Internet]. [cited 2019 Nov 10]. Available from: https://commons.wikimedia.org/wiki/File:Illustration_Ranunculus_aquatilis1.jpg

476. https://unsplash.com/@r3dmax JP. Polarfuchs (Vulpes lagopus) mit Winterfell im Schnee, Island [Internet]. 2017 [cited 2019 Nov 10]. Available from:

https://commons.wikimedia.org/wiki/File:Iceland-1979445.jpg

477. Service BM US Fish and Wildlife. Fox pups animals.jpg [Internet]. 2013 [cited 2019 Nov 15]. Available from: https://commons.wikimedia.org/wiki/File:Fox_pups_animals.jpg

478. Norén K, Dalén L, Flagstad Ø, Berteaux D, Wallén J, Angerbjörn A. Evolution, ecology and conservation-revisiting three decades of Arctic fox population genetic research. Polar Research. 2017 Sep 8;36(sup1):4. doi:10.1080/17518369.2017.1325135

479. Valena S, Moczek AP. Epigenetic Mechanisms Underlying Developmental Plasticity in Horned Beetles. Genetics Research International. 2012;2012:1-14. doi:10.1155/2012/576303

480. Hobgood N. Thalassoma bifasciatum (Bluehead Wrasse) juvenile yellow stage over Bispira brunnea (Social Feather Duster Worms). [Internet]. 2010 [cited 2019 Nov 15]. Available from:

https://commons.wikimedia.org/wiki/File:Thalassoma_bifasciatum_(Bluehead_Wrasse)_juve nile_yellow_stage_over_Bispira_brunnea_(Social_Feather_Duster_Worms).jpg 
481. Marcinek T. Bluehead Wrasse, Belize Barrier Reef [Internet]. 2007 [cited 2019 Nov 10]. Available from: https://commons.wikimedia.org/wiki/File:Bluhead_Wrasse.jpg

482. Warner RR, Swearer SE. Social Control of Sex Change in the Bluehead Wrasse, Thalassoma bifasciatum (Pisces: Labridae). The Biological Bulletin. 1991 Oct;181(2):199-204. doi:10.2307/1542090

483. Meloni M. The social brain meets the reactive genome: neuroscience, epigenetics and the new social biology. Front Hum Neurosci [Internet]. 2014 May 21 [cited 2019 Nov 18];8. doi:10.3389/fnhum.2014.00309

484. Berra TM. Freshwater Fish Distribution. [Internet]. Chicago: The University of Chicago Press; 2008 [cited 2019 Sep 3]. Available from: http://www.SLQ.eblib.com.au/patron/FullRecord.aspx?p=408286

485. Laudet V. The Origins and Evolution of Vertebrate Metamorphosis. Current Biology. 2011 Sep;21(18):R726-37. doi:10.1016/j.cub.2011.07.030

486. Waddington CH. Biological development. In: Encyclopedia Britannica [Internet]. Encyclopædia Britannica, inc.; 2019 [cited 2019 Sep 16]. Available from: https://www.britannica.com/science/biological-development

487. Gomez-Mestre I, Kulkarni S, Buchholz DR. Mechanisms and Consequences of Developmental Acceleration in Tadpoles Responding to Pond Drying. Navas CA, editor. PLoS ONE. 2013 Dec 16;8(12):e84266. doi:10.1371/journal.pone.0084266

488. Denver RJ, Crespi EJ. Stress Hormones and Human Developmental Plasticity: Lessons From Tadpoles. NeoReviews. 2006 Apr 1;7(4):e183-8. doi:10.1542/neo.7-4-e183

489. the Born Too Soon Preterm Birth Action Group, Howson CP, Kinney MV, McDougall L, Lawn JE. Born Too Soon: Preterm birth matters. Reprod Health. 2013 Nov;10(S1):S1. doi:10.1186/1742-4755-10-S1-S1

490. Althabe F, Howson CP, Kinney M, Lawn J, World Health Organization. Born too soon: the global action report on preterm birth [Internet]. 2012 [cited 2019 Sep 21]. Available from: http://www.who.int/pmnch/media/news/2012/201204\%5Fborntoosoon-report.pdf

491. Pregnancy Care Clinical Guidelines: Risk of Preterm Birth [Internet]. Australian Government Department of Health; 2019 [cited 2019 Sep 21]. Available from:

https://www.health.gov.au/resources/pregnancy-care-guidelines/part-d-clinical-assessments/ risk-of-preterm-birth

492. Wadhwa PD, Entringer S, Buss C, Lu MC. The Contribution of Maternal Stress to Preterm Birth: Issues and Considerations. Clinics in Perinatology. 2011 Sep;38(3):351-84. doi:10.1016/j.clp.2011.06.007

493. Mendelson CR, Montalbano AP, Gao L. Fetal-to-maternal signaling in the timing of birth. The Journal of Steroid Biochemistry and Molecular Biology. 2017 Jun;170:19-27. doi:10.1016/j.jsbmb.2016.09.006

494. Fernando F, Keijser R, Henneman P, van der Kevie-Kersemaekers A-MF, Mannens MM, van der Post JA, et al. The idiopathic preterm delivery methylation profile in umbilical cord blood DNA. BMC Genomics. 2015 Sep 29;16(1):736. doi:10.1186/s12864-015-1915-4 
495. Goldenberg RL, Culhane JF, Iams JD, Romero R. Epidemiology and causes of preterm birth. The Lancet. 2008 Jan;371(9606):75-84. doi:10.1016/S0140-6736(08)60074-4

496. Goffinet F. Primary predictors of preterm labour. BJOG: An International Journal of Obstetrics \& Gynaecology. 2005 Mar;112:38-47. doi:10.1111/j.1471-0528.2005.00583.x

497. Urquia ML, Glazier RH, Blondel B, Zeitlin J, Gissler M, Macfarlane A, et al. International migration and adverse birth outcomes: role of ethnicity, region of origin and destination. Journal of Epidemiology \& Community Health. 2010 Mar 1;64(3):243-51. doi:10.1136/jech.2008.083535

498. Newnham JP, Dickinson JE, Hart RJ, Pennell CE, Arrese CA, Keelan JA. Strategies to Prevent Preterm Birth. Front Immunol [Internet]. 2014 Nov 19 [cited 2019 Sep 29];5. doi:10.3389/fimmu.2014.00584

499. Pember MA. The Midwives' Resistance: How Native Women Are Reclaiming Birth on Their Terms. RewireNews [Internet]. 2018 Jan 5 [cited 2019 Sep 29]; Available from: https://rewire.news/article/2018/01/05/midwives-resistance-native-women-reclaiming-birthterms/

500. Shah PS, Zao J, Al-Wassia H, Shah V. Pregnancy and Neonatal Outcomes of Aboriginal Women: A Systematic Review and Meta-Analysis. Women's Health Issues. 2011 Jan;21(1):28-39. doi:10.1016/j.whi.2010.08.005

501. Kildea S, Gao Y, Hickey S, Kruske S, Nelson C, Blackman R, et al. Reducing preterm birth amongst Aboriginal and Torres Strait Islander babies: A prospective cohort study, Brisbane, Australia. EClinicalMedicine. 2019 Jul;12:43-51. doi:10.1016/j.eclinm.2019.06.001

502. Sandman CA, Davis EP, Glynn LM. Prescient Human Fetuses Thrive. Psychol Sci. 2012 Jan;23(1):93-100. doi:10.1177/0956797611422073

503. Steroid Endocrinology of Pregnancy. GLOWM [Internet]. 2009 [cited 2019 Oct 2]; doi:10.3843/GLOWM.10311

504. Binder MD, Hirokawa N, Windhorst U, editors. Neuroendocrine Axis. In: Encyclopedia of Neuroscience [Internet]. Berlin, Heidelberg: Springer Berlin Heidelberg; 2009 [cited 2019 Oct 11]. p. 2624-2624. doi:10.1007/978-3-540-29678-2_3823

505. Petraglia F, editor. Preterm birth: mechanisms, mediators, prediction, prevention and interventions. Abingdon: Informa Healthcare; 2007. 204 p.

506. Denver RJ. Structural and Functional Evolution of Vertebrate Neuroendocrine Stress Systems. Annals of the New York Academy of Sciences. 2009 Apr;1163(1):1-16. doi:10.1111/j.1749-6632.2009.04433.x

507. Denver RJ. Environmental Stress as a Developmental Cue: Corticotropin-Releasing Hormone Is a Proximate Mediator of Adaptive Phenotypic Plasticity in Amphibian Metamorphosis. Hormones and Behavior. 1997 Apr;31(2):169-79. doi:10.1006/hbeh.1997.1383

508. Schulkin J. Evolutionary conservation of glucocorticoids and corticotropin releasing hormone: Behavioral and physiological adaptations. Brain Research. 2011 May;1392:27-46. doi:10.1016/j.brainres.2011.03.055 
509. Wadhwa PD, Garite TJ, Porto M, Glynn L, Chicz-DeMet A, Dunkel-Schetter C, et al. Placental corticotropin-releasing hormone (CRH), spontaneous preterm birth, and fetal growth restriction: A prospective investigation. American Journal of Obstetrics and Gynecology. 2004 Oct;191(4):1063-9. doi:10.1016/j.ajog.2004.06.070

510. Sandman CA, Glynn LM. Corticotropin-releasing hormone programs the fetal and maternal brain. Future Neurology. 2009 May;4(3):257-61. doi:10.2217/fnl.09.8

511. Sandman CA, Davis EP, Buss C, Glynn LM. Prenatal Programming of Human Neurological Function. International Journal of Peptides. 2011;2011:1-9. doi:10.1155/2011/837596

512. Findings from the Global Burden of Disease Study 2017. Seattle, WA: Institute for Health Metrics and Evaluation (IHME).; 2018.

513. Pearce N, Ebrahim S, McKee M, Lamptey P, Barreto ML, Matheson D, et al. Global prevention and control of NCDs: Limitations of the standard approach. J Public Health Pol. 2015 Nov;36(4):408-25. doi:10.1057/jphp.2015.29

514. Hunter DJ, Reddy KS. Noncommunicable Diseases. N Engl J Med. 2013 Oct 3;369(14):1336-43. doi:10.1056/NEJMra1109345

515. WHO Fact Sheet: Noncommunicable diseases [Internet]. 2018 [cited 2019 Oct 22]. Available from: https://www.who.int/en/news-room/fact-sheets/detail/noncommunicable-diseases

516. Kirch W, editor. Encyclopedia of public health. Dordrecht: Springer; 2008.

517. Islam SMS, Purnat TD, Phuong NTA, Mwingira U, Schacht K, Fröschl G. NonCommunicable Diseases (NCDs) in developing countries: a symposium report. Global Health. 2014 Dec;10(1):81. doi:10.1186/s12992-014-0081-9

518. Bennett JE, Stevens GA, Mathers CD, Bonita R, Rehm J, Kruk ME, et al. NCD Countdown 2030: worldwide trends in non-communicable disease mortality and progress towards Sustainable Development Goal target 3.4. The Lancet. 2018 Sep;392(10152):1072-88. doi:10.1016/S0140-6736(18)31992-5

519. Hanson MA, Gluckman PD. Early Developmental Conditioning of Later Health and Disease: Physiology or Pathophysiology? Physiological Reviews. 2014 Oct;94(4):1027-76. doi:10.1152/physrev.00029.2013

520. Bloom D, Cafiero E, Jané-Llopis E, Abrahams-Gessel S, Bloom L, Fathima S, et al. The Global Economic Burden of Noncommunicable Diseases [Internet]. Program on the Global Demography of Aging; 2012 Jan. Available from: https://EconPapers.repec.org/RePEc:gdm:wpaper:8712

521. Manolio TA, Collins FS, Cox NJ, Goldstein DB, Hindorff LA, Hunter DJ, et al. Finding the missing heritability of complex diseases. Nature. 2009 Oct;461(7265):747-53. doi:10.1038/nature08494

522. Kogan MD. Social causes of low birth weight. J R Soc Med. 1995 Nov;88(11):611-5.

523. Sweatt JD. The Emerging Field of Neuroepigenetics. Neuron. 2013 Oct;80(3):624-32. doi:10.1016/j.neuron.2013.10.023 
524. Szyf M, Bick J. DNA Methylation: A Mechanism for Embedding Early Life Experiences in the Genome. Child Development. 2013 Jan;84(1):49-57. doi:10.1111/j.1467-

8624.2012.01793.x

525. Meaney FJ, Taylor C. Heritability. In: Encyclopedia Britannica [Internet]. Encyclopædia Britannica, inc.; 2018 [cited 2020 Jan 24]. Available from:

https://www.britannica.com/science/heritability

526. Trerotola M, Relli V, Simeone P, Alberti S. Epigenetic inheritance and the missing heritability. Hum Genomics. 2015 Dec;9(1):17. doi:10.1186/s40246-015-0041-3

527. Lynch K. Explainer: what is heritability? [Internet]. The Conversation. [cited 2020 Jan 24]. Available from: http://theconversation.com/explainer-what-is-heritability-21334

528. Plomin R, Deary IJ. Genetics and intelligence differences: five special findings. Mol Psychiatry. 2015 Feb;20(1):98-108. doi:10.1038/mp.2014.105

529. Savage JE, Jansen PR, Stringer S, Watanabe K, Bryois J, de Leeuw CA, et al. Genome-wide association meta-analysis in 269,867 individuals identifies new genetic and functional links to intelligence. Nat Genet. 2018 Jul;50(7):912-9. doi:10.1038/s41588-018-0152-6

530. the IMAGEN consortium, Kaminski JA, Schlagenhauf F, Rapp M, Awasthi S, Ruggeri B, et al. Epigenetic variance in dopamine D2 receptor: a marker of IQ malleability? Transl Psychiatry. 2018 Dec;8(1):169. doi:10.1038/s41398-018-0222-7

531. Hill WD, Marioni RE, Maghzian O, Ritchie SJ, Hagenaars SP, McIntosh AM, et al. A combined analysis of genetically correlated traits identifies 187 loci and a role for neurogenesis and myelination in intelligence. Mol Psychiatry. 2019 Feb;24(2):169-81. doi:10.1038/s41380-017-0001-5

532. Murrell A, Rakyan VK, Beck S. From genome to epigenome. Human Molecular Genetics. 2005 Apr 15;14(suppl_1):R3-10. doi:10.1093/hmg/ddi110

533. Thiagarajan RD, Morey R, Laurent LC. The epigenome in pluripotency and differentiation. Epigenomics. 2014 Feb;6(1):121-37. doi:10.2217/epi.13.80

534. Baverstock K, Rönkkö M. Epigenetic Regulation of the Mammalian Cell. Imhof A, editor. PLoS ONE. 2008 Jun 4;3(6):e2290. doi:10.1371/journal.pone.0002290

535. Weinhold B. Epigenetics: The Science of Change. Environmental Health Perspectives [Internet]. 2006 Mar [cited 2019 Dec 21];114(3). doi:10.1289/ehp.114-a160

536. OpenStax. DNA Macrostructure [Internet]. 2016 [cited 2019 Dec 20]. Available from: https://commons.wikimedia.org/wiki/File:0321_DNA_Macrostructure.jpg

537. Ganesan A, Arimondo PB, Rots MG, Jeronimo C, Berdasco M. The timeline of epigenetic drug discovery: from reality to dreams. Clin Epigenet. 2019 Dec;11(1):174. doi:10.1186/s13148-019-0776-0

538. Epigenetic mechanisms [Internet]. 2005 [cited 2019 Dec 18]. Available from: https://commons.wikimedia.org/wiki/File:Epigenetic_mechanisms.jpg 
539. Pacchierotti F, Spanò M. Environmental Impact on DNA Methylation in the Germline: State of the Art and Gaps of Knowledge. BioMed Research International. 2015;2015:1-23. doi:10.1155/2015/123484

540. Kelly TK, De Carvalho DD, Jones PA. Epigenetic modifications as therapeutic targets. Nat Biotechnol. 2010 Oct;28(10):1069-78. doi:10.1038/nbt.1678

541. Wu J, Brown M. Epigenetics and Epigenomics. In: Hematology [Internet]. 7th ed. Elsevier; 2018 [cited 2020 Feb 3]. p. 17-24. doi:10.1016/B978-0-323-35762-3.00002-0

542. Wadhwa PD, Glynn L, Hobel CJ, Garite TJ, Porto M, Chicz-DeMet A, et al. Behavioral perinatology: Biobehavioral processes in human fetal development. Regulatory Peptides. 2002 Oct;108(2-3):149-57. doi:10.1016/S0167-0115(02)00102-7

543. McGowan PO, Suderman M, Sasaki A, Huang TCT, Hallett M, Meaney MJ, et al. Broad Epigenetic Signature of Maternal Care in the Brain of Adult Rats. Sirigu A, editor. PLoS ONE. 2011 Feb 28;6(2):e14739. doi:10.1371/journal.pone.0014739

544. Wolf JB, Wade MJ. What are maternal effects (and what are they not)? Phil Trans R Soc B. 2009 Apr 27;364(1520):1107-15. doi:10.1098/rstb.2008.0238

545. Hunt J, Simmons LW. Optimal maternal investment in the dung beetle Onthophagus taurus? Behavioral Ecology and Sociobiology. 2004 Jan 1;55(3):302-12. doi:10.1007/s00265-0030705-1

546. Mommer BC, Bell AM. A test of maternal programming of offspring stress response to predation risk in threespine sticklebacks. Physiology \& Behavior. 2013 Oct;122:222-7. doi:10.1016/j.physbeh.2013.04.004

547. Ducatez S, Baguette M, Stevens VM, Legrand D, Fréville H. Complex Interactions between Paternal and Maternal Effects: Parental Experience and Age at Reproduction Affect Fecundity and Offspring Performance in a Butterfly. Evolution. 2012 Nov;66(11):3558-69. doi:10.1111/j.1558-5646.2012.01704.x

548. Curley JP, Mashoodh R, Champagne FA. Epigenetics and the origins of paternal effects. Hormones and Behavior. 2011 Mar;59(3):306-14. doi:10.1016/j.yhbeh.2010.06.018

549. Yao Y, Robinson AM, Zucchi FC, Robbins JC, Babenko O, Kovalchuk O, et al. Ancestral exposure to stress epigenetically programs preterm birth risk and adverse maternal and newborn outcomes. BMC Med. 2014 Dec;12(1):121. doi:10.1186/s12916-014-0121-6

550. Meaney MJ, Szyf M. Environmental programming of stress responses through DNA methylation: life at the interface between a dynamic environment and a fixed genome. Dialogues Clin Neurosci. 2005;7(2):103-23.

551. Kapoor A, Dunn E, Kostaki A, Andrews MH, Matthews SG. Fetal programming of hypothalamo-pituitary-adrenal function: prenatal stress and glucocorticoids: Fetal programming of HPA function. The Journal of Physiology. 2006 Apr;572(1):31-44. doi:10.1113/jphysiol.2006.105254

552. Champagne FA, Francis DD, Mar A, Meaney MJ. Variations in maternal care in the rat as a mediating influence for the effects of environment on development. Physiology \& Behavior. 2003 Aug;79(3):359-71. doi:10.1016/S0031-9384(03)00149-5 
553. Francis D. Nongenomic Transmission Across Generations of Maternal Behavior and Stress Responses in the Rat. Science. 1999 Nov 5;286(5442):1155-8.

doi:10.1126/science.286.5442.1155

554. Guzzetta A, Baldini S, Bancale A, Baroncelli L, Ciucci F, Ghirri P, et al. Massage Accelerates Brain Development and the Maturation of Visual Function. Journal of Neuroscience. 2009 May 6;29(18):6042-51. doi:10.1523/JNEUROSCI.5548-08.2009

555. Kaffman A, Meaney MJ. Neurodevelopmental sequelae of postnatal maternal care in rodents: clinical and research implications of molecular insights. J Child Psychol \& Psychiat. 2007 Mar;48(3-4):224-44. doi:10.1111/j.1469-7610.2007.01730.x

556. Leon M. Development of Thermoregulation. In: Blass EM, editor. Developmental Psychobiology and Developmental Neurobiology [Internet]. Boston, MA: Springer US; 1986 [cited 2019 Nov 23]. p. 297-322. doi:10.1007/978-1-4613-2113-2_8

557. Paré WP, Glavin GB. Animal models of stress in pharmacology. In: Techniques in the Behavioral and Neural Sciences [Internet]. Elsevier; 1993 [cited 2019 Nov 23]. p. 413-41. doi:10.1016/B978-0-444-81444-9.50021-3

558. Brouette-Lahlou I, Vernet-Maury E, Chastrette F, Chanel J. Maternal Anogenital Licking in Rats: Exploring the Dam’s Differential Sexual Treatment of Pups. In: Marchlewska-Koj A, Lepri JJ, Müller-Schwarze D, editors. Chemical Signals in Vertebrates 9 [Internet]. Boston, MA: Springer US; 2001 [cited 2019 Nov 23]. p. 269-75. doi:10.1007/978-1-4615-0671-3_36

559. Turecki G, Meaney MJ. Effects of the Social Environment and Stress on Glucocorticoid Receptor Gene Methylation: A Systematic Review. Biological Psychiatry. 2016 Jan;79(2):8796. doi:10.1016/j.biopsych.2014.11.022

560. Weaver ICG, Cervoni N, Champagne FA, D’Alessio AC, Sharma S, Seckl JR, et al. Epigenetic programming by maternal behavior. Nat Neurosci. 2004 Aug;7(8):847-54. doi:10.1038/nn1276

561. Caldji C, Tannenbaum B, Sharma S, Francis D, Plotsky PM, Meaney MJ. Maternal care during infancy regulates the development of neural systems mediating the expression of fearfulness in the rat. Proceedings of the National Academy of Sciences. $1998 \mathrm{Apr}$ 28;95(9):5335-40. doi:10.1073/pnas.95.9.5335

562. Siviy SM, Harrison KA. Effects of neonatal handling on play behavior and fear towards a predator odor in juvenile rats (Rattus norvegicus). Journal of Comparative Psychology. 2008;122(1):1-8. doi:10.1037/0735-7036.122.1.1

563. Francis DD, Kuhar MJ. Frequency of maternal licking and grooming correlates negatively with vulnerability to cocaine and alcohol use in rats. Pharmacology Biochemistry and Behavior. 2008 Sep;90(3):497-500. doi:10.1016/j.pbb.2008.04.012

564. Szyf M. How early life experience is written into DNA [Internet]. [cited 2019 Nov 28]. Available from: https://www.ted.com/talks/moshe_szyf_how_early_life_experience_is_written_into_dna

565. Provencal N, Suderman MJ, Guillemin C, Massart R, Ruggiero A, Wang D, et al. The Signature of Maternal Rearing in the Methylome in Rhesus Macaque Prefrontal Cortex and T 
Cells. Journal of Neuroscience. 2012 Oct 31;32(44):15626-42.

doi:10.1523/JNEUROSCI.1470-12.2012

566. Jirtle RL. The Agouti mouse: a biosensor for environmental epigenomics studies investigating the developmental origins of health and disease. Epigenomics. 2014 Oct;6(5):447-50. doi:10.2217/epi.14.58

567. Dolinoy RJ and D. Agouti viable yellow (Avy) mice [Internet]. 2007 [cited 2019 Dec 7]. Available from: https://commons.wikimedia.org/wiki/File:Agouti_Mice.jpg

568. Morgan HD, Sutherland HGE, Martin DIK, Whitelaw E. Epigenetic inheritance at the agouti locus in the mouse. Nat Genet. 1999 Nov;23(3):314-8. doi:10.1038/15490

569. Agouti (gene). In: Wikipedia [Internet]. 2019 [cited 2019 Dec 9]. Available from: https://en.wikipedia.org/w/index.php?title=Agouti_(gene)\&oldid=929288363

570. Dolinoy DC. The agouti mouse model: an epigenetic biosensor for nutritional and environmental alterations on the fetal epigenome. Nutrition Reviews. 2008 Jul 29;66(No. s1):S7-11. doi:10.1111/j.1753-4887.2008.00056.x

571. Anda RF, Butchart A, Felitti VJ, Brown DW. Building a Framework for Global Surveillance of the Public Health Implications of Adverse Childhood Experiences. American Journal of Preventive Medicine. 2010 Jul;39(1):93-8. doi:10.1016/j.amepre.2010.03.015

572. Cao-Lei L, Massart R, Suderman MJ, Machnes Z, Elgbeili G, Laplante DP, et al. DNA Methylation Signatures Triggered by Prenatal Maternal Stress Exposure to a Natural Disaster: Project Ice Storm. Iwamoto K, editor. PLoS ONE. 2014 Sep 19;9(9):e107653. doi:10.1371/journal.pone.0107653

573. Sun G, Fu L, Shi Y-B. Epigenetic regulation of thyroid hormone-induced adult intestinal stem cell development during anuran metamorphosis. Cell Biosci. 2014;4(1):73.

doi:10.1186/2045-3701-4-73

574. Keller H, Bard KA, editors. The cultural nature of attachment: contextualizing relationships and development. Cambridge, Massachusetts: The MIT Press; 2017. 429 p. (Strungmann forum reports).

575. Jablonka E, Lamb MJ, Zeligowski A. Evolution in four dimensions: genetic, epigenetic, behavioral, and symbolic variation in the history of life. Revised edition. Cambridge, Massachusetts ; London, England: A Bradford Book, The MIT Press; 2014. 563 p. (Life and mind: philosophical issues in biology and psychology).

576. Yaden DB, Iwry J, Slack KJ, Eichstaedt JC, Zhao Y, Vaillant GE, et al. The overview effect: Awe and self-transcendent experience in space flight. Psychology of Consciousness: Theory, Research, and Practice. 2016;3(1):1-11. doi:10.1037/cns0000086

577. Johnstone B, Cohen D. The Nature of Spiritual Transcendence. In: Neuroscience, Selflessness, and Spiritual Experience [Internet]. Elsevier; 2019 [cited 2020 Mar 19]. p. 1940. doi:10.1016/B978-0-08-102218-4.00002-X

578. Garcia-Romeu A, Himelstein SP, Kaminker J. Self-transcendent experience: a grounded theory study. Qualitative Research. 2015 Oct;15(5):633-54. doi:10.1177/1468794114550679 
579. Kastrup B. Self-Transcendence Correlates with Brain Function Impairment. Journal of Cognition and Neuroethics. 2017;4(3):33-42.

580. Mancini AD. When acute adversity improves psychological health: A social-contextual framework. Psychological Review. 2019 Jul;126(4):486-505. doi:10.1037/rev0000144

581. Ramos C, Leal I. Posttraumatic Growth in the Aftermath of Trauma: A Literature Review About Related Factors and Application Contexts. PCH. 2013 Mar 28;2(1):43-54. doi:10.5964/pch.v2i1.39

582. Jayawickreme E, Blackie LER. Exploring the Psychological Benefits of Hardship: a Critical Reassessment of Posttraumatic Growth [Internet]. 2016 [cited 2020 Mar 29]. Available from: https://doi.org/10.1007/978-3-319-47989-7

583. Jayawickreme E, Blackie LER. Post-traumatic Growth as Positive Personality Change: Evidence, Controversies and Future Directions. Eur J Pers. 2014 Jul;28(4):312-31. doi:10.1002/per.1963

584. Sugiura M, Sato S, Nouchi R, Honda A, Abe T, Muramoto T, et al. Eight Personal Characteristics Associated with the Power to Live with Disasters as Indicated by Survivors of the 2011 Great East Japan Earthquake Disaster. Qiu J, editor. PLoS ONE. 2015 Jul 1;10(7):e0130349. doi:10.1371/journal.pone.0130349

585. Sugiura M. The Power to Live with Disasters: Adaptive Believing Processes of the Self and World. In: Angel H-F, Oviedo L, Paloutzian RF, Runehov ALC, Seitz RJ, editors. Processes of Believing: The Acquisition, Maintenance, and Change in Creditions [Internet]. Cham: Springer International Publishing; 2017 [cited 2020 Mar 20]. p. 139-52. (New Approaches to the Scientific Study of Religion; vol. 1). doi:10.1007/978-3-319-50924-2_10

586. Maslow AH. The farther reaches of human nature. New York, N.Y., U.S.A: Arkana; 1993. $407 \mathrm{p}$.

587. Big Five personality traits. In: Wikipedia [Internet]. 2020 [cited 2020 Mar 31]. Available from: https://en.wikipedia.org/w/index.php? title=Big_Five_personality_traits\&oldid=948158213

588. Martin P, Kelly N, Kahana B, Kahana E, Willcox BJ, Willcox DC, et al. Defining Successful Aging: A Tangible or Elusive Concept? The Gerontologist. 2015 Feb 1;55(1):14-25. doi:10.1093/geront/gnu044

589. Tornstam L. Gerotranscendence: a developmental theory of positive aging. New York: Springer Pub. Co; 2005. 213 p.

590. Levenson MR, Jennings PA, Aldwin CM, Shiraishi RW. Self-Transcendence: Conceptualization and Measurement. Int J Aging Hum Dev. 2005 Mar;60(2):127-43. doi:10.2190/XRXM-FYRA-7U0X-GRC0

591. Aldwin CM, Igarashi H, Gilmer DF, Levenson MR. Health, illness, and optimal aging: biological and psychosocial perspectives. Third edition. New York, NY: Springer Publishing Company; 2018. 414 p.

592. Rowe JW, Kahn RL. Successful Aging 2.0: Conceptual Expansions for the 21st Century. GERONB. 2015 Jul;70(4):593-6. doi:10.1093/geronb/gbv025 
593. Stowe JD, Cooney TM. Examining Rowe and Kahn's Concept of Successful Aging: Importance of Taking a Life Course Perspective. The Gerontologist. 2015 Feb 1;55(1):43-50. doi:10.1093/geront/gnu055

594. Montz BE, Tobin GA, Hagelman RR, Tobin GA. Natural hazards: explanation and integration. Second Edition. New York: The Guilford Press; 2017. 445 p.

595. Ritchie H, Roser M. Natural Disasters. In: Our World in Data [Internet]. 2020. Available from: https://ourworldindata.org/natural-disasters

596. Disasters and FEMA aid Exacerbate Racial Inequality [Internet]. [cited 2020 Apr 17]. Available from: https://www.youtube.com/watch?v=j3YiXWYxI3U\&feature=emb_logo

597. Howell J, Elliott JR. Damages Done: The Longitudinal Impacts of Natural Hazards on Wealth Inequality in the United States. Social Problems. 2019 Aug 1;66(3):448-67. doi:10.1093/socpro/spy016

598. Davies IP, Haugo RD, Robertson JC, Levin PS. The unequal vulnerability of communities of color to wildfire. Jones JA, editor. PLoS ONE. 2018 Nov 2;13(11):e0205825. doi:10.1371/journal.pone.0205825

599. Ratcliffe C, Congdon WJ, Stanczyk A, Teles D, Martín C, Kotapati B. Insult to Injury: Natural Disasters and Residents’ Financial Health. Washington, DC: Urban Institute; 2019 p. 72.

600. Bowman DMJS, Balch JK, Artaxo P, Bond WJ, Carlson JM, Cochrane MA, et al. Fire in the Earth System. Science. 2009 Apr 24;324(5926):481-4. doi:10.1126/science.1163886

601. Doerr SH, Santín C. Global trends in wildfire and its impacts: perceptions versus realities in a changing world. Phil Trans R Soc B. 2016 Jun 5;371(1696):20150345. doi:10.1098/rstb.2015.0345

602. He T, Lamont BB, Pausas JG. Fire as a key driver of Earth’s biodiversity. Biol Rev. 2019 Dec;94(6):1983-2010. doi:10.1111/brv.12544

603. Pausas JG, Keeley JE. Wildfires as an ecosystem service. Front Ecol Environ. 2019 Jun;17(5):289-95. doi:10.1002/fee.2044

604. Sober E. Evolution, Population Thinking, and Essentialism. Philosophy of Science. 1980 Sep;47(3):350-83. doi:10.1086/288942

605. Sober E. Philosophy of biology. 2nd ed. Boulder, Colo: Westview Press; 2000. 236 p. (Dimensions of philosophy series).

606. Offnfopt. Simple Periodic Table Chart [Internet]. 2017 [cited 2020 Mar 3]. Available from: https://commons.wikimedia.org/wiki/File:Simple_Periodic_Table_Chart-en.svg

607. Urry J. The Complexity Turn. Theory, Culture \& Society. 2005 Oct;22(5):1-14. doi:10.1177/0263276405057188

608. Maslin MA, Brierley CM, Milner AM, Shultz S, Trauth MH, Wilson KE. East African climate pulses and early human evolution. Quaternary Science Reviews. 2014 Oct;101:1-17. doi:10.1016/j.quascirev.2014.06.012 
609. Maslin MA, Christensen B. Tectonics, orbital forcing, global climate change, and human evolution in Africa: introduction to the African paleoclimate special volume. Journal of Human Evolution. 2007 Nov;53(5):443-64. doi:10.1016/j.jhevol.2007.06.005

610. https://www.uctv.tv U University of California Television-. VIDEO: CARTA: HumanClimate Interactions and Evolution: Past and Future: Rick Potts: Climate Instability and the Evolution of Human Adaptability. [Internet]. [cited 2020 Feb 20]. Available from: https://www.uctv.tv/shows/CARTA-Human-Climate-Interactions-and-Evolution-Past-andFuture-Rick-Potts-Climate-Instability-and-the-Evolution-of-Human-Adaptability-29690

611. Maslin MA, Shultz S, Trauth MH. A synthesis of the theories and concepts of early human evolution. Phil Trans R Soc B. 2015 Mar 5;370(1663):20140064. doi:10.1098/rstb.2014.0064

612. Magill CR, Ashley GM, Freeman KH. Ecosystem variability and early human habitats in eastern Africa. Proc Natl Acad Sci USA. 2013 Jan 22;110(4):1167-74. doi:10.1073/pnas.1206276110

613. Potts R, Faith JT. Alternating high and low climate variability: The context of natural selection and speciation in Plio-Pleistocene hominin evolution. Journal of Human Evolution. 2015 Oct;87:5-20. doi:10.1016/j.jhevol.2015.06.014

614. Climate Effects on Human Evolution [Internet]. The Smithsonian Institution's Human Origins Program. 2010 [cited 2020 Feb 22]. Available from:

http://humanorigins.si.edu/research/climate-and-human-evolution/climate-effects-humanevolution

615. Roser M. Mortality in the past - around half died as children. In: Our World in Data [Internet]. 2019 [cited 2020 Feb 24]. Available from: https://ourworldindata.org/childmortality-in-the-past

616. Burghardt GM. Play in fishes, frogs and reptiles. Current Biology. 2015 Jan;25(1):R9-10. doi:10.1016/j.cub.2014.10.027

617. Burghardt GM. The genesis of animal play: testing the limits. Cambridge, Mass: MIT Press; 2005. 501 p.

618. Vaughan C, Brown S. Play: how it shapes the brain, opens the imagination, and invigorates the soul [Internet]. New York: Avery; 2014 [cited 2020 Mar 11]. Available from: http://rbdigital.oneclickdigital.com

619. Copeland WE, Shanahan L, Hinesley J, Chan RF, Aberg KA, Fairbank JA, et al. Association of Childhood Trauma Exposure With Adult Psychiatric Disorders and Functional Outcomes. JAMA Netw Open. 2018 Nov 9;1(7):e184493. doi:10.1001/jamanetworkopen.2018.4493

620. Gregorowski C, Seedat S. Addressing childhood trauma in a developmental context. Journal of Child \& Adolescent Mental Health. 2013 Dec;25(2):105-18.

doi:10.2989/17280583.2013.795154

621. De Bellis MD, Zisk A. The Biological Effects of Childhood Trauma. Child and Adolescent Psychiatric Clinics of North America. 2014 Apr;23(2):185-222.

doi:10.1016/j.chc.2014.01.002 
622. Birn A-E, Pillay Y, Holtz TH. Textbook of global health. Fourth edition. New York, NY: Oxford University Press; 2017. 674 p.

623. Countries Falling Behind In Meeting Noncommunicable Disease Control Targets - Health Policy Watch [Internet]. [cited 2020 Apr 22]. Available from: https://www.healthpolicywatch.org/countries-falling-behind-in-meeting-noncommunicable-disease-control-targets/

624. Glasgow S, Schrecker T. The double burden of neoliberalism? Noncommunicable disease policies and the global political economy of risk. Health \& Place. 2015 Jul;34:279-86. doi:10.1016/j.healthplace.2015.06.005

625. Kriznik NM, Kinmonth AL, Ling T, Kelly MP. Moving beyond individual choice in policies to reduce health inequalities: the integration of dynamic with individual explanations. Journal of Public Health. 2018 Dec 1;40(4):764-75. doi:10.1093/pubmed/fdy045

626. Branca F, Lartey A, Oenema S, Aguayo V, Stordalen GA, Richardson R, et al. Transforming the food system to fight non-communicable diseases. BMJ. 2019 Jan 28;1296.

doi:10.1136/bmj.1296

627. Borsboom D. A network theory of mental disorders. World Psychiatry. 2017 Feb;16(1):5-13. doi:10.1002/wps.20375

628. Stange KC. A science of connectedness. Annals of Family Medicine. 2009 Oct;7(5):387-95. doi:10.1370/afm.990

629. Roser M, Ortiz-Ospina E. World Population Growth. In: Our World in Data [Internet]. 2013 [cited 2019 Mar 15]. Available from: https://ourworldindata.org/world-population-growth

630. Healthy People - HP2010 - Leading Health Indicators at a Glance [Internet]. [cited 2019 Apr 16]. Available from:

https://www.cdc.gov/nchs/healthy_people/hp2010/hp2010_indicators.htm

631. urban sprawl | Description, Causes, Environmental Impacts, \& Alternatives | Britannica.com [Internet]. [cited 2019 Apr 16]. Available from: https://www.britannica.com/topic/urbansprawl

632. Romer CD, Pells RH. Great Depression | Definition, History, Causes, Effects, \& Facts. In: Encyclopedia Britannica [Internet]. Encyclopædia Britannica, inc.; 2019 [cited 2019 Apr 24]. Available from: https://www.britannica.com/event/Great-Depression

633. Frumkin H. Urban sprawl and public health. Public Health Reports. 2002 May;117(3):20117. doi:10.1016/S0033-3549(04)50155-3

634. European Americans. In: Wikipedia [Internet]. 2019 [cited 2019 Apr 22]. Available from: https://en.wikipedia.org/w/index.php?title=European_Americans\&oldid=893532847

635. Batur P, Feagin JR, editors. Handbook of the Sociology of Racial and Ethnic Relations. Second edition. Cham: Springer International Publishing; 2018. 434 p. (Handbooks of Sociology and Social Research).

636. UNICEF, International Child Development Centre. A league table of teenage births in rich nations. Florence, Italy: United Nations Children’s Fund, Innocenti Research Centre; 2001. 
637. The Myth of the Normal Brain: Embracing Neurodiversity. The AMA Journal of Ethics. 2015 Apr 1;17(4):348-52. doi:10.1001/journalofethics.2015.17.4.msoc1-1504

638. Des Roches S, Post DM, Turley NE, Bailey JK, Hendry AP, Kinnison MT, et al. The ecological importance of intraspecific variation. Nat Ecol Evol. 2018 Jan;2(1):57-64. doi:10.1038/s41559-017-0402-5

639. Fusco G, Minelli A. Phenotypic plasticity in development and evolution: facts and concepts. Introduction. Philos Trans R Soc Lond, B, Biol Sci. 2010 Feb 27;365(1540):547-56. doi:10.1098/rstb.2009.0267

640. Piersma T, Gils JA van. The flexible phenotype: a body-centred integration of ecology, physiology, and behaviour. Oxford: Oxford University Press; 2011. 238 p.

641. Forsman A. Rethinking phenotypic plasticity and its consequences for individuals, populations and species. Heredity. 2014 Oct 8;115:276.

642. Hormann AM, vom Saal FS, Nagel SC, Stahlhut RW, Moyer CL, Ellersieck MR, et al. Holding Thermal Receipt Paper and Eating Food after Using Hand Sanitizer Results in High Serum Bioactive and Urine Total Levels of Bisphenol A (BPA). Carpenter DO, editor. PLoS ONE. 2014 Oct 22;9(10):e110509. doi:10.1371/journal.pone.0110509

643. University of Missouri-Columbia. Thermal paper cash register receipts account for high bisphenol A (BPA) levels in humans. [cited 2019 Dec 9]; Available from: https://medicalxpress.com/news/2014-10-thermal-paper-cash-register-receipts.html

644. Wolstenholme JT, Rissman EF, Connelly JJ. The role of Bisphenol A in shaping the brain, epigenome and behavior. Hormones and Behavior. 2011 Mar;59(3):296-305.

doi:10.1016/j.yhbeh.2010.10.001 